\title{
EATING INSECTS:
}

CONSUMER ACCEPTANCE OF A

CULTURALLY INAPPROPRIATE FOOD

Hui Shan Grace Tan 


\section{Thesis committee}

\section{Promotor}

Prof. Dr Hans C. M. van Trijp

Professor of Marketing and Consumer Behaviour Group

Wageningen University \& Research

\section{Co-promotors}

Dr Markus Stieger

Associate professor, Division of Human Nutrition

Wageningen University \& Research

Dr Arnout R. H. Fischer

Associate professor, Marketing and Consumer Behaviour Group

Wageningen University \& Research

\section{Other members}

Prof. Dr Martinus A. J. S. van Boekel, Wageningen University \& Research

Prof. Dr Wim Verbeke, Ghent University, Ghent, Belgium

Prof. Dr Cor van der Weele, Wageningen University \& Research

Dr Michael Bom Frost, University of Copenhagen and Nordic Food Lab, Copenhagen, Denmark

This research was conducted under the auspices of the Graduate School VLAG (Food Technology, Agrobiotechnology, Nutrition and Health Sciences). 


\section{EATING INSECTS: \\ CONSUMER ACCEPTANCE OF A \\ CULTURALLY INAPPROPRIATE FOOD}

Hui Shan Grace Tan

Thesis

submitted in fulfilment of the requirements for the degree of doctor at Wageningen University

by the authority of the Rector Magnificus

Prof. Dr A.P.J. Mol,

in the presence of the

Thesis Committee appointed by the Academic Board

to be defended in public

on Tuesday, $16^{\text {th }}$ of May 2017

at 1:30 p.m. in the Aula. 


\section{Hui Shan Grace Tan}

Eating insects: Consumer acceptance of a culturally inappropriate food 174 pages.

PhD thesis, Wageningen University, Wageningen, NL (2017)

With references, with summary in English

ISBN 978-94-6343-152-1

DOI: http://dx.doi.org/10.18174/410874 


\section{CONTENTS}

CHAPTER 1 General introduction

CHAPTER 2 Insects as food: Exploring cultural exposure and individual experience as determinants of acceptance

CHAPTER 3 Tasty but nasty? Exploring the role of sensory-liking and food appropriateness in the willingness to eat unusual novel foods like insects

CHAPTER 4 Why do unusual novel foods like insects lack sensory appeal? Investigating the underlying sensory perceptions

CHAPTER 5 The influence of product preparation, familiarity and individual traits on the consumer acceptance of insects as food

CHAPTER 6 How will better products improve the sensory-liking and willingness to buy insect-based foods? 



\section{CHAPTER I General INRRodUCTION}




\subsection{INTRODUCTION}

Rapidly increasing global food demand has led to pressing concerns about the sustainability of food production systems (Godfray et al., 2010; Steinfeld, Gerber, Wassenaar, Castel, \& de Haan, 2006). In particular, large scale production of animal-based proteins severely impacts the environment and requires more measures than just increasing the efficiency of food production (Garnett, 2011).

Reducing the consumption of conventional meat has therefore become increasingly important. This has led to efforts to introduce alternative protein sources that can be more sustainably produced (Boland et al., 2012; Tilman, Balzer, Hill, \& Befort, 2011). As part of this initiative, edible insects are recently being introduced in the West as a source of high quality protein that can be produced with lower environmental impact than traditional livestock (e.g. lower greenhouse gas emission, higher feed-conversion ratios) (van Huis et al., 2013). Yet even though a large variety of species are regarded as tasty food in various cultures around the world (Chakravorty, Ghosh, \& Meyer-Rochow, 2011; Hanboonsong, 2010; Luo, 1997), the vast majority of Western consumers remains resistant to eating insects-a culturally inappropriate food. Most do not regard insects as food for humans, and view entomophagy (i.e. the practice of eating insects) as strange and disgusting (Kellert, 1993; Looy, Dunkel, \& Wood, 2014; Yen, 2009).

The lack of consumer acceptance is one of the key barriers to realizing the benefits of insect consumption, not to mention other regulatory, safety and technological challenges (Halloran, Vantomme, Hanboonsong, \& Ekesi, 2015; Rumpold \& Schlüter, 2013; van Huis, 2013; Yen, 2009). To address how consumer acceptance of insects as food may be achieved in the West, this thesis examines the determinants of consumer acceptance of culturally inappropriate foods.

\subsubsection{Challenges of introducing insects as food to the West}

According to the Food and Agricultural Organization (van Huis et al., 2013), "the case needs to be made to consumers that eating insects is not only good for their health, it is good for the planet". They make a bold assumption that consumers can be educated into changing their diet. Such rational arguments of 'why we should eat insects' have so far proven ineffective at overturning negative perceptions (Deroy, Reade, \& Spence, 2015; Looy et al., 2014). Nevertheless, it has contributed to growing interest in entomophagy as people become increasingly aware that insects are good to eat. Western consumers appear to have gone from dismissing it entirely (de Boer, Schösler, \& Boersema, 2013; Vanhonacker, Van Loo, Gellynck, \& Verbeke, 2013) to being more willing to consider eating insects, in some form, in the future (Caparros Megido et al., 2014; Ruby, Rozin, \& Chan, 2015). Despite this promising development, this has yet to translate into real consumption, i.e. the actual adoption of insect-based foods into the Western diet, which is necessary for the environmental benefits to be realized. 
The need to change negative Western perceptions has been repeatedly emphasized (Deroy et al., 2015; Looy et al., 2014; Yen, 2009) but little has been done so far to study how this can be achieved. A growing number of empirical studies have identified the psychological factors that influence the general or initial willingness to eat insects amongst Western consumers (Baker, Shin, \& Kim, 2016; Hartmann, Shi, Giusto, \& Siegrist, 2015; Ruby et al., 2015; Verbeke, 2015). While these studies provide insights into current Western consumer perceptions of insects as food, there is a need to better understand what gives rise to these perceptions amongst Western consumers and how they can be changed through exposure. Until now, the cultural, taste and product dimensions remain relatively unexplored when investigating the consumer acceptance of novel foods. These aspects play critical roles in food choice and acceptance (Meiselman, 2003; Rozin, 2006; Shepherd, 2001), and will be investigated in this thesis.

\subsubsection{Consumer acceptance of a culturally inappropriate food}

Culturally inappropriate foods are defined as items that are not considered by a culture to be fit for consumption' (Rozin \& Fallon, 1987). When studied in the Western context, examples of such foods include insects, dog, bat, cat, blood, viscera, some fermented foods as well as other delicacies of both plant and animal origin that are generally not recognised to be edible. While these are good foods from the biological standpoint, clearly not all nutritious substances are considered to be appropriate to eat. Consumers tend to justify their rejections of culturally inappropriate foods in terms of animal associations and aversive textural properties (Martins \& Pliner, 2006). However, taking into account that foods that are delicious in one culture can be disgusting in another culture, it is likely that human food preferences and aversions are better explained by people's underlying perceptions than by the item's physical nature (Harris, 1985).

The question of how culturally inappropriate foods could gain acceptance has only become relevant with recent efforts to introduce insects into the Western diet. While a large amount of research has been done to understand the consumer acceptance of novel foods and technologies (e.g. functional, genetically modified and organic foods) (Bäckström, Pirttilä-Backman, \& Tuorila, 2004; Frewer et al., 2004; Siro, Kapolna, Kapolna, \& Lugasi, 2008; Tobler, Visschers, \& Siegrist, 2011), little is understood about the consumer acceptance of culturally inappropriate foods, where a strong disgust characterises the rejection. Coverage of such foods in popular books (e.g. Hopkins (2014); Menzel and d'Aluisio (1998)) and television programmes (e.g. 'Extreme gourmet', 'Bizarre foods', 'Fear factor') have drawn much attention from the public, but have made little positive impact on consumer perceptions. Although these sources help to raise awareness of the edibility of foods from other cultures, they mainly provide entertainment value by relying on elements of disgust, adventure, amusement, and disbelief regarding the consumption of culturally inappropriate foods. 
Disgust, which is defined as the 'revulsion at the prospect of oral incorporation of an offensive object' (Rozin \& Fallon, 1987), poses new challenges for consumer acceptance that are not faced by other novel foods. Most novel foods that enter the market are innovative combinations of familiar food ingredients (e.g. new flavoured drink) that are still compatible to the local range of foods, and more easy to accept than unknown foods (Aqueveque, 2015). Moreover, some culturally inappropriate foods could be rejected on the mere basis of their strangeness (Hopkins, 2014; Seaton \& Gardner, 1959). Sometimes their presence or previous contact with the food to be consumed could be sufficient to result in rejection (Rozin \& Fallon, 1987). This adds layers of complexity to understanding the acceptance of a culturally inappropriate food, as decisions to eat may be less dependent on the actual taste qualities of the food.

This thesis aims to gain a better understanding of how culture contributes to consumer perceptions of a potential food, and how the taste of the food and the way the product is prepared could play a role in acceptance. The following sections of this chapter discuss what is known about culture (section 1.2), taste (section 1.3) and the product (section 1.4) as determinants of acceptance of culturally inappropriate foods, and how it will be examined in this thesis (section 5) to provide new insights into the challenges involved in introducing insects into Western diets.

\subsection{CULTURE AS A DETERMINANT OF ACCEPTANCE}

The role of culture in the acceptance or rejection of insects as food has been repeatedly emphasized (Looy et al., 2014; Yen, 2009), but research investigating how culture specifically contributes to the observed differences in acceptance is currently lacking. Where insects are concerned, studies of Western consumers report reasons related to the physical appearance of the species and various negative associations (e.g. pests and disease) (Harris, 1985; Martins \& Pliner, 2006), and examine how various psychological factors (e.g. food neophobia, disgust sensitivity, risk perceptions) predict consumers' negativity towards eating insects (Baker et al., 2016; Hartmann et al., 2015; Ruby et al., 2015). Yet there is nothing inherent in the Western culture that precludes the acceptance of insects as food, as evidenced by the Western communities in the past and present that practice entomophagy (Bodenheimer, 1951). Consumers in cultures where insects are regularly consumed as part of the regular diet greatly enjoy the taste of insects (Hanboonsong, 2010; Ramos-Elorduy, 1997), and are also exposed to a richness of supply and consumption practices involving insects. These suggest that beyond psychological differences that are usually studied, other contextual factors relating to the cultural environment (e.g. availability, food norms) are likely to play an important role in the acceptance of insects as food. 


\subsubsection{The role of culture in food acceptance}

Culture can be defined as "the collective programming of the mind that distinguishes one group or category of people from another" (Hofstede \& McCrae, 2004), and is often regarded to be a major determinant of food choice and behaviours (Rozin, 1996; Sobal \& Bisogni, 2009). It is a complex entity that includes knowledge, beliefs, values and customs that are shaped by a variety of geographical, social, economic and political factors, thereby influencing how we eat and what we eat (Fieldhouse, 1996). To date, cross-cultural research on food acceptance often focus on quantifying the psychological differences in perceptions, preferences and motivations (Hartmann et al., 2015; Jaeger, Andani, Wakeling, \& MacFie, 1998; Prescott \& Bell, 1995; Prescott, Young, O'Neill, Yau, \& Stevens, 2002). Studies like these acknowledge the forces of culture on food behaviours but provide little explanation for why certain cultural foods come to be accepted in spite of their apparently aversive nature and properties. Understanding how culture contributes to a food's acceptance would enable more effective strategies to be developed for the introduction of unusual foods that are unfamiliar to a cultural group.

This thesis explores the role of culture in food acceptance by comparing two cultural contexts where insects are accepted as a regular food (i.e. northeast Thailand) and where insects are generally not eaten (i.e. the Netherlands). Based on cultural differences described by Hofstede (2001), one might expect the Dutch-being lower in avoidance of uncertainty and higher in long term orientation - to more easily accept insects as food, where its novelty and environmental benefits are likely to be attractive. Yet Dutch consumers are still largely aversive to eating insects, except for a small minority that takes interest in new meatless protein sources (House, 2016). This suggests that the cultural difference in acceptance of insects as food might be explained more by differences in the cultural setting instead of cultural values.

As Rozin (1996) argues, culture's principal effect on our food choices is by determining what foods one is exposed to in their local environment. In Thailand, a wide range of raw and cooked insects at markets and street stands are available during seasons of abundance, whereas in the Netherlands, insects have only recently been introduced for human consumption, where their availability is still confined to a few retailers that provide a limited range of species and products. Considering how consumer perceptions and rationales differ while taking into account the cultural context could provide new insights into how culture contributes to the acceptance of a food.

\subsubsection{The role of culture in food preferences}

Cultural exposure contributes to the familiarization with food items that are available for consumption and its manners of preparation and consumption (Rozin, 2003). This usually leads to certain expectations and preferences towards its use context such as how, when, where, and with whom a food is eaten (Cardello, Schutz, Snow, \& Lesher, 2000). As such, cultural preferences for certain flavours, ingredient combinations and cooking styles tend to emerge (Ahn, Ahnert, 
Bagrow, \& Barabási, 2011; Prescott, 1998). In the case of insects, preferences for certain species and preparations have been observed in places where insects are a regular food (Hanboonsong, Jamjanya, \& Durst, 2013; Luo, 1997; Obopile \& Seeletso, 2013; Yates-Doerr, 2012). Insect preparation methods in these cultures tend to adhere to cultural flavour preferences (Menzel \& d'Aluisio, 1998; Ramos-Elorduy, 1997; Yhoung-Aree, Puwastien, \& Attig, 1997). For instance, soy sauce and sugar - a typical flavour combination in Japan-are often used in the preparation of insects (Mitsuhashi, 1997).

In this respect, culture likely plays an important role in food preferences through the mechanism of exposure (Rozin, 1996). The mere-exposure effect is known to explain the liking of foods through familiarization (Zajonc, 1968), and it is through experience that children learn to eat and like certain foods that are made available to them (Birch, 1999; Nicklaus \& Issanchou, 2006). The development of specific attitudes, values and habits, can be said to be an outcome of both cultural and individual influences (Hofstede \& McCrae, 2004). Disentangling the effects of cultural exposure and individual experience might help to achieve a better understanding of culture's specific role in influencing food preferences.

\subsection{TASTE AS A DETERMINANT OF ACCEPTANCE}

Eating is a frequent activity that is very much driven by pleasure (Rappoport, Peters, Huff-Corzine, \& Downey, 1992; Westenhoefer \& Pudel, 1993). Hence, the taste of a food is considered by many to be a strong determinant of food choice decisions (Mela, 2001b; Tuorila, 2007). To date, studies investigating the consumer acceptance of novel foods tend to directly compare the liking of foods differing in familiarity and conclude that novel foods have less sensory appeal than familiar foods (Raudenbush \& Frank, 1999; Tuorila, Lahteenmaki, Pohjalainen, \& Lotti, 2001). Such comparisons neglect that perception processes differ greatly before and after experiencing a food (Grunert, Bredahl, \& Bruns $\varnothing$, 2004). Unlike familiar foods, novel foods are evaluated based on limited relevant knowledge regarding the experience qualities of a product. Moreover, Martins and Pliner (2005) show that the willingness to eat a novel food depends more on the level of interest and disgust than the expected taste of the food. Consumers' inherent tendency to show both interest and hesitation towards novel foods (Rozin \& Vollmecke, 1986) further complicates studies of novel food acceptance, where initial trial of the food might be determined more by how an individual responds to the uncertainty rather than by how much they like the taste of the food.

\subsubsection{The role of sensory-liking and food appropriateness}

Sensory-liking of a food (i.e. hedonic appraisal of its taste) is of key interest in food acceptance studies. Hence, sensory studies are normally conducted to quantify how much consumers like the taste of a food and link it to specific sensory properties to guide product development 
(Resurreccion, 2007; Sidel \& Stone, 1993). However, this is only meaningful for familiar foods where consumers have already developed their preferences through previous experiences with the food (Birch, 1999). In the absence of direct taste experience, examining sensory-liking is likely to be insufficient for understanding food acceptance and rejection. According to Rozin and Fallon (1987), foods can be rejected for reasons other than negative affect for the sensory properties of the food. Ideational notions relating to the nature and origin of the item, and the anticipated consequences of consumption relating to physical and psychological harm also motivate food rejections.

For culturally inappropriate foods, ideational notions play a particularly important role in its rejection (Harris, 1985; MacClancy, Henry, \& Macbeth, 2009; Pliner \& Pelchat, 1991; Rozin \& Fallon, 1987). In the case of insects, these creatures are more commonly regarded as pests and filth rather than as tasty food (Gorham, 1979; Kellert, 1993), and often evoke disgust at the prospect of consumption (Looy et al., 2014). The mere idea that insects are present within a food to be tasted could already result in its rejection, even if its properties are not perceptible (Rozin \& Fallon, 1987). Nevertheless, this does not signify that taste is irrelevant in the acceptance of culturally inappropriate foods like insects. In cultures where insects are eaten, taste is the most important driver for its consumption (Hanboonsong, 2010; Mitsuhashi, 1997; Ramos-Elorduy, 1997). Western consumers generally give little consideration to the taste of insects because they possess little knowledge and experience with eating insects (Deroy et al., 2015). It is thus important to take into account the ideational factors when assessing acceptance. Hence, this thesis explores food appropriateness (i.e. perceived fitness for consumption) in addition to sensory-liking as measures of acceptance. Exploring how the levels of sensory-liking and food appropriateness change with taste experience could provide insight into the process of learning to accept a culturally inappropriate food.

\subsubsection{Sensory perceptions of a novel food}

The lack of sensory appeal of culturally inappropriate foods has been attributed to its aversive sensory properties (e.g. slimy, mushy) (Martins \& Pliner, 2006; Tucker, 2014). Yet these reasons were given on the basis of having never tasted these foods. Studying sensory perceptions is critical to understanding food acceptance (Tuorila \& MacFie, 2007), but little has been done so far to examine the sensory perceptions of culturally inappropriate foods. The offensive nature of such foods might have made it particularly difficult to get people to taste and evaluate them. It is still not known whether the distaste of such foods is based on the consideration of the food's sensory properties, or a more general dislike of the idea of eating such foods.

Investigations of the sensory perceptions of novel foods often neglect that familiarity or productrelated experience plays an important role in perceptions and evaluations of a product (Banović, Fontes, Barreira, \& Grunert, 2012; Grunert et al., 2004; Peracchio \& Tybout, 1996). Consumers 
make inferences about the product based on its observable features and available cues (Kardes, Posavac, \& Cronley, 2004). However, inferences about the sensory qualities of a product tend to be less accurate in the absence of direct product experience as consumers lack the relevant knowledge to select and interpret cues appropriately (Alba \& Hutchinson, 1987; Dick, Chakravarti, \& Biehal, 1990). This implies that the distaste of novel foods can very well be based on misconceived beliefs about its taste, which could change once the properties of the food are experienced first-hand. That means that the experienced quality could play an important role in influencing subsequent consumption decisions (Grunert \& van Trijp, 2014). Looking into how sensory perceptions of novel foods differ before and after tasting could reveal how the sensory properties of a food play a role in acceptance, and provide insights for product development.

\subsection{PRODUCT AS A DETERMINANT OF ACCEPTANCE}

A food's preparation could strongly influence its desirability by changing its hedonic sensory qualities and perceived appropriateness (Cardello \& Maller, 1982; Mela, 2001a). Despite that, the role of the product remains relatively unexplored in novel food research. Many studies are based on consumer responses towards food names (Martins \& Pliner, 2006; Pliner \& Pelchat, 1991; Tuorila et al., 2001), which are likely to represent a general measure of an individual's preference towards the type of food (Cardello \& Maller, 1982), instead of an evaluation of the food experience. When a person has no prior experience with a food item, more abstract mental representations are formed regarding the stimuli (Bar-Anan, Liberman, \& Trope, 2006). As a result, the more specific contextual factors such as the product qualities and usage context are disregarded. Using visual presentations (e.g. images and actual products) could help to draw attention to specific product attributes, and provide a more informative view regarding consumer perceptions of culturally inappropriate foods where little is known about its taste and usage in the food context.

\subsubsection{Product development for novel foods}

Product development for novel foods usually revolves around the use of familiar flavours and preparations, and ensuring that the visual appearance of the product resembles foods that people are accustomed to (Stallberg-White \& Pliner, 1999; Tuorila, Andersson, Martikainen, \& Salovaara, 1998; Wansink, 2002). This has been consistently shown to have a positive influence on the willingness to eat unfamiliar and culturally inappropriate food items. For instance, using organ meats as fillers in sausages and ground meat products enabled its successful introduction into the American diet (Wansink, 2002).

Studies on the acceptance of insect-based products by Western consumers adopt a similar approach-disguising the physical appearance of the insects and incorporating them within familiar foods (Caparros Megido et al., 2016; Gmuer, Guth, Hartmann, \& Siegrist, 2016). While 
hiding the appearance of a novel food may help to reduce the initial aversive response, it does not guarantee repeat consumption. Consumers tend to compare novel products against what they are familiar with (Calantone, Chan, \& Cui, 2006), which implies that the addition of novel food ingredients to existing products may be perceived instead as a negative impact on the original product. Examining how the product preparation improves the willingness to eat a novel food therefore looks only at one side of the equation. In order to develop successful products, there is a need to consider how the novel product is perceived in relation to other familiar products on the market, and to understand how specific product attributes contribute to consumption intentions beyond the initial willingness to eat.

\subsubsection{The role of product appropriateness}

The effectiveness of using familiar flavours and preparations also depends on whether the combination of ingredients is perceived to be appropriate (Elzerman, Hoek, van Boekel, \& Luning, 2011; Stallberg-White \& Pliner, 1999). In this thesis, the perceived appropriateness of the product preparation in combination with the food is referred to as 'product appropriateness'. Unlike food appropriateness, which refers to whether a type of food is considered to be fit for consumption, product appropriateness concerns its usage context (Cardello et al., 2000; Schutz \& Martens, 2001), where product satisfaction depends on how the food matches with the usage situation. Product appropriateness could also refer to the food combination (Elzerman et al., 2011; Stallberg-White \& Pliner, 1999), where certain ingredients may be liked individually but disliked in combination (Cardello et al., 2000). In this case, flavour principles play a role in determining what is an appropriate combination of ingredients (Prescott, Young, Zhang, \& Cummings, 2004; Stallberg-White \& Pliner, 1999). For instance, beef may be appropriately prepared as a stew prepared for dinner, but inappropriate when combined with chocolate for dessert.

What is an appropriate way to prepare and use a food is very much influenced by individual experiences and cultural norms (Rozin, 2006), and satisfactory past experiences play an important role in determining what is considered to be appropriate (Giacalone \& Jaeger, 2016). Taking the case of insects, consumers from cultures where insects are eaten have distinct expectations regarding how various species are appropriately prepared for consumption (Hanboonsong et al., 2013; Obopile \& Seeletso, 2013). It is also observed that recipes usually fit the properties of the specific insects (Ramos-Elorduy, 1997; Yhoung-Aree et al., 1997), and this is only possible through experience and expertise with its use as a food ingredient. This is in contrast with the diverse recipes and products (e.g. soups, salads, chocolate, ice cream, lollipops) that have been created in Western cookbooks (e.g. van Huis, van Gurp, and Dicke (2012)) and novelty food retailers (e.g. Hotlix, Delibugs). There seems to be little consideration given to the distinctive tastes of the various insects species (Deroy et al., 2015), and many of these products do not match the primary motivations to use insects as a meat alternative (Shelomi, 2015). Under conditions of 
unfamiliarity, there is less clarity regarding how appropriate products can be made. There is thus a need to study consumer expectations regarding appropriate products when a food is culturally new, and how this plays a role in consumer acceptance.

\subsection{AIMS AND SCOPE OF THIS THESIS}

This thesis focuses on the case of insects and takes on an interdisciplinary approach to investigate the factors that contribute to the acceptance (and rejection) of culturally inappropriate foods. The main research question asked is "What determines the consumer acceptance of culturally inappropriate foods?", which is explored from three perspectives that give attention to the role of (1) culture, (2) taste, and (3) product. Figure 1.1 presents a schematic overview of the thesis and how the specific chapters relate to the 3 aspects.

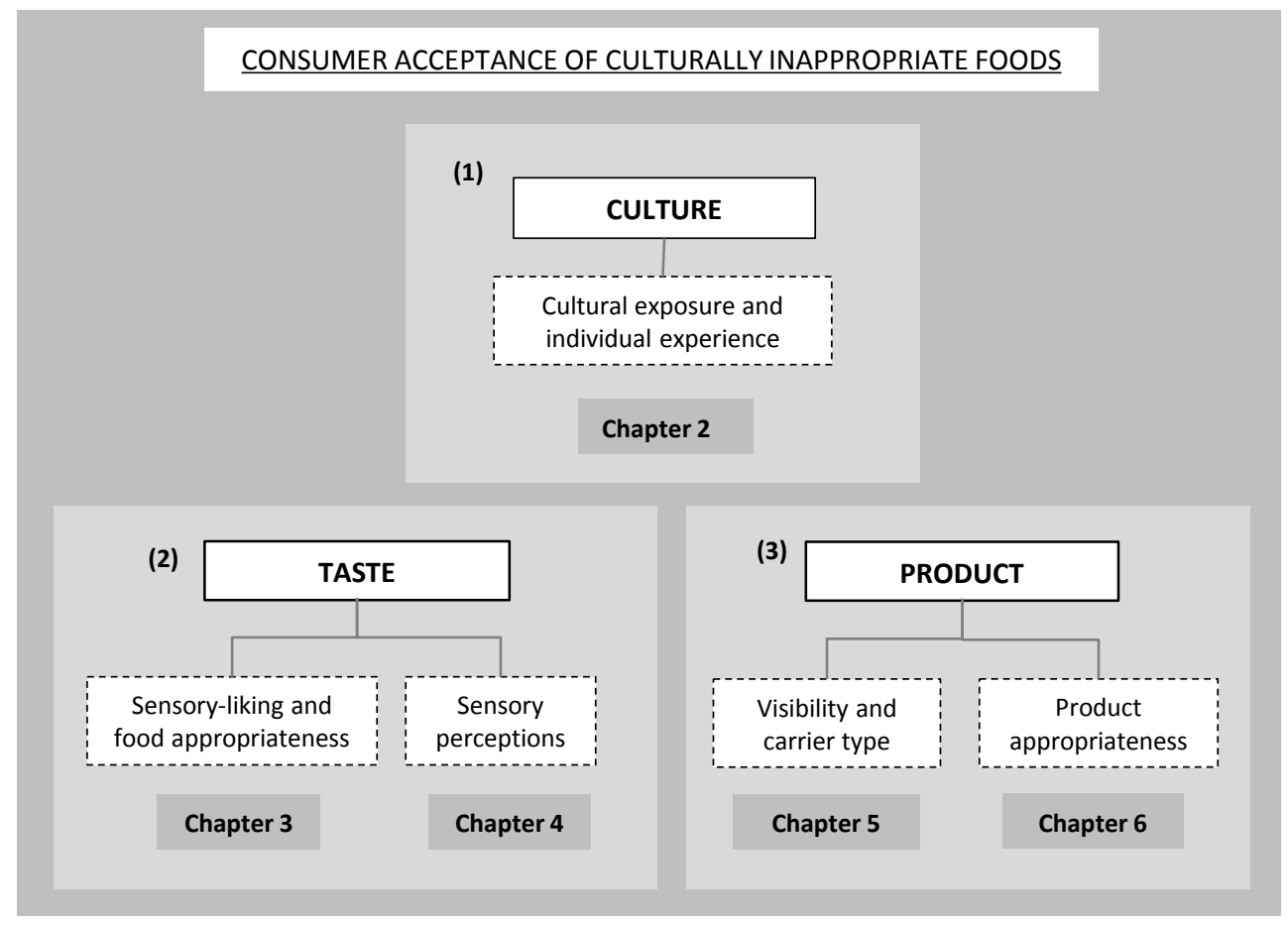

Figure 1.1. Schematic overview of this thesis

\section{(1) Culture as a determinant of acceptance}

Chapter $\mathbf{2}$ investigates how culture influences the acceptance of insects as food by comparing two cultural settings where insects are generally accepted (northeast Thailand) and rejected (the Netherlands) as food. Within each cultural setting, individuals who claim to have eaten ('eaters') 
and have not eaten ('non-eaters') insects were recruited to provide insights into how individual experiences within the cultural context play a role in acceptance. Focus group interviews were used to investigate the reasons why consumers perceive products as they do (Barrios \& Costell, 2004; Threlfall, 1999) due to its usefulness for exploring cross-cultural themes as it enables a better understanding of cultural differences (Knodel, 1995; Strickland, 1999). Semi-structured stage-wise interviews presented insects in increasing levels of contextualised detail (i.e. insects in general, species, product images, actual products for tasting) in order to explore differences in knowledge, motivations, taste-related perceptions, product preferences and behaviours between consumers who differ greatly in exposure to insects as food. This chapter identifies the factors that influence the acceptance and rejection of insects as food, and highlights the complexities and dilemmas involved when culturally inappropriate foods are considered for consumption.

\section{(2) Taste as a determinant of acceptance}

Chapter 3 and Chapter 4 study the role that taste (i.e. expected and experienced) plays in the acceptance of culturally inappropriate foods, and explores the consumer perceptions underlying the lack of sensory appeal. These chapters are based on deceptive taste experiments where Dutch consumers were led to believe that they were being served burger patties that contained unusual novel foods (i.e. mealworms, lamb brain, frog). The chapters explore how the expectations evoked by the food's identity (i.e. label) and its sensory properties (i.e. recipe) contribute towards hedonic and descriptive sensory evaluations. In chapter $\mathbf{3}$, the sensory-liking and food appropriateness were measured using Likert-type scales and their roles in determining the willingness to eat again were explored. In chapter 4 , to provide insights into the underlying sensory perceptions, consumer descriptive sensory analysis was performed using rate-all-thatapplies (RATA) method where untrained consumers select relevant sensory attributes from a checklist and rate their intensities (Ares et al., 2014). The use of consumer sensory testing in these chapters aimed at gaining insight into the extent and manner in which taste influences the acceptance of culturally inappropriate foods.

\section{(3) Product as a determinant of acceptance}

Chapter 5 and Chapter 6 investigated the impact of product characteristics on hedonic evaluations and consumption intentions. Chapter 5 is based on a survey of Dutch consumers that explores how the insect's visibility and the product type (i.e. sweet or savoury, Western or Asian) influences product acceptability through the evaluation of product images. Perceived product appropriateness, expected sensory-liking, and willingness to buy and try insect-based products were measured on Likert-type scales to gain insights into the differences between willingness to try and other measures of acceptability. Chapter 6 further investigates how product appropriateness influences the sensory-liking and willingness to buy mealworm products for trial and regular consumption with willing and unwilling tasters. Participants performed descriptive sensory 
evaluations using check-all-that-applies (CATA) (Jaeger et al., 2013) and CATA-Ideal (CATA-I) method (Bruzzone, Ares, \& Gimenez, 2012) to assess both original and mealworm meatball and dairy drink products. By systematically varying product characteristics, these chapters provide insights into how the product contributes to acceptance of a culturally inappropriate food and highlights the challenges to be overcome during product development.

Chapter 7 concludes this thesis by summarizing and integrating the key findings and discuss the implications on the consumer acceptance and product development success of culturally inappropriate foods like insects. Finally, methodological limitations and future research are discussed. 


\section{CHAPTER 2 culture's ROLE}

Insects as food: Exploring cultural exposure and individual experience as determinants of acceptance

Hui Shan Grace Tan Arnout R. H. Fischer Patcharaporn Tinchan Markus Stieger L.P.A. Steenbekkers Hans C.M. van Trijp 


\section{ABSTRACT}

Edible insects have attracted much Western interest in recent years due to their nutritional and environmental advantages. Consumers, however, remain aversive towards a class of items that is not traditionally considered to be food. While the focus is often on the Western disgust, looking at consumer perceptions in a culture that considers insects to be delicious could provide new insights into the psychological and cultural mechanisms that underpin these evaluations.

This cross-cultural qualitative study explores how cultural exposure and individual experience contribute towards the contrasting evaluations of insects as food by those who do and do not eat them. Eight focus groups were conducted across two cultures-four in Thailand where insects are part of the local food culture, and four in the Netherlands where insects are generally not recognised as food. Within these cultures, two groups consisted of individuals who have experience with eating insects, and two groups consisted of individuals with little or no experience with insects as food.

Cultural exposure created expectations of which species were more appropriate to eat and how they should be prepared, whereas individual experiences determined whether judgements were made based on memories of past eating experiences or based on the visual properties and item associations. This study provides insights into the acceptance and rejection factors of unfamiliar food items and identifies the factors to be considered when introducing novel food items that are not yet culturally acceptable as food. 


\subsection{INTRODUCTION}

Edible insects have gained much Western interest in recent years. This is due to their rich content of high quality protein and sustainability of their production in comparison to that of traditional sources of meat (Bukkens, 1997; van Huis, 2013). Yet despite much public interest in these potential benefits, most consumers in the West are still not keen on eating insects. They often react with disgust at the prospect of consuming creatures that are not familiar as food (DeFoliart, 1992; Yen, 2009), but are familiar as pests and transmitters of disease (Harris, 1985). This is in contrast with the attitudes in cultures where a variety of insect species are considered to be traditional delicacies (Hanboonsong, 2010; Ramos-Elorduy, 1997).

The importance of examining the psychological and cultural dimensions underlying attitudes towards eating insects has been emphasized before (Looy et al., 2014), but most research to date has neglected these dimensions by largely focussing on the negative Western perception and how to overcome it (Caparros Megido et al., 2014; DeFoliart, 1992; Yen, 2009). There is a lack of cross-cultural research for understanding the similarities and differences in perceptions and acceptance of insects as food. Through a qualitative research approach, the present paper aims to provide new insights on the acceptance or rejection of insects as food by exploring consumer perceptions and acceptance of edible insects across two countries where cultural exposure and individual experience with insects as food are very different.

When one looks at foods that are eaten around the world, the diversity shows that 'food' is not just for nourishment and neither is a nourishing substance necessarily 'food' (Fessler \& Navarrete, 2003; Meyer-Rochow, 2009). Research on food rejection has emphasized the physical nature of the food item and their meanings and functionalities for a particular cultural group (Fessler \& Navarrete, 2003; Martins \& Pliner, 2006; Meyer-Rochow, 2009; Rozin \& Fallon, 1980; Turmo, 2009). Surveys within a cultural group have shown strong findings on the tendency to avoid foods such as viscera and insects due to aversive textural properties and animal associations (Martins \& Pliner, 2006). Little attention, however, has been devoted to why people in other cultures do like to eat these foods in spite of their properties.

Many psychological and biological factors govern food preferences and aversions, but while there are certain predispositions, food likes and dislikes are mostly acquired through experience (Rozin \& Vollmecke, 1986). Similarly for insects, there is no evidence of an innate aversion (Bodenheimer, 1951). Individuals learn through experience which foods are appropriate to eat and how they are appropriately eaten (Cardello \& Schutz, 1996). Children are taught at a young age what not to eat (Rozin \& Vollmecke, 1986), and at the same time also learn to like specific foods through mere exposure and social learning (Nicklaus \& Issanchou, 2006). This suggests that both cultural exposure and individual experience would play an important role, where locals learn from a young age to accept foods that are available in their culture. 
Food choice usually takes place within a range of familiar foods (Prescott, 1998), where taste satisfaction is a key driver for food choice (Roininen, Lahteenmaki, \& Tuorila, 1999; Tepper \& Trail, 1998). However, prior experience is also a pre-requisite for the formation of sensory preferences (Einstein \& Hornstein, 1970). When prior experience is absent, the willingness to try is often dependent on the level of interest and disgust rather than on the expected experience of the sensory properties (Martins \& Pliner, 2005). We posit that the positive and negative evaluations of insects as food could be explained by the different levels of individual experience with eating insects, as prior experience is known to play an important role in how products are perceived, due to the moderating effect of knowledge on how stimuli are interpreted and evaluated (Banović et al., 2012; Olson, 1978; Peracchio \& Tybout, 1996; Steenkamp, 1990).

Prior to tasting, consumers make inferences about a product's properties based on their knowledge and form certain product beliefs based on the observed stimuli (Kardes et al., 2004). Consumers draw different inferences according to their familiarity with the products, where high levels of experience with eating a product result in inferences that predict the actual sensory properties more accurately, and low levels of experience result in less relevant inferences being drawn (Alba \& Hutchinson, 1987; Dick et al., 1990). Nevertheless, consumers tend to act according to their beliefs about a food item regardless of whether they reflect the actual sensory experience (Steenkamp, 1990). Food could thus be rejected on the basis of inaccurate presumptions of bad taste (Rozin \& Fallon, 1980, 1987), often resulting in the rejection of items that evoke disgust before it is even tasted. This is especially relevant in the case of insects where negative associations are prevalent.

Knowledge about taste and usage of a food item are limited when prior experience is absent. This leads to a reliance on what is known about the category in order to form a judgment (Bar-Anan et al., 2006). In the case of insects in Western culture, this alludes to the frequent associations of insects as pests and disease-transmitters. On the contrary, high levels of prior experience enable the recall of past experiences in contextualized detail even without being presented with visual cues (Bar-Anan et al., 2006). Hence, when individual experience is limited, detailed visual presentation in a food context could play an important role because it provides a concrete representation of the item (Trope, Liberman, \& Wakslak, 2007) and gives much information on what can be expected of its sensory properties (Yeomans, Chambers, Blumenthal, \& Blake, 2008). Therefore going through increasingly contextualised images and actual products containing a variety of insect species could provide several layers of insights into consumer perceptions and expectations of insects as food.

In the current study, focus group discussions were utilised to explore the different consumer perceptions and expectations regarding insects as food in two different cultural contexts one where insects are eaten (Thailand), and one where insects are generally not eaten (The Netherlands). Within each culture, groups of people that differed in levels of eating experience 
were selected. Through structured discussions about insects as food and images of various species and insect-based products, the effects of cultural exposure and individual experiences on consumer perceptions were examined. This cross-cultural study provides qualitative insights into consumer rationales behind food acceptance and rejection factors and the complex considerations involved in the acceptance of novel food items that are not yet culturally acceptable as food.

\subsection{MATERIALS AND METHODS}

\subsubsection{Methodology}

Focus group interviews were used as the method of data collection in this study. It is an explorative qualitative research tool that is widely used in consumer research to investigate reasons why consumers perceive products as they do (Barrios \& Costell, 2004; Threlfall, 1999). Focus group interviews are structured discussions usually involving several groups of 4-10 people nonrandomly selected from the target population. Group interaction allows for greater accessibility of shared ideas (Morgan \& Spanish, 1984). This methodology is particularly useful in cases where little is known about a topic (Krueger \& Casey, 2000) and is also often used to explore cross-cultural themes as it enables a better understanding of cultural differences (Knodel, 1995; Perrea, Grunert, \& Krystallis, 2015; Strickland, 1999). With respect to insects, focus groups could provide insights into the differences in consumer perceptions and expectations in two cultures that perceive insects very differently.

\subsubsection{Participants and study design}

Eight focus group interviews were conducted-four in Wageningen, a town in The Netherlands where insects are available for human consumption in specialty shops and events but are generally not recognized as food, and four in Sakon Nakhon, a province in Thailand where the consumption of insects is part of the local culture. Within each culture, two levels of individual experience were represented, each with two groups of 'eaters' who have eaten insects before and two groups of 'non-eaters' who claim not to eat insects. In total, 54 participants (19 males, 35 females) with a mean age of 38 years (20-65 years) were interviewed in eight groups of 6-8 participants. Each group consisted of participants of similar levels of insect-eating experience, but were a mix of genders, ages, education levels and occupations. There were 15 Dutch noneaters (D/NE; 7 males, 8 females), 14 Dutch eaters (D/E; 4 males, 10 females), 12 Thai non-eaters (T/NE; 5 males, 7 females) and 13 Thai eaters (T/E; 3 males, 10 females). Due to the differences in cultural availability of insects as food, equivalent experiences for eater and non-eater groups across cultures could not be achieved, and this was taken into account during the interpretation of results. 
The study was advertised via emails and social media in the Netherlands, and via door-to-door visits in Thailand. Participants were invited to participate in a group discussion on edible insects. They were screened on their prior experiences with eating insects and food allergies in order to exclude those with food allergies and to assign target participants to the relevant 'eater' or 'noneater' groups. Ethical approval was granted and all participants gave written consent.

\subsubsection{Structure of the interview}

A semi-structured focus group protocol was conducted in four stages of discussion: 1) individual experiences and knowledge, 2) reasons to eat or not eat insects, 3) evaluation of images of insect species and products, and 4) optional tasting and evaluation of insect-based products. In order to ensure commitment to individual opinions, participants first recorded their personal responses in individual booklets in stages 2 and 3, and were asked to read it out to the group before group discussion took place. The protocol and response booklet were developed in English and tested with two focus groups: 4 Dutch students and 4 Thai students (all from Wageningen University). Minor adjustments were made with regards to the labelling of pictures and the phrasing of questions in order to achieve cultural equivalence in meanings. The English protocol was then translated into Dutch and Thai, and translation quality was checked by means of back-translations. The Thai protocol was additionally tested with 4 persons in Sakon Nakhon, in order to check the understanding of questions within the local context. The interviews were conducted in the native language of the participants and were moderated by native speakers of the local language. The first author was present as an observer in all sessions in order to ensure consistency in the way the focus groups were conducted.

\subsubsection{Choice of insects in images and for tasting}

The availability of various edible insect species in Thailand and the Netherlands were determined by surveying the local distribution points in the Netherlands and in Sakon Nakhon, Thailand. Eight edible insect species were selected to include species that are both locally available and not locally available in the Netherlands and Thailand (Table 2.1). The eight selected species varied in stages of life cycle, size, colour and form to stimulate discussion about a variety of species properties. These were photographed on a serving dish without any accompanying ingredients and the images were displayed without the name of the species.

Six insect-based products were selected to include familiar and unfamiliar species incorporated in sweet and savoury products at three levels of visibility - whole and visible, whole and coated, ground and invisible (Table 2.2). These products were photographed and images were displayed to participants with a short description to inform participants about aspects of the product that might not be interpretable from the image alone. Five insect-based products, purchased from various commercial sources in Thailand and the Netherlands, were selected to include familiar 
Table 2.1. Stimulus images of familiar and unfamiliar edible insect species.

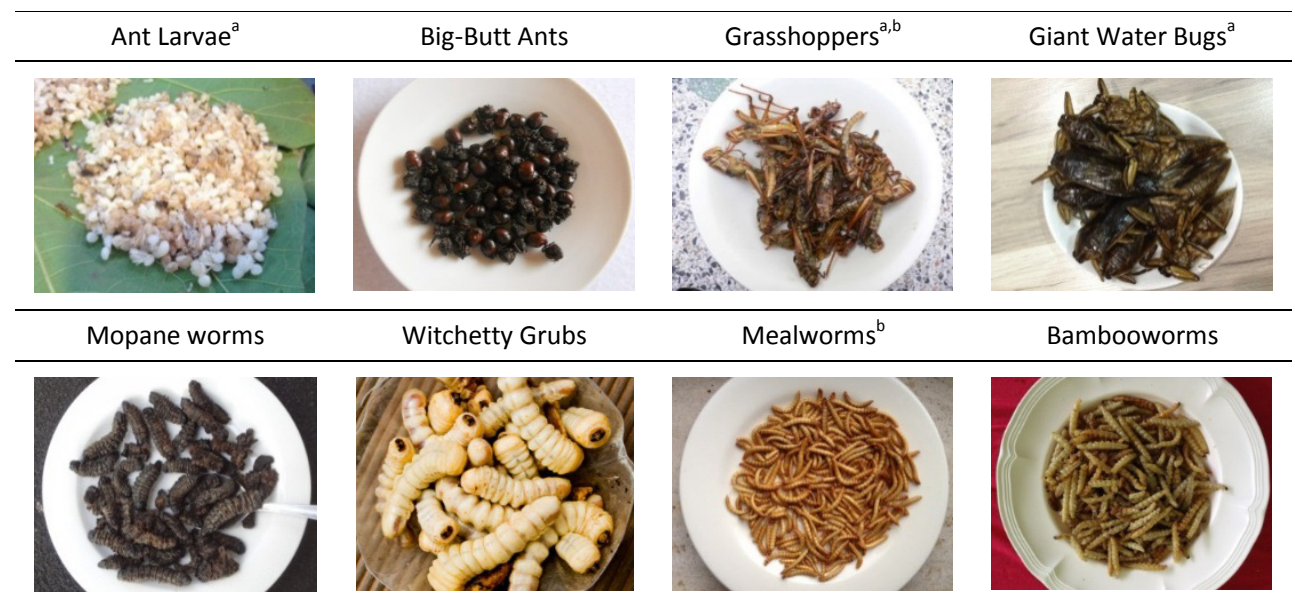

${ }^{a}$ Species available as food in Sakon Nakhon, Thailand. ${ }^{b}$ Species available as food in Wageningen, The Netherlands. Names of species were not presented with the images.

Table 2.2. Stimulus images of insect-based products with product descriptions as given in focus groups.

\begin{tabular}{|c|c|c|c|}
\hline & Visible & Covered & Invisible \\
\hline $\begin{array}{l}\text { Product } \\
\text { descriptions: }\end{array}$ & $\begin{array}{l}\text { Fried grasshoppers } \\
\text { with chilli and salt }\end{array}$ & $\begin{array}{l}\text { Cricket fritters } \\
\text { with roasted peanuts }\end{array}$ & $\begin{array}{l}\text { Giant water bug } \\
\text { chilli paste }\end{array}$ \\
\hline \multicolumn{4}{|l|}{$\begin{array}{l}\text { Savoury } \\
\text { Preparations }\end{array}$} \\
\hline $\begin{array}{l}\text { Product } \\
\text { descriptions: }\end{array}$ & $\begin{array}{c}\text { Mealworm muffins } \\
\text { with chocolate pieces }\end{array}$ & $\begin{array}{c}\text { Chocolate-coated } \\
\text { grasshoppers }\end{array}$ & $\begin{array}{c}\text { Butter cookies } \\
\text { with ground beetles }\end{array}$ \\
\hline \multicolumn{4}{|c|}{ Sweet Preparations } \\
\hline
\end{tabular}

Product descriptions were presented with the images.

and unfamiliar species with different tastes and levels of visibility. These products were presented on a plate and a short explanation about the products was given.

\subsubsection{Interview procedure}

Participants were welcomed to the focus group, and the moderator addressed the ground rules of the discussion, after which participants warmed up with a round of introduction where they were asked about their individual food preferences. In stage 1, participants were asked to speak about insect species they have eaten and know are eaten, and the way in which they are prepared and consumed. In stage 2 , participants first wrote down their reasons to eat and 
not eat insects in individual response booklets, and then mentioned them to be written on the flipchart before the group discussed the reasons and their relative importance. In stage 3, eight species images were displayed all at once on the flipchart and participants were given some time to inspect the images before filling in their individual response booklets. Participants were asked to imagine that these insects are cooked but retain an appearance as shown in the pictures. The order of presentation of images was fixed on the flipchart and response booklets and was the same for all focus groups. In their response booklets, participants gave ratings of expected liking of each species on a 5-point hedonic scale (labelled: dislike very much, dislike a little, neither like nor dislike, like a little, like very much), wrote down the reason for their rating and indicated their willingness to try (yes, no, depends). The group was then asked to vote for their two mostliked and least-liked species. Vote results were recorded on the flipchart and the reasons for liking and disliking were discussed. A 10-minute break was then taken before the next set of six product images were evaluated following the same procedure. In stage 4, five insect-based products were presented for optional tasting. They were given time to taste as they wish, after which, participants were asked to explain their decisions for tasting or not, and how the sensory experience compared against their expectations. The session was concluded with participants writing down in their response booklets their ideal recipe or the best way that they envision insects to be prepared. They were then invited to share any additional comments they have regarding the eating of insects. Each focus group lasted approximately 2 hours. At the end of the interview, participants received a monetary incentive equivalent to 2 hours of skilled work in the local context as a token of appreciation.

\subsubsection{Data analysis}

Focus group interviews were audio-recorded and transcribed verbatim by native speakers of Thai and Dutch. Note takers and observers made field notes recording non-verbal expressions and actions during the group interviews. Individual responses were recorded in response booklets, and both transcripts and individual responses were translated into English and checked by a second person for translation-accuracy.

Transcripts were content-analysed using ATLAS.Ti7.0, coded by stages of the interview and grouped by participant group types (D/NE, D/E, T/NE and T/E). Evaluations on species and insectbased products were coded according to positive or negative valence and codes were grouped according to the item being evaluated. Results are presented and discussed according to the structure of the interview, in the order of a) cultural and individual factors (introduction round, stage 1 and stage 2), b) evaluations prior to tasting (stage 3), and c) evaluations after tasting (stage 4). Due to time constraints of the focus group interviews, participants did not discuss the ideal insect-based recipes that they had written down, hence this was not analysed in the results. Response book ratings of expected liking and the willingness to try the 8 species and 6 insectbased products were averaged within participant groups. 


\subsection{RESULTS}

\subsubsection{Cultural and individual factors}

\subsubsection{Participant food preferences}

In the introduction round, participants spoke about their general dietary preferences (e.g. 'try new foods', 'prefer local foods') and dietary restrictions (e.g. 'dislike meat', 'vegetarian'). Dutch participants (D/E and D/NE) tended to be open to trying new foods and also had a keen interest in seeking more sustainable dietary alternatives to meat. Of the 29 Dutch participants, five identified themselves as vegetarians, where one was a strict vegan and the rest were open to considering insects as food. Most of the Thai participants (T/E and T/NE) expressed a preference for local foods and a dislike for unknown or 'strange' foods.

\subsubsection{Experiences and knowledge of insects as food}

Differences in cultural exposure and individual experiences with insects as food resulted in differing knowledge and experience levels across participant groups. Stage 1 focussed on two themes of discussion -a) the level of experiences and awareness of insects as food (e.g. 'eaten once', 'eat many types', 'awareness from media') and b) knowledge levels of the participants (e.g. 'preparation methods', 'sensory properties', 'lack knowledge').

\section{a) Level of experience and awareness}

Cultural exposure to insects as food in Thailand resulted in Thai participants ( $T / E$ and T/NE) having experiences or awareness of a much greater variety of insect species than the Dutch participants (D/E and D/NE). Most of the Thai experiences were gained through exposure from family and friends and from seeing insects being sold and consumed at the local markets. Thai eaters mostly grew up in Sakon Nakhon and have been consuming a variety of insects since young, whereas Thai non-eaters were either from other regions of Thailand where insects are not regularly eaten or were from Sakon Nakhon where they have tasted insects but stopped eating them due to bad taste, allergic reactions or safety concerns. It was also remarkable that some Thai non-eaters do consume certain species but do not recognize that they are insects:

"I think I have never eaten insects... but bees are considered as insect or not? The ones that come out from the beehive?... I take their honey and eat their larva."

$[\mathrm{T} / \mathrm{NE}]$

In contrast, most of the Dutch participants gained their experiences and awareness from special events, travels, and reality television programmes that had on some occasions negatively portrayed the insect-eating experience. The Dutch eaters had tasted insects once or twice during these events and travels, whereas Dutch non-eaters had never tasted insects before and gained awareness of the topic mainly through the same events and the mass media. 
Cultural exposure to insects as food also determined which species individuals considered or recognised as food (Table 2.3). Thai participants ( $T / E$ and T/NE) named a wide variety of edible species that are available within their culture, with crickets and grasshoppers being the most frequently mentioned. Due to the numerous species available, the Thai participants did not spontaneously mention some species that are commonly eaten. Local cultural practices limited the Thai experiences to several standard preparation methods such as roasting them or using them to enhance the flavour of local dishes such as chilli paste. Thai non-eaters, despite not accepting insects as food, were also aware of many of the species and preparations that Thai eaters mentioned.

Table 2.3. Frequency and types of species and preparation methods mentioned by participants.

\begin{tabular}{|c|c|c|c|c|}
\hline & $\mathrm{D} / \mathrm{NE}$ & $D / E$ & $T / N E$ & $T / E$ \\
\hline \multicolumn{5}{|l|}{ Species $^{a}$} \\
\hline Tasted (\#): & 0 & 7 & 7 & 24 \\
\hline Not tasted (\#): & 7 & 9 & 15 & 13 \\
\hline \multirow[t]{5}{*}{ Most mentions: } & Grasshoppers $(\mathrm{N}=3$ ) & Mealworms $(Y=9)$ & Crickets $(Y=4 ; N=1)$ & Crickets $(Y=9 ; N=1)$ \\
\hline & Ants $(N=3)$ & Grasshoppers $(Y=8, N=1)$ & Ant larvae $(Y=4)$ & Grasshoppers $(\mathrm{Y}=7)$ \\
\hline & Mealworms (N=2) & Crickets ( $\mathrm{N}=2$ ) & Grasshoppers $(Y=3 ; N=6)$ & Stink bugs $(Y=7)$ \\
\hline & Spiders $(\mathrm{N}=2)$ & Beetle larvae $(\mathrm{N}=2)$ & Bambooworms ( $\mathrm{N}=9$ ) & Giant water bug $(Y=6)$ \\
\hline & Crickets ( $\mathrm{N}=1$ ) & Beetles $(\mathrm{N}=2)$ & Bee larvae $(N=2)$ & Small crickets $(Y=5)$ \\
\hline \multicolumn{5}{|l|}{ Preparations $^{\text {b }}$} \\
\hline Tasted (\#): & 0 & 12 & 6 & 8 \\
\hline Not tasted (\#): & 1 & 0 & 7 & 5 \\
\hline \multirow[t]{5}{*}{ Most mentions: } & Fried $(\mathrm{N}=1)$ & Quiche $(Y=3)$ & Fried $(\mathrm{Y}=3, \mathrm{~N}=10)$ & Roasted $(Y=15, N=6)$ \\
\hline & & Pastry $(\mathrm{Y}=2)$ & Roasted $(Y=2, N=5)$ & Soup $(Y=6, N=1)$ \\
\hline & & Cookies $(Y=1)$ & Omelette $(\mathrm{Y}=2)$ & Chilli paste $(Y=5)$ \\
\hline & & Chocolate $(\mathrm{Y}=1)$ & $\operatorname{Raw}(\mathrm{Y}=1, \mathrm{~N}=3)$ & Fried $(Y=4, N=2)$ \\
\hline & & $\operatorname{Dried}(\mathrm{Y}=1)$ & Steamed $(N=3)$ & $\operatorname{Raw}(\mathrm{Y}=3)$ \\
\hline
\end{tabular}

${ }^{a}$ Species named per participant group with most frequently named examples of species that have been tasted

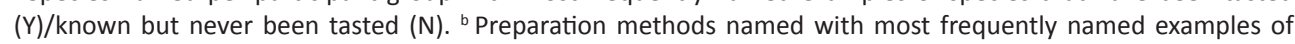
preparation methods of species that have been tasted (Y)/known but never been tasted (N). Participant group types are abbreviated as: D/NE - Dutch non-eaters, D/E - Dutch eaters, T/NE - Thai non-eaters, T/E - Thai eaters.

On the other hand, Dutch participants (D/E and D/NE) named a different set of species, where species mentions were mostly limited to mealworms and grasshoppers-the two species that were recently made available for human consumption in the Netherlands. The lack of cultural practices of eating insects led to the Dutch eaters recalling unique instances of having tasted insects, which resulted in a considerable number of preparation methods mentioned with little repetition across participants. The Dutch non-eaters were hardly aware of how insects are prepared. 


\section{b) Knowledge levels}

Cultural exposure resulted in Thai participants (T/E and T/NE) having much more knowledge about the methods of preparation than the Dutch participants (D/E and D/NE), and individual experiences resulted in eaters having more knowledge about the preparations and sensory properties than the non-eaters. Thai eaters, who were regular eaters of insects, were highly knowledgeable about the characteristics of the different species and had distinct preferences. They differentiated well between the tastes of different species:

"Each insect has a different taste, some insects have a savoury taste, some insects have a bit of meat, some insects are soft and creamy... Each insect tastes different, not like pork or chicken."

In addition, Thai eaters also possessed much knowledge of how cooking influences the sensory properties because of cumulative experiences of preparing and consuming insects with their families since young. They expressed clear expectations of how insects should be prepared in order to taste best:

"When they are fried in hot oil they will be expanded and crispy which is different from boiled or steamed insects. If you boil or steam insects, they will become watery and soft and stick in your teeth..."

Thai non-eaters were aware of common preparation methods but were not knowledgeable about the sensory properties as they lacked eating experiences. Dutch eaters, who lack cultural exposure and have limited eating experience, did not express specific preferences and struggled to recall the sensory properties:

"I didn't think it's a clear taste... It wasn't that I really liked it but it also wasn't very nasty."

$[\mathrm{D} / \mathrm{E}]$

Dutch non-eaters, having no experience with insects as food, had no knowledge about their sensory properties.

\subsubsection{Interest and concerns regarding insects as food}

In stage 2, asking about the reasons to eat or not eat insects resulted in discussions regarding their a) interest and motivations, and b) concerns and criteria when considering insects as food. The reasons to eat are summarized in Table 2.4, where experience-related codes pertained to past experiences of insects as food, situation-related codes pertained to occasions that participants could imagine eating insects, and product-related codes pertained to product attributes that would attract or deter one from considering insects as food. The reasons not to eat were not tabulated as they reflected similar attitudes but framed differently (e.g. 'if tasty' versus 'not tasty'). 


\section{a) Interest and motivations}

Cultural exposure and individual experience resulted in differences in overall interest and reasons for considering insects as food. Experience-related reasons such as cultural and family acceptance of insects as food and past positive taste experiences were often mentioned by the Thai participants ( $T / E$ and $T / N E$ ), and absent amongst the Dutch participants ( $D / E$ and $D / N E$ ). Thai non-eaters were much less interested than the Thai eaters and mentioned few reasons to eat insects, as they have earlier rejected insects as food. The tastiness of insects was a particularly important motivation amongst the Thai eaters who reflected mostly on their positive eating experiences and gave reasons that related to their tastiness and how to enjoy them:

"I eat insects because I use it as cooking ingredient for enhancing flavour. For example, bamboo soup with insects is more delicious than without insects."

Table 2.4. Reasons to eat insects.

\begin{tabular}{|c|c|c|c|c|}
\hline & \multicolumn{4}{|c|}{ Frequency of codes per group } \\
\hline & $\mathrm{D} / \mathrm{NE}$ & $D / E$ & $\mathrm{~T} / \mathrm{NE}$ & $\mathrm{T} / \mathrm{E}$ \\
\hline Experience-related codes & 0 & 0 & 7 & 29 \\
\hline Culture & 0 & 0 & 5 & 5 \\
\hline Family & 0 & 0 & 0 & 5 \\
\hline Tasty & 0 & 0 & 2 & 19 \\
\hline Situation-related codes & 11 & 8 & 9 & 0 \\
\hline Curious trying & 5 & 7 & 6 & 0 \\
\hline To survive & 4 & 1 & 1 & 0 \\
\hline Social pressure & 2 & 0 & 2 & 0 \\
\hline Product-related codes & 29 & 29 & 6 & 16 \\
\hline Sustainable & 6 & 6 & 0 & 0 \\
\hline Healthy & 5 & 5 & 2 & 3 \\
\hline Practical benefits & 0 & 0 & 2 & 11 \\
\hline New experience & 9 & 8 & 0 & 2 \\
\hline If tasty & 0 & 2 & 0 & 0 \\
\hline If practical & 5 & 7 & 0 & 0 \\
\hline If not visible & 4 & 1 & 2 & 0 \\
\hline Total number of codes & 40 & 37 & 22 & 45 \\
\hline
\end{tabular}

Participant group types are abbreviated as: D/NE - Dutch non-eaters, D/E - Dutch eaters, T/NE - Thai non-eaters, $\mathrm{T} / \mathrm{E}$ - Thai eaters.

When probed further about the benefits of insect consumption, the Thai eaters emphasized that it is about the taste pleasure rather than the extrinsic benefits:

"Do they have benefit or not? ... I eat insects because they are delicious and I enjoy eating them. Everyone knows that pleasure and satisfaction of the stomach, right? That's all. Delicious."

Dutch participants ( $D / E$ and $D / N E$ ), on the other hand, despite not having the same cultural exposure to insects as food, demonstrated an unusually high level of interest as they offered 
many reasons to eat insects. Their interest was mainly motivated by the novelty of the experience and the purported environmental and health benefits of insect consumption. Their interest related to the openness to trying new foods and interest of many Dutch participants in seeking sustainable and nutritious alternatives to meat. These sustainability reasons were unique to the Dutch participants, who frequently spoke about insects as a good source of protein that can be used as an alternative to meat:

"[The] most important reason that I would eat insects is indeed that protein. I have quit eating meat because I just find it quite taxing on the environment...that's why I would think it's a good alternative."

\section{b) Concerns and criteria}

Cultural exposure and individual experience also resulted in differences when participants discussed their reasons not to eat insects. Thai participants (T/E and T/NE) spoke about their dislike of consuming species that they do not normally eat, often mentioning the fear of negative consequences and the lack of experience, which related to the dislike of new foods by the Thai participants. In contrast, despite the high level of interest by the Dutch participants (D/E and D/ $\mathrm{NE})$, they were uneasy about the idea of eating insects and uncertain about the benefits, where they often mentioned a lack of knowledge on the production, safety, preparation and availability as reasons not to eat insects. Where individual experience with eating insects was low (T/NE and $D / E$ ) or absent ( $D / N E)$, participants spoke frequently of criteria that have to first be fulfilled in order to accept insects as food. This included product criteria (e.g. 'if unrecognisable') and situations where there is little choice but to eat insects (e.g. 'if starving'):

"I will eat insects if I didn't see their shape. And if I didn't have anything else to eat, I will eat insects even though it is the whole insect. Imagine that I get lost in the forest and I will eat insects to keep me alive."

\subsubsection{Evaluations prior to tasting}

In stage 3, discussions of the reasons for liking or disliking various species and insect-based products revealed how items were interpreted differently as a result of differences in familiarity with the items, and subsequently evaluated against different expectations that stem from cultural exposure and individual preferences.

\subsubsection{Evaluation of species}

Expected liking and willingness to try the 8 insect species in the absence of a recipe context differed across participant groups and species (Table 2.5). Discussions on their reasons for liking or disliking showed that differences in cultural exposure and individual experience influenced a) species preferences and $b$ ) the basis upon which the evaluations were made. 


\section{a) Species preferences}

Cultural exposure resulted in similar species being preferred within cultures, but different species being preferred by each cultural group. Thai participants (T/E and T/NE) were the least negative about ant larvae, grasshoppers and giant water bugs-the three species that

Table 2.5. Expected liking and willingness to try different species.

\begin{tabular}{|c|c|c|c|c|c|c|c|c|}
\hline & \multicolumn{2}{|c|}{$\mathrm{D} / \mathrm{NE}$} & \multicolumn{2}{|c|}{$\mathrm{D} / \mathrm{E}$} & \multicolumn{2}{|c|}{ T/NE } & \multicolumn{2}{|c|}{ T/E } \\
\hline & Liking & WTT & Liking & WTT & Liking & WTT & Liking & WTT \\
\hline Ant larvae & - & + & - & + & + & + & ++ & ++ \\
\hline Big-butt ants & - & -- & - & - & - & - & - & + \\
\hline Grasshoppers & - & - & - & + & - & - & ++ & ++ \\
\hline Giant water bugs & - & - & - & - & - & - & ++ & ++ \\
\hline Mopane worms & - & - & - & - & - & - & - & - \\
\hline Witchetty grubs & - & - & - & - & - & - & - & -- \\
\hline Mealworms & - & - & - & + & - & - & -- & - \\
\hline Bambooworms & - & - & - & + & - & - & - & - \\
\hline
\end{tabular}

Liking: ranged from -2 (dislike very much) to +2 (like very much). $(++)$ : mean $>1 ;(+): 0<$ mean $\leq 1 ;(-):-1<$ mean $\leq 0$; $(--)$ : mean $\leq-1$. WTT: $\%$ participants who indicated a willingness to try. $(++)$ : WTT > 75\%; $(+)$ : $50 \%<$ WTT $\leq 75 \%$; $(-)$ : $25 \%<$ WTT $\leq 50 \%$; (- -): WTT $\leq 25 \%$. Participant group types are abbreviated as: D/NE - Dutch non-eaters, D/E Dutch eaters, T/NE - Thai non-eaters, T/E - Thai eaters

were available as food in the Sakon Nakhon region. High levels of individual eating experience resulted in Thai eaters showing a strong liking of these three species that they were familiar with eating. Thai non-eaters were slightly positive only towards the ant larvae, due to several participants having tasted them before. The Thai participants generally rejected the other species that have never been eaten or seen before:

"Because I never ate it before, my parents never showed me how to." [T/E on mopane worms] They were the most negative towards the worm-like mopane worms, witchetty grubs, mealworms and bambooworms, due to their resemblance to worms that they often encounter in decaying matter:

"It looks like worms in dead animal, it is rotten... I remember that image so it is not appetizing for me."

[T/E on mealworms]

On the other hand, the Dutch participants ( $D / E$ and $D / N E$ ) were the most negative towards the larger species such as giant water bugs and witchetty grubs. They did not have the abovementioned decay associations with bambooworms and mealworms, and consider them to be more appealing than the other species:

"For me it is also that they do not resemble insects... this would not really be an insect, but a worm...I don't see any wings, I don't see any crunchy outside shells, no legs indeed."

[D/NE on mealworms] 


\section{b) Basis of evaluation}

Individual experiences influenced the knowledge upon which participants based their inferences and evaluations of the species. Where a species has been eaten before, evaluations were mostly based on the expected sensory properties and informed by past sensory experiences within a specific usage context:

"It is delicious when it is cooked with food. It has a creamy liquid inside. It is not oil but it is liquid with a bit of sour taste. If we make omelette, omelette will be more delicious."

[T/E on ant larvae]

However, this does not mean that species that have been eaten before were necessarily liked as some Thai eaters also expressed their dislike of the flavour of giant water bugs. Where a species has never been eaten before, evaluations of the expected sensory properties were made based on inferences on the visible features or associations with the species:

"I think [it] is very sweet... because ants are always eating such sweet things."

[D/E on big-butt ants]

"It looks slimy and as if you feel those legs in your cheek" [D/NE on giant water bugs] Apart from the expected sensory properties, unfamiliar species were often evaluated according to whether the species was considered to be appropriate as food:

"I thought it looks like popcorn."

[D/E on ant larvae]

"I think it is gross, I find ants just gross."

[D/E on big-butt ants]

It was observed that regardless of whether beliefs about the items reflected the actual sensory properties, it influenced participants' willingness to try, where positive associations led to higher liking and willingness to try, and negative associations had an opposite effect. Where experience with a species was absent or limited, curiosity also seemed to play an important role in the willingness to try, regardless of the positive or negative beliefs about the items:

"I have never tried it, so I would like to try it."

[T/E on bambooworms]

"It is big and scary. If it is fried crispy, I would like to try it."

[T/E on witchetty grubs]

\subsubsection{Product properties and expectations}

Expected liking and willingness to try the 6 insect-based products differed across participant groups and products (Table 2.6). Discussions of the reasons for liking or disliking revealed several product-related properties and expectations that determined the overall evaluation of a product, and revealed that the preparation method could have a strong impact on the acceptance of unfamiliar food items. These product-related factors were related to (a) the perceived appropriateness of the preparation method, (b) the visibility of the insects, and (c) the expected sensory-liking of the product. 
Table 2.6. Expected liking and willingness to try insect products.

\begin{tabular}{|c|c|c|c|c|c|c|c|c|}
\hline & \multicolumn{2}{|c|}{$\mathrm{D} / \mathrm{NE}$} & \multicolumn{2}{|c|}{$D / E$} & \multicolumn{2}{|c|}{ T/NE } & \multicolumn{2}{|c|}{$\mathbf{T} / \mathbf{E}$} \\
\hline & Liking & WTT & Liking & WTT & Liking & WTT & Liking & WTT \\
\hline \multicolumn{9}{|l|}{ Ground } \\
\hline Beetle butter cookie (sweet) & + & + & + & ++ & + & + & + & ++ \\
\hline Giant water bug chilli (savoury) & + & + & + & + & + & + & ++ & ++ \\
\hline \multicolumn{9}{|l|}{ Covered } \\
\hline Chocolate grasshoppers (sweet) & - & - & - & + & - & -- & + & ++ \\
\hline Cricket fritters (savoury) & + & + & + & + & + & + & ++ & ++ \\
\hline \multicolumn{9}{|l|}{$\underline{\text { Visible }}$} \\
\hline Mealworm muffin (sweet) & - & - & - & - & -- & -- & - & - \\
\hline Fried grasshoppers (savoury) & + & + & + & + & - & - & ++ & ++ \\
\hline
\end{tabular}

\section{(a) Appropriateness of the preparation method}

Appropriateness of the preparation method referred to whether participants perceived the combination of insect, ingredients and cooking style to be fitting with one another. Presenting insects in a recipe context generally resulted in more positive evaluations than when presenting the species alone if the selected preparation method was considered to be appropriate. Cultural exposure created product expectations amongst the Thai participants ( $T / E$ and $T / N E$ ) that insects were more appropriately prepared in a savoury rather than a sweet manner:

"Normally, people eat grasshoppers in a savoury manner. Chocolate is sweet, how could they be together?"

[T/NE on chocolate-coated grasshoppers]

Although many Dutch participants expected insects to fit better with a savoury method of preparation, this did not stem from cultural exposure, but from having been introduced to insects as a sustainable meat alternative. Due to this association with meat, the addition of insects to a sweet product was thus considered to be inappropriate:

"Why should there be insects in muffins and cookies anyway. Look if they put minced meat in there, then it would also look rather strange."

[D/NE on beetle cookies and mealworm muffins]

The appearance of an insect within a product could also enhance certain negative associations. This was the case for the mealworm muffins that evoked associations with "rotten food" (T/NE) or "an apple infected by worms" (D/E). However, unexpected product combinations sometimes aroused curiosity amongst those who are open to trying new foods:

"I think it is unusual. It is [with] chocolate, I never eat [it with] chocolate, so I want to try"

[T/E on chocolate-coated grasshoppers] 


\section{(b) Insect visibility}

Reducing the visibility of the insects did not necessarily improve the liking of a product, where fried grasshoppers were preferred over chocolate-coated grasshoppers. While some participants acknowledged that concealing the insects reduced the barrier to trying, they still considered the appropriateness of the preparation method to be important to its acceptance. However, the positive scores of the beetle cookies also suggested that grinding an insect to invisibility increases acceptance and reduces the attention towards the perceived appropriateness of the combination of ingredients. This effect of reducing the visibility of the insect seemed to apply when individual eating experience with a species was low or absent. The Dutch participants ( $D / E$ and $\mathrm{D} / \mathrm{NE}$ ), and Thai non-eaters often mentioned the invisibility of insects as a reason to like the products where insects were not seen:

"It's visually appealing, because you do not see a scary creature." [D/E on beetle cookies] For the Thai eaters, invisibility was not mentioned as a reason to like these products as they are used to eating insects in whole and recognisable forms. However, when evaluating the mealworm muffin that contained an unfamiliar species, insect visibility was raised as a reason not to eat it:

"It looks like rotten food so I think if it is transformed into crush or puree, I might change my mind."

[T/E on mealworm muffin]

\section{(c) Sensory-liking of the product}

The degree of liking of the preparation method was the most frequently mentioned across participant groups and products as a factor influencing the expected liking and willingness to try. The liking of a preparation method depended on individual food preferences. Incorporating insects into a product that is liked or disliked tended to have a corresponding effect on the willingness to try:

"It's especially tasty because of the chocolate that attracts me."

[D/NE on chocolate-coated grasshoppers]

"It looks like there's a lot of chilli in it and that seems just not so tasty."

[D/E on giant water bug chilli paste]

Nevertheless, if the combination of the sensory properties of the insect and the product is not expected to be good, that could also result in a negative evaluation of the product:

"I think [when it is] coated with chocolate it will not be crispy anymore."

[T/E on chocolate-coated grasshoppers]

Regardless of whether participants have tasted a particular species or product before, they often relied upon their past experiences with the familiar ingredients or cooking styles to infer the sensory properties of the products: 
"I decided from its colour-that looks delicious and crispy."

$[\mathrm{T} / \mathrm{NE}$ on fried grasshoppers]

"It is a logical combination of textures and flavours...yes everything fits together."

[D/E on cricket fritters]

In addition, some sensory properties were not easily inferred from the image if eating experience is absent. For instance, many Dutch participants ( $D / E$ and $D / N E)$ did not expect the giant water bug to have much taste, whereas it was known by the Thai participants (T/E and T/NE) that it has a strong odour that is used to enhance the flavour of chilli paste.

\subsubsection{Evaluations after tasting}

In stage 4 where five insect-based products were offered for optional tasting, session observations and discussions were related to the decisions to taste and the evaluations of the taste experience.

\subsubsection{Decisions to taste}

Upon being presented with the insect-based products to taste, the eventual taste decision was not solely dependent on the evaluation of the expected sensory properties or perceived appropriateness. Observations and discussions reflected (a) different levels of interest and disgust, and (b) different choices of products to taste amongst the participant groups.

\section{(a) Interest and disgust}

Cultural exposure and individual experience resulted in varying levels of interest and disgust. Observations of disgust were less frequent amongst the Thai participants ( $T / E$ and $T / N E$ ) than the Dutch participants ( $D / E$ and $D / N E)$. Thai eaters spontaneously reached forward for a product of choice but the majority of Thai non-eaters were generally uninterested in tasting (session observations). Despite the earlier negative evaluations of the unfamiliar species, some Thai eaters expressed curiosity about the taste of unfamiliar insects:

"Because it is a kind of food that I would not eat very often so I would like to know how it tastes like."

Dutch participants, saw this as a rare opportunity to taste insects, with some expressing that they were excited to be able to taste insects. However, despite the high interest levels of the Dutch participants, there were many displays of disgust and hesitation with tasting (session observations). There were no observable differences between the Dutch eaters and non-eaters. Decisions to try were a mixture of interest in trying countered by fear or disgust. When interest levels were high enough to overcome the barriers to trying, a product was tasted even when it was not believed to be good:

"I'm going to [eat it] indeed, oh nasty, I'm going to indeed, oh my god...I will do it, I will eat it." $[\mathrm{D} / \mathrm{NE}]$ 
The willingness or decision to taste thus did not signify an acceptance of the product:

"Out of curiosity you are going to taste it. That does not mean the same as I'm going to eat it."

Participants employed different ways of overcoming their fears, which included closing their eyes before putting the insect into their mouth, carefully inspecting the products or asking for information from others who had tried it first. The social context played an important role, where peers provided sensory information and assurance that it was safe to eat. This helped reluctant participants to overcome their psychological barrier, where some participants stated that they might not have tasted it if not for the presence of their peers:

"I think if you all had not tasted it, then I would not have tasted the cricket for example."

$[\mathrm{D} / \mathrm{E}]$

\section{(b) Choice of product to taste}

Cultural exposure influenced which species participants were more willing to taste. Thai participants (T/E and T/NE) were most reluctant with tasting species such as mealworms while the Dutch participants (D/E and D/NE) were most reluctant to taste the crickets:

"Those crickets I have not yet been able to overcome...Those legs, yes, I would prefer to peel them."

$[\mathrm{D} / \mathrm{NE}]$

Despite having rich experiences with eating many types of insects, the Thai eaters tended to be more selective towards which species they consider to be edible:

"I'm not afraid of worms, I could touch it but I do not eat it...I think worm is not food for human."

Individual experience seemed to influence the willingness to try the visible insects, where Thai eaters who have much eating experience with crickets tended to start with tasting the whole crickets, whereas the Dutch participants and Thai non-eaters tended to start with the 'ground cricket' brownie.

\subsubsection{Evaluations of the taste experience}

High levels of individual experiences with eating a species resulted in the comparison of the taste of the product to sensory preferences formed from past eating experiences. This was only observed with the Thai eaters who compared the taste experience of the whole crickets in the session to the roasted crickets that they are used to eating and often came to the same evaluation:

"It is delicious but I like roasted cricket much more because cricket tastes different after mixing with seasoning. I prefer natural cricket taste."

[T/E on seasoned crickets] 
For species that have never been tasted before, participants were often surprised by their taste experience, which usually differed greatly from what they had initially imagined. Participants also had difficulty interpreting the taste of unfamiliar species. They either tried to associate the taste with familiar foods, or expressed the taste in relation to other insects within the session, or compared it against how they had initially thought it would taste like. Evaluations of unfamiliar species thus differed greatly due to an absence of common expectations, where those who expected a crispy texture of mealworms evaluated the experience positively, while others who expected it to have more a texture of meat, evaluated the crispy texture of mealworms negatively. After tasting, many Dutch participants (D/E and D/NE) remarked that they were still not ready to accept insects as regular food even though the taste was quite acceptable. Even if sensory expectations were satisfied, other practical food concerns were still a barrier to acceptance:

"Anyway it should be more available, just in the supermarket and not only in expensive specialty stores, and just less expensive."

While the Dutch participants expressed their difficulties in accepting insects as food, the Thai eaters who have accepted insects as food find it preferable to be able to taste the insect in the product:

"I think if you want to sell it you should retain its character... they have their own unique taste and flavour... insect will be forgotten like insect in cookies [chocolate], it didn't have any insect flavour."

$[\mathrm{T} / \mathrm{E}]$

\subsection{DISCUSSION}

With eight focus group discussions in two countries, the influences of cultural exposure and individual experience on the differing evaluations of various species and products were explored. The stage-wise discussions revealed that the same species and insect-based products were perceived differently and then evaluated against different sets of expectations. Outcomes showed that certain accepted foods could sometimes be rejected in other cultures, especially when the properties and nature of the items trigger associations that are inappropriate in the food context. While past discussions on food rejection often focussed on the repulsive physical properties of the food items and the cultural meanings (Fessler \& Navarrete, 2003; Martins \& Pliner, 2006), here we ventured further to explore the extent to which they are learned through cultural exposure and individual experiences.

Cultural exposure exerted its influence by making available a certain range of species and preparations to individuals within the culture, hence determining which items individuals recognise as food. In the Sakon Nakhon province of Thailand, insects have long been a part of the local cuisine, unlike in the Netherlands where insects are only recently being regarded as 
a potential food source. Having grown up in a culture where there is a rich culinary tradition surrounding insects, Thai eaters demonstrated rich knowledge of how to obtain, prepare and consume insects, and also had specific expectations with regards to which species are eaten and how they should be prepared. Thai non-eaters, despite not wanting to eat insects, have also been exposed to the same environment, and gained knowledge and expectations through indirect experiences with locals or at the markets. These shared experiences within a certain cultural context created collective expectations of what is acceptable and valued (Garro, 2000; Rozin, 1996), and can be observed from how common ingredients, combinations, preparations and eating methods define regional cuisines (Ahn et al., 2011; Nam, Jo, \& Lee, 2010). Similar distinctions between species and preparation methods are evident in other cultures where insects are eaten (Luo, 1997; Mitsuhashi, 1997).

Cultural exposure also resulted in common preferences and underlying expectations within cultures that differed between cultures. Species that were available as food tended to evoke fewer negative responses, but different species were available in each culture, resulting in the Thai participants having preferences towards different species than the Dutch participants. In addition, cultural differences in food motivations were observed (Prescott et al., 2002). Many of the Dutch participants were considering insects as food due to their motivations towards sustainable food consumption, while the Thai participants considered insects more in terms of their taste and familiarity. Culture provides the context in which individuals accumulate their experiences hence common understandings do not only apply to food items (Garro, 2000; Rozin, 2003). Thai participants were strongly repulsed by mealworms, due to the association with larvae that they often see in decaying matter- an association that was absent amongst the Dutch participants who were more familiar with mealworms as food and less familiar with the observation of larvae in decaying matter than the Thai participants. This suggests that certain food items that might be rejected because of their properties (Martins \& Pliner, 2006) could be acceptable when a different set of associations has been learned.

Individual eating experience played a key role in the liking and willingness to try. Our findings were consistent with many studies that showed that familiarity with a food item is an important determinant in the willingness to try (Martins \& Pliner, 2005; Pliner, Pelchat, \& Grabski, 1993; Tuorila et al., 2001). The outcomes of the present study showed that familiar species and products were evaluated based on both positive and negative memories of past taste experiences. Having experience with eating insects did not necessarily lead to liking, as was the case for many Thai non-eaters who rejected insects based on their own earlier bad experiences. Results confirmed that prior experience of a particular species moderated the interpretation and evaluation of stimuli (Banović et al., 2012; Steenkamp, 1990), where past eating experiences resulted in evaluations that were informed by memories of its sensory properties. In contrast, items that were unfamiliar and have never been tasted before were judged based on inferences from its visual features and categorical associations, which tended to be negative in the case of insects. 
These beliefs influenced the liking and willingness to try, regardless of whether they reflected the actual sensory experience. These observations were observed across all participant groups, although what differed were the specific associations that were made, where every individual had different learned associations depending on their cultural background and individual experiences.

Our results also highlighted a motivational difference between trying and eating. This distinction seems to be especially relevant when dealing with unfamiliar foods, where people tend to show both interest and reluctance in trying new foods (Martins \& Pliner, 2005). Thai eaters were interested because of positive past experiences with eating insects, but for the other three groups (T/NE, D/NE, D/E), the lack or absence of positive past experiences meant that decisions to taste were mainly motivated by the interest or curiosity about the new experience. In the case of the Thai non-eaters who have already rejected insects as food, the cultural abundance of insects in the food context meant that insects as food is not new to them, hence interest to taste was low. When both interest and reluctance to taste were high, as in the case of the Dutch participants ( $D / E$ and $D / N E)$, peers had a strong influence as those who tasted and encouraged others to taste too. Many participants eventually tasted products out of curiosity even if they did not consider it appropriate as food or were not expecting it to taste good. Although trying did not signify liking, it is a necessary first step to learning to like a food, where increased exposure to positive experiences with an unfamiliar food could improve its acceptance (Pliner, 1982; Tuorila et al., 2001).

Getting people to taste insects that have never been tasted before is a big challenge, as the familiarity of insects in our environment tends to evoke strong negative associations (e.g. pests, disease). These associations could be sufficient to result in rejection before tasting (Rozin \& Fallon, 1987). Nevertheless, offensive food items could be accepted if prepared in an appropriate way, as was the case with the introduction of offal in the form of sausage products (Wansink, 2002). The outcomes of this study showed that the preparation method strongly influences the expected liking and willingness to try an unfamiliar species. Reducing the visibility of the insect and incorporating it into a familiar and liked product generally improved the willingness to try an unfamiliar species. This may be due to the familiarity with certain recipe components and the overall visual appeal, which may create positive sensory expectations (Yeomans et al., 2008). An opposite effect was observed if the combination yields negative visual associations, such as mealworm muffins that resemble 'rotten food', or when it did not match consumer motivations, such as the chocolate-grasshoppers that did not fulfil the goal of eating insects for sustainability reasons. Hence, apart from expected sensory-liking of the product, other extrinsic consumer motivations could influence expectations towards which types of products are suitable for the incorporation of insects. 
The next steps in product development should take into account cultural and individual expectations towards which species should be used as food and how they should be prepared. Consumer-oriented new product development emphasizes the understanding of consumer expectations as the starting point for product creation (van Trijp \& Steenkamp, 2005), where product acceptability is not only based on the product itself, but its evaluation relative to consumer expectations within the context presented (Cardello, 1995; Meiselman, 2003). The outcomes of this study have shown that the novelty and benefits of insect consumption have generated much interest in insects as food amongst the Dutch participants. However, the cognitive arguments (e.g. health and environmental benefits) were found to be insufficient to encourage consumption. Acceptance of insects as food would require the development of appropriate products that not only lower the barriers to trying, but also taste good and deliver the benefits that consumers are interested in. This study gave indications of consumer preference towards preparing locally available insects with accompanying ingredients or recipes that are individually- and culturallyaccepted, and also expected to combine well with the sensory properties of the insects. Much work remains to be done to understand which type of products would reduce rejection and improve acceptance amongst consumers who are new to insects as food.

\subsubsection{Limitations of the research}

Cross-cultural studies pose several challenges as food values and motives vary greatly across cultures (Steptoe, Pollard, \& Wardle, 1995). The qualitative nature of the study allowed for a better understanding of cultural differences through the discussions of the reasons underlying the consumer perspectives. In this study, the Thai and Dutch participants were selected as representations of insect eating and non-eating cultures to explore the effects of cultural exposure. We expect that the cultural mechanisms described here would also apply to other cultures, although attitudes and preferences towards specific items would certainly differ per culture. The limited sample size also means that it is not possible to speculate about general population attitudes from these interviews. This was especially the case for the Dutch, where willing participants were more interested and positive towards insects as food than the general Dutch population. In addition, the focus group method brought about effects of social pressure into the discussion, which gave additional insights during the tasting of insect-based products, but makes the assessment of individual preferences more challenging. Future quantitative research could be done on a larger study population, where formal taste assessments, and psychological measures of eating behavior such as the Food Neophobia Scale (Pliner \& Hobden, 1992) and the Food Choice Questionnaire (Steptoe et al., 1995) could be administered to gain a better understanding of consumer expectations towards insects as food. 


\subsection{CONCLUSIONS}

The present study provided insights into the perceptual differences and varied expectations that underlie the contrasting evaluations of edible insects in two cultural contexts. Individual experience takes place within a cultural context where the shared availability of foods and common experiences result in the familiarity and preference towards certain species and preparation methods. Individuals perceived stimuli differently according to their knowledge and made judgments according to expectations that differed according to their cultural background and individual experiences. Due to the novelty of insects as food, Dutch consumers might be willing to try out of curiosity, but if the product does not meet expectations, it is unlikely to be accepted as food. This study provides insights into the acceptance and rejection factors of unfamiliar food items and identifies the factors to be considered when introducing novel food items that are not yet accepted as food within a culture.

\section{ACKNOWLEDGMENTS}

We gratefully acknowledge Catriona Lakemond and the team of researchers on edible insects at Wageningen University for their expertise and experiences with Dutch consumers. We thank Peter Tamas for his advice on qualitative data analysis. For their warm hospitality, assistance and sharing about the local insect-eating culture, we thank Onanong Tapanapannitikul and staff and students of the Department of Food Technology and Nutrition, Kasetsart University, Chalermphrakiat Sakon Nakhon Province Campus. 


\section{CHAPTER 3 TASTE'S ROLE}

Tasty but nasty? Exploring the role of sensory-liking and food appropriateness in the willingness to eat unusual novel foods like insects

Hui Shan Grace Tan Arnout R. H. Fischer Hans C.M. van Trijp Markus Stieger 


\section{ABSTRACT}

Unusual novel foods like insects are generally not considered to be appropriate as food in cultures where they are not commonly eaten, and are often rejected for reasons other than their intrinsic sensory properties. This study explores how the levels of sensory-liking and food appropriateness contribute to the willingness to eat unusual foods.

Dutch beef consumers ( $n=103$ ) evaluated four beef burger patties, of which one was labelled to contain only beef, and the other three labelled to contain beef and unusual novel ingredients (lamb brain, frog meat, mealworms). Four different recipes were randomly presented with each of the four labels, where novel ingredients were not actually present, and instead plant-based ingredients were used to create sensory differences between formulations.

Before tasting, sensory-liking and food appropriateness of the novel burgers were lower than that of the beef burger. After tasting, the level of sensory-liking of the novel burgers increased to a similar level as that of the beef burger, whereas the level of food appropriateness of the novel burgers remained lower than that of the beef burger. Future willingness to eat remained lower than that of the beef burger. Willingness to eat again was predicted mainly by food appropriateness, and not by the experienced sensory-liking nor the individual traits (food neophobia, gender).

We conclude that positive sensory experiences play a necessary role in the process of learning to accept a food, but are inadequate when unusual and culturally inappropriate foods are involved. As curious tasting does not imply acceptance, we recommend that efforts to introduce unusual novel foods like insects not only focus on increasing trial probability, but also on understanding consumer expectations regarding their consumption. 


\subsection{INTRODUCTION}

Insects are highly valued as tasty and nutritious food in cultures where they are commonly consumed (Hanboonsong, 2010; Ramos-Elorduy et al., 1997). In contrast, most Western consumers do not share this appreciation for insects as food. While scientists have established insects to be, in principle, an excellent source of high quality protein that can be more sustainably produced than traditional livestock (DeFoliart, 1997; van Huis, 2013), most Western consumers are still not keen to adopt insects into their regular diets despite an awareness of the benefits (Chapter 2; Verbeke, 2015). Deeply entrenched attitudes, food-related concerns, and sociocultural norms still stand in the way of the acceptance of insects as food (Lensvelt \& Steenbekkers, 2014; Looy et al., 2014; Shelomi, 2015).

Changing attitudes towards unappealing foods is challenging but not impossible (Rozin \& Vollmecke, 1986). The goal of this study is to provide further insights into the process of learning to appreciate an unusual novel food when initial perceptions are strongly negative. Rational arguments have proven to be ineffective in increasing the consumption of sustainable and healthy foods if the foods are not expected to taste good (van Trijp \& Fischer, 2011; Verbeke, 2006). Sensory-driven approaches have thus been emphasized to be of key importance when attempting to change attitudes towards novel foods (Arvola, Lähteenmäki, \& Tuorila, 1999; Deroy et al., 2015; Wansink, 2002). While the role of cultural, psychological and food dimensions underlying novel food rejections have been frequently discussed (Martins \& Pliner, 2006; Rozin \& Fallon, 1980), little is known about whether negative attitudes towards the more unusual novel foods could be overcome by positive sensory exposure. This research aims to draw upon existing knowledge regarding novel food acceptance and rejection to explore the potential for unusual foods to be introduced in cultures where they are not commonly eaten.

Food acceptance and rejections are motivated by a combination of sensory-affective reasons (relating to the sensory properties), ideational notions (relating to the nature and origin) and safety concerns (relating to physical or psychological harm) (Rozin \& Fallon, 1987). The degree to which these motivations contribute to the food choice decision depend very much on the type of food and level of familiarity (Martins \& Pliner, 2005; Pliner \& Pelchat, 1991), as well as the cultural context (Chapter 2; Hartmann et al., 2015). Items that are not regarded as food in a culture are said to be 'inappropriate' and can be rejected due to the perceived associations (Rozin \& Fallon, 1987). Certain culturally-inappropriate foods like insects and other uncommonlyeaten animals (e.g. horse, dogs) and animal parts (e.g. viscera) (Harris, 1985; Hopkins, 2014) may evoke disgust at the prospect of consumption, and be perceived to be less tasty and safe to eat (Rozin \& Fallon, 1987). In this study, we explore the acceptability of unusual novel foods by measuring the changes in the level of sensory-liking (i.e. hedonic appraisal of the food's sensory properties), which relate more to sensory-affective motivations, and the changes in the level of 
food appropriateness (i.e. perceived suitability for consumption), which may relate more to the ideational and safety motivations.

Sensory-liking usually plays a major role in food choice decisions (Mela, 2001b; Tuorila, 2007), consistently ranking above other extrinsic motivations (e.g. health and environmental concern) (Steptoe et al., 1995). For that reason, sensory-liking is typically the focus of studies on food acceptance (Resurreccion, 2007; Sidel \& Stone, 1993). However, where unusual animal-based foods are concerned, examining sensory-liking is likely to be insufficient for understanding their acceptability as food, as reasons for rejection are often due to other non-sensory associations (Martins \& Pliner, 2005; Pliner \& Pelchat, 1991). In the case of insects, unfamiliar species could sometimes be judged without consideration of their intrinsic sensory properties (e.g. simply gross), although some consumers still make inferences about its sensory properties based on the nature or appearance of the species (Chapter 2). We posit that apart from sensory-liking, investigating the perceived food appropriateness could provide a better understanding of the acceptance of unusual novel foods.

Earlier studies have consistently shown that the expected sensory-liking of novel foods is often less positive than that of familiar foods (Arvola et al., 1999; Raudenbush \& Frank, 1999). This may relate to the absence of positive memories of the food or feelings of uncertainty when it has never been tasted before. It is thus more relevant to consider whether novel foods could gain acceptance after tasting. Knowledge regarding a food's sensory properties increase with taste experience, where consumers increasingly base their evaluations on the recall of past sensory experiences, rather than on the existing associations with the nature and appearance of the item (Chapter 2). Taste exposure thus often improves the sensory-liking of novel foods (Pliner, 1982), although the effects are not always effective for novel foods with a strong initial dislike (Pliner \& Pelchat, 1991; Tuorila et al., 2001). Taste exposure is nevertheless a required first step for learning the sensory properties of a food and for developing sensory preferences. Hence, examining the effects of taste exposure would provide practical insights regarding the process of how food preferences are developed.

Introducing novel foods to consumers is likely to pose several challenges, largely due to the uncertainty and less positive expectations that contribute to low consumption intentions (Arvola et al., 1999; Raudenbush \& Frank, 1999; Tuorila, Meiselman, Bell, Cardello, \& Johnson, 1994). Consumers tend to base their decisions on their pre-consumption beliefs (Steenkamp, 1990), which could result in the rejection of potentially tasty and beneficial food due to various misconceptions. Consumer's expectations are also known to influence their perceptions of the food, with most food expectation studies showing evidence that consumers may evaluate foods in the direction of their expectations (Piqueras-Fiszman \& Spence, 2015; Schifferstein, 2001). This could have negative implications on the acceptance of unusual foods that are expected to taste bad, as these expectations could influence the subsequent sensory-liking of the food when it is tasted. This raises the question of whether initially 'nasty' foods could eventually be 'tasty'. 
Consumers' inherent tendency to show interest and hesitation towards novel foods (Rozin \& Vollmecke, 1986) adds a layer of complexity to studies of novel food acceptance. In the case of insects, Dutch and Belgian consumers' willingness to taste insects due to their curiosity and interest in the environmental benefits (Chapter 2; Caparros Megido et al., 2014) may facilitate its introduction as food. However, their unwillingness to adopt insects into the existing diet despite their interest (Chapter 2; Verbeke, 2015) highlights the need to consider intentions of repeat consumption after tasting. In addition, the willingness to eat novel foods could also be dependent on individual traits such as food neophobia and gender, where more neophilic and male Belgian consumers were observed to express higher willingness to adopt insects as food (Verbeke, 2015). Yet strong rejections of culturally-inappropriate foods across entire populations suggest that these individual traits may play a relatively minor role in comparison to consumer preferences when determining behaviours towards unusual foods.

Practically-speaking, lowering the initial barriers of consumption is a critical step for the introduction of novel and unappealing foods (Wansink, 2002). Many studies have examined various techniques for improving the initial willingness to taste novel foods (Martins, Pelchat, \& Pliner, 1997; Tuorila, Andersson, et al., 1998). Familiar flavours or preparations could improve the willingness to taste (Loewen \& Pliner, 1999; Pliner \& Stallberg-White, 2000; Wansink, 2002) but may have limited effectiveness for items that evoke disgust (Martins et al., 1997; Pliner, 1982). In such cases involving disgust, the item could instead be perceived to 'contaminate' the food it is incorporated in (Rozin \& Fallon, 1987). Consumers could nevertheless become more receptive to tasting unusual and unappealing foods if they are not visually perceptible and presented in a familiar preparation (Chapter 2; Caparros Megido et al., 2014; Wansink, 2002). We apply these lessons to investigate the potential for unusual foods to gain consumer acceptance if they were to resemble a familiar and liked food.

This study investigates the influence of taste exposure on the levels of sensory-liking and food appropriateness and their subsequent influence on willingness to eat unusual novel foods. The study also explores the effects of food neophobia and gender in the evaluations. In order to extend the study of unusual foods beyond the case of insects, we selected three edible animal species (lamb brain, frog meat, mealworms) that are not often encountered as food in the Netherlands. Due to the strong aversions and safety concerns regarding these foods, we chose not to incorporate the actual ingredients in the foods to be tasted, but to lead participants to believe that the foods have been partially substituted with these unusual ingredients by labelling them accordingly. This deception improves the generalizability of the study findings by enabling the randomization of label-recipe combinations for separating the effects of recipe and label, without causing participants to unknowingly ingest ingredients that they may not want to eat. Moreover, the deception permitted the recruitment of participants without pre-informing them of the prospective consumption of unusual ingredients, which avoids a selection bias of the participants towards highly adventurous consumers. 


\subsection{MATERIALS AND METHODS}

This study is part of a larger study on consumer expectations and perceptions of unusual novel foods. In the current paper, 103 Dutch participants performed hedonic evaluations (sensoryliking) of beef-based burger patties labelled to contain beef only or a combination of beef and unusual novel ingredients. A group of another 100 Dutch participants performed a descriptive sensory evaluation of the flavour and texture attributes of the same stimuli, of which a limited number of outcomes will be briefly mentioned in this paper. For brevity, 'burger' is used in this paper to denote the meat patty of a burger, without the bun or other accompanying condiments. We also refer to the burgers labelled to contain the unusual novel ingredients to be 'novel burgers' and the ingredients themselves to be 'novel ingredients'. The burger labelled to contain beef only is referred to as 'beef burger'.

\subsubsection{Study design}

Four label descriptions $(100 \%$ beef or $75 \%$ beef and $25 \%$ lamb brain, $25 \%$ frog meat or $25 \%$ mealworms) were presented in combination with four beef-based burgers. The three novel ingredients were not actually incorporated into the samples. Instead, plant-based ingredients (breadcrumbs, tofu, hazelnut) were added in sufficient amounts to create perceivable differences in taste and texture, in order to improve the credibility of the label information, and to explore the effects of the experienced sensory differences on the sensory-liking and food appropriateness of the burgers. Participants were randomly assigned to one of four groups (Table 3.1) that received a set of four label-recipe combinations consisting of the four labels matched with a different recipe. Between the four groups of participants, all 16 combinations ( 4 labels $\times 4$ recipes) were evaluated an equal number of times. This allowed the examination of main effects and interactions of the label and recipe on the burger evaluations.

Table 3.1. Recipe-label combinations assigned to the four participant groups.

\begin{tabular}{lcccc}
\hline \multirow{2}{*}{ Participant } & \multicolumn{4}{c}{ Label } \\
\cline { 2 - 5 } & 'Beef burger' & 'Lamb brain burger' & 'Frog meat burger' & 'Mealworm burger' \\
\hline Group 1 & Recipe A & Recipe B & Recipe C & Recipe D \\
Group 2 & Recipe B & Recipe C & Recipe D & Recipe A \\
Group 3 & Recipe C & Recipe D & Recipe A & Recipe B \\
Group 4 & Recipe D & Recipe A & Recipe B & Recipe C \\
\hline
\end{tabular}

Each group consisted of 25 or 26 participants. Beef burger was described to contain $100 \%$ beef as the meat component, and novel burgers were described to consist of $75 \%$ ground beef and $25 \%$ ground novel ingredient.

\subsubsection{Participants}

A total of 103 participants (62 males, 41 females; $22.9 \pm 5.5$ years) participated in the study. Recruitment was done in the vicinity of Utrecht University (The Netherlands) and the study 
was advertised for participants of Dutch nationality who have neither food allergies nor food restrictions and who like to eat hamburgers. Participants were recruited without pre-informing them of the prospective consumption of unusual foods, so as to avoid selection bias towards highly adventurous participants. Participants were informed during the session that tasting was optional whenever the novel burger labels were presented. Ethical approval was granted and all participants gave written consent.

\subsubsection{Label selection}

Selection of food labels was based on two preliminary surveys conducted with 19 and 12 Dutch university students who reported their familiarity, perceived food appropriateness, and expected sensory properties of 12 novel ingredients that are available in the Netherlands but not commonly eaten. They also asked the same questions regarding burgers that have been incorporated with these ingredients. Lamb brain, frog and mealworms were selected for this study, as participants perceived them at varying levels of food appropriateness, and expected them to have a sensory impact when incorporated into beef burgers. Results from the descriptive sensory evaluation showed that sensory expectations of each burger and ingredient differed significantly from the others on multiple flavour and texture attributes, confirming that the labels evoked unique expectations for the stimuli (data not shown).

\subsubsection{Recipe formulation}

A basic burger recipe consisting of $0.5 \%$ salt and $0.4 \%$ ground black pepper added to ground beef (purchased at a local supermarket) was modified to create four burger recipes that were visually similar but perceptibly different in flavour and texture. The four recipe formulations are shown in Table 3.2. Small amounts of each ingredient were added to all the burgers to minimize visual differences between the burgers. Results from the descriptive sensory evaluation showed that sensory perceptions of each burger recipes upon tasting differed significantly from the others on multiple flavour and texture attributes, confirming that the burger recipes were perceived to be different by the participants of this study (data not shown).

Table 3.2. Recipe formulations of the four beef-based burgers.

\begin{tabular}{lcccc}
\hline \multirow{2}{*}{ Recipe } & \multicolumn{4}{c}{ Ingredient Formulation } \\
\cline { 2 - 5 } & Minced beef (\%) & Breadcrumbs (\%) & Tofu (\%) & Hazelnuts (\%) \\
\hline Recipe A & 94 & 2 & 2 & 2 \\
Recipe B & 86 & 10 & 2 & 2 \\
Recipe C & 80 & 2 & 16 & 2 \\
Recipe D & 88 & 2 & 2 & 8 \\
\hline
\end{tabular}

All burgers were seasoned with $0.5 \%$ salt and $0.4 \%$ ground black pepper. 


\subsubsection{Sample preparation}

Fifty gram burgers were cooked on medium heat in a frying pan for about $15 \mathrm{~min}$, cooled and packed into aluminium boxes and stored at $-18^{\circ} \mathrm{C}$ in the week prior to the study. Fifteen minutes before each session, frozen burgers were defrosted in the microwave for 30 s and heated in an oven at $200^{\circ} \mathrm{C}$ for $5 \mathrm{~min}$. They were kept warm in heated porcelain dishes wrapped in aluminium foil. Samples were served to participants within $20 \mathrm{~min}$ of reheating. Sample trays were labelled with random 3-digit codes that were matched according to the codes and labels within the questionnaires.

\subsubsection{Test procedures}

\subsubsection{Session procedures}

Participants attended a single tasting session, conducted between $10.30-20.00 \mathrm{~h}$ over two weeks in a room in the psychology department of Utrecht University. The room for observational studies was furnished like a dining room, and partitions were placed on the dining table to create sensory booths. Participants filled in consent forms prior to the session and were asked to clean their palate with crackers and water before and between tasting each sample. Each session lasted 30-45 minutes. Participants received 7 euros as a reward upon completing the questionnaire. All participants were debriefed at the end of each session, during which they were informed that the novel ingredients were not actually present and participants were asked to refrain from telling about the study to others until the end of the test period.

\subsubsection{Acceptability measures}

Sensory-liking before and after tasting were assessed with the questions "How tasty do you think this burger would be?" (before tasting), and "How tasty did you find this burger?" (after tasting) and rated on a 9-point Likert scale, verbally anchored from 'extremely not tasty' to 'extremely tasty'. Food appropriateness was assessed by asking "How appropriate do you think this burger is for consumption?", and rated on a 9-point Likert scale verbally anchored from 'extremely inappropriate' to 'extremely appropriate'. Willingness to eat was assessed by asking "How willing are you to eat these burgers in the following situations?" and rated on a 5-point Likert scale verbally anchored at the ends with 'not willing at all' and 'extremely willing'.

\subsubsection{Questionnaire design}

The four-part questionnaire was designed in English and translated into Dutch by a native speaker. Part 1 involved beef burger evaluations. Prior to tasting, an image of a grilled beef burger patty was presented with the instruction to imagine tasting such a ground beef burger. Participants first evaluated their levels of expected sensory-liking, and then they were served one of the 
burger recipes according to their assigned set of label-recipe combinations (according to Table 1) and evaluated their perceived sensory-liking of the burger tasted.

At the start of Part 2, participants were informed that they will be asked to imagine the taste of some burgers that may seem somewhat strange but are potential innovations for the future. They were presented with the same image of a grilled beef burger patty as in Part 1 and asked to imagine how three burgers with meat contents consisting of $75 \%$ ground beef and $25 \%$ ground lamb brain, frog meat, or mealworms would taste, and evaluate their expected sensory-liking. The presentation order of the novel burger labels was randomized to control for order effects. Food appropriateness of beef and the three novel burgers were then evaluated.

Part 3 involved an optional tasting of the novel burgers and began with the instruction that if participants prefer not to taste a certain burger, that they could check the box indicating that 'I have not tasted it' and proceed with the questionnaire. Participants were served their assigned burger recipes for each novel burger label in the questionnaire (according to Table 1) and evaluated the perceived sensory-liking of the burgers. The presentation order of the 3 novel burgers was the same as in part 3. Food appropriateness of beef and the three novel burgers was evaluated again.

In part 4, participants were asked to imagine that they have the possibility to eat the burgers in three situations (meal, special occasion, snack), and to evaluate their willingness to eat the burgers, as well as the four ingredients themselves (beef, lamb brain, frog meat, mealworms) when not ground and incorporated into a burger. The four ingredients were then evaluated on expected sensory-liking and food appropriateness. Participants then reported their familiarity with the four ingredients as food on a 5-point category scale (1-not known as food, 2- known but never tasted, 3-tasted before, 4-eat occasionally, 5-eat regularly), and described their experiences with the novel ingredients if they had tasted them before. Lastly, they filled in the Food Neophobia Scale (FNS) (Pliner \& Hobden, 1992) and explained their reasons if they had rejected tasting a particular novel burger. Age and gender were also reported.

\subsubsection{Data analysis}

Responses on sensory-liking, food appropriateness and future willingness to eat were transformed into numbers: 1-9 for sensory-liking and food appropriateness, and 1-5 for future willingness to eat. Ratings of FNS were reversed on items 1, 4, 6, 9 and 10, and the sum of scores are reported according to Pliner and Hobden (1992). Ratings of sensory-liking and food appropriateness of ingredients were analysed between familiarity levels (ingredients grouped) and within familiarity levels (across ingredients) using one-way analysis of variance (ANOVA). Effects of label and recipe on the evaluations of sensory-liking and food appropriateness before and after tasting burgers were analysed using two-way ANOVA (4 labels $x 4$ recipes), with gender and FNS as covariates. Changes in levels of sensory-liking and food appropriateness after tasting were analysed using 
paired samples t-tests. Missing values due to the rejection of burgers were excluded on a case analysis basis. Future willingness to eat was analysed by repeated measures three-way ANOVA (4 labels $\times 3$ situations $\times 2$ forms of preparation), with gender and FNS as covariates. Bonferroni corrections were applied for all post-hoc tests. The relations between sensory-liking, food appropriateness, future willingness to eat and FNS were analysed using Pearson's productmoment correlations. Hierarchical linear regressions were performed to assess the predictive ability of gender, FNS and perceived sensory-liking and food appropriateness after tasting on the willingness to eat the burgers again in future in a regular meal situation.

\subsection{RESULTS}

\subsubsection{Role of ingredient familiarity}

\subsubsection{Ingredient familiarity}

Participants were all regular or occasional consumers of beef, but had no or limited (one to a few tastings) experiences with the novel ingredients as food (Figure 3.1a). According to the claims of the 103 participants, $7.8 \%(n=8)$ have tasted lamb brain during their travels, 36.9\% $(n=38)$ have tasted frog meat mostly in Chinese restaurants in the Netherlands, and $22.3 \%(n=23)$ have tasted mealworms at promotional events in the Netherlands. These experiences with novel ingredients were in a variety of preparations but not in the form of burgers. In addition, lamb brain ( $n=41)$ and mealworms $(n=41)$ were less known as food than frog meat $(n=14)$.

\subsubsection{Sensory-liking and food appropriateness across familiarity levels}

Ingredient familiarity had a significant effect on the levels of expected sensory-liking $[F(4,407)=142.07, p<0.001]$ and food appropriateness $[F(4,407)=83.43, p<0.001]$ of the ingredients, where each step higher in familiarity resulted in more positive evaluations for both measures (Figure 3.1b). This meant that consumption frequency, degree of consumption experience, having a taste experience, and being aware of a food's edibility all contributed to the degree of the ingredient's acceptability. Correlations between the expected sensory-liking and food appropriateness were highly positive and significant $(p<0.001)$ for all ingredient labels [Pearson's $r=+0.50$ (beef), +0.53 (lamb brain), +0.70 (frog), and +0.63 (mealworm)], indicating a strong relationship between these two measures when a preparation context was not specified. 


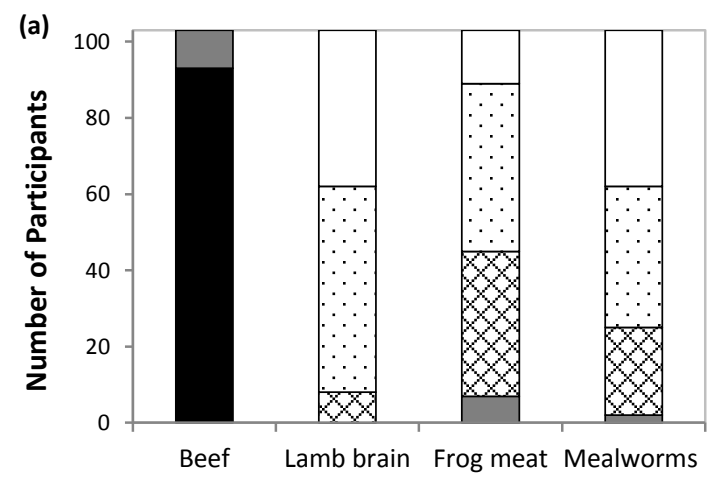

\author{
$\square$ Eat regularly \\ $\square$ Eat occasionally \\ $\square$ Tasted before \\ $\square$ Known as food, never tasted \\ $\square$ Not known as food
}

$\square$ Eat regularly

$\square$ Eat occasionally

$\square$ Tasted before

$\square$ Known as food, never tasted

$\square$ Not known as food

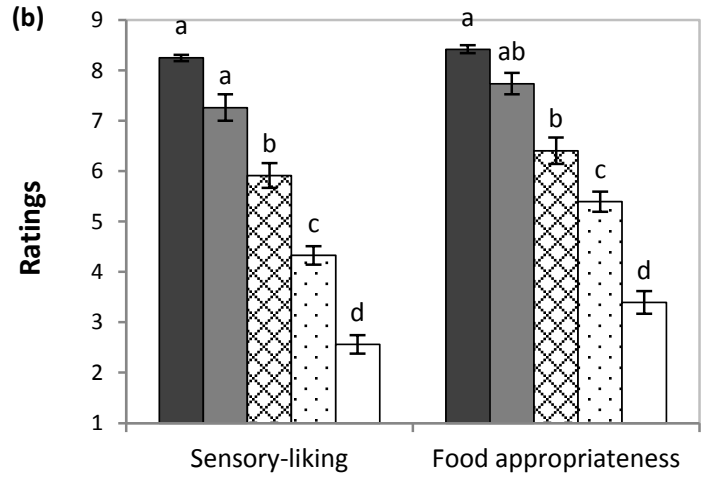

Figure 3.1. Ingredient familiarity and the effects on ingredient evaluations. (a) Number of participants per familiarity level for each ingredient. (b) Mean ratings of sensory-liking and food appropriateness grouped across ingredients and split according to familiarity levels. Error bars represent standard errors and different letters represent significant differences between means ( $p<0.05$, Bonferroni).

\subsubsection{Sensory-liking and food appropriateness within a familiarity level}

The levels of expected sensory-liking and food appropriateness differed between novel ingredients when they were compared at the same level of familiarity (Figure 3.2). There were no significant differences in the expected sensory-liking $[F(2,93)=2.32, p=0.10]$ and food appropriateness $[F(2,93)=2.71, p=0.72]$ when the ingredients were not known as food. When edibility is recognized, significant differences between the novel ingredients were found for food appropriateness $[F(2,132)=4.78, p=0.01]$, where lamb brain was considered to be less appropriate for consumption than the other two ingredients, but there were no significant differences in the expected sensory-liking of the novel ingredients at this level of familiarity $[F(2,132)=0.43, p=0.65]$. When there has been taste exposure, the level of expected sensoryliking differed significantly between novel ingredients $[F(2,66)=10.68, p<0.001]$ but not for food appropriateness $[F(2,66)=2.33, p=0.11]$, which suggests that limited taste exposure may have had stronger effects on sensory-liking than food appropriateness. 

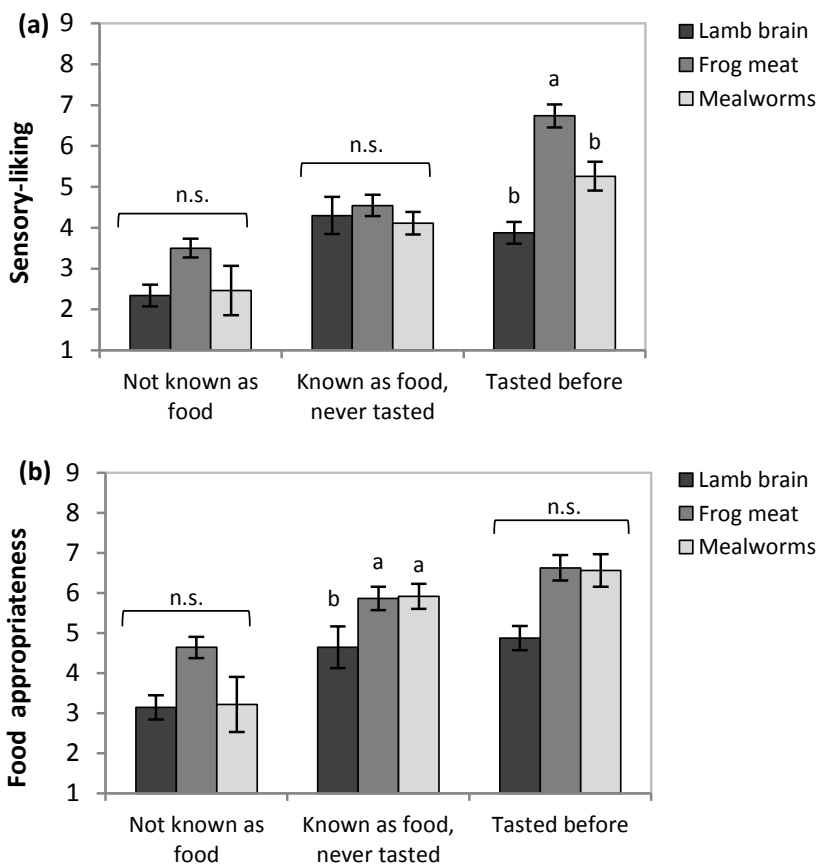

Figure 3.2. Evaluations of novel ingredients within familiarity levels. Mean ratings of (a) sensory-liking and (b) food appropriateness per familiarity level are shown, error bars represent standard errors, n.s. indicates no significant differences between novel ingredients, and different letters indicate significant differences between novel ingredients within the familiarity level ( $p<0.05$, Bonferroni).

\subsubsection{Sensory-liking and food appropriateness before and after tasting}

\subsubsection{Novel burger rejections}

Almost all participants $(94 \%, n=97)$ tasted all three novel burgers, with only 4 rejections of burgers labelled to contain lamb brain, 1 rejection of a burger labelled to contain frog meat and 1 rejection of a burger labelled to contain mealworms. Two participants mentioned safety concerns regarding the consumption of lamb brain, whereas the other rejection reasons pertained to feeling uneasy with the idea of consuming these novel ingredients. None mentioned negative expectations of sensory-liking as the reason. It was also observed during the session that a number of participants were hesitant during tasting and expressed these feelings during the post-study debriefing, indicating that some participants were willing to set aside their negativity to taste the novel burgers.

\subsubsection{Sensory-liking and food appropriateness before and after tasting}

The main effects and interactions of label and recipe on the levels of sensory-liking and food appropriateness before and after tasting are reported in Table 3.3. Prior to tasting, label 
significantly influenced both expected sensory-liking and food appropriateness, where novel burgers were rated significantly less positively than the beef burger (Figure 3.3). Sensory-liking and food appropriateness of the novel burgers were strongly correlated before tasting [Pearson's $r=+0.54$ (lamb brain), +0.71 (frog meat), +0.71 (mealworm), all $p<0.001$ ]. Food appropriateness of beef burger was not measured prior to tasting. No significant recipe main effect nor label-recipe interaction effect before tasting confirms that expectations did not differ between participant groups.

Table 3.3. Effects of label and recipe on sensory-liking and food appropriateness before and after tasting.

\begin{tabular}{|c|c|c|c|c|c|c|c|c|}
\hline & \multicolumn{4}{|c|}{ Before Tasting } & \multicolumn{4}{|c|}{ After Tasting } \\
\hline & $\mathrm{df}$ & $\mathrm{F}$ & $\mathrm{p}$ & $n_{p}^{2}$ & $\mathrm{df}$ & $\mathrm{F}$ & $\mathrm{p}$ & $\eta_{p}^{2}$ \\
\hline \multicolumn{9}{|l|}{ Sensory-Liking } \\
\hline Label & 3 & 46.25 & $* * *$ & 0.26 & 3 & 1.12 & n.s. & 0.01 \\
\hline Recipe & 3 & 0.48 & n.s. & 0.00 & 3 & 8.11 & $* * *$ & 0.06 \\
\hline Label*Recipe & 9 & 0.73 & n.s. & 0.02 & 9 & 2.93 & $* *$ & 0.06 \\
\hline Gender & 1 & 29.14 & $* * *$ & 0.07 & 1 & 8.01 & $* *$ & 0.02 \\
\hline FNS & 1 & 11.79 & $* *$ & 0.03 & 1 & 9.41 & $* *$ & 0.02 \\
\hline Residuals & 394 & & & & 388 & & & \\
\hline \multicolumn{9}{|c|}{ Food Appropriateness } \\
\hline Label & 3 & 61.68 & $* * *$ & 0.32 & 3 & 34.74 & $* * *$ & 0.21 \\
\hline Recipe & 3 & 0.36 & n.s. & 0.00 & 3 & 4.18 & $* *$ & 0.03 \\
\hline Label*Recipe & 9 & 0.77 & n.s. & 0.02 & 9 & 1.32 & n.s. & 0.03 \\
\hline Gender & 1 & 28.13 & $* * *$ & 0.07 & 1 & 7.96 & $* *$ & 0.02 \\
\hline FNS & 1 & 11.10 & $* *$ & 0.03 & 1 & 11.84 & $* *$ & 0.03 \\
\hline Residuals & 394 & & & & 394 & & & \\
\hline
\end{tabular}

Two-way ANOVA's performed on the main and interaction effects of label and recipe, with gender and FNS as covariates. Significance indicated at $* * p<0.01, * * * p<0.001$, and n.s. indicates not significant $(p>0.05)$.

After tasting, label did not have a significant main effect on the level of sensory-liking, where novel burgers were evaluated to be similar in sensory-liking as the beef burger (Figure 3.3a). Instead, the perceived sensory-liking depended more on the recipe tasted. The significant labelrecipe interaction suggests that certain label-recipe combinations were perceived to be more tasty than others. Recipe effects were also significant for food appropriateness after tasting, but label effects were still dominant [partial $\eta^{2}=0.21$ ] in comparison to the recipe effect size [partial $\eta^{2}=0.03$ ], where food appropriateness of the novel burgers remained significantly less positive than the beef burger after tasting (Figure 3.3b). Sensory-liking and food appropriateness of the burgers labelled to contain novel ingredients remained strongly correlated after tasting [Pearson's $r=+0.65$ (lamb brain), +0.68 (frog meat), +0.77 (mealworm), all $p<0.001$ ] 


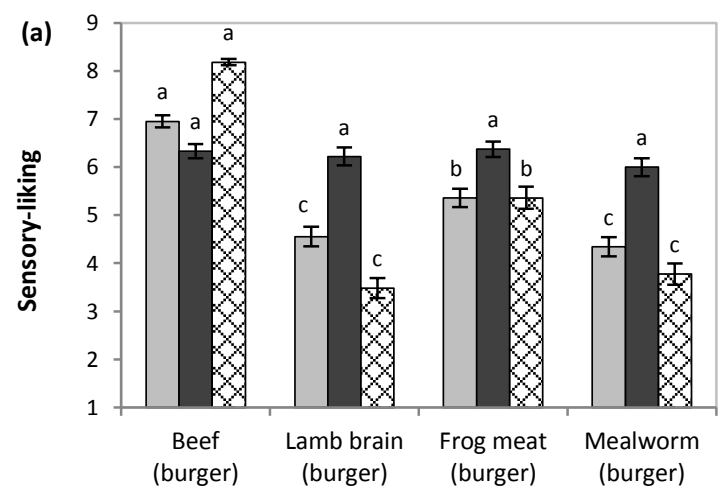

$\square$ Before (burger)
$\square$ After (burger)
$\square$ Ingredient

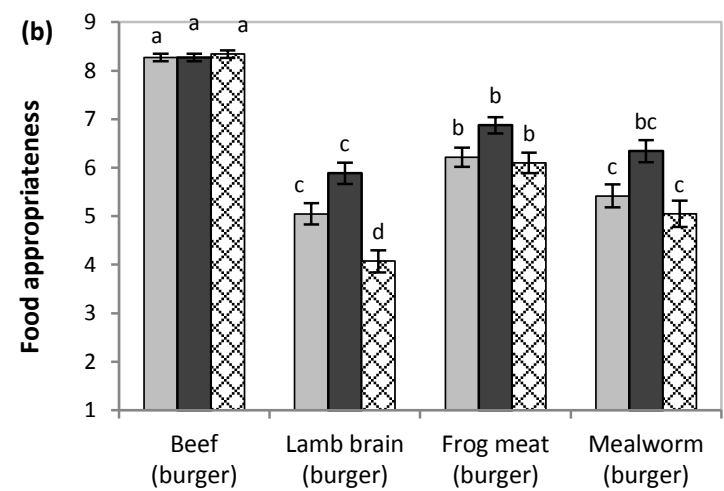

$\square$ Before (burger)

-After (burger)

$\square$ Ingredient

Figure 3.3. Ingredient evaluations and burger evaluations before and after tasting. Mean ratings of (a) sensoryliking and (b) food appropriateness are shown and error bars indicate standard errors. Two-way ANOVA was performed with gender and FNS as covariates and different letters indicate significant differences between labels per series ( $p<0.05$, Bonferroni).

\subsubsection{Change in levels of sensory-liking and food appropriateness after tasting}

After taste exposure, the level of sensory-liking dropped significantly for the beef burger $[t(102)=-3.83, p<0.001]$, but increased significantly for the burgers labelled to contain lamb brain $[t(98)=6.93, p<0.001]$, frog meat $[t(101)=4.42, p<0.001]$ and mealworms $[t(101)=8.02, p<0.001]$ (Figure 3.3a). Food appropriateness of the novel burgers also increased significantly after taste exposure for the burgers labelled to contain lamb brain [ $t(102)=4.31, p<0.001]$, frog meat $[t(102)=3.79, p<0.001]$ and mealworms $[t(102)=4.06, p<0.001]$ (Figure 3.3b). The changes in the levels of sensory-liking of the novel burgers were strongly correlated with the changes in levels of food appropriateness upon taste exposure [Pearson's $r=+0.47$ (lamb brain), +0.63 (frog meat), +0.66 (mealworm), all $p<0.001$ ]. Evaluations of the novel ingredients after tasting the burgers, however, remained equally low or significantly lower than that of the burgers before and after tasting. 


\subsubsection{Effects of individual traits on sensory-liking and food appropriateness}

Food neophobia scores (FNS) ranged from 10-42 out of the possible range of 10-70 (mean=24.4, $S D=7.2$, Cronbach's $\alpha=0.76$ ), and males and females did not significantly differ in FNS $[t(101)=-0.77, p=0.44]$. In general, more neophilic and male participants evaluated the burgers more positively on sensory-liking and food appropriateness than more neophobic and female participants. Although the food neophobia and gender effects were significant, their effect sizes were small in comparison to the label effects (Table 3.3), suggesting that individual traits played a relatively minor role in the evaluations. Effects of gender were slightly stronger before than after tasting, where the taste experience reduced the differences in evaluations between male and female participants.

\subsubsection{Willingness to eat in future situations}

\subsubsection{Effects of product context and individual traits on future willingness to eat}

The willingness to eat each ingredient, in two forms of preparation (burger vs ingredient), and in three eating situations (meal, special occasion, snack) were investigated and the main effects and interactions are reported in Table 3.4 .

Table 3.4. Effects of label, form and situation on willingness to eat.

\begin{tabular}{lccccc}
\hline & $\mathrm{df}_{\text {model }}$ & $\mathrm{df}_{\text {residuals }}$ & $\mathrm{F}$ & $\mathrm{p}$ & $\mathrm{\eta}_{\mathrm{p}}{ }^{2}$ \\
\hline Label & 3 & 300 & 7.35 & $* * *$ & $\mathbf{0 . 0 7}$ \\
Form (burger vs ingredient) & 1 & 100 & 3.22 & $\mathrm{n} . \mathrm{s}$. & 0.03 \\
Situation (meal, special occasion, snack) & 2 & 200 & 8.51 & $* * *$ & $\mathbf{0 . 0 8}$ \\
& & & & \\
Label*Form & 3 & 300 & 4.60 & $* *$ & $\mathbf{0 . 0 4}$ \\
Label*Situation & 6 & 600 & 4.34 & $* * *$ & $\mathbf{0 . 0 4}$ \\
Situation*Form & 2 & 200 & 2.24 & $\mathrm{n} . \mathrm{s}$. & 0.02 \\
Label*Form*Situation & 6 & 600 & 2.55 & $*$ & $\mathbf{0 . 0 3}$ \\
Gender & & & & & \\
FNS & 1 & 100 & 15.78 & $* * *$ & $\mathbf{0 . 1 4}$ \\
\hline
\end{tabular}

Two-way ANOVA's performed on the main and interaction effects of label, form and situation, with gender and FNS as covariates. Significance indicated at $* p<0.05, * * p<0.01, * * * p<0.001$, and n.s. indicates not significant ( $p>0.05)$.

The willingness to eat novel burgers and ingredients remained significantly lower than beef burgers and beef in all three situations [label: $p<0.001$ ] (Figure 3.4). The effects of form of preparation depended on the type of burger [label*form: $p<0.01]$, where preparing the novel ingredients in a burger where it is not visually perceptible increased the willingness to eat, but had no effect on beef that is already often consumed without blending it into other foods. Similarly, the effects of situation differed between beef and novel burgers [label*situation: $p<0.001$, where the willingness to eat novel burgers and ingredients was 
generally higher in special occasions than in a regular meal or snack, but the willingness to eat beef and beef burger in a regular meal and special occasion were equally high. Willingness to eat as a snack was lower across all foods. Males and more neophilic participants were generally more positive in their evaluations of willingness to eat, where gender and food neophobia had larger or at least similar effect sizes as that of the label.

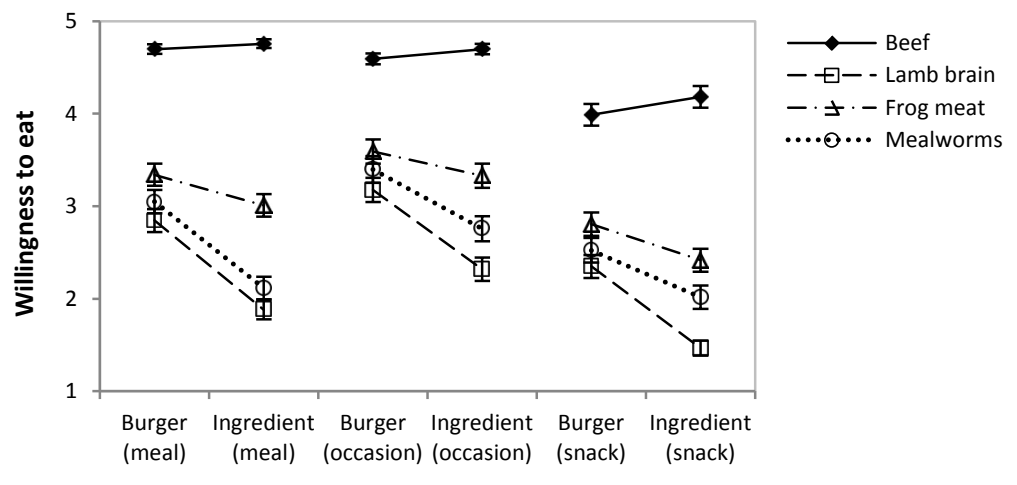

Form of preparation (Situation)

Figure 3.4. Future willingness to eat burgers and ingredients in different situations and forms of preparation. Error bars indicate standard errors.

\subsubsection{Predicting future willingness to eat in meals after the taste experience}

The role of individual traits (gender, FNS) and post-tasting evaluations of sensory-liking and food appropriateness in predicting the willingness to eat the burgers in a regular meal were examined with hierarchical regression analysis (Table 3.5).

Table 3.5. Hierarchical regression analysis of individual traits and post-tasting evaluations on willingness to eat the burgers (meal).

\begin{tabular}{|c|c|c|c|c|c|c|}
\hline \multirow[b]{2}{*}{ Model } & \multicolumn{4}{|c|}{ Standardized beta } & \multicolumn{2}{|c|}{ Model Parameters } \\
\hline & LIKE $^{a}$ & $\mathrm{APP}^{\mathrm{b}}$ & Gender & FNS & $\mathrm{R}^{2}$ & $\mathrm{R}^{2}$ change \\
\hline \multicolumn{7}{|l|}{ Beef burger } \\
\hline LIKE + APP & +0.05 & $+0.32 * *$ & & & $0.103 * *$ & \\
\hline + Gender + FNS & +0.04 & $+0.33^{* *}$ & -0.07 & +0.03 & $0.108^{*}$ & 0.005 \\
\hline \multicolumn{7}{|c|}{ Lamb brain burger } \\
\hline LIKE + APP & +0.04 & $+0.70 * * *$ & & & $0.522 * * *$ & \\
\hline + Gender + FNS & +0.03 & $+0.70 * * *$ & +0.02 & -0.02 & $0.523 * * *$ & 0.001 \\
\hline \multicolumn{7}{|l|}{ Frog meat burger } \\
\hline LIKE + APP & +0.17 & $+0.57 * * *$ & & & $0.488 * * *$ & \\
\hline + Gender + FNS & +0.16 & $+0.56 * * *$ & -0.02 & -0.04 & $0.490 * * *$ & 0.002 \\
\hline \multicolumn{7}{|c|}{ Mealworm burger } \\
\hline LIKE + APP & -0.07 & $+0.77^{* * *}$ & & & $0.517^{* * *}$ & \\
\hline + Gender + FNS & -0.08 & $+0.78 * * *$ & +0.03 & -0.04 & $0.519 * * *$ & 0.002 \\
\hline
\end{tabular}

LIKE and APP were entered in the first block, followed by gender and FNS in the second block. Significance indicated at $* p<0.05, * * p<0.01$, and $* * * p<0.001$. Bold text indicate significant values. ${ }^{a}$ LIKE denotes perceived sensoryliking. ${ }^{b}$ APP denotes food appropriateness (after tasting). 
In this study, food appropriateness was the only significant predictor of the willingness to eat beef and novel burgers as part of a regular meal. Predictions for beef burger were much weaker than for novel burgers. Adding the individual traits (gender, FNS) did not significantly improve the models for all four burgers and were individually not predictive of the willingness to eat the burgers in a meal. Where the levels of food appropriateness were the lowest llamb brain and mealworm burgers), the predictive ability of food appropriateness was the strongest. For frog meat burger that was higher in food appropriateness than the other novel burgers, the perceived sensory-liking appears to have a relatively stronger predictive ability [ $\beta=+0.16$ (frog)] in comparison to the other novel burgers [ $\beta=+0.03$ (lamb brain), -0.07 (mealworm)].

\subsection{DISCUSSION}

The role of sensory-liking and food appropriateness in the willingness to eat unusual foods was explored in this study. The study examined how food-related ideas (influenced by labels) and experienced sensory properties (influenced by recipes) influence the levels of sensory-liking and food appropriateness upon taste exposure, and investigated the subsequent impact on the future willingness to eat. While most previous research on novel food acceptance have focussed on the factors contributing to the initial willingness to try (Martins \& Pliner, 2005; Raudenbush \& Frank, 1999; Tuorila et al., 2001), here we were particularly interested in whether culturallyinappropriate foods could be accepted if sensory-liking is achieved upon tasting and what the findings mean for the introduction of unusual novel foods like insects.

Sensory-liking and food appropriateness were highly correlated before and after tasting, where the expected and experienced tastiness of the burgers are likely to have influenced its perceived appropriateness for consumption, or vice versa. This indicates that common hedonic dimensions are likely to underlie the evaluations of the foods (Cardello \& Maller, 1982). However, the levels of sensory-liking and food appropriateness were differentially influenced by the taste experience, implying that these measures did differ too in their underlying motivations. After tasting, there were no significant differences in the evaluations of sensory-liking for the burgers regardless of labels, indicating that the negative expectations of sensory-liking for the burgers labelled to contain unusual ingredients did not result in a lower sensory-liking upon tasting. This is contrary to the findings of most food expectation studies where consumers tend to evaluate foods in the same direction as their expectations (Piqueras-Fiszman \& Spence, 2015; Schifferstein, 2001). This may be due to the lack of experience with these novel foods, as consumers hold on to their expectations with less confidence when product knowledge is low (Spreng \& Page, 2001). However, food appropriateness of the novel burgers showed limited improvement after tasting and remained much lower than the beef burger. This finding provides a plausible explanation for why strongly disliked foods are less influenced by taste exposure (Pliner \& Pelchat, 1991; 
Tuorila et al., 2001), where the low perceived food appropriateness may suppress the overall liking of the food even if the taste is appreciated. Evidently, one may easily learn to like the sensory properties of a food (Birch \& Marlin, 1982; Pliner, 1982), but perceptions of a food's appropriateness for consumption seem to be more resistant to change.

After tasting, the degree of sensory-liking depended mainly on the recipe tasted rather than the label that was presented. This confirms earlier findings that the experienced sensory properties are usually the basis for evaluating sensory-liking during consumption (Cardello \& Maller, 1982; Raudenbush \& Frank, 1999). However, the preferences for certain label-recipe combinations imply that apart from the food's properties, consumer expectations also play a role when evaluating the quality of a food (Cardello \& Maller, 1982). On the other hand, food appropriateness was less affected by the taste experience, and was mainly determined by the label, with small recipe effects. This indicates that a single taste experience was insufficient to overturn the negativity towards the perceived appropriateness of these foods for consumption. Yet strong positive correlations between the change in levels of sensory-liking and of food appropriateness indicate that the quality of the taste experience is likely to play an important role in the process of learning of a food's appropriateness for consumption. This would mean that if the sensory differences between the burger recipes had resulted in greater variations in the levels of sensory-liking (i.e. from extremely good to extremely bad), then greater changes in food appropriateness of the novel burgers may have resulted from the taste experience.

Willingness to eat the burgers again in a regular meal was predicted only by food appropriateness and not the perceived sensory-liking after tasting. This is in contrast with the findings of Arvola et al. (1999) where sensory-liking of an unfamiliar cheese was a strong predictor of future intentions to purchase it. We reason that cheese being a more familiar and appropriate food, may have been evaluated more on the basis of sensory-liking (Martins \& Pliner, 2005), whereas in our study, the low perceived food appropriateness of the unusual foods may have been the stronger psychological barrier. Nevertheless, sensory-liking still plays an important role as an extremely delicious experience may influence the openness to consume the foods again, whereas a disappointing experience may reinforce the initial negativity and result in permanent rejection of the food. Considering the broader context brings to attention that the low willingness to eat the novel burgers again could be partly due to the abundance of other sources of more familiar and enjoyable foods, where participants may not perceive the necessity to consume such foods in current times, apart from satisfying their curiosity (Looy \& Wood, 2006). Considerations such as the lack of knowledge regarding its usage (e.g. preparation, safety) and other practical concerns (e.g. price, availability) may also influence the willingness to regularly consume a food that is unfamiliar in the culture (Chapter 2; Looy \& Wood, 2006). In all, our findings emphasize that mere sensory-liking of a food is important but insufficient to guarantee that unusual foods will be accepted into the existing diet, where its appropriateness as food and other contextual considerations are likely to play an important role in its adoption as food. 
Individual traits of food neophobia and gender influenced evaluations but played a relatively minor role in this study. Males and more neophilic participants generally responded more positively towards the novel burger labels, which was consistent with the findings of Verbeke (2015) who studied Belgian consumers' willingness to adopt insects as food. However, our study showed that although these individual traits significantly influenced the evaluations of sensoryliking, food appropriateness and willingness to eat, their effects were small. These individual traits did not predict the willingness to eat the novel foods in a regular meal. This result was similar to the findings of Arvola et al. (1999) who demonstrated that food neophobia was a poor predictor of purchase intentions of unfamiliar cheese despite its significant effect on evaluations. A person who is more open to trying novel foods may be more likely to taste it and learn of its properties (Tuorila et al., 2001), but ultimately sensory and ideational factors would still play the major role in the acceptance of a food. We thus recommend that greater emphasis should be placed on studying consumer preferences and expectations.

The level of expected sensory-liking and food appropriateness of the novel ingredients were dependent on the degree of familiarity and the food-specific ideas. Comparing across familiarity levels, we found that evaluations were significantly lower for participants who have merely tasted the food in comparison to occasional or regular consumers, which indicates that a certain level of taste experience is required for acceptance. The importance of distinguishing between different types of familiarity has been highlighted by Aqueveque (2015), where consumers are less willing to eat novel foods that comprise of unknown or unusual ingredients (e.g. snail soup) than novel foods that are simply new combinations of known ingredients (e.g. pineapple pizza). This study showed that in the absence of taste experience, recognition of a food's edibility resulted in more positive evaluations. In addition, where familiarity levels were low, differences in novel ingredient evaluations within the same familiarity level suggest that food-specific ideas are at play. A qualitative study has shown that the more negative the evoked associations for an unfamiliar insect species, the lower the expected sensory-liking and willingness to try (Chapter 2). These findings may explain why novel foods such as insects and organ meat that often bear strongly negative associations tend to experience stronger rejections than novel foods of plant origin that have less negative associations (Pliner \& Pelchat, 1991).

Our results highlight that consumers may be more willing to eat novel foods under certain conditions (Caparros Megido et al., 2014; Looy \& Wood, 2006). Participants expressed a higher willingness to eat the novel burgers and ingredients during more curious situations (e.g. special occasions), and expressed a lower willingness to eat in a regular meal, or as snacks, which may have been an inappropriate consumption situation for burgers and animal-based foods. The willingness to eat is also higher if novel ingredients are prepared in a burger rather than on its own. Indeed familiar preparations and reduced ingredient visibility have been repeatedly shown to have a strong positive effect on encouraging trial of insects and organ meat (Chapter 2; 
Caparros Megido et al., 2014; Wansink, 2002). However, the lower willingness to eat the burgers when labelled to contain unusual ingredients raises the question of whether consumers would actually be willing to adopt these unusual foods into their existing diets, as consumers tend to compare new products in relation to their current habits (Calantone et al., 2006).

\subsubsection{Implications of the research}

This study has shown that there is more to acceptance than the mere willingness to taste and the liking of a food's sensory properties. If consumers are to consider the regular consumption of an unusual food, not only should they be able to appreciate its taste, they should also regard it to be appropriate for consumption, otherwise interest is likely to be lost after the initial novelty has worn off. Wansink (2002) has emphasized that for the effective introduction of unappealing foods, it is important to lower the initial barriers of consumption, to change existing negative perceptions regarding the taste experience, and to also create incentives to motivate its consumption. Even though communicating consumption benefits may generally improve the initial willingness to taste, it does not change the hedonic evaluation of the food (Sabbe, Verbeke, Deliza, Matta, \& Van Damme, 2009; Stein, Nagai, Nakagawa, \& Beauchamp, 2003). The next steps in introducing novel foods should thus aim to better understand the underlying consumer expectations with regards to specific foods as opposed to general attitudes, and to direct efforts towards developing products that not only increase trial probability, but that also perform well in relation to comparable products in the existing diet.

Where unusual novel foods are involved, the strong cultural negativity and unfamiliarity with their consumption poses many additional barriers to acceptance, where the existing cultural norms and deep-seated attitudes are difficult to change with the mere awareness of its benefits or a single positive taste experience (Chapter 2; Looy \& Wood, 2006). In the case of insects, frequent promotional efforts have increased the willingness to consider eating insects in recent years (Caparros Megido et al., 2014; Verbeke, 2015), but many hurdles still remain between curiosity and actual acceptance of this unusual class of foods. Even in cultures that embrace insects as food, species that are not commonly consumed or are not perceived to be tasty do not gain popularity amongst locals (Chapter 2; Mitsuhashi, 1997). This study has provided an exploratory view of the sensory and ideational motivations underlying the negative responses towards unusual novel foods and how it changes with taste exposure. However, much work remains to be done to investigate the true potential for their acceptance as food in cultures where they are not commonly encountered as food. 


\subsubsection{Limitations and future research}

A limitation of the study is that the burgers were only labelled to contain the novel ingredients and did not actually contain them. Consequently, the quantified levels of sensory-liking do not provide a true indication of the sensory-liking of these specific burgers. In order to study the actual potential of these unusual foods to be accepted in cultures where they are not normally consumed, further studies are required to investigate the unique sensory properties of various species and preparations, and to evaluate how the sensory properties match up to consumer expectations in various consumption contexts. This would provide a more representative view of how consumers might respond to the actual consumption experience, and provide more actionable insights for the development of specific products.

Another limitation is that even though the burgers were perceptually different, larger sensory differences between the recipes could have led to a larger range of differences in perceived sensory-liking, and subsequently more drastic changes in food appropriateness and future willingness to eat as a result of the taste experience. Future studies using a range of extremely delicious to bad-tasting products would help to better the understanding of the role of sensoryliking in determining consumption intentions. Moreover, as this study was limited to three unusual animal-based foods, future research could be done with novel foods of different food categories that vary in their degree of familiarity (e.g. unknown food or unfamiliar preparation) to improve the generalizability of the findings to other novel foods.

Even though potential participants were not informed that unusual foods were involved in this study, highly neophobic participants did not take part. Mean food neophobia scores of participants ranged from 10 to 42 (mean=24.4, SD=7.2), whereas Pliner and Hobden (1992) reported a range of 10 to 68 (mean=34.5, SD=11.9) and a study on a larger Dutch population reported a range of 11 to 63 (mean=30.1, SD=9.5) (Schickenberg, Van Assema, Brug, \& De Vries, 2008). This selection bias may be due to highly neophobic people tending to avoid sensory studies (Tuorila et al., 1994) where they are likely to have to taste foods that they are not familiar with. Another possible reason could be related to the majority of participants being university students, who may be more open towards novel foods than the general Dutch population.

Conversations with participants at the end of the session also revealed that a handful of them were slightly doubtful that the burgers contained the labelled novel ingredients. However, they expressed that they were still affected by the idea, as it was still conceivable that these ingredients could be present. While most participants tasted the novel burgers, many consumed only a small quantity and were cautious during tasting. Hence, a measurement of the actual amount consumed might have been a better representation of the willingness to taste the novel burgers than the decision to taste per se. This stresses the importance for future research on novel food acceptance to incorporate measures that are more reflective of consumer attitudes towards the food, such as the measurement of actual consumption behaviour. 


\subsection{CONCLUSIONS}

The present study provided new insights into the role of sensory-liking and food appropriateness in the willingness to eat unusual novel foods, and discussed the implications on their introduction to cultures where they are not commonly consumed. Sensory-liking of a food upon tasting improves the perceived appropriateness of a food for consumption, but is insufficient for food acceptance when culturally-inappropriate foods are involved. Individual traits of food neophobia and gender played a relatively minor role in the evaluations, emphasizing the importance of studying consumer preferences in addition to individual traits. Consumers may be willing to taste unusual foods out of curiosity, but may not be willing to eat it again if the food is not regarded to be tasty nor appropriate for consumption. Efforts to introduce unusual novel foods like insects should focus on developing products that not only encourage trial but also perform well in relation to foods that are currently consumed. Furthermore, we recommend that future research on novel food acceptance includes food appropriateness as a measure of acceptability, as ideational factors and other concerns with its suitability for consumption could strongly influence its acceptance as food.

\section{ACKNOWLEDGMENTS}

We would like to express our gratitude to Floor Kroese and Zeena Harakeh of Utrecht University for providing the use of the living room laboratory that was specially designed for experimental and observational research. We also thank Claudia Tibboel for her assistance in the data collection, the students of Wageningen University for helping with the cooking, and the reviewers for helping us to improve the manuscript. 


\section{CHAPTER 4 taste's ROLE}

Why do unusual novel foods like insects lack sensory appeal?

Investigating the underlying sensory perceptions

Hui Shan Grace Tan

Claudia J. Tibboel Markus Stieger

Published in Food Quality and Preference (2017): 60, 48-58. 


\section{ABSTRACT}

Unusual novel foods like insects generally hold little sensory appeal for consumers, but little is known about the underlying sensory perceptions and how the properties of the food contribute to acceptance. As a follow up to the previous work on sensory-liking and food appropriateness, this study examined the sensory perceptions of 3 unusual novel foods (lamb brain, frog meat, mealworms) that were claimed to be present in beef burger patties.

Dutch consumers $(n=100)$ performed descriptive sensory evaluations and assessed food appropriateness of four different burger patties before and after tasting, and reported their willingness to eat again. The 4 samples were presented in different combinations with 4 labels claiming either $100 \%$ beef or $75 \%$ beef and $25 \%$ novel ingredient, to explore how the food's identity and properties contribute to sensory perceptions.

Beliefs about the food's taste were informed by species-related associations, and tended to be more negative when the food had never been tasted before. While these pre-consumption beliefs had some influence on sensory perceptions after tasting, the food's properties mainly determined the consumption experience. The low willingness to eat had more to do with food appropriateness than the actual taste of the food. The food's taste nevertheless exerts an influence on the perceived food appropriateness and should not be neglected. While incorporating unusual novel foods within a familiar product could help to create more positive expectations, it is still less appealing than the original products that consumers are familiar with. We recommend that future research on novel food acceptance should take into account that perceptions differ between novel and familiar foods, and that product development for culturally inappropriate foods requires selecting suitable product types and adjustment of its properties to match both consumer motivations and taste expectations. 


\subsection{INTRODUCTION}

Unusual novel foods like insects generally hold little sensory appeal (Chapter 2; Tucker, 2014), where the prospect of consumption often evoke feelings of disgust and distaste amongst those who are not accustomed to them as food (Martins \& Pliner, 2006; Pliner \& Pelchat, 1991; Rozin \& Fallon, 1987). In the Western context, examples of such foods include uncommon food items such as insects, frogs and snails, and animal parts such as brains, intestines and tongues (Harris, 1985; Hopkins, 2014; Rozin \& Fallon, 1980). Reasons for rejections have so far been attributed to aversive textures and their animal nature (Martins \& Pliner, 2006), whilst neglecting that these same foods are highly valued for their taste by those who eat them. This raises questions regarding the role of the food's taste in determining the willingness to eat such foods.

This paper follows up on previous work (Chapter 3) that investigated the role of sensory-liking (i.e. hedonic appraisal of the sensory properties) and food appropriateness (i.e. perceived fitness for consumption) in influencing the willingness to eat unusual novel foods. Previous findings revealed that in spite of a good taste experience, willingness to eat the novel foods again remained low due to low food appropriateness. However, strong positive correlations between sensory-liking and food appropriateness suggested that the taste of a food may still play a role in acceptance by influencing the perceived food appropriateness.

Relating consumer perceptions to food properties is critical to understanding food acceptance (Steenkamp \& van Trijp, 1996; Tuorila \& MacFie, 2007), but the sensory perceptions of novel foods are seldom investigated. Studies generally examine consumer's responses to food names and descriptions (Pliner \& Pelchat, 1991; Tuorila et al., 2001), and investigate the psychological predictors (e.g. food neophobia, risk perceptions) of willingness to eat (Baker et al., 2016; Ruby et al., 2015). While the negative bias towards novel foods is well known (Raudenbush \& Frank, 1999), little is known about the consumer perceptions that underlie this negativity and how the food's properties may influence future consumption. Following a similar experimental design of the previous work (Chapter 3), this study investigates sensory perceptions of unusual novel foods before and after tasting to provide consumer insights that can be used for the product development of culturally unusual foods like insects.

The present study draws upon elements of the Total Food Quality Model (TFQM) (Grunert et al., 2004), which integrates various theories of information-processing and decision-making to explain how quality perception processes differ before and after purchasing and trying a product. The TFQM highlights that prior to using a product, quality expectations are formed under conditions of uncertainty. This means that inferences about products are made based on incomplete information, where observable cues such as its features, description and positioning are used by consumers to extract information regarding product attributes and potential benefits (Grunert \& van Trijp, 2014; Kardes et al., 2004). Experience with the food's intrinsic qualities (e.g. taste, convenience) is only gained upon trying the product, after which, the experienced quality 
becomes important in informing future consumption decisions (Grunert \& van Trijp, 2014). Investigating how perceptions change upon tasting a food is therefore important to understanding acceptance. In addition, the experienced quality depends not only on its sensory properties, but is also influenced by prior experiences and contextual effects (Grunert et al., 2004). The lack of experience with novel foods thus adds more uncertainty to how it will be perceived upon tasting. Research on novel food acceptance usually only considers hedonic differences between novel and familiar foods (Raudenbush \& Frank, 1999; Tuorila et al., 2001). We posit that considering the underlying sensory perceptions could provide a better understanding of the negative bias towards novel foods.

Research on quality perception has mostly been done with respect to meat quality perception (e.g. beef, pork) amongst European consumers to provide insights for consumer-oriented product development in the meat sector (Bredahl, 2004; Grunert et al., 2004). While most European consumers are familiar with eating meat, they possess little knowledge and experience regarding the taste of unusual novel foods like insects. The present study explores what sensory expectations consumers have towards unusual novel foods, especially since few have ever tasted products that contain such ingredients. Consumer research have shown that product-related experience or familiarity strongly influences the way product cues are interpreted and evaluated (Banović et al., 2012; Peracchio \& Tybout, 1996). When consumers lack direct experience with a product, less relevant inferences tend to be drawn regarding the product experience (Alba \& Hutchinson, 1987; Dick et al., 1990). Even for beef, low familiarity consumers were found to be less able than high familiarity consumers to predict the experienced qualities of beef products (Grunert et al., 2004). Based on these observations, we hypothesize that sensory expectations of unusual foods differ greatly from the actual taste of the food, and differ between people who have and have not tasted them before.

During consumption of a food, the experienced sensory properties are usually the basis for evaluating sensory-liking (Cardello \& Maller, 1982; Raudenbush \& Frank, 1999). In a study of burger patties, providing information about its composition (e.g. meat- vs insect-based burger) had little effect on the sensory profiling of products, although it did affect overall liking (Schouteten et al., 2016). Outcomes from the previous study (Chapter 3) that randomised the presentation of labels and product formulations suggested that consumer expectations evoked by the food's identity may still influence the way the experienced taste is interpreted and evaluated, as certain combinations of label and product tasted were found to be preferred more than others. Understanding the sensory perceptions that underlie the hedonic evaluations and relating them to hedonic measures could provide insights regarding what consumers perceive to be tasty for products containing novel foods. This would provide indications for the product development of unusual novel foods. In the current study, we investigate the effects of the label and recipe (i.e. product formulation tasted) to explore how sensory perceptions are influenced by the food's identity and its sensory properties. 
While it is has been suggested that the acceptance of novel foods can be achieved by incorporating them within familiar products such as burger patties and sausages (Caparros Megido et al., 2016; Wansink, 2002), outcomes from our earlier studies suggest otherwise. Food appropriateness (Chapter 3) and product appropriateness (Chapter 5 \& Chapter 6) have been shown to strongly influence the willingness to eat a novel food. The taste experience could also play an important role in determining repeat consumption, where a disappointing experience usually decreases future intentions (Grunert \& van Trijp, 2014). In the previous study (Chapter 3), food appropriateness of the novel burgers increased after the taste experience, even though scores remained relatively low. The high positive correlations between sensory-liking and food appropriateness suggested that the sensory properties of the food may still play a role in the willingness to eat unusual novel foods through its influence on the perceived appropriateness of the food. The present paper thus explores the relations between sensory perceptions, food appropriateness and willingness to eat, in order to gain insights into how specific sensory properties contribute towards the acceptance of a food.

This study aims to gain a better understanding of the consumer perceptions that underlie the negativity towards unusual novel foods by using consumer descriptive sensory profiling to investigate sensory perceptions before and after tasting products. The study also relates sensory perceptions to food appropriateness and willingness to eat to investigate how the experienced taste contributes to the acceptance of a novel food. Following the experimental design of previous work (Chapter 3 ), we randomize the presentation of labels and recipes of burger patties claimed to contain novel ingredients uncommonly eaten in the Netherlands (i.e. lamb brain, frog meat, mealworms). Due to the offensive nature of these novel foods, we used plant-based modifications of the burger patty recipes to create sensory differences between patties to prevent participants from ingesting these novel foods unknowingly as a result of the randomisation. While the absence of the actual ingredients does not allow conclusions to be drawn regarding the properties of the specific foods, it provides insights into how consumers perceive unusual novel foods. Outcomes from this study are targeted for the product development of culturally inappropriate foods like insects where the lack of appeal stands in the way of achieving consumer acceptance of a potentially good food.

\subsection{MATERIALS AND METHODS}

This study follows up previous work (Chapter 3) with a similar experimental design to further investigate the underlying sensory perceptions of unusual novel foods like insects. The current paper reports the results from 100 Dutch participants who performed descriptive sensory evaluations of beef-based burger patties (henceforth referred to as 'burger') claimed to contain beef or a partial substitution with unusual novel ingredients. We refer to the burger claimed to 
contain beef as the only meat ingredient as 'beef burger' and the burgers claimed to contain the unusual novel ingredients to be 'novel burgers'.

\subsubsection{Participants}

Participants were mainly Dutch students who have no food allergies and food restrictions and were recruited in the vicinity of the study location at Utrecht University, The Netherlands. In total, 100 participants ( 66 males, 34 females; $23.3 \pm 6.3$ years) completed the study. They were a different group of participants than those in the previous work (Chapter 3). Participants were not pre-informed that this study involved unusual foods like insects and were recruited on the basis of liking to eat beef burgers. This was done to avoid biasing the sample towards adventurous eaters. Food neophobia scores ranged from 11 to $54(M=25.5, S D=9.4$, Cronbach's $\alpha=0.84$ ), which is slightly less neophobic than the range of 11 to $63(M=30.1, S D=9.5)$ that was reported on a larger Dutch population (Schickenberg et al., 2008). Participants were all occasional or regular consumers of beef, whereas a minority claimed to have tasted the novel ingredients before [10 for lamb brain, 31 for frog meat, 25 for mealworms] but have never tasted them in the form of burger patties. During the session, participants were given the option not to eat the novel burgers without being penalized on their reward. Upon completion of the questionnaire, each participant received 7 euros.

\subsubsection{Labels, recipes and sensory attributes}

\subsubsection{Selection of unusual novel foods}

Unusual novel foods were selected based on a pre-test where 19 Dutch university students were asked to evaluate the familiarity, perceived appropriateness as food and expected sensoryliking of 12 uncommon foods. They were also asked to suggest words to describe the sensory experience of the novel foods when incorporated in a beef burger. Foods that participants did not know what it was (e.g. veal thymus), did not perceive to be suitable for making burgers (e.g. oysters), or did not expect to taste different from beef (e.g. horse) were not considered. Lamb brain, frog meat and mealworms were eventually selected for the study.

\subsubsection{Labels}

Labels presented with the burgers described only the meat composition, and participants were informed that the burgers to be tasted also contain other binding and seasoning ingredients that are not described in the label. The four labels presented were ' $100 \%$ ground beef', '75\% ground beef and $25 \%$ ground lamb brain', '75\% ground beef and $25 \%$ ground frog meat', and ' $75 \%$ ground beef and $25 \%$ ground mealworms'. For brevity, these labels will be mentioned as 'beef burger', 'lamb brain burger', 'frog meat burger' and 'mealworm burger' in this paper, with the latter three collectively described as 'novel burgers'. 


\subsubsection{Recipes}

Recipes were formulated by modifying a basic burger recipe consisting of ground beef, salt and pepper. Four burger recipe formulations were created with different amounts of breadcrumbs, mashed tofu and ground hazelnuts that were added to create perceivable texture and flavour differences while maintaining visual similarity between the burgers. This is to ensure that all recipes are credible when presented with all labels. The four recipe formulations (\% weight basis) are as follows recipe A: $94 \%$ beef, $2 \%$ breadcrumbs, $2 \%$ tofu, $2 \%$ hazelnuts; recipe B: $86 \%$ beef, $10 \%$ breadcrumbs, $2 \%$ tofu, $2 \%$ hazelnuts; recipe C: $80 \%$ beef, $2 \%$ breadcrumbs, $16 \%$ tofu, $2 \%$ hazelnuts; and recipe D: $88 \%$ beef, $2 \%$ breadcrumbs, $2 \%$ tofu, $8 \%$ hazelnuts. To each recipe, $0.5 \%$ salt and $0.4 \%$ ground black pepper were added. To make the burgers, the ingredients were mixed together, and separated into $50 \mathrm{~g}$ patties, then cooked on medium heat on a frying pan for about 10-12 min by flipping over every 1-2 min until cooked through.

\subsubsection{Selection of sensory descriptors}

A pre-test was performed with a different group of 12 Dutch students who were asked to describe the imagined taste, texture and appearance when the novel ingredients are incorporated into a beef burger. The most frequently mentioned expected sensory properties were meaty and juicy or tender for a beef burger, soft and slimy for a lamb brain burger, less beef-like or more chickenlike and tough or chewy for a frog meat burger, and nutty and grainy for a mealworm burger. Based on these descriptors, a preliminary list of 20 sensory attributes (10 flavour and 10 texture) were compiled for a pilot test with 6 other Dutch students who evaluated the burgers before and after tasting, as well as the ingredients using Rate-All-That-Applies (RATA) checklists. Based on the outcomes, 20 sensory attributes that were relevant and easy to understand were selected for the study. Flavour attributes included meaty, salty, nutty, fatty, mealy, musty, roasted, bitter, bland, and chicken, and texture attributes included juicy, tender, firm, dry, crunchy, slimy, grainy, fibrous, tough, and mushy.

\subsubsection{Procedures and questionnaire design}

\subsubsection{Experimental procedures}

Samples were cooked and packed into aluminium boxes in the week prior to the sensory sessions and stored at $-18^{\circ} \mathrm{C}$. Before the start of each session, frozen burgers were defrosted in a microwave, then heated in an oven at $200^{\circ} \mathrm{C}$ for $5 \mathrm{~min}$, and kept warm in heated porcelain dishes until served. Samples were served within 20 min of reheating. Sessions were conducted over two weeks between $10.30-20.00 \mathrm{~h}$ in a living room laboratory in the psychology department of Utrecht University, The Netherlands. Partitions were placed on the table to prevent communication between participants. An ethics committee of Wageningen University granted 
ethical approval, and participants filled in a consent form before beginning the session that lasted between 30-45 min.

\subsubsection{Questionnaire design}

The Dutch questionnaire that was used in this study was first designed in English and translated into Dutch by a native speaker. Accuracy of the translations were checked using back translations by another Dutch speaker. The questionnaire was structured in 4 parts with evaluations of: (1) beef burger (before and after tasting), (2) novel burgers (before tasting), (3) novel burgers (after tasting), and (4) willingness to eat burgers, and ingredient and participant measures. Presentation order of the novel burgers were randomized across questionnaires to control for contrast effects, but fixed within each questionnaire, i.e. a participant who evaluates frog meat burger followed by mealworm burger then lamb brain burger will receive that order throughout the questionnaire. Participants were randomly assigned to receive one of four possible sets of label-recipe combinations. A different recipe was served with each label, where each participant evaluated every label once and tasted every recipe once in a specific set of combinations. In total, 16 labelrecipe combinations ( 4 labels $\times 4$ recipes) were evaluated ( 4 combinations per participant).

In part 1, participants were presented an image of a burger patty, asked to imagine how a beef burger patty made with $100 \%$ beef would taste like, and to evaluate the expected sensory properties using Rate-All-That-Applies (RATA) checklists. This method has been reliably used for descriptive sensory profiling of various foods by untrained consumers (Ares et al., 2014; Oppermann, de Graaf, Scholten, Stieger, \& Piqueras-Fiszman, 2017). For the attribute checklists, sensory attributes were grouped by the 10 flavour and 10 texture attributes, and randomized within the respective tables to control for attention effects. Participants were instructed to first check the attributes that are applicable, then rate the intensity of the checked attributes on a 5-pt Likert scale anchored from low to high. An example using chicken burger was provided to demonstrate to participants how to use the RATA checklist, and participants were invited to clarify the meaning of terms they do not understand. After filling in the expected sensory profile, participants were served their assigned recipe for the beef burger label on a tray labelled with a random 3 digit code that was also printed on their questionnaire.

In part 2, participants were informed that they will be evaluating their expectations of unusual novel burgers. They were asked to imagine how each novel burger would taste like and fill in the RATA checklist as was done in the previous part. They were then asked how appropriate beef and novel burgers are as food (i.e. food appropriateness), which were evaluated on 9-pt Likert scales anchored from 'extremely inappropriate' to 'extremely appropriate'. Food appropriateness of beef burger was measured together with the novel burgers on the same page, such that participants can evaluate the novel burgers in relation to the beef burger. Hence, there was no measure of food appropriateness of beef burger before it was tasted. 
In part 3, participants were informed that they will be presented the novel burgers to taste, which they could either choose to taste and evaluate the experienced sensory properties, or to check a box that they had not tasted it and proceed with the next question. Participants were served their assigned recipe for each novel burger label. After completing the sensory evaluations, food appropriateness of all the burgers was measured again.

In part 4, future willingness to eat the burgers and ingredients in 3 situations (meal, special occasion, and snack) was measured on 5-pt Likert scales anchored from 'low' to 'high'. Participants were then asked to imagine the taste of the 4 ingredients (beef, lamb brain, frog meat, mealworms) when cooked but not incorporated into burger patties, and evaluate the expected sensory properties. Ingredient evaluations were performed in part 4, only after the burgers have been evaluated, in order to avoid a potential bias on burger evaluations. Food appropriateness of the ingredients was also measured, and familiarity with the ingredients was reported using 5 categories (1-not known as food, 2- known as food but never tasted, 3-tasted before, 4-eat occasionally, 5-eat regularly) according to Tuorila et al. (2001), and food neophobia scores were measured according to Pliner and Hobden (1992). Age, gender, and reasons for not tasting rejected novel burgers were then reported. Upon completing the questionnaire, participants were given a debriefing session during which they were informed that the novel burgers do not actually contain the novel ingredients, and that they should not tell others about the study until the end of the test period.

\subsubsection{Data analysis}

RATA ratings were transformed into scores (0-5), where 0 was when an attribute was not checked and 1-5 were the intensity scores for the checked attributes. Ratings of food appropriateness and willingness to eat were transformed into numbers: 1-9 (food appropriateness) and 1-5 (willingness to eat). Categories of familiarity were converted into a binary score where 1=tasted before and $0=$ never tasted. Ratings of the food neophobia scale (FNS) were treated according to Pliner and Hobden (1992), where scores were reversed on items 1, 4, 6, 9 and 10, and the sum of scores reported.

Repeated measures analysis of variance (ANOVA) was used to analyse the differences between attribute intensities for the expected sensory profiles of burgers and of ingredients. Principal component analysis on a correlational matrix was performed with the mean intensity ratings for burger and ingredient expectations to visualize the expected sensory differences. Correlational bi-plots were used to visualize the correlations between attributes before and after tasting. Differences in attribute intensities for individuals who have tasted and never tasted the novel ingredients were tested with independent samples t-tests. Two-way analysis of variance (ANOVA) was used to analyse the main and interaction effects of the labels and recipes on attribute intensities after tasting the burgers, and the differences in attribute intensities between each label and each recipe were analysed. Missing values due to the rejection of novel burgers 
were excluded on a case analysis basis. The relationships between sensory perceptions, food appropriateness, and willingness to eat were analysed by Pearson's product moment correlations. Multiple linear regressions were also performed to explore the influence of sensory perceptions on food appropriateness and willingness to eat.

\subsection{RESULTS}

\subsubsection{Investigating sensory perceptions before tasting}

\subsubsection{Expected sensory profiles of ingredients and burgers}

According to Figure 4.1 and Table 4.1, beef was expected to be more meaty, fatty, roasted, juicy, tender and firm, lamb brain was expected to be more fatty, musty, bitter, slimy and mushy, frog meat was expected to be more bitter, chicken, and tough, and mealworms were expected to be more nutty, mealy, musty, bitter, bland, dry, fibrous, grainy, crunchy and mushy. The expected sensory differences between the 4 ingredients were reduced when incorporated in burgers, as shown by the smaller effect sizes for all 20 attributes in Table 4.1. While the expected sensory profiles of beef and beef burger were very similar, those of the novel burgers appear to be a combination of beef and novel ingredient attributes (Figure 4.1). Many of these novel ingredient attributes are rather negative in valence (e.g. bitter, slimy), which may have a negative impact on the expected hedonic value. Presenting the novel ingredients within a burger patty reduced the expected intensities of

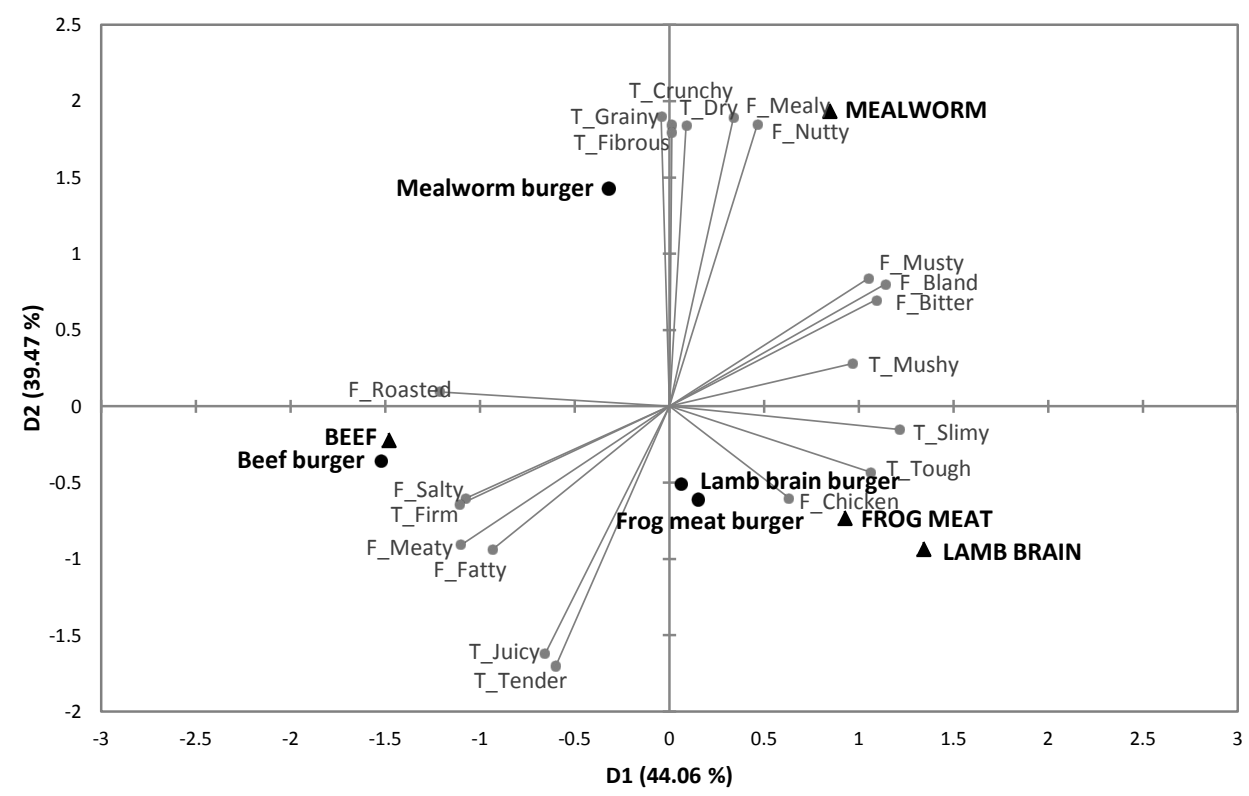

Figure 4.1. Principal component analysis based on the expected burger and ingredient attribute intensities. Flavour attributes are denoted by $F_{-}$and texture attributes denoted by $T_{-}$. 
these negative attributes, which might account for why familiar preparations help to improve the willingness to eat novel foods. Despite the unfamiliarity with the novel ingredients, the variance in attribute intensities did not appear any higher than that for evaluations of beef and beef burger (Table 4.1). An exception is for the attributes not typically associated with beef and beef burger (nutty, mealy, musty, bitter, chicken, slimy, mushy), where participants were very consistent (i.e. low variance) in finding them not applicable to beef and beef burger.

Table 4.1. Mean attribute intensities with standard deviations for the expected sensory profiles of burgers and ingredients.

\begin{tabular}{|c|c|c|c|c|c|c|c|c|c|c|c|c|}
\hline & \multicolumn{6}{|c|}{ Burger expectations $(n=100)$} & \multicolumn{6}{|c|}{ Ingredient expectations $(n=100)$} \\
\hline & Beef & $\begin{array}{l}\text { Lamb } \\
\text { brain }\end{array}$ & $\begin{array}{l}\text { Frog } \\
\text { meat }\end{array}$ & $\begin{array}{l}\text { Meal } \\
\text { worm }\end{array}$ & $\mathrm{p}$ & $\eta_{p}^{2}$ & Beef & $\begin{array}{l}\text { Lamb } \\
\text { brain }\end{array}$ & $\begin{array}{l}\text { Frog } \\
\text { meat }\end{array}$ & $\begin{array}{l}\text { Meal } \\
\text { worm }\end{array}$ & $\mathrm{p}$ & $\eta_{p}^{2}$ \\
\hline \multicolumn{13}{|l|}{ Flavour } \\
\hline Meaty & $\begin{array}{l}4.0^{\mathrm{a}} \\
(1.3)\end{array}$ & $\begin{array}{l}3.4^{\mathrm{b}} \\
(1.4)\end{array}$ & $\begin{array}{l}3.2^{b} \\
(1.6)\end{array}$ & $\begin{array}{l}2.7^{c} \\
(1.7)\end{array}$ & $* * *$ & 0.22 & $\begin{array}{l}4.4^{\mathrm{a}} \\
(1.1)\end{array}$ & $\begin{array}{l}2.0^{\mathrm{b}} \\
(1.8)\end{array}$ & $\begin{array}{l}1.9^{\mathrm{b}} \\
(1.8)\end{array}$ & $\begin{array}{l}0.8^{c} \\
(1.4)\end{array}$ & $* * *$ & 0.54 \\
\hline Salty & $\begin{array}{l}2.5^{\mathrm{a}} \\
(0.5)\end{array}$ & $\begin{array}{l}2.2^{\mathrm{a}} \\
(1.7)\end{array}$ & $\begin{array}{l}2.0^{\mathrm{a}} \\
(1.7)\end{array}$ & $\begin{array}{l}2.0^{\mathrm{a}} \\
(1.7)\end{array}$ & $*$ & 0.03 & $\begin{array}{l}2.3^{\mathrm{a}} \\
(1.6)\end{array}$ & $\begin{array}{l}1.4^{b} \\
(1.6)\end{array}$ & $\begin{array}{l}1.1^{b} \\
(1.5)\end{array}$ & $\begin{array}{l}0.9^{b} \\
(1.4)\end{array}$ & $* * *$ & 0.19 \\
\hline Nutty & $\begin{array}{l}0.1^{b} \\
(0.5)\end{array}$ & $\begin{array}{l}0.3^{\mathrm{b}} \\
(1.0)\end{array}$ & $\begin{array}{l}0.2^{b} \\
(0.8)\end{array}$ & $\begin{array}{l}1.1^{\mathrm{a}} \\
(1.7)\end{array}$ & $* * *$ & 0.19 & $\begin{array}{l}0.2^{b} \\
(0.7)\end{array}$ & $\begin{array}{l}0.4^{\mathrm{b}} \\
(1.1)\end{array}$ & $\begin{array}{l}0.4^{b} \\
(1.1)\end{array}$ & $\begin{array}{l}1.5^{\mathrm{a}} \\
(1.9)\end{array}$ & $* * *$ & 0.20 \\
\hline Fatty & $\begin{array}{l}3.0^{a} \\
(1.4)\end{array}$ & $\begin{array}{l}2.5^{a} \\
(1.7)\end{array}$ & $\begin{array}{l}1.8^{6} \\
(1.6)\end{array}$ & $\begin{array}{l}1.6^{6} \\
(1.6)\end{array}$ & $* * *$ & 0.20 & $\begin{array}{l}2.4^{a} \\
(1.5)\end{array}$ & $\begin{array}{l}1.8^{\mathrm{a}} \\
(1.8)\end{array}$ & $\begin{array}{l}0.9^{b} \\
(1.4)\end{array}$ & $\begin{array}{c}0.7 \\
(1.3)\end{array}$ & $* * *$ & 0.24 \\
\hline Mealy & $\begin{array}{l}0.2^{b} \\
(0.6)\end{array}$ & $\begin{array}{l}0.5^{b} \\
\text { (1.1) }\end{array}$ & $\begin{array}{l}0.3^{6} \\
(0.9)\end{array}$ & $\begin{array}{l}2.0^{\mathrm{a}} \\
(1.9)\end{array}$ & $* * *$ & 0.37 & $\begin{array}{l}0.1^{b} \\
(0.6)\end{array}$ & $\begin{array}{l}0.4^{b} \\
(1.1)\end{array}$ & $\begin{array}{l}0.2^{b} \\
(0.8)\end{array}$ & $\begin{array}{l}2.4^{\mathrm{a}} \\
(1.9)\end{array}$ & $* * *$ & 0.46 \\
\hline Musty & $\begin{array}{l}0.3^{\mathrm{c}} \\
(0.8)\end{array}$ & $\begin{array}{l}1.0^{\mathrm{ab}} \\
(1.4)\end{array}$ & $\begin{array}{l}0.6^{\mathrm{bc}} \\
(1.3)\end{array}$ & $\begin{array}{l}1.2^{\mathrm{a}} \\
(1.7)\end{array}$ & $* * *$ & 0.10 & $\begin{array}{l}0.2^{\mathrm{c}} \\
(0.7)\end{array}$ & $\begin{array}{l}1.6^{\mathrm{a}} \\
(1.9)\end{array}$ & $\begin{array}{l}0.8^{\mathrm{b}} \\
(1.4)\end{array}$ & $\begin{array}{l}1.7^{\mathrm{a}} \\
(1.8)\end{array}$ & $* * *$ & 0.21 \\
\hline Bitter & $\begin{array}{l}0.2^{b} \\
(0.6)\end{array}$ & $\begin{array}{l}0.7^{a} \\
(1.3)\end{array}$ & $\begin{array}{l}0.5^{a} \\
(1.2)\end{array}$ & $\begin{array}{l}0.5^{a} \\
(1.1)\end{array}$ & $* *$ & 0.05 & $\begin{array}{l}0.1^{b} \\
(0.4)\end{array}$ & $\begin{array}{l}0.7^{\mathrm{a}} \\
(1.4)\end{array}$ & $\begin{array}{l}0.5^{a} \\
(1.2)\end{array}$ & $\begin{array}{l}0.9^{a} \\
(1.5)\end{array}$ & $* * *$ & 0.08 \\
\hline Roasted & $\begin{array}{l}2.7^{\mathrm{a}} \\
(1.5)\end{array}$ & $\begin{array}{l}1.3^{6} \\
(1.5)\end{array}$ & $\begin{array}{l}1.5^{b} \\
(1.6)\end{array}$ & $\begin{array}{l}1.5^{b} \\
(1.7)\end{array}$ & $* * *$ & 0.22 & $\begin{array}{l}1.9^{\mathrm{a}} \\
(1.8)\end{array}$ & $\begin{array}{l}0.2^{\mathrm{C}} \\
(0.8)\end{array}$ & $\begin{array}{l}0.8^{6} \\
(1.5)\end{array}$ & $\begin{array}{l}1.0^{\mathrm{b}} \\
(1.6)\end{array}$ & $* * *$ & 0.25 \\
\hline Bland & $\begin{array}{l}0.5^{b} \\
(1.2)\end{array}$ & $\begin{array}{l}0.9^{\mathrm{ab}} \\
(1.5)\end{array}$ & $\begin{array}{l}1.0^{\mathrm{a}} \\
(1.5)\end{array}$ & $\begin{array}{l}1.0^{\mathrm{a}} \\
(1.6)\end{array}$ & $*$ & 0.03 & $\begin{array}{l}0.5^{\mathrm{c}} \\
(1.1)\end{array}$ & $\begin{array}{l}1.5^{\mathrm{ab}} \\
(1.8)\end{array}$ & $\begin{array}{l}1.2^{b} \\
(1.6)\end{array}$ & $\begin{array}{l}1.8^{\mathrm{a}} \\
(2.0)\end{array}$ & $* * *$ & 0.14 \\
\hline Chicken & $\begin{array}{l}0.0^{6} \\
(0.2)\end{array}$ & $\begin{array}{l}0.1^{b} \\
(0.6)\end{array}$ & $\begin{array}{l}1.3^{a} \\
(1.6)\end{array}$ & $\begin{array}{l}0.2^{b} \\
(0.7)\end{array}$ & $* * *$ & 0.29 & $\begin{array}{l}0.0^{c} \\
(0.0)\end{array}$ & $\begin{array}{l}0.3^{\mathrm{b}} \\
(1.0)\end{array}$ & $\begin{array}{l}2.2^{a} \\
(1.9)\end{array}$ & $\begin{array}{l}0.4^{b} \\
(1.0)\end{array}$ & $* * *$ & 0.42 \\
\hline Texture & & & & & & & & & & & & \\
\hline Juicy & $\begin{array}{l}2.6^{a} \\
(1.6)\end{array}$ & $\begin{array}{l}2.5^{\mathrm{a}} \\
(1.8)\end{array}$ & $\begin{array}{l}2.1^{\mathrm{a}} \\
(1.8)\end{array}$ & $\begin{array}{l}1.4^{b} \\
(1.6)\end{array}$ & $* * *$ & 0.13 & $\begin{array}{l}2.7^{\mathrm{a}} \\
(1.8)\end{array}$ & $\begin{array}{l}2.3^{\mathrm{ab}} \\
(1.9)\end{array}$ & $\begin{array}{l}1.9^{\mathrm{b}} \\
(1.8)\end{array}$ & $\begin{array}{l}1.0^{c} \\
(1.7)\end{array}$ & $* * *$ & 0.17 \\
\hline Tender & $\begin{array}{l}2.4^{\mathrm{a}} \\
(1.7)\end{array}$ & $\begin{array}{l}2.3^{\mathrm{a}} \\
(1.9)\end{array}$ & $\begin{array}{l}2.1^{\mathrm{a}} \\
(1.8)\end{array}$ & $\begin{array}{l}1.2^{b} \\
(1.6)\end{array}$ & $* * *$ & 0.12 & $\begin{array}{l}2.8^{\mathrm{a}} \\
(1.8)\end{array}$ & $\begin{array}{l}2.1^{\mathrm{b}} \\
(1.8)\end{array}$ & $\begin{array}{l}2.2^{\mathrm{ab}} \\
(2.0)\end{array}$ & $\begin{array}{c}0.7^{c} \\
(1.3)\end{array}$ & $* * *$ & 0.22 \\
\hline Firm & $\begin{array}{l}2.7^{\mathrm{a}} \\
(1.8)\end{array}$ & $\begin{array}{l}1.6^{b} \\
\text { (1.7) }\end{array}$ & $\begin{array}{l}1.8^{\mathrm{b}} \\
\text { (1.9) }\end{array}$ & $\begin{array}{l}1.7^{b} \\
(1.8)\end{array}$ & $* * *$ & 0.11 & $\begin{array}{l}2.8^{\mathrm{a}} \\
(1.6)\end{array}$ & $\begin{array}{l}1.3^{\mathrm{C}} \\
(1.8)\end{array}$ & $\begin{array}{l}2.0^{b} \\
(1.9)\end{array}$ & $\begin{array}{l}1.1^{\mathrm{c}} \\
(1.6)\end{array}$ & $* * *$ & 0.18 \\
\hline Dry & $\begin{array}{l}1.2^{\mathrm{b}} \\
(1.6)\end{array}$ & $\begin{array}{l}1.0^{\mathrm{b}} \\
(1.4)\end{array}$ & $\begin{array}{l}1.1^{\mathrm{b}} \\
(1.4)\end{array}$ & $\begin{array}{l}2.2^{\mathrm{a}} \\
(1.8)\end{array}$ & $* * *$ & 0.14 & $\begin{array}{l}1.2^{b c} \\
(1.6)\end{array}$ & $\begin{array}{c}0.8^{\mathrm{c}} \\
(1.4)\end{array}$ & $\begin{array}{l}1.3^{\mathrm{b}} \\
(1.7)\end{array}$ & $\begin{array}{l}2.0^{\mathrm{a}} \\
(2.0)\end{array}$ & $* * *$ & 0.29 \\
\hline Fibrous & $\begin{array}{l}0.6^{b} \\
(1.3)\end{array}$ & $\begin{array}{l}0.8^{\mathrm{ab}} \\
(1.5)\end{array}$ & $\begin{array}{l}0.8^{\mathrm{ab}} \\
(1.4)\end{array}$ & $\begin{array}{l}1.2^{\mathrm{a}} \\
(1.7)\end{array}$ & $*$ & 0.03 & $\begin{array}{l}1.0^{\mathrm{ab}} \\
(1.6)\end{array}$ & $\begin{array}{l}0.7^{b} \\
(1.4)\end{array}$ & $\begin{array}{l}0.8^{b} \\
(1.4)\end{array}$ & $\begin{array}{l}1.4^{\mathrm{a}} \\
(1.8)\end{array}$ & $* *$ & 0.04 \\
\hline Slimy & $\begin{array}{l}0.1^{c} \\
(0.4)\end{array}$ & $\begin{array}{l}1.2^{\mathrm{ab}} \\
(1.6)\end{array}$ & $\begin{array}{l}1.5^{\mathrm{a}} \\
(1.7)\end{array}$ & $\begin{array}{l}0.8^{b} \\
(1.5)\end{array}$ & $* * *$ & 0.20 & $\begin{array}{l}0.1^{\mathrm{c}} \\
(0.3)\end{array}$ & $\begin{array}{l}2.0^{\mathrm{a}} \\
(2.0)\end{array}$ & $\begin{array}{l}1.2^{\mathrm{b}} \\
(1.7)\end{array}$ & $\begin{array}{l}1.5^{\mathrm{ab}} \\
(2.0)\end{array}$ & $* * *$ & 0.22 \\
\hline Grainy & $\begin{array}{l}0.6^{b} \\
(1.2)\end{array}$ & $\begin{array}{l}0.9^{b} \\
(1.5)\end{array}$ & $\begin{array}{l}0.6^{6} \\
(1.3)\end{array}$ & $\begin{array}{l}1.9^{\mathrm{a}} \\
(1.8)\end{array}$ & $* * *$ & 0.18 & $\begin{array}{l}0.8^{b} \\
(1.4)\end{array}$ & $\begin{array}{l}0.4^{b c} \\
(1.2)\end{array}$ & $\begin{array}{l}0.3^{\mathrm{c}} \\
(0.9)\end{array}$ & $\begin{array}{l}1.8^{\mathrm{a}} \\
(2.0)\end{array}$ & $* * *$ & 0.20 \\
\hline Crunchy & $\begin{array}{l}0.7^{\mathrm{ab}} \\
(1.2)\end{array}$ & $\begin{array}{l}0.4^{\mathrm{b}} \\
(1.1)\end{array}$ & $\begin{array}{l}0.6^{\mathrm{ab}} \\
(1.3)\end{array}$ & $\begin{array}{l}0.9^{\mathrm{a}} \\
(1.5)\end{array}$ & $* *$ & 0.04 & $\begin{array}{l}0.6^{b} \\
(1.3)\end{array}$ & $\begin{array}{l}0.2^{\mathrm{C}} \\
(0.7)\end{array}$ & $\begin{array}{l}0.6^{b} \\
(1.4)\end{array}$ & $\begin{array}{l}1.6^{\mathrm{a}} \\
(2.0)\end{array}$ & $* * *$ & 0.17 \\
\hline Tough & $\begin{array}{l}0.7^{b} \\
(1.2)\end{array}$ & $\begin{array}{l}1.2^{\mathrm{ab}} \\
(1.7)\end{array}$ & $\begin{array}{l}1.3^{\mathrm{a}} \\
(1.6)\end{array}$ & $\begin{array}{l}1.1^{\mathrm{ab}} \\
(1.6)\end{array}$ & $*$ & 0.03 & $\begin{array}{l}0.7^{c} \\
(1.2)\end{array}$ & $\begin{array}{l}1.4^{\mathrm{ab}} \\
(1.8)\end{array}$ & $\begin{array}{l}2.0^{\mathrm{a}} \\
(1.8)\end{array}$ & $\begin{array}{l}1.2^{b c} \\
(1.7)\end{array}$ & $* * *$ & 0.11 \\
\hline Mushy & $\begin{array}{l}0.1^{b} \\
(0.5)\end{array}$ & $\begin{array}{l}1.2^{\mathrm{a}} \\
(1.6)\end{array}$ & $\begin{array}{l}0.8^{\mathrm{a}} \\
(1.4)\end{array}$ & $\begin{array}{l}1.0^{\mathrm{a}} \\
(1.5)\end{array}$ & $* * *$ & 0.13 & $\begin{array}{l}0.0^{c} \\
(0.2)\end{array}$ & $\begin{array}{l}1.7^{\mathrm{a}} \\
(1.9)\end{array}$ & $\begin{array}{l}0.4^{b} \\
(1.2)\end{array}$ & $\begin{array}{l}1.1^{\mathrm{a}} \\
(1.8)\end{array}$ & $* * *$ & 0.22 \\
\hline
\end{tabular}

Repeated measures one-way ANOVA's performed to analyse differences between burgers and ingredients. Standard deviations are indicated in italics. Bold highlights indicate significant values. Significance levels indicated by $*_{p}<0.05, * * p<0.01, * * * p<0.001$, and n.s. as non-significant $(p>0.05)$. Different letters denote significant differences between means of burgers and of ingredients. 


\subsubsection{Effect of prior taste experience on expected sensory properties}

The expected sensory profiles of the novel ingredients differed significantly between participants who have and have not tasted it before (Figure 4.2).
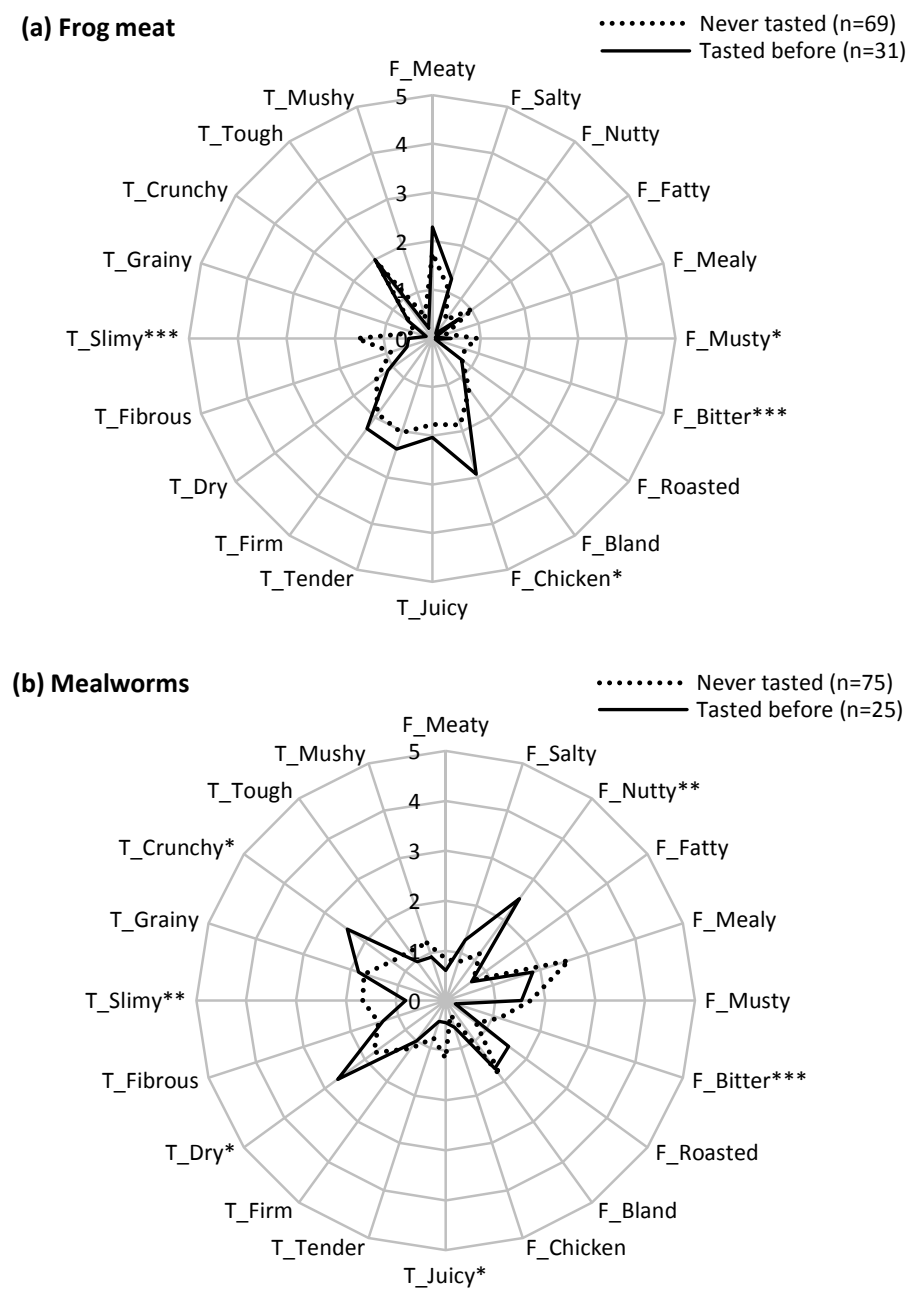

Figure 4.2. Mean attribute intensities for the expected sensory profile of (a) frog meat and (b) mealworms by participants who have never tasted (dotted lines) and have tasted (solid lines) the ingredients before. Independent samples t-tests performed on each attribute to test for differences between means, and significance levels are indicated by ${ }^{*} \mathrm{p}<0.05,{ }^{* *} \mathrm{p}<0.01, * * * \mathrm{p}<0.001$.

Only the expected sensory profiles of frog meat (Figure $\mathbf{2 a}$ ) and mealworms (Figure $\mathbf{2 b}$ ) were compared as very few participants $(n=10)$ claimed to have tasted lamb brains before, of which several were uncertain about it. Participants who have tasted frog meat before $(n=31)$ expected frog meat to taste more chicken than those who have never tasted it before $(n=69)$, whereas 
those who have never tasted it before expected it to be more slimy, bitter and musty. Participants who have tasted mealworms before $(n=25)$ expected mealworms to taste more nutty, crunchy and dry than those who have never tasted it before $(n=75)$, whereas those who have never tasted it before expected it to be more slimy, bitter and juicy. Participants who have never tasted the novel ingredients before seemed to associate more negative attributes than those who have tasted them before.

\subsubsection{Investigating sensory perceptions after tasting}

\subsubsection{Experienced sensory profiles of burgers}

Of the 100 participants, 5 rejected the lamb brain burger and 6 rejected the mealworm burger (i.e. they did not taste the burger at all). These missing values were excluded from analyses on a case-wise basis. The main effects of labels and recipes on attribute intensities after tasting are shown in Table 4.2. Labels had a small but significant effect on 4 of the 20 attributes (meaty, mealy, chicken, dry). Samples were evaluated as more meaty and dry when labelled as a beef burger, more chicken when labelled as a frog meat burger, and more mealy when labelled as a mealworm burger. The recipe tasted had significant effects on 14 of the 20 attributes (meaty, salty, nutty, fatty, mealy, musty, juicy, tender, firm, dry, slimy, grainy), and effect sizes were also generally larger. In comparison to the other recipes tasted, recipe A was evaluated to be more meaty, salty, firm, dry and tough, recipe B was more fatty, mealy, juicy, tender, slimy and mushy, recipe $C$ was more mealy, firm, dry and tough, and recipe D was more nutty, mealy, musty, firm, $d r y$, grainy, and tough. Overall, in comparison to recipe A, the additional breadcrumbs in recipe $B$ contributed to a softer texture, and the additional hazelnuts in recipe $D$ contributed to a more nutty and less meaty flavour, whereas the additional tofu in recipe $C$ resulted in a slightly less meaty flavour without much change in texture.

There was also a significant label-recipe interaction effect for 2 of the 20 attributes (nutty, tough). The interaction effect on the attributes nutty $\left[p=0.012, \eta_{p}{ }^{2}=0.05\right]$ and tough $[p=0.008$, $\left.\eta_{p}{ }^{2}=0.06\right]$ indicates that the label presented had influenced the way in which the experienced sensory properties were evaluated. Figure $\mathbf{4 . 3}$ shows the mean intensities of these attributes according to recipe and label. Recipe D (additional hazelnuts) was evaluated to be more nutty when presented with the novel burger labels but not when presented with the beef burger label (Figure 4.3a). This suggests that participants were less likely to detect or identify the nutty taste when they seemed quite certain in their expectations that the beef burger would not taste nutty (see Table 4.1). In the case of the attribute tough (Figure 4.3b), recipes A, C and D differed in how tough they were perceived to be when presented with different labels, whereas recipe $B$ with the softer texture was consistently perceived to be less tough than the other recipes. Overall, these results indicate that the recipe tasted had a much stronger influence on the sensory perceptions after tasting than the label presented, but the label also influences how the experienced sensory properties were evaluated. 
Table 4.2. Mean attribute intensities with standard deviations for the experienced burgers according to label and to recipe.

\begin{tabular}{|c|c|c|c|c|c|c|c|c|c|c|c|c|}
\hline & \multicolumn{6}{|c|}{ Experienced burgers: Label effects } & \multicolumn{6}{|c|}{ Experienced burgers: Recipe effects } \\
\hline & $\begin{array}{c}\text { Beef } \\
n=100\end{array}$ & $\begin{array}{l}\text { Lamb } \\
\text { brain } \\
\mathrm{n}=95\end{array}$ & $\begin{array}{l}\text { Frog } \\
\text { meat } \\
n=100\end{array}$ & $\begin{array}{c}\text { Meal } \\
\text { worm } \\
n=94\end{array}$ & $\mathrm{p}$ & $\eta_{p}^{2}$ & $\begin{array}{c}\text { Rec. } \\
\text { A } \\
n=99\end{array}$ & $\begin{array}{c}\text { Rec. } \\
\text { B } \\
n=95\end{array}$ & $\begin{array}{c}\text { Rec. } \\
\text { C } \\
n=98\end{array}$ & $\begin{array}{c}\text { Rec. } \\
D \\
n=97\end{array}$ & $\mathrm{p}$ & $\eta_{p}^{2}$ \\
\hline \multicolumn{13}{|l|}{ Flavour } \\
\hline Meaty & $\begin{array}{l}3.6^{\mathrm{a}} \\
(1.4)\end{array}$ & $\begin{array}{l}3.2^{\mathrm{ab}} \\
(1.6)\end{array}$ & $\begin{array}{l}3.0^{\mathrm{b}} \\
(1.7)\end{array}$ & $\begin{array}{l}3.0^{\mathrm{ab}} \\
(1.6)\end{array}$ & $*$ & 0.02 & $\begin{array}{l}3.6^{\mathrm{a}} \\
(1.4)\end{array}$ & $\begin{array}{l}3.2^{\mathrm{ab}} \\
(1.7)\end{array}$ & $\begin{array}{l}3.2^{\mathrm{ab}} \\
(1.6)\end{array}$ & $\begin{array}{l}2.9^{b} \\
(1.6)\end{array}$ & $*$ & 0.03 \\
\hline Salty & $\begin{array}{c}2.4 \\
(1.5)\end{array}$ & $\begin{array}{c}2.1 \\
(1.6)\end{array}$ & $\begin{array}{c}2.1 \\
(1.7)\end{array}$ & $\begin{array}{c}2.0 \\
(1.6)\end{array}$ & n.s. & 0.01 & $\begin{array}{l}2.4^{\mathrm{a}} \\
(1.7)\end{array}$ & $\begin{array}{l}1.9^{\mathrm{ab}} \\
(1.5)\end{array}$ & $\begin{array}{l}2.3^{\mathrm{ab}} \\
(1.6)\end{array}$ & $\begin{array}{l}1.8^{\mathrm{b}} \\
(1.6)\end{array}$ & $* *$ & 0.03 \\
\hline Nutty & $\begin{array}{c}0.5 \\
(1.2)\end{array}$ & $\begin{array}{c}0.6 \\
(1.4)\end{array}$ & $\begin{array}{c}0.7 \\
(1.5)\end{array}$ & $\begin{array}{c}1.0 \\
(1.6)\end{array}$ & n.s. & 0.02 & $\begin{array}{l}0.4^{b} \\
(1.1)\end{array}$ & $\begin{array}{l}0.4^{b} \\
(1.1)\end{array}$ & $\begin{array}{l}0.4^{b} \\
(1.1)\end{array}$ & $\begin{array}{l}1.6^{\mathrm{a}} \\
(2.0)\end{array}$ & $* * *$ & 0.13 \\
\hline Fatty & $\begin{array}{c}1.7 \\
(1.5)\end{array}$ & $\begin{array}{c}1.8 \\
(1.7)\end{array}$ & $\begin{array}{c}1.6 \\
(1.6)\end{array}$ & $\begin{array}{c}1.6 \\
(1.5)\end{array}$ & n.s. & 0.00 & $\begin{array}{l}1.5^{b} \\
(1.4)\end{array}$ & $\begin{array}{l}2.4^{a} \\
(1.7)\end{array}$ & $\begin{array}{l}1.4^{b} \\
(1.5)\end{array}$ & $\begin{array}{l}1.4^{6} \\
(1.5)\end{array}$ & $* * *$ & 0.07 \\
\hline Mealy & $\begin{array}{l}0.7^{b} \\
(1.3)\end{array}$ & $\begin{array}{l}0.8^{\mathrm{ab}} \\
(1.3)\end{array}$ & $\begin{array}{l}0.8^{\mathrm{ab}} \\
(1.4)\end{array}$ & $\begin{array}{l}1.3^{\mathrm{a}} \\
(1.7)\end{array}$ & $*$ & 0.03 & $\begin{array}{l}0.4^{b} \\
(1.0)\end{array}$ & $\begin{array}{l}1.0^{\mathrm{a}} \\
(1.5)\end{array}$ & $\begin{array}{l}0.9^{\mathrm{a}} \\
(1.4)\end{array}$ & $\begin{array}{l}1.2^{\mathrm{a}} \\
(1.7)\end{array}$ & $* * *$ & 0.05 \\
\hline Musty & $\begin{array}{c}0.7 \\
(1.4)\end{array}$ & $\begin{array}{c}0.7 \\
(1.3)\end{array}$ & $\begin{array}{c}0.7 \\
(1.3)\end{array}$ & $\begin{array}{c}1.1 \\
(1.6)\end{array}$ & n.s. & 0.01 & $\begin{array}{l}0.6^{b} \\
(1.2)\end{array}$ & $\begin{array}{l}0.6^{\mathrm{ab}} \\
(1.3)\end{array}$ & $\begin{array}{l}0.8^{\mathrm{ab}} \\
(1.4)\end{array}$ & $\begin{array}{l}1.2^{a} \\
(1.6)\end{array}$ & $*$ & 0.03 \\
\hline Bitter & $\begin{array}{c}0.2 \\
(0.6)\end{array}$ & $\begin{array}{c}0.5 \\
(1.1)\end{array}$ & $\begin{array}{c}0.4 \\
(1.0)\end{array}$ & $\begin{array}{c}0.4 \\
(1.0)\end{array}$ & n.s. & 0.01 & $\begin{array}{c}0.5 \\
(1.2)\end{array}$ & $\begin{array}{c}0.2 \\
(0.8)\end{array}$ & $\begin{array}{c}0.3 \\
(0.9)\end{array}$ & $\begin{array}{c}0.2 \\
(0.8)\end{array}$ & n.s. & 0.02 \\
\hline Roasted & $\begin{array}{c}1.2 \\
(1.6)\end{array}$ & $\begin{array}{c}1.4 \\
(1.6)\end{array}$ & $\begin{array}{c}1.2 \\
(1.6)\end{array}$ & $\begin{array}{c}1.3 \\
(1.6)\end{array}$ & n.s. & 0.00 & $\begin{array}{c}1.5 \\
(1.7)\end{array}$ & $\begin{array}{c}1.2 \\
(1.5)\end{array}$ & $\begin{array}{c}1.4 \\
(1.6)\end{array}$ & $\begin{array}{c}1.2 \\
(1.6)\end{array}$ & n.s. & 0.01 \\
\hline Bland & $\begin{array}{c}1.2 \\
(1.6)\end{array}$ & $\begin{array}{c}0.8 \\
(1.4)\end{array}$ & $\begin{array}{c}1.1 \\
(1.6)\end{array}$ & $\begin{array}{c}1.0 \\
(1.6)\end{array}$ & n.s. & 0.01 & $\begin{array}{c}0.7 \\
(1.3)\end{array}$ & $\begin{array}{c}1.3 \\
(1.7)\end{array}$ & $\begin{array}{c}1.0 \\
(1.6)\end{array}$ & $\begin{array}{c}1.1 \\
(1.6)\end{array}$ & n.s. & 0.02 \\
\hline Chicken & $\begin{array}{l}0.1^{b} \\
(0.4)\end{array}$ & $\begin{array}{l}0.1^{b} \\
(0.3)\end{array}$ & $\begin{array}{l}0.4^{\mathrm{a}} \\
(1.0)\end{array}$ & $\begin{array}{l}0.1^{b} \\
(0.4)\end{array}$ & $* * *$ & 0.06 & $\begin{array}{c}0.1 \\
(0.5)\end{array}$ & $\begin{array}{c}0.1 \\
(0.4)\end{array}$ & $\begin{array}{c}0.3 \\
(0.9)\end{array}$ & $\begin{array}{c}0.1 \\
(0.6)\end{array}$ & n.s. & 0.02 \\
\hline \multicolumn{13}{|l|}{ Texture } \\
\hline Juicy & $\begin{array}{c}1.3 \\
(1.5)\end{array}$ & $\begin{array}{c}1.7 \\
(1.8)\end{array}$ & $\begin{array}{c}1.6 \\
(1.7)\end{array}$ & $\begin{array}{c}1.2 \\
(1.6)\end{array}$ & n.s. & 0.02 & $\begin{array}{l}1.3^{b} \\
(1.6)\end{array}$ & $\begin{array}{l}2.3^{\mathrm{a}} \\
(1.7)\end{array}$ & $\begin{array}{l}1.1^{b} \\
(1.5)\end{array}$ & $\begin{array}{l}1.1^{b} \\
(1.5)\end{array}$ & $* * *$ & 0.09 \\
\hline Tender & $\begin{array}{c}1.5 \\
(1.6)\end{array}$ & $\begin{array}{c}1.4 \\
(1.7)\end{array}$ & $\begin{array}{c}1.5 \\
(1.7)\end{array}$ & $\begin{array}{c}1.5 \\
(1.8)\end{array}$ & n.s. & 0.00 & $\begin{array}{l}1.3^{b} \\
(1.6)\end{array}$ & $\begin{array}{l}2.3^{\mathrm{a}} \\
(1.8)\end{array}$ & $\begin{array}{l}1.2^{b} \\
(1.6)\end{array}$ & $\begin{array}{l}1.0^{b} \\
(1.5)\end{array}$ & $* * *$ & 0.10 \\
\hline Firm & $\begin{array}{c}2.3 \\
(1.7)\end{array}$ & $\begin{array}{c}2.0 \\
(1.7)\end{array}$ & $\begin{array}{c}2.2 \\
(1.8)\end{array}$ & $\begin{array}{c}2.1 \\
(1.9)\end{array}$ & n.s. & 0.00 & $\begin{array}{c}2.8^{\mathrm{a}} \\
(1.6)\end{array}$ & $\begin{array}{l}1.3^{b} \\
(1.6)\end{array}$ & $\begin{array}{l}2.3^{a} \\
(1.8)\end{array}$ & $\begin{array}{l}2.2^{a} \\
(1.9)\end{array}$ & $* * *$ & 0.08 \\
\hline Dry & $\begin{array}{l}2.7^{a} \\
(1.7)\end{array}$ & $\begin{array}{l}1.7^{6} \\
(1.7)\end{array}$ & $\begin{array}{l}2.0^{a b} \\
(1.7)\end{array}$ & $\begin{array}{l}2.1^{\mathrm{ab}} \\
(1.8)\end{array}$ & $* * *$ & 0.04 & $\begin{array}{l}2.3^{\mathrm{a}} \\
(1.8)\end{array}$ & $\begin{array}{l}1.1^{b} \\
(1.5)\end{array}$ & $\begin{array}{l}2.5^{a} \\
(1.8)\end{array}$ & $\begin{array}{l}2.6^{a} \\
(1.6)\end{array}$ & $* * *$ & 0.12 \\
\hline Fibrous & $\begin{array}{c}1.2 \\
(1.7)\end{array}$ & $\begin{array}{c}1.1 \\
(1.6)\end{array}$ & $\begin{array}{c}1.2 \\
(1.6)\end{array}$ & $\begin{array}{c}1.2 \\
(1.7)\end{array}$ & n.s. & 0.00 & $\begin{array}{c}1.2 \\
(1.7)\end{array}$ & $\begin{array}{c}0.8 \\
(1.4)\end{array}$ & $\begin{array}{c}1.3 \\
(1.7)\end{array}$ & $\begin{array}{c}1.4 \\
(1.8)\end{array}$ & n.s. & 0.02 \\
\hline Slimy & $\begin{array}{c}0.3 \\
(1.0)\end{array}$ & $\begin{array}{c}0.5 \\
(1.2)\end{array}$ & $\begin{array}{c}0.4 \\
(0.9)\end{array}$ & $\begin{array}{c}0.5 \\
(1.1)\end{array}$ & n.s. & 0.01 & $\begin{array}{l}0.2^{b} \\
(0.6)\end{array}$ & $\begin{array}{l}0.9^{\mathrm{a}} \\
(1.5)\end{array}$ & $\begin{array}{l}0.3^{b} \\
(0.8)\end{array}$ & $\begin{array}{l}0.3^{b} \\
(0.9)\end{array}$ & $* * *$ & 0.08 \\
\hline Grainy & $\begin{array}{c}2.2 \\
(1.9)\end{array}$ & $\begin{array}{c}2.0 \\
(1.8)\end{array}$ & $\begin{array}{c}1.9 \\
(1.8)\end{array}$ & $\begin{array}{c}2.0 \\
(1.9)\end{array}$ & n.s. & 0.00 & $\begin{array}{l}1.9^{b} \\
(1.8)\end{array}$ & $\begin{array}{l}1.5^{b} \\
(1.7)\end{array}$ & $\begin{array}{l}2.1^{\mathrm{ab}} \\
(1.8)\end{array}$ & $\begin{array}{l}2.7^{a} \\
(1.8)\end{array}$ & $* * *$ & 0.06 \\
\hline Crunchy & $\begin{array}{c}0.4 \\
(1.0)\end{array}$ & $\begin{array}{c}0.4 \\
(1.0)\end{array}$ & $\begin{array}{c}0.4 \\
(1.1)\end{array}$ & $\begin{array}{c}0.5 \\
(1.2)\end{array}$ & n.s. & 0.00 & $\begin{array}{c}0.6 \\
(1.2)\end{array}$ & $\begin{array}{c}0.3 \\
(0.9)\end{array}$ & $\begin{array}{c}0.4 \\
(1.0)\end{array}$ & $\begin{array}{c}0.4 \\
(1.0)\end{array}$ & n.s. & 0.01 \\
\hline Tough & $\begin{array}{c}1.1 \\
(1.4)\end{array}$ & $\begin{array}{c}1.2 \\
(1.5)\end{array}$ & $\begin{array}{c}1.3 \\
(1.6)\end{array}$ & $\begin{array}{c}1.3 \\
(1.7)\end{array}$ & n.s. & 0.00 & $\begin{array}{l}1.6^{a} \\
(1.7)\end{array}$ & $\begin{array}{l}0.5^{b} \\
(1.0)\end{array}$ & $\begin{array}{l}1.4^{a} \\
(1.6)\end{array}$ & $\begin{array}{l}1.4^{a} \\
(1.6)\end{array}$ & $* * *$ & 0.08 \\
\hline Mushy & $\begin{array}{c}0.5 \\
(1.1)\end{array}$ & $\begin{array}{c}0.9 \\
(1.5)\end{array}$ & $\begin{array}{c}0.6 \\
(1.2)\end{array}$ & $\begin{array}{c}0.7 \\
(1.3)\end{array}$ & n.s. & 0.02 & $\begin{array}{l}0.2^{b} \\
(0.6)\end{array}$ & $\begin{array}{l}1.5^{a} \\
(1.7)\end{array}$ & $\begin{array}{l}0.4^{6} \\
(1.1)\end{array}$ & $\begin{array}{l}0.5^{b} \\
(1.2)\end{array}$ & $* * *$ & 0.16 \\
\hline
\end{tabular}

Two-way ANOVA's performed on the main effects of label and recipe. Standard deviations are indicated in italics. Bold highlights indicate significant values. Significance levels indicated by ${ }^{*} p<0.05, * * p<0.01, * * * p<0.001$, and n.s. as non-significant $(p>0.05)$. Different letters denote significant differences between means of the recipes and of the labels. 

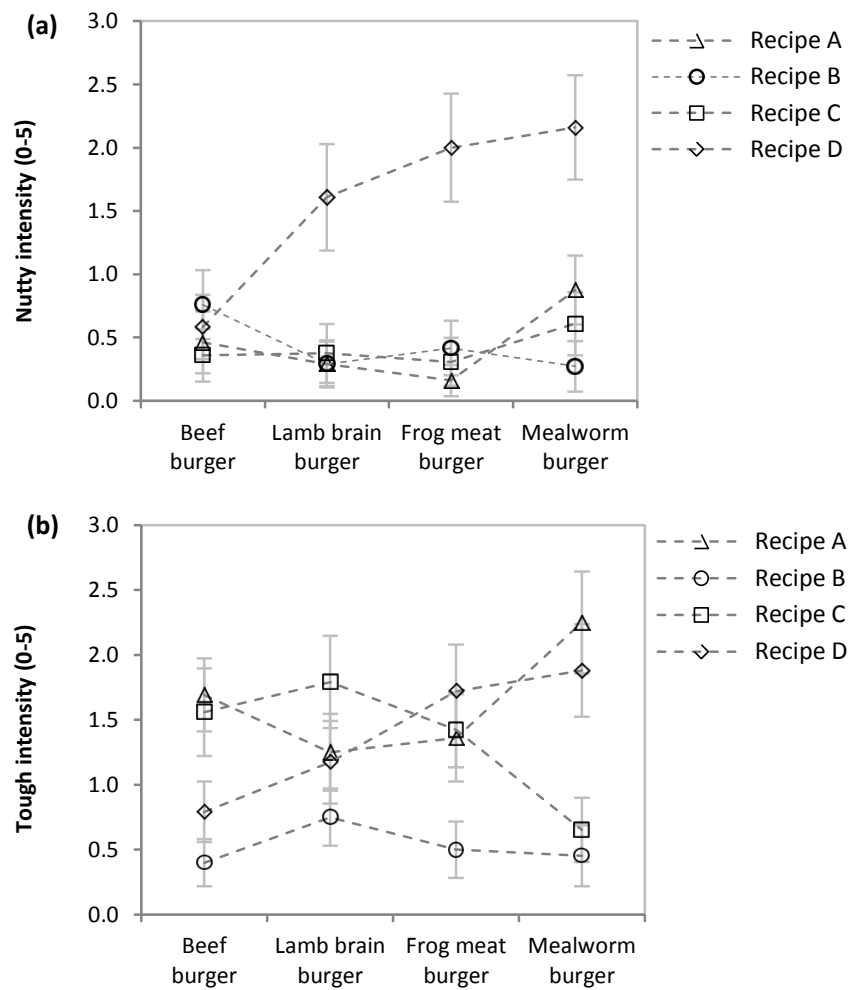

Figure 4.3. Mean attribute intensities showing interaction effects between label and recipes for nutty and tough. Error bars represent standard error. Recipe A: $94 \%$ beef, $2 \%$ breadcrumbs, $2 \%$ tofu, $2 \%$ hazelnuts; recipe B: $86 \%$ beef, $10 \%$ breadcrumbs, $2 \%$ tofu, $2 \%$ hazelnuts; recipe C: $80 \%$ beef, $2 \%$ breadcrumbs, $16 \%$ tofu, $2 \%$ hazelnuts; and recipe D: $88 \%$ beef, $2 \%$ breadcrumbs, $2 \%$ tofu, $8 \%$ hazelnuts.

\subsubsection{Correlations between sensory attributes}

Correlations between the 13 common sensory attributes that significantly differentiated the burgers both before and after tasting are visualized in the bi-plots in Figure 4.4, in order to explore how participants' use of sensory attributes changed upon tasting the product. Before tasting (Figure 4.4a), strong positive correlations were observed between attributes that were expected for beef (meaty, fatty, juicy, tender, firm), between attributes expected for lamb brain (musty, slimy, mushy) and frog meat (tough), and between attributes expected for mealworms (nutty, mealy, dry, grainy). High positive correlations were observed between some attributes that are actually opposites on a physical dimension (e.g. mushy and tough). The correlations between attributes changed after tasting (Figure 4.4b). Attributes that were related to harder and rougher textures (tough, dry, firm, grainy) were positively correlated with one another, and were negatively correlated with attributes related to softer and smoother textures (mushy, slimy, juicy, tender, fatty), where it appears that although fatty was classified as a flavour attribute, it was also used as a texture attribute. Attributes that were related to less beef-like flavour (nutty, 
musty, mealy) were positively correlated with one another, and were negatively correlated with the attribute that is more beef-like (meaty). These results provide further indication that sensory perceptions were strongly influenced by the physical properties of the sample tasted.
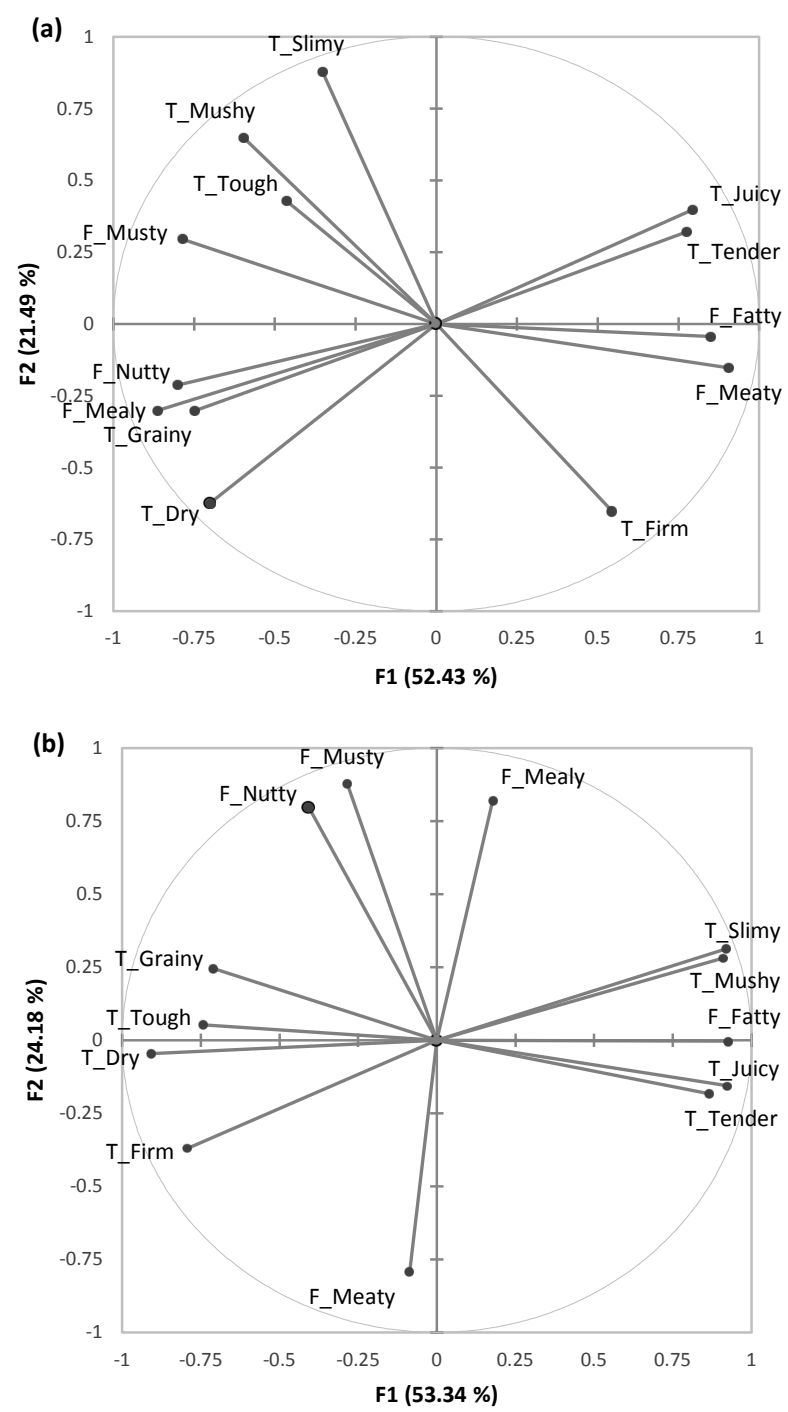

Figure 4.4. Correlation bi-plots of the 13 sensory attributes that differentiated burgers (a) before and (b) after tasting, based on mean attribute intensities of the 16 label-recipe combinations. 


\subsubsection{Food appropriateness and the willingness to eat again}

\subsubsection{Relating familiarity to food appropriateness and willingness to eat}

Familiarity levels for each ingredient and food appropriateness and willingness to eat for each familiarity level are shown in Figure 4.5.
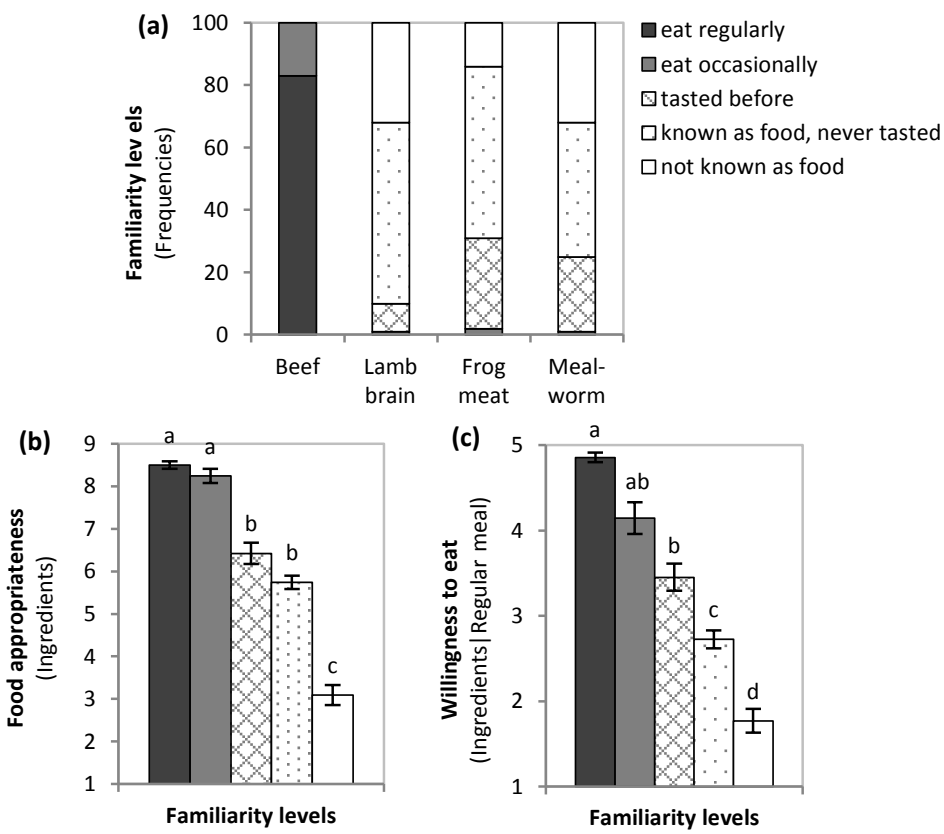

Figure 4.5. (a) Familiarity levels for novel ingredient, (b) food appropriateness and (c) willingness to eat in a regular meal per familiarity level. One way ANOVA's performed to analyse differences in food appropriateness and willingness to eat between each familiarity level. Error bars represent standard errors and different letters denote significant differences between familiarity levels.

While all participants were regular or occasional consumers of beef, hardly any of them were regular consumers of the novel ingredients, where those who have tasted them before had only limited taste experience with the ingredients (Figure 4.5a). Lamb brain and mealworms were less familiar as food than frog meat. Food appropriateness of the ingredients increased significantly with higher familiarity levels $[F(4,399)=103.60, p<.001]$ (Figure 4.5b). Recognising an ingredient's edibility related to higher food appropriateness, whereas merely tasting the food before did not increase food appropriateness if the ingredient was already known as food. Occasional and regular consumers perceived the ingredient to be higher in food appropriateness than those who have limited taste experience. Willingness to eat the ingredients in a regular meal when not incorporated within burgers also increased significantly with higher familiarity levels $[F(4,399)=84.24, p<.001]$ (Figure 4.5c). Recognising an ingredient's edibility and having tasted it 
before both significantly increased the willingness to eat the ingredient. A regular consumer is also significantly more willing to eat a food ingredient than one who has merely tasted it before.

\subsubsection{Food appropriateness and willingness to eat burgers and ingredients}

Food appropriateness differed significantly between burgers $[F(3,297)=64.02, p<.001]$, with beef burger being the most highly rated, followed by frog meat burger, and lamb brain and mealworm burgers (Figure 4.6a). This pattern follows the overall familiarity levels of the respective ingredients (see Figure 4.5a). After tasting, the 3 novel burgers were rated equally on food appropriateness, where significant increases in ratings were observed for lamb brain burger [t(99)=4.04, $p<.001]$ and mealworm burger $[t(99)=2.86, p=.005]$ but not for the frog burger $[t(99)=1.54, p=.127]$. The incorporation of ingredients within burger patties had a significant positive effect on food appropriateness of lamb brain $[\mathrm{t}(99)=6.40, \mathrm{p}<.001]$ and mealworms $[\mathrm{t}(99)=4.57, \mathrm{p}<.001]$, but not for frog meat and beef.
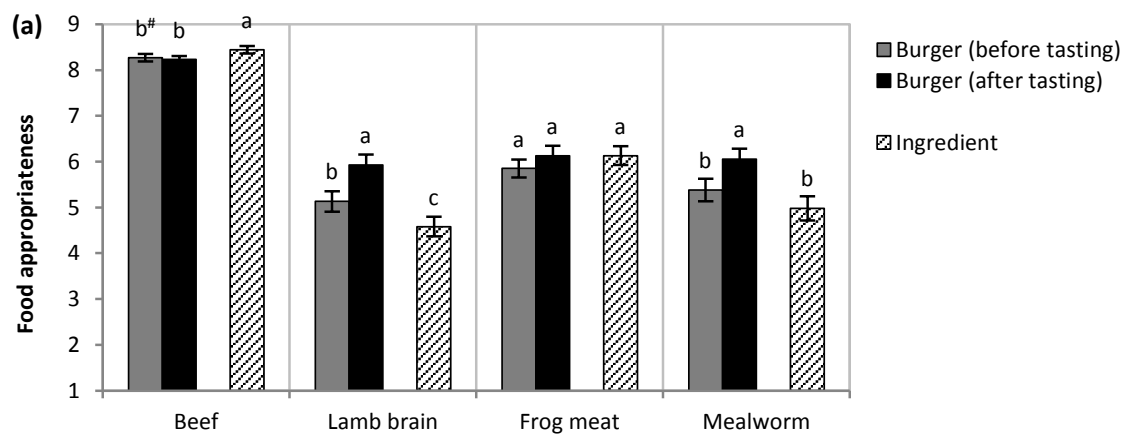

(b)
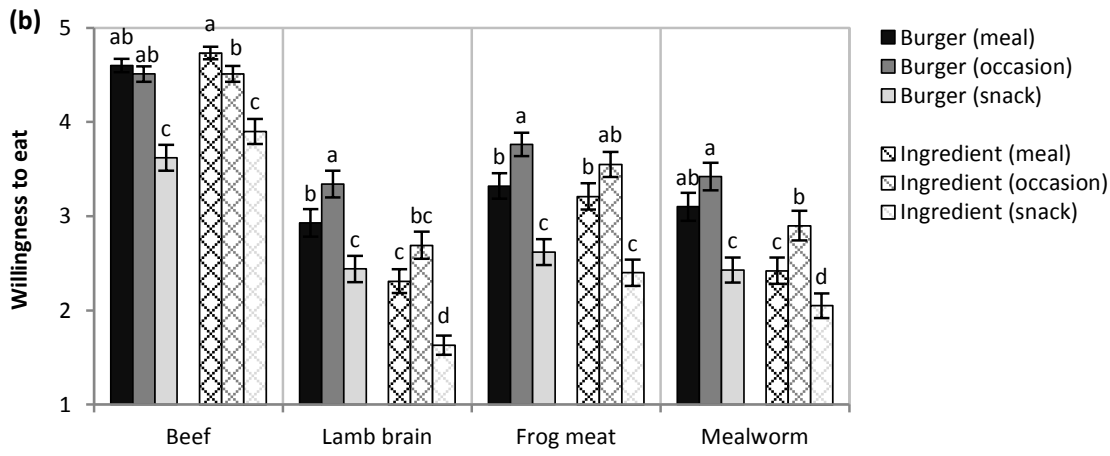

Figure 4.6. Mean (a) food appropriateness and (b) willingness to eat burgers (before and after tasting) and ingredients. Repeated measures ANOVA's performed to analyse differences between means relating to each type of food. Error bars represent standard errors and different letters denote significant differences between means. "Food appropriateness of beef burger was evaluated after tasting the beef burger but before tasting the novel burgers. 
Willingness to eat differed significantly according to the form of preparation (i.e. burger or ingredient) $[F(1,99)=25.89, p<.001]$, label $[F(3,297)=88.16, p<.001]$, and consumption situation (i.e. regular meal, special occasion, or snack) $[F(2,198)=83.05, p<.001]$ (Figure 4.6b). Incorporating novel ingredients in burgers generally increases the willingness to eat but willingness to eat novel ingredients and burgers were always rated lower than the beef and beef burger. Willingness to eat the novel ingredients and burgers was also generally higher for special occasions than for regular meal, whereas willingness to eat was lowest for the snack situation.

\subsubsection{Relating sensory perceptions to food appropriateness and willingness to eat}

Correlations of the sensory perceptions after tasting the burgers with food appropriateness and future willingness to eat the burgers again in a regular meal are shown in Table 4.3. Significant correlations were observed between 11 of 20 attributes and perceived food appropriateness, and between 9 of 20 attributes and future willingness to eat in a regular meal. Correlations were observed to be positive for positive attributes in a burger context (e.g. meaty, juicy, tender) and negative for negative attributes in a burger context (e.g. musty, bland, tough, mushy). Therefore samples that were experienced to have more of the properties related to beef, and less of the properties related to the novel ingredients received higher ratings in food appropriateness and willingness to eat after tasting.

Table 4.3. Pearson's product moment correlations between attribute intensities and food appropriateness and willingness to eat in a regular meal after samples have been tasted.

\begin{tabular}{|c|c|c|c|c|}
\hline & \multicolumn{2}{|c|}{ Food appropriateness } & \multicolumn{2}{|c|}{ Willingness to eat } \\
\hline & $r$ & $\mathrm{p}$ & $r$ & $\mathrm{p}$ \\
\hline \multicolumn{5}{|l|}{ Flavour } \\
\hline Meaty & +0.33 & $* * *$ & +0.31 & $* * *$ \\
\hline Salty & +0.09 & n.s. & +0.07 & n.s. \\
\hline Nutty & -0.09 & n.s. & -0.12 & $*$ \\
\hline Fatty & +0.00 & n.s. & -0.04 & n.s. \\
\hline Mealy & -0.10 & n.s. & -0.07 & n.s. \\
\hline Musty & -0.31 & $* * *$ & -0.19 & $* *$ \\
\hline Bitter & -0.18 & $* *$ & -0.01 & n.s. \\
\hline Roasted & +0.09 & n.s. & +0.05 & n.s. \\
\hline Bland & -0.21 & $* * *$ & -0.14 & $*$ \\
\hline Chicken & -0.08 & n.s. & -0.05 & n.s. \\
\hline \multicolumn{5}{|l|}{ Texture } \\
\hline Juicy & +0.25 & $* * *$ & +0.16 & $* *$ \\
\hline Tender & +0.40 & $* * *$ & +0.32 & $* * *$ \\
\hline Firm & +0.07 & n.s. & +0.08 & n.s. \\
\hline Dry & -0.21 & $* * *$ & -0.11 & n.s. \\
\hline Fibrous & -0.13 & $*$ & -0.18 & $* *$ \\
\hline Slimy & -0.06 & n.s. & -0.12 & $*$ \\
\hline Grainy & -0.14 & $*$ & -0.14 & $*$ \\
\hline Crunchy & +0.05 & n.s. & +0.00 & n.s. \\
\hline Tough & -0.20 & $* * *$ & -0.10 & n.s. \\
\hline Mushy & -0.17 & $* *$ & -0.10 & n.s. \\
\hline
\end{tabular}

Bold highlights indicate significant values. Significance levels indicated by ${ }^{*} p<.05,{ }^{*} p<.01, * * * p<.001$, and n.s. as non-significant ( $p>0.05)$. 
To further explore the extent to which sensory perceptions of the burgers after tasting contribute to the perceived food appropriateness and future willingness to eat in a regular meal, the 20 attribute intensities were entered into multiple regression models. Attribute intensities significantly predicted food appropriateness [model $R^{2}=0.329, p<.001$ ], and to a lesser extent willingness to eat in a regular meal [model $R^{2}=0.235, p<.001$ ]. Food appropriateness itself was a strong predictor of willingness to eat [model $R^{2}=0.398, p<.001$ ], and adding the attribute intensities did not significantly improve the model [model $R^{2}=0.456, p<.001$; change in model $\left.\mathrm{R}^{2}=0.060, \mathrm{p}=.113\right]$. These results suggest that while food appropriateness mainly predicted future willingness to eat, the sensory perceptions can still influence the perceived food appropriateness and indirectly affect future willingness to eat.

\subsection{DISCUSSION}

This study extends previous work (Chapter 3 ) by examining the sensory perceptions that underlie the hedonic evaluations using a similar experimental design. Understanding sensory perceptions of a food is important for product development, but is seldom undertaken in research on novel food acceptance. Here we explore the sensory perceptions and relate it to food appropriateness and willingness to eat to deliver new insights for product development.

Prior to tasting, sensory expectations for the novel burgers were generally negative, where attributes such as musty, mealy, slimy and mushy were used to describe the expected sensory properties. Participants seemed to infer these properties by combining their expectations of beef and novel ingredient attributes that were based more on typical associations with the items (e.g. mealworm = mealy). Evidently, beliefs regarding the sensory experience are based on the observed stimuli and existing knowledge about it (Kardes et al., 2004), which did not necessarily reflect the actual taste of the foods. Differences in expectations between those who have tasted and never tasted the unusual novel foods confirmed that prior experience greatly influences the accuracy of the inferences about the product experience (Alba \& Hutchinson, 1987; Grunert et al., 2004), where those with taste experience seemed more aware of the actual taste of the novel ingredients (e.g. mealworms are nutty instead of mealy), which also tended to be less negative. This was also observed qualitatively in Chapter 2, where Thai eaters of insects with extensive eating experiences with various insect species could tell confidently about their taste when prepared in various ways, but knew little about the taste of species they have not tasted before. While it cannot be determined from this study if these negative taste expectations affect the willingness to try, we observed that participants who rejected tasting novel burgers gave reasons relating to whether it is fit for consumption, suggesting that food appropriateness may be of greater importance. 
Upon tasting, sensory perceptions were mainly influenced by the properties of the food, which implies that the taste experience is able to easily correct misconceived ideas regarding the taste of a novel food. This finding confirms earlier research that the experienced sensory properties determine evaluations of sensory-liking when the food is consumed (Cardello \& Maller, 1982; Raudenbush \& Frank, 1999), but our results further indicate that the information of the meat composition that was provided by the label influenced the way the taste was judged. It might also influence the way the consumer selects applicable words to describe the experience (e.g. higher ratings of mealy when samples were presented as a mealworm burger). In addition, an interesting interaction effect was observed in the evaluations of nutty flavour. The recipe that contained extra hazelnuts and that was perceivably nuttier in flavour, was only evaluated to be more nutty when presented with a novel burger label, but not when presented with a beef burger label. As only the mealworm burger and not the other novel burgers were expected to be high in this attribute, this might not be a case of assimilation with expectations (PiquerasFiszman \& Spence, 2015; Schifferstein, 2001), but due to consumers holding on less strongly to their pre-conceived beliefs about the product experience when product knowledge is lacking (Spreng \& Page, 2001).

Outcomes from the sensory perceptions provide little evidence for why novel foods are less tasty than familiar foods after tasting (Raudenbush \& Frank, 1999) as consumers appear to easily learn the taste of a food. However, consistent with previous findings (Chapter 3), we found that food appropriateness and not the experienced taste determined future willingness to eat these novel foods. This may be because of the taste similarity between the samples, where other factors tend to play a larger role in determining the willingness to eat a food again when products do not differ much in terms of taste (Grunert \& van Trijp, 2014). This might also explain why consumer liking and willingness to pay for products tend to be influenced more by the information on the labels (e.g. organic production, brands) than its actual taste (Lange, Martin, Chabanet, Combris, \& Issanchou, 2002; Napolitano et al., 2010; Napolitano et al., 2013). These observations do not necessarily indicate that taste is irrelevant and unimportant, as the experienced sensory properties still contributed significantly to food appropriateness, thereby indirectly influencing the willingness to eat.

The common approach recommended for improving the sensory appeal of novel foods is by disguising it within familiar products (Gmuer et al., 2016; Tuorila, Andersson, et al., 1998; Wansink, 2002). This study showed that by doing so it creates more positive taste expectations, where negative attributes related to the novel foods become less pronounced. Food appropriateness and willingness to eat were also higher when the novel foods are incorporated in burger patties. Hence, this approach may work in terms of lowering the initial barriers to trying a novel food, but its true effectiveness also depends on other factors. Higher perceived intensities of attributes related to beef (e.g. meaty, tender) and lower perceived intensities of attributes related to the 
novel are likely to have been categorized and judged according to quality standards of the product category (van Trijp \& van Kleef, 2008), meaning that adding novel foods to familiar products may lead consumers to expect the novel product to taste similar to the original and increase the likelihood of being disappointed with its taste (Chapter 6). In addition, knowledge of the presence of culturally inappropriate foods within a product is already sufficient to result in its rejection, even if the novel food's properties are not perceivable (Rozin \& Fallon, 1987), as several participants in this study declined to taste novel burgers even though it did not look any different from a regular burger patty.

\subsubsection{Implications on product development}

Choosing a product preparation that is more likely to override negative expectations could positively influence the willingness to try and learn the properties of a novel food. For instance, a mealworm that is expected to have a slimy and mushy texture is likely to have higher sensory appeal when fried rather than boiled in a soup, since prior knowledge pertaining to these methods of preparation would indicate that soft foods turn crispy when fried, but could be mushy when water is taken in during boiling. Even though the physical properties of the product strongly determined sensory perceptions during tasting, the experience of these properties is subject to an individual's interpretation. In this study, expectations due to the labelled content of the sample influenced the way the experience was evaluated. Attributes related to the novel burgers (e.g. mealy, mushy), although not typically used to describe beef burger patties, were still considered applicable when describing the taste of the samples labelled to be novel burgers. As a result, ratings of attributes such as slimy and mushy were higher when texture was softer and lower when texture was harder, whereas ratings of mealy and musty were higher when flavour was less meaty and lower when flavour was more meaty. Hence, the sensory appeal of novel foods could benefit from developing products that not only perform well according to quality standards of the product category (van Trijp \& van Kleef, 2008), but that also disconfirms negative sensory expectations evoked by the unusual novel foods.

In addition, the positioning of the novel food could pose restrictions and create dilemmas when developing products from culturally novel foods. For instance, the current promotion of insects as a meat alternative has created expectations regarding the use of insects in similar ways to meat (Chapter 2 \& Chapter 6). On one hand, using insects as a meat substitute would create expectations that insects should taste like meat, which might not be possible to achieve given the insect's properties (Chapter 6; Deroy et al., 2015; Hoek et al., 2011). On the other hand, the more nut-like than meat-like flavour and texture of insects like mealworms may be more positively experienced when prepared in products that fit better with nuts than with meat. Yet the use of insects within cakes and cookies that go well with nuts may not match the environmental motivations for eating insects (Chapter 2; Shelomi, 2015). Developing products from novel foods 
would thus require more extensive work than simply hiding the novel foods within familiar products, where the choice of product and adjustment of its properties should also fit with consumer motivations and taste expectations of the product category.

\subsubsection{Limitations \& future research}

In this study, the unusual novel foods were not actually present in the products tasted. Hence, it is not possible to draw conclusions here regarding the actual sensory qualities of these novel foods. We chose for this deceptive study design as we wanted to recruit regular consumers and not adventurous eaters for this study, and still be able to randomize the presentation of labels with recipes without causing participants to ingest foods that they would not be comfortable with eating. While two other sensory studies have managed to conclude on the inferior sensory quality of insect-based burgers (Caparros Megido et al., 2016; Schouteten et al., 2016), they do not offer explanations on how the sensory quality can be improved. Our study design provides actionable insights when developing products from unusual novel foods like insects, by exploring the basis of the sensory perceptions and how it contributes to acceptance.

Furthermore, sensory-liking was not investigated in this study, which could have otherwise allowed its relations with sensory perceptions to be studied. CATA-type consumer descriptive analysis could be combined with hedonic ratings to provide more useful information regarding consumer preferences for specific product attributes (Jaeger \& Ares, 2014). In order to develop attractive products from novel foods, more research needs to be done to relate consumer perceptions to the physical properties of the product, where studies with the real ingredients are required and consumer populations representative for the general population could be used. Lastly, products used in this study were generally good tasting, which is likely to have limited the influence of sensory aspects on willingness to eat. If the sensory quality of the burger patties had ranged from extremely good to extremely bad, it would have provided better insights into the impact of sensory perceptions on the potential acceptance of such foods.

\subsection{CONCLUSIONS}

This study examined the sensory perceptions that underlie the lack of sensory appeal of culturally unusual foods like insects and discussed the implications for product development. Beliefs about the food's taste were informed by species-related associations, and tended to be more negative when the food had never been tasted before. While these pre-consumption beliefs had some influence on sensory perceptions after tasting, the food's properties mainly determined the consumption experience. However, the low willingness to eat had more to do with food appropriateness than the actual taste of the food. The experienced taste nevertheless exerts an influence on the perceived food appropriateness and should not be neglected. Outcomes 
showed that incorporating unusual novel foods within a familiar product could help to create more positive expectations, its actual taste and food appropriateness may be less attractive than the original products that consumers are familiar with. We conclude that future research on novel food acceptance should take into account that perceptions differ between novel and familiar foods, and that product development for culturally inappropriate foods would require selecting suitable product types and adjustment of its properties to match both consumer motivations and taste expectations.

\section{ACKNOWLEDGMENTS}

Our appreciation goes to Floor Kroese and Zeena Harakeh of Utrecht University for providing the use of the living room laboratory that was specially designed for experimental and observational research. We also thank the students of Wageningen University for helping with the logistics of the study. Many thanks go to Betina Piqueras-Fiszman and the anonymous reviewers for their comments that helped to improve the manuscript. 


\section{CHAPTER 5 Proouct's ROLE}

The influence of product preparation, familiarity and individual traits on the consumer acceptance of insects as food

Hui Shan Grace Tan

Eva van den Berg

Markus Stieger 


\section{ABSTRACT}

Insects are highly valued as food in many cultures but have only recently gained interest in the West as a sustainable alternative to reduce the environmental impact of meat production. Despite the growing consumer interest in insect consumption, there is still a great disparity between curious trying and actual acceptance. The aim of this study was to examine how the product preparation, familiarity and individual traits (e.g. food neophobia) influence the consumer acceptance of insects as food.

Dutch consumers $(n=976)$ evaluated 8 mealworm product images on 4 acceptability measures (product appropriateness, expected sensory-liking, willingness to buy, willingness to try). Product images varied according to mealworm visibility (visible/invisible), carrier flavour (savoury/sweet) and carrier origin (Western/Asian).

High product acceptability was not simply achieved by adding mealworms to familiar foods. Acceptability depended very much on the perceived appropriateness of mealworms as food and the perceived appropriateness of the product combination. However, mealworm products were always expected to be inferior to the carrier products, even when visually identical. Familiarity with mealworms and individual traits played a relatively minor role, and influenced the willingness to try more than the other acceptability measures.

We conclude that appropriate product design is important but insufficient to achieve consumer acceptance of insects as food in the West. Additional incentives are required to encourage acceptance beyond the mere willingness to try. We discuss the complexities underlying the consumer acceptance of insects as food and reflect on how acceptance might be increased in the future. 


\subsection{INTRODUCTION}

Rapid population growth and rising meat consumption have spurred efforts to improve current food production systems and to develop more sustainable food alternatives (Boland et al., 2012). For this reason, edible insects have gained much attention for their high nutritional value and environmental advantages over meat production (van Huis, 2013). Their short life cycles, low space requirements, efficient nutrient conversion rates and lower greenhouse gas production render insects to be, in principle, an excellent alternative to meat (van Huis, 2013). Yet even though a large variety of insects are valued as tasty delicacies in many cultures around the world (Bukkens, 1997; Hanboonsong, 2010; Luo, 1997), the vast majority of Western consumers reacts with disgust at the prospect of ingesting creatures that are more familiar as pests than as food (Harris, 1985; Looy et al., 2014). At the moment, many cultural and psychological barriers stand in the way of consumer acceptance of insects as food in Western cultures (Lensvelt \& Steenbekkers, 2014; Looy et al., 2014).

Efforts to overcome the disgust towards insect consumption have so far focussed on reasoning away the prejudices. Yet marketing foods on a rational basis usually has limited success. Ethical arguments have proven to be ineffective in persuading consumers to fully accept more sustainable meat alternatives (de Boer et al., 2013). Consumers are generally unwilling to sacrifice the immediate pleasures of the food experience for uncertain and delayed benefits that are not necessarily accrued to the self (Griskevicius, Cantú, \& Vugt, 2012; van Trijp \& Fischer, 2011). While these arguments have contributed to an increase in interest in insects as food, few Western consumers are actually ready to adopt insects as a food as part of their regular diet (Chapter 2; Looy et al., 2014; Tucker, 2014). The lack of acceptance has often been attributed to the low sensory appeal and unfamiliarity with insects as food (Chapter 2; Deroy et al., 2015; Shelomi, 2015; Tucker, 2014). This highlights the importance of first overcoming existing negative perceptions regarding insect-based foods if consumer acceptance in Western cultures is to be achieved (Looy et al., 2014; Shelomi, 2015). Creating appealing products could thus play a critical role in the acceptance of insects as food (Chapter 2; Deroy et al., 2015).

The starting point for consumer-oriented product development is an understanding of consumer expectations and preferences (van Trijp \& Steenkamp, 2005). However, the unfamiliarity with insects as food in Western cultures may pose difficulties for product development, as prior taste experiences are required in order for preferences to be formed (Birch, 1999). Consumers from insect-eating cultures have distinct expectations regarding how insects are appropriately prepared (Chapter 2; Chakravorty et al., 2011; Obopile \& Seeletso, 2013). Such expectations are less distinct in cultures where insect consumption is not common (Chapter 2). Several studies have shown that the product preparation affects the willingness to eat insects (Chapter 2; Caparros Megido et al., 2014; Hartmann et al., 2015). But to date, consumer expectations and 
preferences towards different aspects of the product preparation have not been investigated. This study aims to provide actionable insights for the development of insect-based foods by examining how product preparation influences different dimensions of acceptability.

Using familiar preparations has been shown to effectively increase the liking and willingness to eat novel foods (Pelchat \& Pliner, 1995; Tuorila, Andersson, et al., 1998; Wansink, 2002). Exceptions arise when the combination of ingredients is perceived to be inappropriate (Stallberg-White \& Pliner, 1999). This means that ingredients could be liked separately but disliked in combination (Cardello et al., 2000; Schutz \& Martens, 2001). For instance, a qualitative study involving Dutch consumers (Chapter 2) showed that grasshoppers were perceived to be appropriately flavoured with chilli and salt, but inappropriately flavoured with chocolate due to perceived mismatch of sensory properties or mismatch with expectations of insects to be used as meat substitutes. This subsequently impacted the willingness to try and buy the insect-based foods. Apart from the type of preparation, presenting insects invisibly within familiar foods could improve acceptance (Chapter 2), as it has the advantage that the products would not look different from regular foods (Shelomi, 2015). However, in the case of insects, the mere idea of its presence within the food could be perceived as a contamination of the original food and lead to rejection (Rozin \& Fallon, 1987). Apart from a previous study that compared insect-based burgers to standard beef burgers (Chapter 3), no other studies have compared the acceptability of insect-based foods to the corresponding insect-free foods. Hence, this study not only examines the influence of appropriateness of the product preparations on acceptability, but also compares the evaluations of insect products to the original products in order to assess their potential alongside existing products on the market.

An individual's familiarity with a food and level of food neophobia are known to be key determinants of acceptance or rejection of various novel foods (Fenko, Backhaus, \& van Hoof, 2015; Raudenbush \& Frank, 1999; Tuorila et al., 2001) including insects (Hartmann et al., 2015; Verbeke, 2015). Absence of taste experience and higher levels of food neophobia have been consistently linked to lower hedonic ratings and consumption intentions (Raudenbush \& Frank, 1999; Tuorila et al., 2001). This may be attributed to the uncertainty and fear of unknown foods (Rozin \& Vollmecke, 1986; Tuorila et al., 1994), as well as misconceived notions about the expected sensory experience when taste experience is absent (Alba \& Hutchinson, 1987). In the case of insects, strong negative associations with the origins and nature of the insects tend to be brought up when Western consumers are confronted with insect-based foods (Chapter 2). In addition, socio-demographic characteristics could play a role in consumer acceptance, where younger males have been shown to be more open to unusual foods like insects (de Boer et al., 2013; Hartmann et al., 2015; Verbeke, 2015). Yet consumers who are keen on tasting and have previously tasted insects still express difficulties in accepting insects as food (Chapter 2; Tucker, 2014). Little is known about the importance of these individual-related factors in determining 
the consumer acceptance of insect-based foods. This study thus examines the influence of taste familiarity, food neophobia, and socio-demographic characteristics on various acceptability measures in relation to the product-related factors.

The mixed attitude of interest and disgust that Western consumers exhibit towards insect consumption further adds a layer of complexity to the study of consumer acceptance of insects as food. Many consumers are willing to try despite their negativity, but are unwilling to adopt insects as food even if they recognized their functional benefits and good taste (Chapter 2; Tucker, 2014). Clearly, there is a distance between curious trying and actual acceptance of insects as a regular part of the diet. While this has been highlighted in previous studies (Chapter 2 \& Chapter 3), the difference between curious trying and product acceptance has not yet been measured. This study thus explores the consumer acceptance of insect-based foods using four different measures of acceptability-(1) perceived appropriateness of the product combination, (2) expected sensory-liking, (3) willingness to buy, and (4) willingness to try. We hypothesize that the perceived appropriateness of the product combination (i.e. insect + carrier product) influences the expected-sensory liking of the insect-based product (Cardello et al., 2000), and these sensory-affective expectations are likely to subsequently affect intentions to consume a food (Mela, 2001b). Willingness to buy is often measured when investigating the introduction of novel food products (Solheim \& Lawless, 1996) as an indication of how much a consumer values a product (Beneke, Flynn, Greig, \& Mukaiwa, 2013). We therefore included it to provide a view of consumer acceptance that goes beyond the mere willingness to try.

This study aims to provide new insights into consumer acceptance of insects as food by examining the role of various product- and individual-related factors on various measures of acceptability. Choosing for a species that is more familiar in the local context and incorporating it in familiar products might be a good way to introduce insects as food in Western cultures (Chapter 3; Shelomi, 2015). Mealworms (Tenobrio molitor larvae), which are available for human consumption in Dutch supermarkets, were incorporated in whole and ground forms within carrier products that are available in Dutch supermarkets, and product images were evaluated.

\subsection{MATERIALS AND METHODS}

\subsubsection{Participants}

Participants were recruited from a Wageningen University research database (6161 people). A total of 1173 subjects responded to an email asking for Dutch consumers of at least 18 years of age to participate in a consumer study regarding new food products. The survey was completed by 1057 participants, of which 81 vegetarians were excluded from the analysis as some vegetarians may categorically reject meat-based carriers and mealworms based on their meal practices. 
The remaining 976 participants ranged from 18 to 94 years ( $M=44.8$ years, SD=23.1 years), of which $66.4 \%$ were female, $17.9 \%$ had secondary and $82.1 \%$ tertiary education, and $38.8 \%$ were students or employees of Wageningen University.

\subsubsection{Product images}

Table 5.1. Product images used in the study.

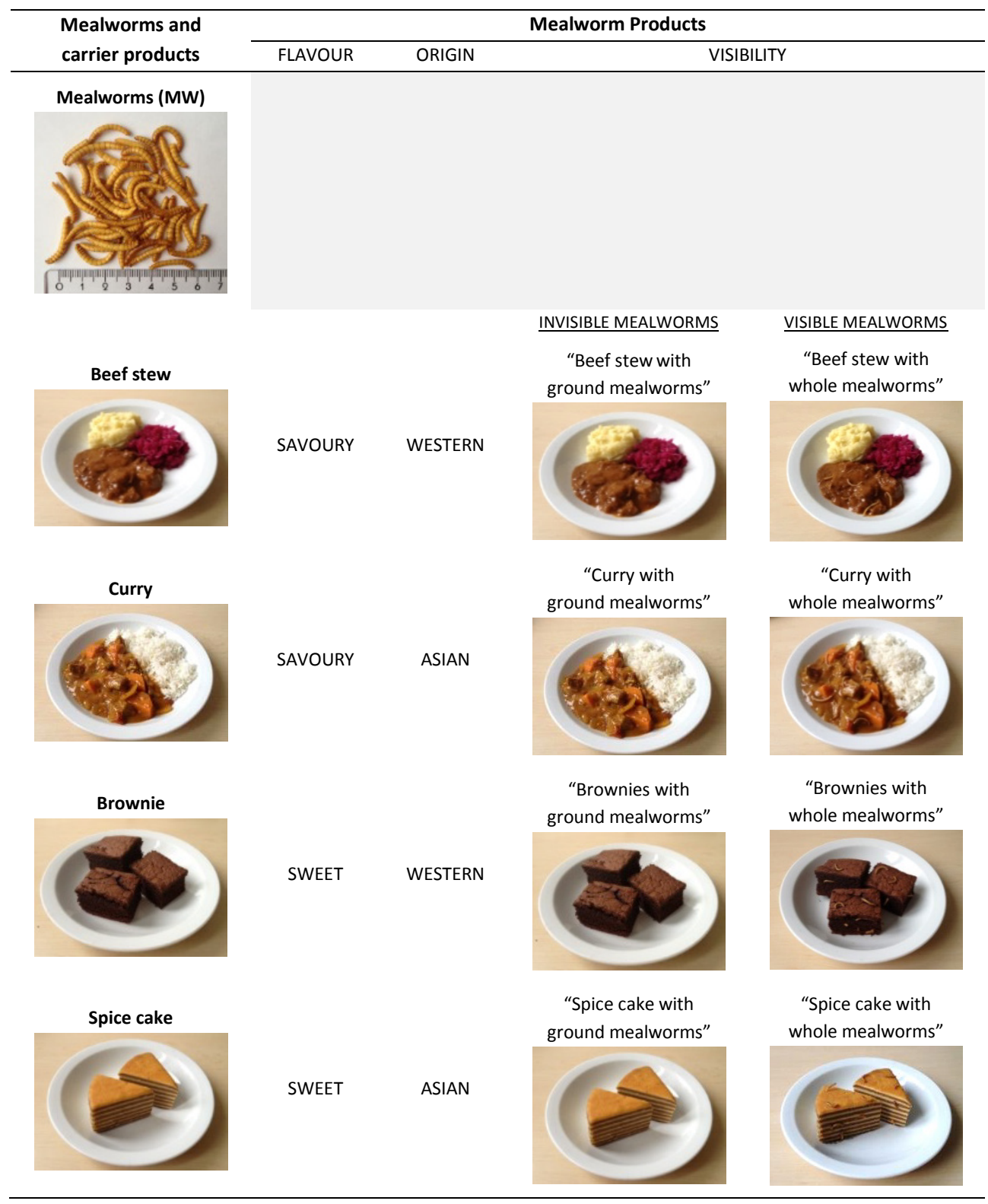


Mealworm products were systematically varied on 3 factors at 2 levels each $(2 \times 2 \times 2)$ : mealworm visibility (VIS: visible/invisible), flavour of carrier product (FLAV: savoury/sweet), and origin of carrier product (ORIG: Western/Asian) (Table 5.1). A preliminary survey with Dutch participants $(n=81)$ revealed that a clear majority preferred invisible mealworms, and for the use of savoury carriers of Asian origin, hence varying these product factors was expected to create differences in the acceptability of mealworm products. Selection of the carrier products was based on a second preliminary survey $(n=60)$ that aimed at identifying carrier products that best represent the factor levels. Products were selected when at least two-thirds of the participants had correctly classified them according to flavour and origin. The four carriers were Dutch beef stew (savoury, Western), curry (savoury, Asian), brownie (sweet, Western), and Indonesian spice cake (sweet, Asian), all of which are available in Dutch supermarkets. In the Netherlands, the beef stew is known as 'hachee' and the Indonesian spice cake is known as 'spekkoek', and these specific names were used in the survey.

All products evaluated were presented with labelled colour images (Table 5.1). Dried mealworms were purchased from a Dutch supermarket and photographed together with a ruler to indicate its size. Carriers were purchased ready-made from the supermarket, and photographed on a white plate. The same images were used for the carriers as well as the carriers with visible mealworms, mealworms were mixed or inserted into the brown component of the carrier and photographed on a white plate. Images were all presented with a description of its content, e.g. 'brownie' (carrier), 'brownies with ground mealworms' (invisible mealworms), 'brownies with whole mealworms' (visible mealworms).

\subsubsection{Survey procedure}

The survey was designed in English and translated into Dutch by a native speaker. It consisted of 3 parts (Figure 5.1): (1) evaluations of mealworms and carriers, (2) evaluations of the mealworm products, and (3) the measurement of individual traits.

In part 1, images of the four carrier products were presented with their descriptions at the top of the page. Sensory-liking of carrier products (Carrier-LIKE) was evaluated with the question "How tasty do you think this product will be?", and carrier familiarity was evaluated with the question "How familiar is this product to you?" Carrier-LIKE was evaluated on a 9-point Likert scale, anchored from 'extremely not' to 'extremely', and carrier familiarity was evaluated on a 5-point category scale, with labels according to (Tuorila et al., 2001)(1=not known, 2=known but never tasted, 3 =tasted before, $4=$ =at occasionally, and $5=$ eat regularly). Participants were then presented with an image of mealworms and an introductory text:

"Mealworms are insects that can be sustainably produced and are rich in proteins. Insects are regularly consumed by people in many countries, but their consumption is not yet so known in the Netherlands. Recently, insect-based products are being sold in Dutch supermarkets." 


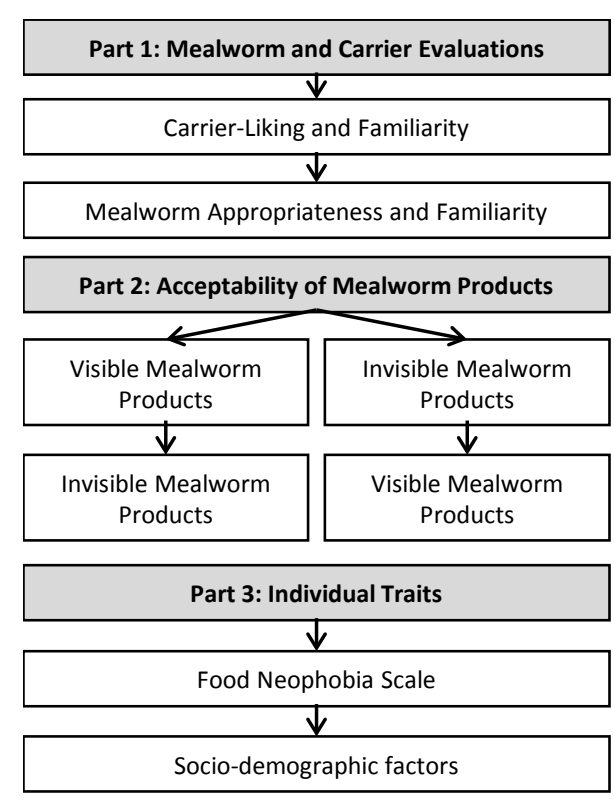

Figure 5.1. Survey procedure.

Participants evaluated the perceived appropriateness of mealworms as food (MW-APP) with the question "How appropriate do you think mealworms are for consumption?" as well as their familiarity with mealworms as food. MW-APP was evaluated on a similar 9-point as Carrier-LIKE, and mealworm familiarity was evaluated on an equivalent scale as carrier familiarity.

In part 2, eight mealworm products were evaluated with 4 acceptability measures: product appropriateness (APP) "How appropriate do you think is the following product combination?", expected sensory-liking (LIKE) "How tasty do you think is the following product combination?", willingness to buy (BUY) "Imagine that you are doing your grocery shopping and the price of the products with mealworms are the same as that of the products without. How willing are you to buy it?", and willingness to try (TRY) "Imagine that there is a free tasting session, how willing are you to taste such a product combination?" Acceptability measures were rated on a 9-point Likert scale, anchored from 'extremely not' to 'extremely'. Half of the participants first evaluated the invisible mealworm products followed by the visible mealworm products, whereas the other half received the reverse order.

In part 3, participants filled in a Dutch-translated version of the Food Neophobia Scale (FNS) (Pliner \& Hobden, 1992) and reported their age, gender, highest education level, affiliation with Wageningen University, if they were vegetarian and their reasons for being vegetarian. They also reported their preferences regarding the preparation of mealworms (visible vs invisible; savoury vs sweet; Asian vs Western) at the end of the survey. Twenty cash vouchers worth 20 euros were raffled among respondents who completed the survey. 


\subsubsection{Data analysis}

Responses on Carrier-LIKE, MW-APP and mealworm product acceptability measures (APP, LIKE, BUY, TRY) were transformed into numbers (1-9). The first two categories of familiarity where prior taste experience is absent (not known, known but never tasted) were grouped together as 'never tasted'. The next three categories of familiarity where there is prior taste experience (tasted before, eat occasionally, eat regularly) were grouped together as 'tasted before' to create a binary measure for taste familiarity of carriers (TasCarrier) and mealworms (TasMW). Ratings of FNS were reversed on items 1, 4, 6, 9, 10, and the sum of scores tabulated according to Pliner and Hobden (1992). Scores were further split into low-, mid-, and high-FNS groups using cutoff points of 1 standard deviation above and below the mean (Tuorila et al., 2001) to allow an examination of the differences between neophobic and neophilic individuals and to test and visualize the interactions between FNS and TasMW.

Pearson's product moment correlations were used to analyse the relations between the acceptability measures. Hierarchical linear regressions were performed to assess the effects of the product preparation and individual experiences and traits in predicting the four acceptability measures. Outliers (standardized residuals $\geq 3.29$ ) were removed and the remaining data reasonably met assumptions of no multicollinearity, homoscedasticity, linearity, and independence and normality of residuals. Differences in Carrier-LIKE and MW-APP between familiarity levels were analysed using independent samples t-tests. Effects of the presence of mealworms on expected sensory-liking of products was analysed using repeated measures two-way analysis of variance (ANOVA) and the change in sensory-liking (LIKE - Carrier-LIKE) was computed and its relations with Carrier-LIKE, MW-APP and APP were analysed with Pearson's product moment correlations. Effects of mealworm visibility and carrier type on acceptability measures were analysed using repeated measures three-way ANOVA ( 2 VIS $\times 2$ FLAV $\times 2$ ORIG). The effects of food neophobia levels and mealworm taste familiarity (TasMW) were analysed using two-way ANOVA (3 FNS $\times 2$ TasMW), with age, gender and education level as covariates. Bonferroni corrections were applied to all post-hoc tests.

\subsection{RESULTS}

The measures of acceptability of the mealworm products (APP, LIKE, BUY, TRY) were all highly correlated with one another [Pearson's $r=+.64$ to $+.79, p<0.001$ ], but were differentially influenced by the factors related to the product preparation (Carrier-LIKE, MW-APP, VIS, FLAV, ORIG), taste familiarity with carriers and mealworms (TasCarrier, TasMW), and individual traits (FNS, socio-demographic). Hierarchical linear regression analysis was conducted to examine how these factors influence the 4 measures of acceptability of the mealworm products (Table 5.2). 


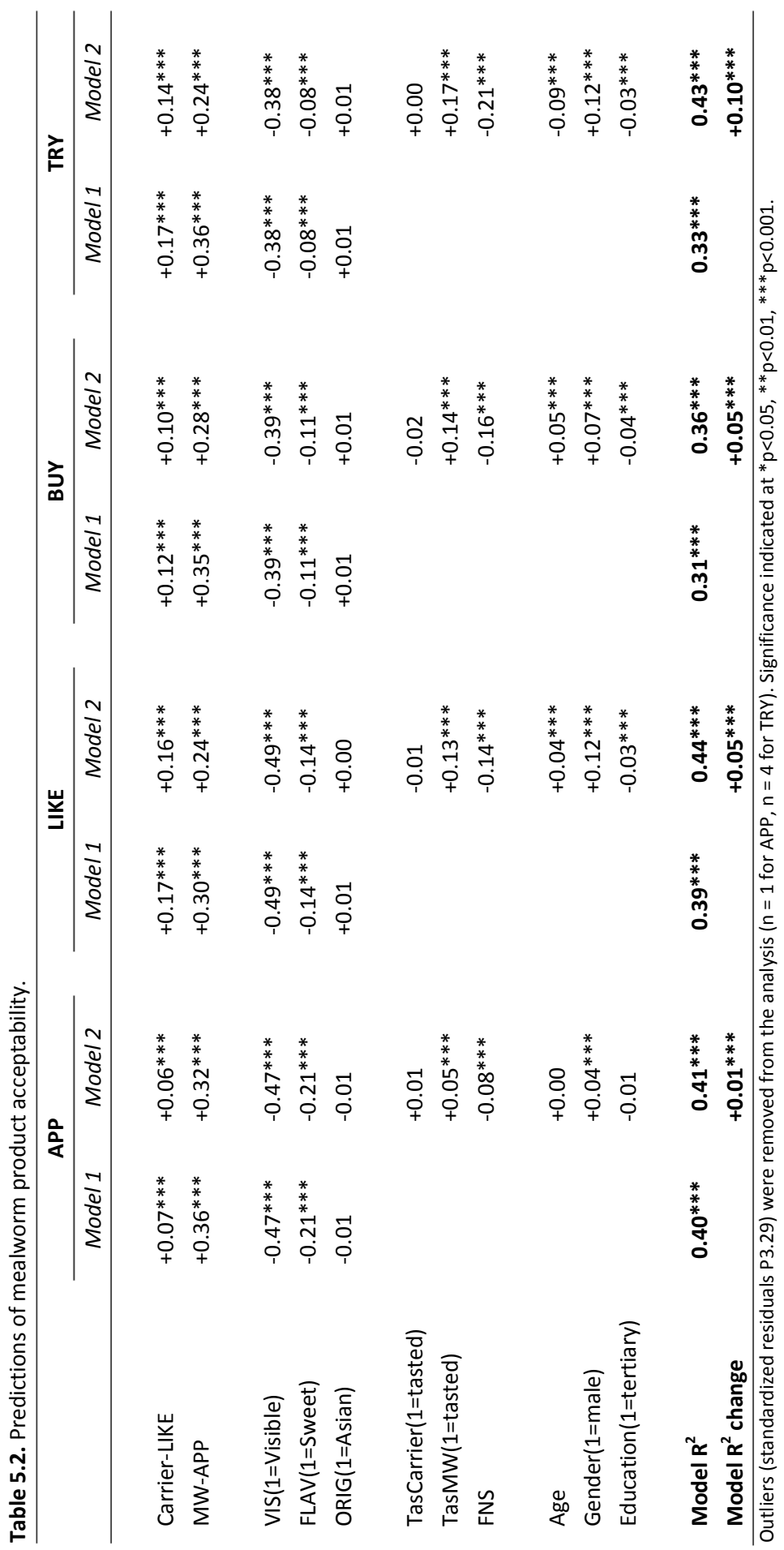


Factors related to the product preparation generally played a more major role in determining acceptability than taste familiarity and individual traits, but to varying extents across acceptability measures. From the model $\mathrm{R}^{2}$ values, product preparation factors accounted for approximately 40 times more variance the factors of familiarity and individual traits for the measure of APP. For the other measures, it was approximately 8 times more for LIKE, 6 times more for BUY, and 3 times more for TRY. Factors related to the product preparation had a greater impact on APP and LIKE than on the behavioural intentions of BUY and TRY [model 1: $R^{2}=0.40$ (APP), 0.39 (LIKE), 0.31 (BUY), 0.33 (TRY)]. Factors related to taste familiarity and individual traits had a greater impact on TRY than on the other acceptability measures [ $\triangle R^{2}=0.10$ (TRY), 0.05 (BUY, LIKE), 0.01 (APP)]. The factors examined in this study accounted for 36 to $44 \%$ of the total variance in the data. Effects of the influencing factors will be explored in greater detail in the following sections.

\subsubsection{The influence of the product preparation on acceptability}

Sensory-liking of the carrier products (Carrier-LIKE) significantly influenced the acceptability of mealworm products across all measures (Table 5.2). Higher Carrier-LIKE resulted in more positive ratings for all acceptability measures. However, its influence on acceptability was weaker than the perceived appropriateness of mealworms as food (MW-APP). MW-APP was a 2 to 5 times stronger predictor of acceptability than Carrier-LIKE. However, when sensory-liking of mealworm products (LIKE) was compared to Carrier-LIKE, mealworm products were always expected to be lower in sensory-liking, even when they were visually identical (Figure 5.2).

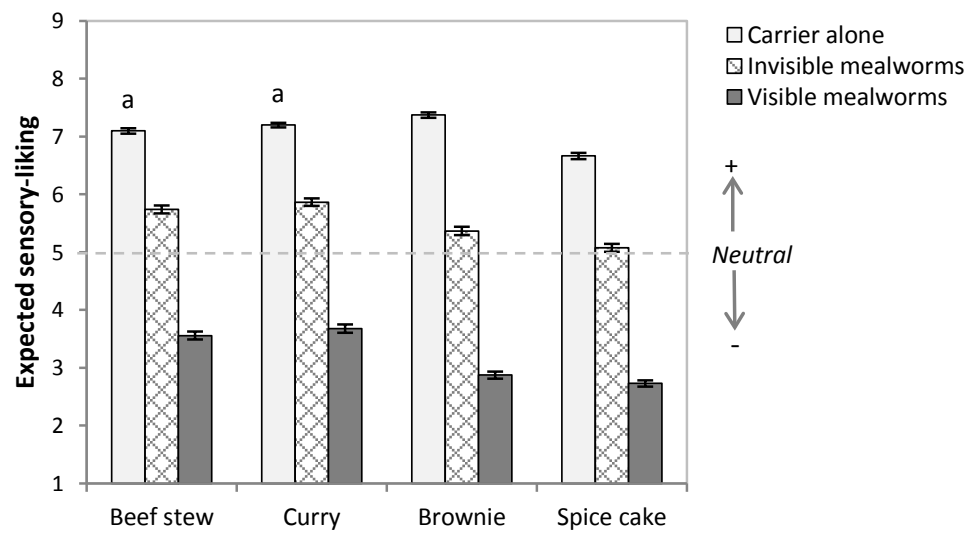

Figure 5.2. Expected sensory-liking of carrier products and mealworm products. Error bars indicate standard errors. Means were all significantly different from one another $(p<0.05$, Bonferroni) except where the same letters are indicated.

The change in expected sensory-liking when mealworms were added (LIKE - Carrier-LIKE) was significantly correlated with MW-APP, Carrier-LIKE and the perceived appropriateness of the 
product combination (APP). The change in expected sensory-liking was more negative (i.e. greater drop in expected sensory-liking) when MW-APP was lower [Pearson's $r=+.23, p<.001$ ], when Carrier-LIKE was higher [Pearson's $r=-.39, p<.001$ ], and especially when APP was lower [Pearson's $r=+.63, p<.001]$. These outcomes suggest that incorporating mealworms within a highly liked carrier product may have a limited positive effect on acceptability if the perceived appropriateness of mealworms as food and the perceived appropriateness of the product combination are low.

The effects of the three product attributes (VIS, FLAV, and ORIG) on the four acceptability measures are shown in Table 5.3. Mealworm visibility (VIS) played an especially important role in the acceptability of mealworm products. Incorporating mealworms invisibly had a strong positive effect on acceptability $\left[\eta_{p}{ }^{2}=.51\right.$ to .61$]$, where invisible mealworm products were rated more positively than visible mealworm products across all measures of acceptability [APP:+2.4, LIKE:+2.3, BUY:+1.9, TRY:+2.1] (Figure 5.3). Moreover, as shown in Figure 5.2, in comparison to the carrier products, visible mealworm products were always rated significantly lower in expected sensory-liking by an average of 3.9 points $\left[F(1,975)=3864.7, p<.001, \eta_{p}{ }^{2}=.80\right]$, and invisible mealworm products were always rated significantly lower in expected sensory-liking by an average of 1.6 points $\left[F(1,975)=742.1, p<.001, \eta_{p}{ }^{2}=.43\right]$. Participants were generally more positive when rating TRY than BUY. As a result, preparing mealworms in an invisible manner improved TRY from negative to positive, but improved BUY only from negative to neutral. This suggests that reducing insect visibility might be sufficient to lower the barriers of trying but is likely to be insufficient to persuade consumers to buy it.

Table 5.3. Effects of product preparation factors on acceptability of mealworm products.

\begin{tabular}{|c|c|c|c|c|c|c|c|c|}
\hline & \multicolumn{2}{|c|}{ APP } & \multicolumn{2}{|c|}{ LIKE } & \multicolumn{2}{|c|}{ BUY } & \multicolumn{2}{|c|}{ TRY } \\
\hline & $\mathrm{p}$ & $\eta_{p}^{2}$ & $p$ & $\eta_{p}^{2}$ & $p$ & $\eta_{p}^{2}$ & $\mathrm{p}$ & $\eta_{p}{ }^{2}$ \\
\hline \multicolumn{9}{|l|}{ Main effects } \\
\hline VIS & $* * *$ & .60 & $* * *$ & .61 & $* * *$ & .51 & $* * *$ & .53 \\
\hline FLAV & $* * *$ & .34 & $* * *$ & .24 & $* * *$ & .17 & $* * *$ & .13 \\
\hline ORIG & $* * *$ & .04 & $* *$ & .01 & n.s. & .00 & $*$ & .01 \\
\hline \multicolumn{9}{|c|}{ 2-way interactions } \\
\hline VIS*FLAV & $* * *$ & .05 & $* * *$ & .02 & $* * *$ & .01 & $* * *$ & .05 \\
\hline VIS*ORIG & $* * *$ & .04 & $* *$ & .01 & $*$ & .01 & $*$ & .01 \\
\hline FLAV*ORIG & $* * *$ & .11 & $* * *$ & .07 & $* * *$ & .03 & $* * *$ & .04 \\
\hline
\end{tabular}

Repeated measures three-way ANOVA's performed on the main and two-way interaction effects of visibility, flavour and origin. Significance indicated at $* p<0.05$ level, $* * p<0.01, * * * p<0.001$, and n.s. indicates non-significant ( $p>0.05)$.

Carrier flavour (FLAV) had moderate to strong effects on acceptability $\left[\eta_{p}{ }^{2}=.13\right.$ to .34 ], particularly on the measure of APP (Table 5.3). Savoury mealworm products were always rated more positively than sweet mealworm products [APP:+1.1, LIKE:+0.7, BUY:+0.5, TRY:+0.5] (Figure 5.3). A significant interaction effect $(p<.001)$ between VIS and FLAV was observed for all acceptability 
measures, where the visibility of mealworms within the product context resulted in a stronger negative effect when the carrier product was sweet, indicating that insect visibility may draw extra attention to the appropriateness of the combination.

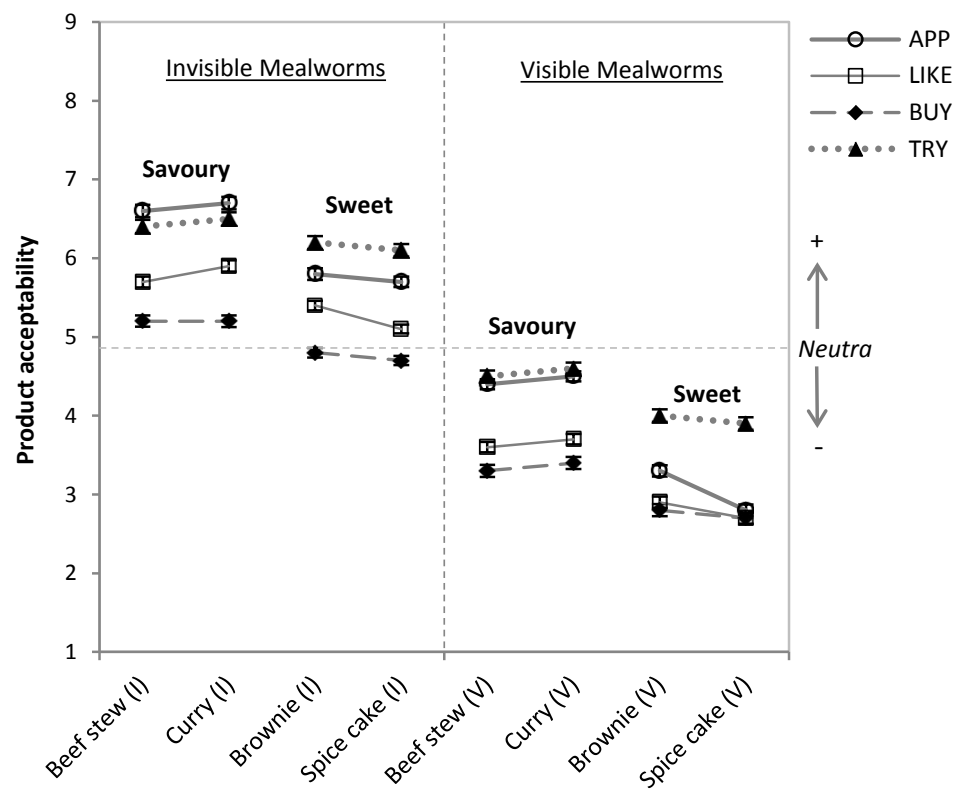

Figure 5.3. Mean acceptability ratings for mealworm products. (I)-Invisible mealworms, (V)-Visible mealworms. Error bars indicate standard errors.

The regression analysis (Table 5.2 ) showed that participants did not prefer product carriers of a certain origin (ORIG). Even though the ANOVA's (Table 5.3) showed significant effects for ORIG, the stronger interaction effects of FLAV*ORIG suggest that ORIG effects were specific to the carrier rather than its origin. The savoury Asian curry was preferred over the savoury Western beef stew, but the sweet Western brownie was preferred over the sweet Asian spice cake (Figure 5.3). Yet participants did report at the end of the survey that they would prefer Asian preparations to Western preparations of mealworms. This outcome suggests that participants may have preferences for carriers to be of a certain origin, but other product-related factors that were not accounted for in this study could play a more important role in determining the overall evaluations.

\subsubsection{Effects of familiarity and individual traits on acceptability}

Taste familiarity with the carrier products (TasCarrier) was not a significant predictor of acceptability (Table 5.2), but prior taste experience was related to higher ratings of Carrier-LIKE by 2.2 points $[t(1078)=38.89, p<.001]$. Levene's test indicated unequal variances $[F=34.72, p<.001]$, hence the degrees of freedom were adjusted from 7806 to 1078. The lack of effect of TasCarrier 
may be due to the high familiarity with the carrier products, where majority of the participants have tasted the carrier products before [beef stew: $92.6 \%$, curry: $91.6 \%$, brownie: $92.3 \%$, spice cake: $76.6 \%$, and are regular or occasional consumers of the products. In the context of this study, the effect of using a familiar carrier product was thus attributed to the sensory-liking of the carrier, rather than its familiarity per se.

The effects of the other significant factors related to familiarity and individual traits (TasMW, FNS, age, gender, education level) on the four acceptability measures were tested by ANOVA's and are reported in Table 5.4. Outcomes showed that factors related to taste familiarity and individual traits had relatively small effects on acceptability $\left[n_{p}{ }^{2} \leq .04\right]$. TasMW and FNS had a relatively stronger influence on acceptability ratings than the socio-demographic variables (age, gender, education level).

Taste familiarity with mealworms (TasMW) was generally low. Only $28.8 \%$ of the participants have tasted mealworms before, amongst whom experience was limited and consumption was not regular. However, there was a high awareness that mealworms can be used as food. Only $8.4 \%$ of the participants did not know about the edibility of mealworms despite most of them having never tasted mealworms before. TasMW had small but significant effects across all acceptability measures $\left[\eta_{p}{ }^{2}=.01\right.$ to .03] (Table 5.4), where prior taste experience with mealworms had a positive effect for each measure, with the greatest effect on TRY [APP: +0.9, LIKE: +1.1 , BUY: +1.3, TRY: +1.9] (Figure 5.4a). TasMW was related to MW-APP, where prior taste experience resulted in significantly higher ratings of MW-APP by 1.2 points $[t(782)=10.6, p<.001]$. Levene's test indicated unequal variances $[\mathrm{F}=81.86, \mathrm{p}<.001]$, hence the degrees of freedom were adjusted from 974 to 782. Overall, with effects of APP-MW accounted for, TasMW still had a significant impact on acceptability ratings (Table 5.2 ).

Table 5.4. Effects of familiarity and individual traits on acceptability of mealworm products.

\begin{tabular}{|c|c|c|c|c|c|c|c|c|}
\hline & \multicolumn{2}{|c|}{ APP } & \multicolumn{2}{|c|}{ LIKE } & \multicolumn{2}{|c|}{ BUY } & \multicolumn{2}{|c|}{ TRY } \\
\hline & $p$ & $\eta_{p}^{2}$ & $p$ & $\eta_{p}^{2}$ & $p$ & $\eta_{p}^{2}$ & $p$ & $\eta_{p}^{2}$ \\
\hline TasMW & $* * *$ & .01 & $* * *$ & .02 & $* * *$ & .02 & $* * *$ & .03 \\
\hline $\mathrm{FNS}^{\mathrm{A}}$ & $* * *$ & .01 & $* * *$ & .02 & $* * *$ & .03 & $* * *$ & .04 \\
\hline TasMW * FNS & $*$ & .00 & n.s. & .00 & $*$ & .00 & $* * *$ & .00 \\
\hline \multicolumn{9}{|l|}{ Covariates } \\
\hline Age & $* *$ & .00 & n.s. & .00 & n.s. & .00 & $* * *$ & .02 \\
\hline Gender & $* * *$ & .00 & $* * *$ & .01 & $* * *$ & .01 & $* * *$ & .02 \\
\hline Education level & n.s. & .00 & n.s. & .00 & n.s. & .00 & n.s. & .00 \\
\hline
\end{tabular}

Two-way ANOVA's performed on the main and interaction effects of TasMW and FNS levels, with socio-demographic variables as covariates. Significance indicated at $* p<0.05,{ }^{*} p<0.01, * * * p<0.001$, and n.s. indicates non-significant ( $p>0.05)$. AFNS levels (not scores) were used in this analysis.

Food neophobia scores (FNS) significantly influenced acceptability $\left[\eta_{p}^{2}=.01\right.$ to .04$]$, where higher FNS was significantly related to lower acceptability ratings (Table 5.2). FNS ranged from 10 to 55 
out of the possible range of 10 to $70[M=27.4, S D=8.8$, Cronbach's $\alpha=0.83]$, and were split into three groups with mean scores of $15.3(S D=2.2)$ for low-FNS ( $n=154), 26.9$ (SD=4.8) for midFNS ( $n=677)$, and $42.6(S D=4.4)$ for high-FNS ( $n=145)$ groups. The effects of FNS differed across acceptability measures (Figure 5.4b), where the low-FNS group were generally more positive in their ratings than the high-FNS group, but this difference was most pronounced for TRY [APP: +1.6, LIKE:+1.9, BUY: +2.2, TRY: 3.2].
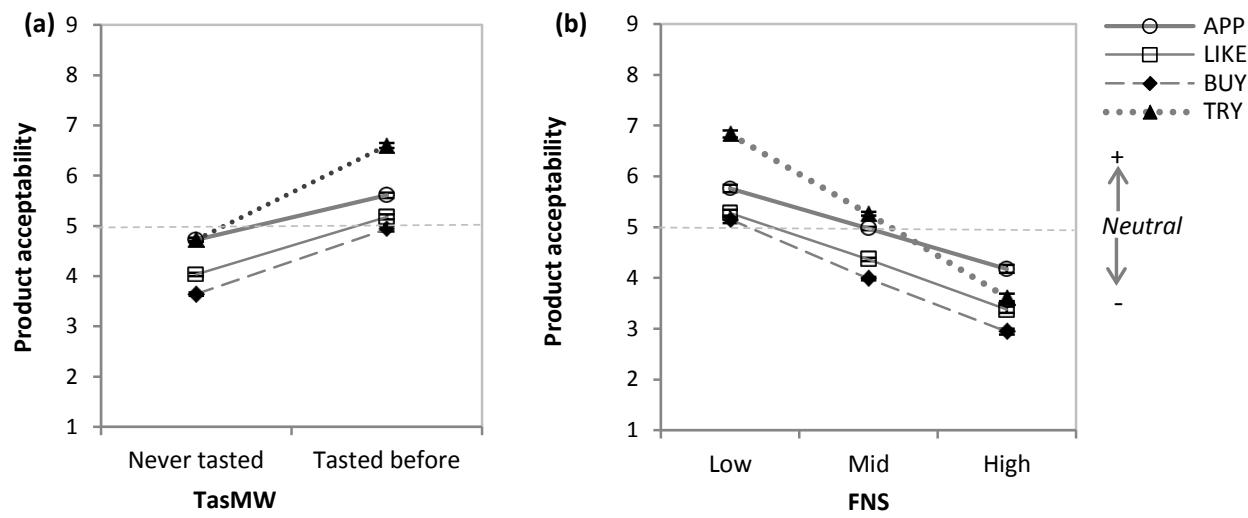

Figure 5.4. Mean product acceptability ratings according to (a) taste familiarity with mealworms, and (b) food neophobia. Error bars indicate standard errors.

In addition, more neophilic individuals (low-FNS) were more likely to have tasted mealworms before, where $51.9 \%$ of low-FNS ( $n=79), 28.0 \%$ of mid-FNS ( $n=187)$, and $9.8 \%$ of high-FNS ( $n=15)$ participants have done so. Both TasMW and FNS exerted separate influences on acceptability (Figure 5.5). High food neophobia scores resulted in lower acceptability ratings for both levels of TasMW, and prior taste experience with mealworms resulted in higher acceptability ratings for each level of FNS. Small but significant interactions between TasMW and FNS were observed for APP $\left[p<.05, \eta_{p}{ }^{2} \leq .01\right]$, BUY $\left[p<.05, \eta_{p}{ }^{2} \leq .01\right]$ and TRY $\left[p<.001, \eta_{p}{ }^{2} \leq .01\right]$, where mealworm taste experience had a stronger positive effect on more neophobic participants. Taste experience also reduced the differences in ratings between participants who differ in FNS, hence FNS plays a lesser role once a food becomes more familiar.

Neophilic (low-FNS) individuals tended to rate TRY positively regardless of whether they have tasted mealworms before, and those who have tasted mealworms before also tended to rate TRY positively regardless of their FNS levels (Figure 5.5). This was not the case for the other acceptability measures, where ratings tended to range from neutral to negative. Neophobic (high-FNS) individuals who have not tasted mealworms before were consistently negative in their ratings across all acceptability measures, which highlight the potential difficulties in introducing insect products to neophobic consumers for the first time. While FNS and TasMW had a strong impact on TRY, more limited effects on the other acceptability measures suggest that acceptance beyond the mere willingness to try is likely to be more challenging to achieve. 


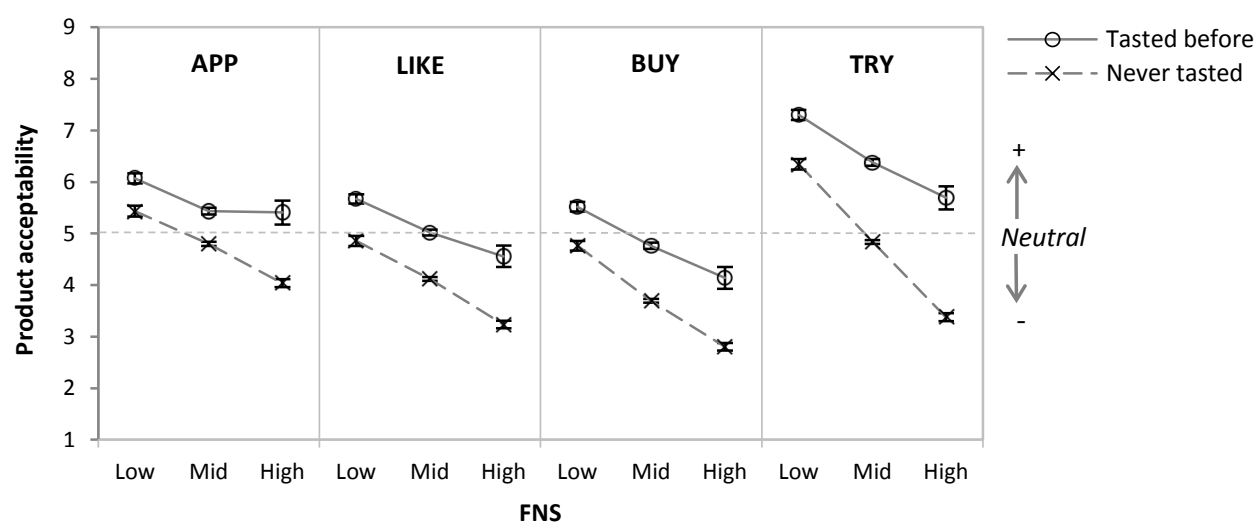

Figure 5.5. Interaction effects of food neophobia and mealworm taste familiarity on product acceptability ratings. Different letters indicate significant differences between food neophobia levels ( $p<0.05$, Bonferroni) and error bars indicate standard errors.

Socio-demographic variables played a minor role in predicting acceptability with small effects $\left[\eta_{p}{ }^{2} \leq .02\right]$ that were not always significant for the different acceptability measures (Table 5.4). Gender effects were significant across all acceptability measures, where male participants were consistently more positive than female participants in their evaluations. Gender effects were slightly larger for TRY $\left[\eta_{p}{ }^{2}=.02\right]$ than for the other acceptability measures $\left[\eta_{p}{ }^{2} \leq .01\right]$, where males could be particularly keen on trying in comparison to females, but not similarly optimistic on the other measures. On a similar note, younger participants rated TRY more positively than older participants $\left[\eta_{p}{ }^{2}=.02\right]$, but not the other measures. The regression analysis (Table 5.2) indicated that older participants were slightly more positive in LIKE and BUY, but age effects were not significant in the ANOVA's (Table 5.4). Overall, the effects of the socio-demographic variables on the acceptability measures were absent or small $\left[\eta_{p}{ }^{2} \leq .02\right]$, where mainly participants who are male and younger showing a particularly strong inclination to try the mealworm products, but not necessarily to buy them.

\subsection{DISCUSSION}

There is a growing number of studies on the acceptance of insects as food amongst Western consumers, but none have investigated in detail how different aspects of the product preparation influence hedonic evaluations and consumption intentions. This study used a set of insect-based products specifically designed to examine how the product preparation influences acceptability, and additionally investigated the effects of familiarity and individual traits, relative to the factors related to the product preparation. Here we looked beyond the mere willingness to try, and considered the potential for insect-based foods to be introduced on the Western market alongside other familiar foods. 
This study demonstrated that consumer acceptance is not simply achieved by preparing insects in familiar ways, which is often recommended in earlier research on novel foods (Pelchat \& Pliner, 1995; Wansink, 2002). While the higher sensory-liking of carrier products had a positive effect on the acceptability of the mealworm products, the addition of mealworms always resulted in a lower expected sensory-liking than the insect-free carrier products, even if the mealworms were not visible. This drop in sensory-liking was greater when carrier products were more liked. This raises questions about the viability of insect-based foods in Western markets as consumers often evaluate novel food products against what they are familiar with (Calantone et al., 2006). Using familiar preparations may lower barriers to acceptance due to the reduction of uncertainty and the positive sensory expectations with a familiar preparation method (Yeomans et al., 2008), but may result in greater product dissatisfaction when consumers evaluate novel insect-based products against the high expectations of product categories that they value.

The perceived appropriateness of mealworms as food had a much stronger influence on the acceptability of the mealworm products than the sensory-liking of the carrier product. This finding is in line with previous studies (Chapter 3 ) that showed that food appropriateness played a more important role than sensory-liking in the willingness to eat unusual foods like insects, where the mere belief of the presence of mealworms within a familiar carrier product (i.e. beef burger patties) lowered the expected sensory-liking and willingness to eat the product again after a positive taste experience. The present study further revealed that lower perceived food appropriateness of mealworms was linked to a greater drop in expected sensory-liking when mealworms are added into a product. One could thus expect that the effectiveness of using familiar preparations would be limited if the novel ingredient is not regarded to be appropriate for consumption. This is likely to be the case with insects in Western cultures where most consumers do not yet regard it as food (Looy et al., 2014; Yen, 2009). For such culturally-inappropriate foods, the prospect of consumption often evoke negative expectations regarding the product experience, and the product may be perceived to be contaminated by the insects even if their properties are not perceptible (Chapter 3; Rozin \& Fallon, 1987).

Moreover, the perceived appropriateness of the combination of mealworms and carrier products played an important role in determining product acceptability, where it was highly correlated with the drop in expected sensory-liking when mealworms are added to a familiar carrier product. Product appropriateness was also highly correlated with the expected sensoryliking, willingness to try and willingness to buy the mealworm products if priced equally with the carrier products. This implies that preparing insects in ways perceived to be incompatible could have strong negative effects on hedonic appraisals and subsequent intentions to consume. This is consistent with earlier observations (Stallberg-White \& Pliner, 1999) that showed that familiar preparations only improved the acceptability of a novel food when the combination is perceived to be appropriate. This demonstrates the importance of the contextual factors in 
food acceptance, where the use of foods in inappropriate ways could even result in liked foods being disliked (Cardello \& Schutz, 1996), not to mention novel foods like insects that are already negatively perceived by Western consumers.

Visibility of the mealworms within the carrier product was perceived to be highly inappropriate and resulted in low sensory-liking and consumption intentions. This is likely because the insect's appearance does not match Western expectations of how food looks like (Shelomi, 2015; Tucker, 2014). The visual features tend to bring up associations related to the insect's nature and origins when taste experience is lacking (Chapter 2), which are often negative due to its common image as pests and carriers of disease (Looy et al., 2014). One could thus speculate that the probability that Dutch consumers buy whole dried insects that are currently being sold in Dutch supermarkets is likely to be extremely low, even if consumers are provided with recipes that demonstrate how they could be easily incorporated into familiar foods (van Huis et al., 2012). Yet products containing insects in an invisible form might also not thrive on the market. In this study, participants expressed a high willingness to try if the mealworms were invisible, but were not similarly willing to buy the products. This finding was consistent with previous studies showing that curious consumers expressed a willingness to try both visibly and invisibly prepared insectbased foods, but had no intentions to consume them on a regular basis (Chapter 2; Tucker, 2014). Nevertheless, visibility is certainly important in the Western context as it could help to overcome the initial barriers of trial, but may become less important as taste experience increases. Consumers increasingly base their expectations on past sensory experiences rather than on the ideational associations with the insects (Chapter 2), it is therefore important to consider the sensory properties of the insect-based products during product development.

Clear flavour preferences were observed amongst participants, where the use of sweet carrier products was perceived to be inappropriate and resulted in lower acceptability ratings, even though the carrier product itself may be highly liked (e.g. brownie). Flavour principles usually develop due to repeated exposure to certain ways to certain ingredient combinations within the culture (Ahn et al., 2011). This study showed that despite their unfamiliarity with mealworms as food, the Dutch participants had defined expectations regarding how they should be appropriately prepared. This could be attributed to the marketing of insects as a sustainable meat alternative, which has created expectations of its use in similar ways to meat (Chapter 2). Currently, the use of insects as a meat substitute is the primary motivation for Western consumers to consider insects as food (Shelomi, 2015), and draws interest particularly from those who care about the environmental impact of food choice and who want to reduce their meat intake (Verbeke, 2015). Consumers may express a low interest in the regular consumption of an insect-based product if it does not fulfil these ethical motivations (Chapter 2). Hence, preparing insects in ways that match both flavour principles and consumer motivations would be critical to its acceptance. 
Familiarity and individual traits had a significant influence on acceptability, but played a relatively minor role in comparison to the product preparation. These factors had a stronger influence on the willingness to try than the other acceptability measures, where individuals differed much less in their evaluations of product appropriateness, expected sensory-liking and willingness to buy the mealworm products than they did in their expressed willingness to try. This is in line with observations of Chapter 2, where most participants expressed negativity and reluctance towards eating insects, but some were more willing to taste the insect-based products despite their negativity. Recognizing that the role of familiarity and individual traits differ between acceptability measures would have implications on research on novel food acceptance. Focussing on the initial willingness to try an unfamiliar product is likely to over-estimate the importance of these factors when assessing the long-term acceptance of the food.

Food neophobia and mealworm taste familiarity played a significant role in acceptability. Neophilics were always more positive than neophobics, and prior taste experience with mealworms resulted in more positive acceptability ratings. This confirms the results of earlier studies on this topic (Hartmann et al., 2015; Verbeke, 2015) and on other novel foods (Raudenbush \& Frank, 1999). Our findings additionally showed that these individuals tended to be highly positive about trying, but were not similarly optimistic with the other acceptability measures. Moreover, prior taste experience had a stronger positive effect on neophobic individuals. Differences due to food neophobia therefore become less relevant with increasing taste experience, up to the point where neophobics and neophilics generally do not differ in their evaluations of familiar foods (Raudenbush \& Frank, 1999; Tuorila et al., 2001). Even though neophobic individuals are less likely to taste novel foods, some neophobic individuals in this study have tasted mealworms before, which shows that neophobic individuals may still taste insect-based foods under certain circumstances. These outcomes suggest that low acceptance due to food neophobia and unfamiliarity with mealworms are likely to be reconciled through product trial. We thus recommend that efforts should be focussed more on creating products that lower the initial barriers of trying.

Lastly, socio-demographic factors influenced product acceptability but with generally small effects. Males were more positive than females, similar to the outcomes of previous studies (Chapter 3; Hartmann et al., 2015; Verbeke, 2015). This might relate to males generally being more adventurous than females and thus be more inclined towards tasting unusual foods (de Boer et al., 2013). Younger participants tended to be more willing to try, but not necessarily more willing to buy. This shows that they are more open to considering insects as food, but may be in it more for the thrill of the novel experience. Education level did not show clear effects on acceptability, similar to the findings of previous studies (Hartmann et al., 2015; Verbeke, 2015). 


\subsubsection{Implications on the consumer acceptance of insects as food}

The consumer acceptance of insects as food in the West is difficult to achieve. Reasons of sensory appeal and unfamiliarity are often given for the low acceptance of insects and other meat substitutes (Shelomi, 2015; Tucker, 2014). However, our findings showed that even if insects were prepared to resemble common foods, its perceived inappropriateness as food negatively impacts hedonic expectations and consumption intentions. The most ideal preparations of mealworms (i.e. invisible insect, appropriate flavour) were perceived to be inferior in taste to the original products, and willingness to buy was low even when priced equally to the original products. This may explain the failure of savoury ground insect products to gain acceptance in Belgium where stagnated sales resulted in their removal from supermarket shelves within two years, despite high initial interest from the public. At the moment, Western consumers generally do not see themselves consuming insects as a regular part of their diet (Tucker, 2014).

This raises the question of how insects could gain acceptance as food in the West. While creating appealing products strongly influences the acceptability of insect-based foods, long-term acceptance is harder to achieve if people are not used to the idea of eating insects. While taste exposure to insects and certain individual traits result in a greater openness to trying insect-based foods, the willingness to buy (this study) and consume as a regular part of the diet (Chapter 3) remains low. This is likely because numerous other factors such as cultural norms, deeply-rooted attitudes, practical issues, and knowledge gaps regarding the use of insects as food still stand in the way of its acceptance (Lensvelt \& Steenbekkers, 2014; Looy et al., 2014; Tan et al., 2015). The current low availability, high cost and lack of knowledge regarding the preparation and consumption of this new source of food still hinder the successful introduction of insects on the Western market (Shelomi, 2015). While it is possible to make good products and persuade people to try them, achieving consumer acceptance of insects as a regular food would certainly require larger efforts to address the socio-cultural and practical issues of food consumption.

The primary reason for Western consumers to consider insects as food is as a sustainable meat alternative (Shelomi, 2015), where promotional efforts have created interest in the use of insects as food despite the prevailing negativity. This works in the advantage of the introduction of insect-based foods, as the openness to trying creates an opportunity for perceptions of sensoryliking and food appropriateness to be gradually altered through taste exposure (Chapter 3 ). However, it is important to recognize that this initial willingness to try is not equivalent to long term acceptance, especially if sensory-liking is low compared to the food it substitutes.

In addition, ethical motivations for insect consumption seem to have created expectations regarding the use of insects as a meat substitute or at least in preparations that are also appropriate to meat (Chapter 2). This could be problematic as consumers generally expect meat substitutes to taste like meat (Hoek et al., 2011), which is difficult to accomplish when the properties of insects differ greatly from meat (Shelomi, 2015). Much work remains to be done 
to better understand how tasty insect-based products could be developed to fulfil consumer expectations. Introducing novel foods that have a strong negative image is certainly a challenge that requires not only changes in consumer perceptions, but also incentives to encourage consumption (Wansink, 2002). We suggest that the introduction of insects as food in the West should best be a combination of appropriate product design topped by efforts to address other barriers such as the current high price, low availability and poor image of insects as food in Western cultures (Shelomi, 2015).

\subsubsection{Limitations and future research}

A limitation of the study is that it was only based on labelled product images and did not involve actual tasting. The taste of mealworms is generally described as 'neutral' and 'nutty' (Gordon, 2013), which could be expected to have a sensory impact on products they are incorporated in. Consumers recognize the tastiness of the product after tasting it and tend to change their future consumption intentions according to how much they liked the consumption experience (Chapter 3). Future research should involve actual tasting and measures of perceived sensory properties, as sensory perceptions play an important role in food choice decisions once familiarity is gained (Martins \& Pliner, 2005). Further practical work to understand the properties of various edible species and their sensory qualities when processed differently would definitely aid in the development of tasty products. Nevertheless, this research showed that while product and individual factors are important determinants of consumer acceptance, factors related to the socio-cultural, economic and contextual factors still needs to be examined in greater depth. To provide better insights into the potential for insects to be introduced into the Western diet, future studies should extend beyond product evaluations, to examine how marketing, pricing, and various social and situational contexts affect purchase and consumption behaviours.

Recruitment through a university mailing list additionally raises the possibility that participants were unusually well informed about insects as food, as the university is very active in promotional and research activities on insects for human consumption. This was reflected in the relatively high awareness of mealworms as food, where only $8.4 \%$ of participants in this study did not know about mealworms as food, as opposed to $39.8 \%$ of participants in a study conducted at a different Dutch university (Chapter 3 ). Food neophobia scores of participants ranged from 10 to $55(M=27.4, S D=8.8)$, which is slightly more neophilic than reported in another Dutch study (Schickenberg et al., 2008), where scores ranged from 11 to 63 ( $M=30.1, S D=9.5)$. We expect that both higher awareness and lower food neophobia may contribute to a more open and positive attitude towards insects as food in comparison to the general Dutch population. Moreover, conclusions of this study were based on non-vegetarian participants. Vegetarians were excluded 
because the focus of the study was on the hedonic aspects of the combination, which may be biased by the rejection of mealworms and meat-based carriers due to specific dietary practices. Nevertheless, some vegetarians may actually be open to considering insects as food, as $28.8 \%$ claimed to have tasted insects before. They are a small but interesting group of consumers to study in future, and may have different expectations regarding their preferred ways of formulating products to fit their dietary habits.

\subsection{CONCLUSIONS}

The present study provided new insights into the influence of the product preparation, familiarity and individual traits on the acceptability of insect-based foods, and considered the potential for insects to gain acceptance amongst Western consumers. A high acceptability of insect-based products was not simply achieved by combining insects with familiar preparations, where acceptability depended very much on the perceived food appropriateness of the insect, and the perceived appropriateness of the combination. Insect-based products were expected to be inferior in taste in comparison to the original products, even if they were visually identical, which gives them a competitive disadvantage when placed on the market alongside existing products. Familiarity and individual traits significantly influenced acceptability ratings, but played a relatively minor role in comparison to the product-related factors. Individuals who were younger, male, more neophilic and who have tasted the insect before tended to be particularly willing to try but not similarly optimistic about buying. We conclude that for the introduction of insects as food in the West, it is important to design appropriate products. However, due to the low appropriateness of insects as food, there is a need for additional incentives to encourage consumption. We recommend that future research on the consumer acceptance of unusual novel foods like insects should look beyond the willingness to try, and place more emphasis on product design and alternative strategies for encouraging trial and repeat consumption.

\section{ACKNOWLEDGMENTS}

The authors thank Arnout Fischer for his advice on the study design and Yoeri Verbaan for his assistance with the product images and the preliminary surveys. 


\section{CHAPTER 6 ProOUCT's ROLE}

How will better products influence the sensory-liking

and willingness to buy insect-based foods?

Hui Shan Grace Tan

Yoeri Timothy Verbaan

Markus Stieger 


\section{ABSTRACT}

Insects have been established to be a more sustainable alternative source of protein in comparison to conventional meats, but have little appeal to those who are unfamiliar with their taste. Yet little attention has been given to understanding how more appealing products could be developed, and whether that is sufficient to encourage consumption of a culturally unusual food. By evaluating appropriate (i.e. meatball) and inappropriate (i.e. dairy drink) mealworm products along with the original mealworm-free products, this study provided new insights into how the product influences sensory-liking and willingness to buy insect-based foods for trial and regular consumption. Willing $(n=135)$ and unwilling tasters $(n=79)$ were recruited to explore differences between individuals who differ in their intentions to eat insects.

An appropriate product context improved the expected sensory-liking and willingness to buy mealworm products once and regularly. However, consumers should already be motivated to eat insects, otherwise the product type has little impact on consumption intentions. Descriptive sensory profiling revealed that mealworm products were expected and experienced to taste very different from the original mealworm-free products, but were generally preferred to taste similar to the original, albeit with some unique attributes. Using a familiar and liked product preparation could help to increase trial intentions, but the product should also be appropriate and taste good if it is to be regularly consumed.

We conclude that even with high interest and good products, willing consumers still hesitate to consume insect-based foods regularly due to other practical and socio-cultural factors. We recommend that future research should not only give emphasis to increasing initial motivations to try, but should address the barriers to buying and preparing insects for regular consumption, where issues relating to availability, pricing, knowledge and the social environment inhibit the uptake of this culturally new food. 


\subsection{INTRODUCTION}

Western interest in insects as food has escalated tremendously in recent years due to its nutritional and environmental advantages over conventional sources of protein (Boland et al., 2012; van Huis, 2013). Yet insects have little sensory appeal to most Western consumers (Deroy et al., 2015) and even the most motivated eaters remain unwilling to consume them on a regular basis after tasting the products (Chapter 2; Tucker, 2014). The low sensory appeal of insect-based foods poses difficulties for consumer acceptance as taste is an important driver of food choice (Steptoe et al., 1995). Few consumers are willing to compromise on taste for other desired benefits like health and sustainability, which offer less immediate and tangible benefits (Horgen \& Brownell, 2002; Tuorila \& Cardello, 2002; van Trijp \& Fischer, 2011). Improving the sensory experience of insect-based products is thus of critical importance if insects are to be eaten regularly. Studies on the consumer acceptance of insects as food in the West have so far given much attention to the psychological factors (e.g. food neophobia, risk perceptions) (Baker et al., 2016; Hartmann et al., 2015), but the role of the product preparation and its sensory qualities remains relatively unexplored. While it is known that a food's preparation could change its desirability by influencing its hedonic qualities and perceived appropriateness (Cardello \& Maller, 1982; Mela, 2001a), the cultural unfamiliarity and inappropriateness of insects as food (Chapter 3; Deroy et al., 2015) raises new questions regarding what consumers perceive to be appealing, and whether making better products could encourage regular consumption of this culturally unusual food.

Product-based strategies for improving the willingness to eat novel foods usually emphasize the use of familiar and liked flavours or preparations (Prescott et al., 2004; Tuorila, Andersson, et al., 1998). This helps by presenting the novel food in a way that looks and tastes familiar to consumers in order to create more positive expectations (Tuorila, Meiselman, Cardello, \& Lesher, 1998; Wansink, 2002). However, applying a liked flavour or preparation does not always increase the liking of a novel food (Pliner \& Stallberg-White, 2000; Stallberg-White \& Pliner, 1999). A usually liked food could also be disliked when presented in an inappropriate context or situation (Cardello et al., 2000; Schutz \& Martens, 2001). According to Schutz and Martens (2001), appropriateness is a measure of food acceptance that brings in the contextual aspects that are important to food acceptance, therefore enabling a better understanding and prediction of food behaviours.

Perceived appropriateness is often discussed in terms of the usage context (Cardello et al., 2000; Schutz \& Martens, 2001), which refers to whether the food product matches the situation or context of consumption. For instance, a delicious stew may not appeal at breakfast, or an insectbased food that is intended as a meat substitute may not be attractive as dessert (Chapter 2). This is based on the premise that consumers perceive products in terms of benefits, using them as a 
means to an end (Ratneshwar \& Shocker, 1991). The satisfaction delivered by a food product thus depends not only on the hedonic qualities of the food but also on the match between product category and usage situation. Perceived appropriateness could also refer to the food combination (Elzerman et al., 2011; Stallberg-White \& Pliner, 1999), in which case flavour principles are in check. For instance, using chocolate with grasshoppers may be seen to be an inappropriate combination with clashing flavours and textures (Chapter 2). In the present paper, we refer to the perceived appropriateness of the product preparation as 'product appropriateness'.

What is an appropriate way to prepare and use a food is very much influenced by individual experiences and cultural norms (Chapter 2; Rozin, 2006) where satisfactory past experiences are likely to influence what consumers deem to be appropriate (Giacalone \& Jaeger, 2016). For novel foods like insects that are culturally unfamiliar and never eaten by most Western consumers, expectations concerning how a food is appropriately used are likely to be less defined. At the moment, insects can be found in a wide variety of food applications in Western cookbooks (e.g. van Huis et al. (2012)), where insects seem to be used more for their protein than their sensory value. Thai consumers of insects, on the other hand, speak of specific ways of preparing and consuming various insect species, and are able to discuss extensively about the distinctive sensory qualities of each species and how they enhance the recipes they are combined with (Chapter 2). But even though culturally-defined rules may be lacking in Western cultures, savoury insect products are still perceived to be more appropriate than sweet products (Chapter 5) due to the primary positioning of insects as a meat alternative (Chapter 2; Shelomi, 2015). Consumers thus expect savoury insect products to taste better and are more willing to buy and try them (Chapter 5). It remains to be seen if products are also liked when they are tasted.

Given the unfamiliarity with insects as food, Western consumers tend to overlook their taste qualities (Deroy et al., 2015). There is a strong presumption that incorporating ground insects invisibly within food products would increase the acceptance of insect-based foods (Caparros Megido et al., 2014; Gmuer et al., 2016). However, it is possible that the insect-based foods are perceived instead to be inferior versions of the original products (Chapter 3 ), although the sensory-liking (i.e. hedonic appraisal of the sensory properties) has yet to be examined in detail. This study takes a closer look at what underlies the sensory-liking of insect-based products by examining the expected, experienced and ideal sensory properties of insect-based and insectfree products. Sensory research on a wide range of foods have shown that larger deviations of a food's sensory properties from the reported ideal properties negatively influences sensoryliking (Conner, 1994; Worch \& Punter, 2015). Yet for culturally unusual foods like insects, the perceived food appropriateness also plays an important role in acceptance and is strongly related to the sensory-liking of novel foods (Chapter 3). Rozin and Fallon (1987) define 'items that are not considered by a culture to be fit for consumption' to be inappropriate. For such foods the mere belief of its presence could already result in rejection (Chapter 3; Rozin \& Fallon, 1987), 
which could mean that certain consumers may dislike and decline to eat insects regardless of its product preparation and sensory qualities. To make a distinction from 'product appropriateness' that pertains to the product context, in this paper we refer to the perceived fitness of insects for human consumption as 'food appropriateness'.

The novelty and inappropriateness of insects as food adds further complexity to understanding its acceptance as food, especially in view of encouraging dietary change. First, consumers who are willing to try insects are not similarly willing to buy it (Chapter 5) or to use it as food on a regular basis (Tucker, 2014; Verbeke, 2015). Motivations for trial and regular consumption of insect-based foods are evidently very different, where repeated consumption is driven by more conventional considerations regarding food choice (e.g. taste, price) (House, 2016). Second, people differ in their willingness to adopt insects as food due to traits such as food neophobia and gender (Hartmann et al., 2015; Verbeke, 2015), but when specific products are evaluated, these individual factors seem to play a minor role in determining the acceptability of insectbased foods (Chapter 3 \& Chapter 5). The present study takes a closer look at these dimensions by exploring how willing and unwilling consumers differ in their evaluations and rationales regarding their willingness to buy insect-based foods for trial and regular consumption.

This study aims to gain a better understanding of how an appropriate product could influence the acceptance of a novel food like insects. Mealworms (Tenebrio molitor larvae) were selected for this study due to its recent availability as food in the Netherlands, such that findings have direct applications to the Dutch context. Using appropriate (i.e. meatball) and inappropriate (i.e. dairy drink) mealworm products, product influences on sensory-liking and willingness to buy mealworm products for trial and regular consumption were investigated. In addition, by comparing the evaluations and rationales given by willing and unwilling tasters, this study further explores the interplay between product and individual factors in the acceptance of a novel food. Implications of the study outcomes on product development and consumer acceptance of culturally novel foods with low sensory appeal are discussed.

\subsection{MATERIALS AND METHODS}

\subsubsection{Participants}

Participants were recruited from a previous survey-based study on Dutch consumers $(n=1057)$ (Chapter 5) during which they were invited to participate in a taste study involving mealworm products. A total of 596 positive responses and 296 negative responses were received, whereas the rest cited reasons of inconvenience and disinterest. Positive responders $(n=596)$ received a screening survey. Those who were between 18 to 65 years of age, had no allergies or dietary restrictions, and were available during study period were invited to participate in a sensory study. 
Negative responders $(n=296)$ were invited to participate in an online survey-based study that used the sensory questionnaire without the part that involved tasting products. A total of 135 willing tasters ( $33.0 \pm 16.7$ years, $80 \%$ female, $90.4 \%$ completed tertiary education) participated in the sensory study and 79 unwilling tasters $(50.9 \pm 21.2$ years, $65.8 \%$ female, $68.4 \%$ tertiary education) participated in the online study. A Wageningen University ethics committee granted ethical approval. Participants of the sensory study signed an informed consent form at the start of the study and received 10 euros reward upon completing the session. Participants of the online study were entered in a raffle for a chance to win one of three cash vouchers worth 20 euros.

\subsubsection{Selecting the product contexts}

Two products differing in perceived appropriateness as a preparation method for mealworms (Tenebrio molitor larvae) were used for this study. Previous studies revealed that Dutch consumers consider savoury mealworm products to be more appropriate than sweet ones due to the positioning of insects as a meat alternative (Chapter 2 \& Chapter 5). Product trials were performed to create various sweet and savoury mealworm products to identify products where the ground mealworms do not change the visual appearance of the original product, but create perceivable differences in flavour and texture. This was to prevent visual differences between mealworm and mealworm-free products from influencing the sensory perceptions. Based on the outcomes of the product trials, savoury meatballs and sweet dairy drink were selected. A survey with 64 Dutch persons confirmed that most considered meatballs to be an appropriate product for incorporating mealworms ( $n=50,78 \%$ ), and the dairy drink to be an inappropriate product for incorporating mealworms ( $n=48,75 \%)$.

\subsubsection{Product formulations}

Original meatballs used a basic recipe of $400 \mathrm{~g}$ of minced meat ( $50 \%$ beef, $50 \%$ pork), $40 \mathrm{~g}$ egg, $50 \mathrm{~g}$ breadcrumbs, $4 \mathrm{~g}$ salt and $0.8 \mathrm{~g}$ pepper. Mealworm meatballs were prepared by substituting $30 \%$ of the minced meat with ground mealworms. Mealworms were obtained blanched and frozen from Van de Ven (The Netherlands), a company that produces mealworms for human consumption. Original drink was a commercially available dairy breakfast drink 'Goedemorgen! Zuivelontbijt' from Friesland Campina (The Netherlands) that is a sweet strawberry-orange dairy drink with suspended cereal bits. Mealworm drink was prepared by adding $5 \%$ ground mealworms to the commercial drink. Mealworms were first lightly roasted on a pan for 3 minutes before grinding into a powder to be incorporated into the drink. Mealworm products differed from original products in flavour and texture but were similar in visual appearance. Images of the mealworms and products used in the study are shown in Figure 6.1. Due to visual similarity, the same meatball and drink images were presented for both original and mealworm versions of the products. 


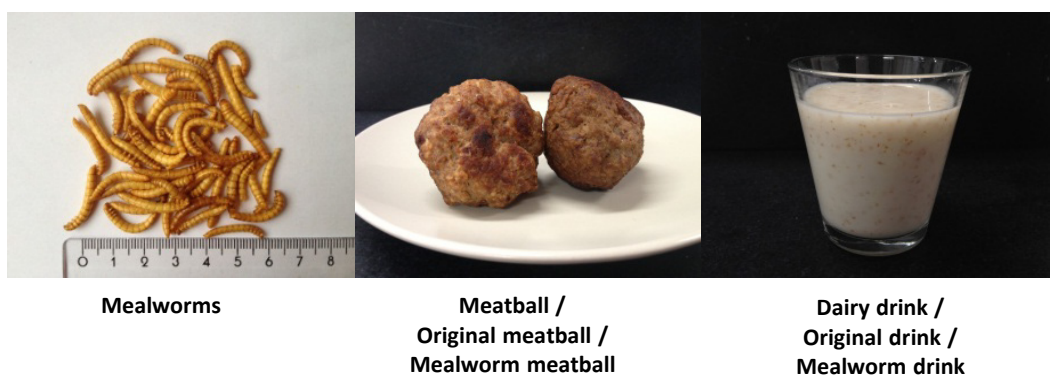

Figure 6.1. Images of mealworms, meatball and dairy drink products presented in the study.

\subsubsection{Sensory study}

\section{$\underline{6.2 .4 .1 . ~ S a m p l e ~ p r e p a r a t i o n ~}$}

Meatballs of 50g each were cooked on medium heat in a frying pan until browned on the surface, then simmered with water in a covered pan for 15 minutes until cooked through, then cooled and packed into aluminium boxes and stored at $-18^{\circ} \mathrm{C}$ in the week prior to the study. Samples were defrosted in the microwave at the start of each study day and kept chilled at $4^{\circ} \mathrm{C}$. Thirty minutes prior to serving, samples were heated on a hot plate until core temperature reached at least $70^{\circ} \mathrm{C}$. Samples were removed from the hot plate just before serving to participants, and placed on sample plates labelled with its identity (i.e. 'meatball without mealworms', 'meatball with mealworms'). Breakfast drinks were purchased from the supermarket in the week prior to the study and the mealworm drink was prepared at the start of each study day by dry roasting mealworms on a frying pan on medium heat for 3 minutes until slightly crispy, then blending it into a small amount of the commercial drink and diluting to $5 \%(\mathrm{w} / \mathrm{v})$ concentration. Drinks were kept chilled at $4^{\circ} \mathrm{C}$. To serve, $100 \mathrm{~g}$ drink was poured into labelled sample cups (i.e. 'breakfast drink without mealworms', 'breakfast drink with mealworms').

\section{$\underline{6.2 .4 .2 . ~ S e s s i o n ~ p r o c e d u r e s ~}$}

A single tasting session was conducted between 10.30 and $19.00 \mathrm{~h}$ over a period of two weeks in a consumer research room in Wageningen University. Partitions were placed on a long meeting table to create sensory booths. Upon arrival, participants filled in a consent form and were provided with water and crackers to clean their palate before and between tasting of each sample. Products were served one at a time and each session lasted about 45 minutes. Participants were instructed to eat as much as they wish. To control for contrast effects that may result from evaluating a more or less appealing product first, participants were assigned to receive one of four product presentation orders (meatball or drink first, and original or mealworm product first). Age, gender and prior taste experience with mealworms (recorded in the screening test) was balanced across groups that received different presentation orders. 


\subsubsection{Questionnaire design}

\section{$\underline{6.2 .5 .1 . ~ S e l e c t i o n ~ o f ~ s e n s o r y ~ a t t r i b u t e s ~}$}

Two lists of 24 sensory attributes (12 flavour, 12 texture) were created based on attributes used in a previous study (Chapter 3 ) and sample tasting performed by the research team, and refined by testing with 3 groups of Dutch university students. The preliminary list of attributes were presented to 6 Dutch university students who were asked to check the attributes that best described the 4 products before and after they tasted them, and to also check their ideal version of these products. Another group of 6 Dutch university students were asked to taste the products and generate their own flavour and texture attributes that describe the products. Sensory attributes that were not used or not easily understood were dropped and relevant attributes that were missing from the initial list were added. With the consolidated attributes, a pilot test was performed with third group of 6 Dutch students with the complete questionnaire. Final adjustments were made to remove irrelevant and add relevant attributes, and 2 lists (one for meatball and one for dairy drink) consisting of 24 sensory attributes each (12 flavour, 12 texture) were selected. Sensory attributes used for meatball evaluations were meaty, spiced, savoury, salty, onion, fatty, roasted, nutty, aftertaste, mealy, bland, musty, tender, juicy, firm, compact, soft, crusty, grainy, fibrous, dry, tough, mushy and slimy. Sensory attributes used for drink evaluations were fruity, yoghurt, sweet, milky, cereal, sour, aftertaste, savoury, roasted, mealy, musty, bitter, smooth, thick, fibrous, foamy, homogenous, heterogeneous, sticky, grainy, watery, crunchy, rough and dry/astringent.

\subsubsection{Questionnaire procedure}

The 4-part questionnaire (Figure 6.2) was designed in English and translated into Dutch by a native speaker for use in this study. The translation accuracy was checked using back-translation to English by another Dutch speaker. Willing tasters filled the full questionnaire during a sensory session, and unwilling tasters received an online questionnaire that excluded part 3 (product tasting). Participants evaluated meatball products first then drink products or in reverse (balanced design), and started with original product first then mealworm product or in reverse (balanced design). Product presentation orders were kept constant throughout the questionnaire.

In part 1, images of meatball, dairy drink and mealworms were shown with a short description of its composition and questions were asked about the product types and mealworm products in general. Familiarity with the taste ('How familiar are you with its taste?') was measured on a 9-point Likert scale anchored from 'totally unfamiliar' to 'extremely familiar', consumption frequency was measured with 6 categories (never tasted, tasted once, tasted several times, yearly, monthly, weekly), and sensory-liking of past or imagined taste experiences ('How tasty do you find its taste? If you have never tasted it before, how tasty do you expect it to be?') 
was measured on a 9-point Likert scale anchored from 'extremely not' to 'extremely'. Product appropriateness ('How appropriate is this product for introducing ground mealworms?') was measured on a 9-point Likert scale anchored from 'extremely inappropriate' to 'extremely appropriate'. Interest in trying mealworm products ('How interested are you to taste ground mealworm products once?') was measured on a 9-point Likert scale anchored from 'extremely not' to 'extremely', and willingness to buy mealworm products regularly ('How likely are you to buy ground mealworm products for regular consumption?') was measured on a 9-point Likert scale anchored from 'definitely not' to 'definitely'. Participants were then asked to write down their reasons for interest in trying and buying mealworm products.

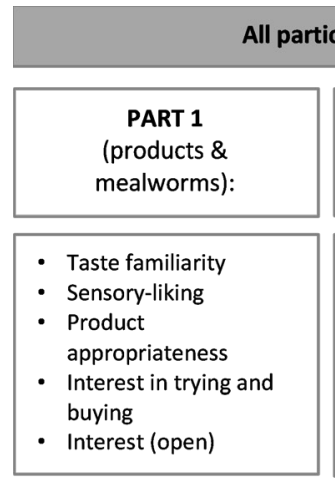

ll participants

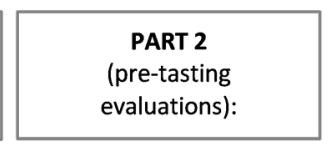

- Expected sensoryliking

- Expected sensory profile

- Ideal sensory profile

- Willingness to buy once and regularly
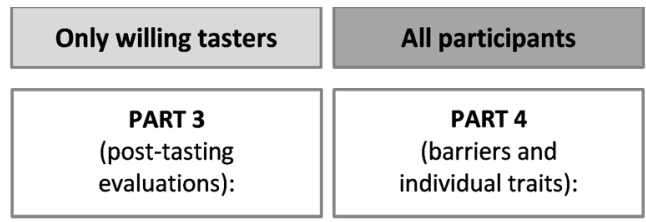

Figure 6.2. Questionnaire design.
- Experienced sensoryliking

- Experienced sensory profile

- Ideal sensory profile

- Willingness to buy once and regularly
- Food appropriateness

- Barriers to regular consumption (open)

- Food neophobia

- Socio-demographic factors

Parts 2 and 3 involved the evaluations of the original and mealworm products. In part 2, product images were displayed and participants were instructed to imagine tasting the specific original and mealworm products and evaluate accordingly. Expected sensory-liking ('How tasty do you expect it to be?') was first measured for each product on a 9-point Likert anchored from 'extremely not' to 'extremely'. Participants then evaluated meatball and drink products based on flavour and texture using Check-All-That-Applies (CATA) checklists that can be reliably used for sensory profiling with untrained consumers (Jaeger \& Ares, 2014; Varela \& Ares, 2012). Flavour and texture attributes were randomized within their respective tables and presented in a fixed order for each participant. After filling in each expected sensory profile, participants were asked to complete the same CATA question for their ideal product (Bruzzone et al., 2012; Ruark, Vingerhoeds, Kremer, Nijenhuisde Vries, \& Piqueras-Fiszman, 2016). Participants were then asked to imagine the mealworm products in the shop and willingness to buy once ('How likely are you to buy it to taste once?') and regularly ('How likely are you to buy it for regular consumption?') was measured on 9-point Likert scales anchored from 'definitely not' to 'definitely'. In part 3, participants were instructed to taste and evaluate products as they were served to them one after the other. For each product, the experienced sensory-liking ('How tasty do you experience it to be?'), experienced sensory profile, 
ideal sensory profile and willingness to buy once and regularly were measured in the same way as in part 2. Only willing tasters answered part 3 of the questionnaire.

In part 4, food appropriateness of mealworms ('How appropriate as food do you consider it to be?') was measured on 9-point Likert scales anchored from 'extremely inappropriate' to 'extremely appropriate' (Chapter 3). Participants were asked to write down their reasons for not consuming insects regularly. Food neophobia was measured with a Dutch-translated version of the Food Neophobia Scale (FNS) (Pliner \& Hobden, 1992), which was previously performed by a professional translation agency using a translation and back-translation procedure (Hoek et al., 2011), Participants also reported their gender, age, gender, highest education completed, and affiliation with Wageningen University.

\subsubsection{Data analysis}

Responses on familiarity, product and food appropriateness, expected and experienced sensory-liking, consumption interest, and willingness to buy were transformed into numbers (1-9). Consumption frequencies of 'never tasted', 'tasted once' and 'tasted several times' were considered to be consumption that is not regular, and 'yearly', 'monthly' and 'weekly' consumption were considered to be regular. Ratings of FNS were tabulated according to Pliner and Hobden (1992). Pearson's product moment correlations were used to analyse the relations between measures. Differences between responses of willing and unwilling tasters were analysed with independent samples t-tests. Mealworm and product type effects on sensory-liking of products were analysed using repeated measures two-way analysis of variance (ANOVA). Buying frequency and product type effects on willingness to buy mealworm products was analysed using repeated measures two-way ANOVA. Bonferroni corrections were applied to all post-hoc tests with a level of significance of 0.05 . Changes in sensory-liking and willingness to buy upon tasting was further analysed using paired samples t-tests.

Hierarchical linear regressions were performed to assess the predictive effects of the product type, food appropriateness of mealworms, and sensory-liking on the willingness to buy the mealworm products once and regularly. Analysis of CATA results was based on frequencies of checks and Cochran's $Q$ tests were performed to test for significant differences between frequency data for each sensory attribute across products. Correspondence analysis was performed to visualise how products are relatively positioned, and to explore differences between the sensory perceptions of willing and unwilling tasters. Responses to open questions relating to the interest and barriers of consumption were content-analysed by two researchers. Codes were categorized according to sensory-, product-, individual- and context-related reasons. Each category was further subdivided into positive, negative, and neutral (i.e. statements of criteria) codes and frequencies were tabulated. 


\subsection{RESULTS}

\subsubsection{Differences between willing and unwilling tasters}

\subsubsection{Differences in participant characteristics}

Willing tasters were on average younger ( $33.0 \pm 16.7$ years vs $50.9 \pm 21.2$ years), comprised of more females ( $80 \%$ vs $66 \%$ ), and were more likely to have already completed tertiary education (90\% vs $68 \%$ ). These characteristics were very much influenced by the large proportion of subjects who were affiliated to Wageningen University (i.e. students and employees) taking part in the sensory study, which may be due to interest and convenience. Food neophobia scores (FNS) determined based on the scale developed by Pliner and Hobden (1992) ranged from 10 to 61 and were significantly lower for willing tasters $(M=22.1, S D=5.9)$, than for unwilling tasters $(\mathrm{M}=32.6, \mathrm{SD}=9.9)[\mathrm{t}(212)=9.71, \mathrm{p}<.001$, Cronbach's $\alpha=0.85]$. According to the food neophobia levels (low-FNS:<19, mid-FNS:19 to 36 , high-FNS:>36) used in the previous study from which participants of the present study were recruited (Chapter 6), willing tasters were either mid- or low-FNS, whereas unwilling tasters were from all three FNS categories, indicating that neophilic consumers could also be unwilling to eat insects.

\subsubsection{Evaluations of mealworms as food}

Figure 6.3 shows that participants were generally unfamiliar with the taste of mealworms.

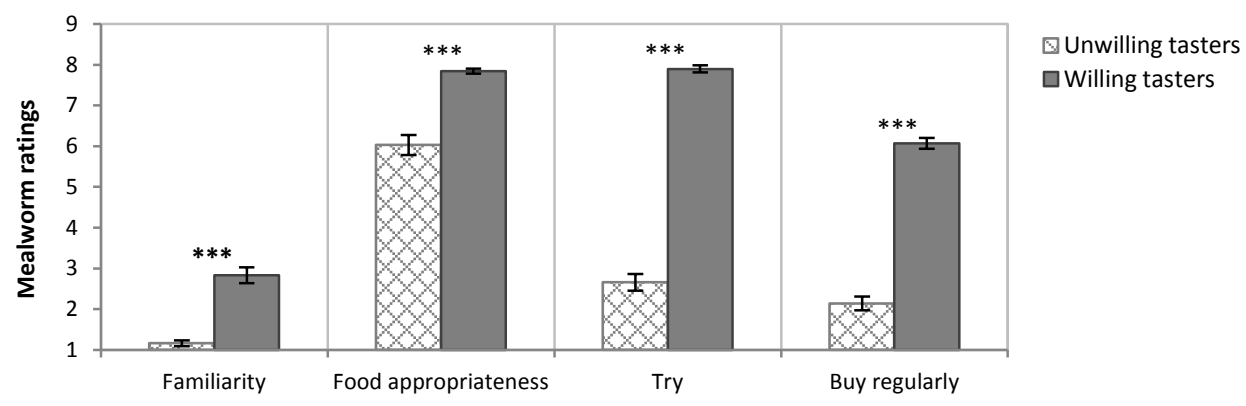

Figure 6.3. Mean taste familiarity, food appropriateness and consumption intentions of mealworms by unwilling and willing tasters. Significant differences between participant groups indicated by ${ }^{* * *} p<0.001$ (Bonferroni). Error bars indicate standard error.

Willing tasters were slightly more familiar with its taste than the unwilling tasters $[t(212)=6.44$, $\mathrm{p}<.001]$. Based on the reported consumption frequency, more of the willing tasters than the unwilling tasters have tasted mealworms before [52.6\% vs $10.1 \%$ ]. Willing tasters also perceived mealworms to be higher in food appropriateness, whereas unwilling tasters gave significantly 
lower ratings $[\mathrm{t}(212)=-8.96, \mathrm{p}<.001]$. Willing tasters were very positive in their intentions to try mealworm products but less so in their intentions to buy regularly $[t(134)=14.84, p<.001]$, whereas unwilling tasters were only slightly more willing to try than to buy mealworm-based products $[t(78)=3.93, p<.001]$. Unwilling tasters were both very unwilling to try or buy mealworm products regularly, and were much less positive than the willing tasters on both measures [try: $t(212)=-27.54, p<.001$; buy regularly: $t(212)=-17.98, p<.001]$.

\section{$\underline{6.3 .1 .3 .}$ Evaluations of meatball and dairy drink products}

Figure 6.4 shows participants' taste familiarity, sensory-liking and product appropriateness (i.e. perceived appropriateness as a preparation method for mealworms) of meatball and dairy drink products.
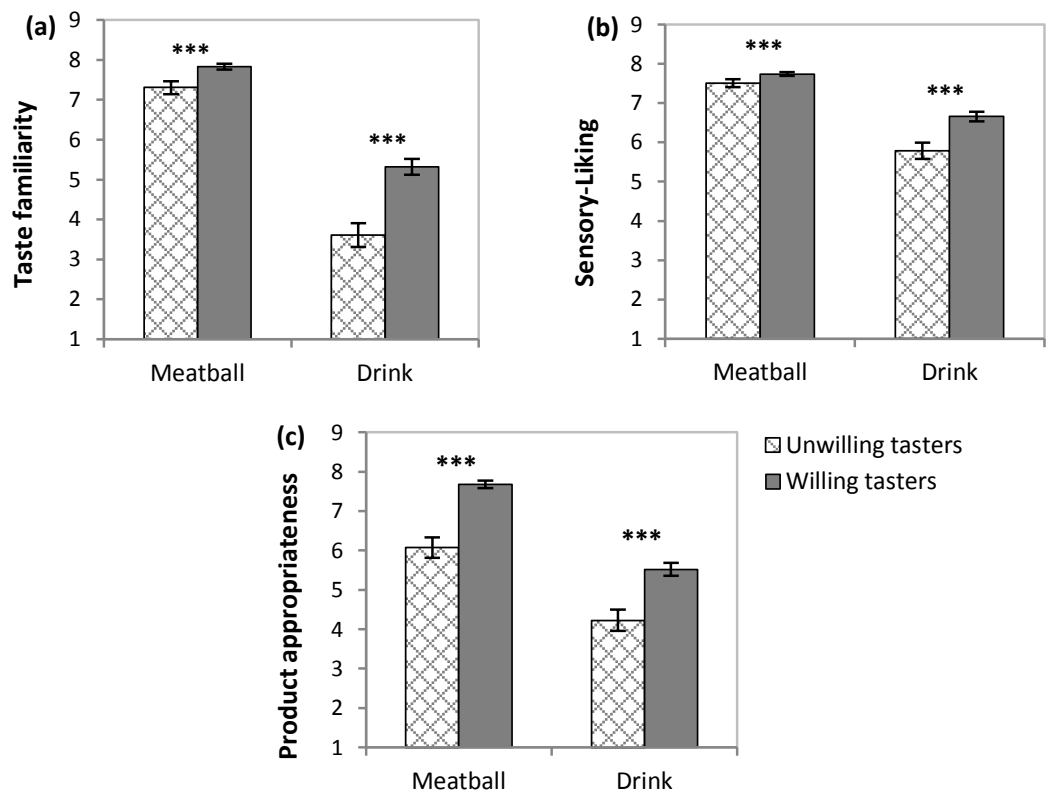

$\square$ Unwilling tasters $\square$ Willing tasters

Figure 6.4. Mean (a) taste familiarity, (b) sensory-liking and (c) product appropriateness of meatball and dairy drink by unwilling and willing tasters. Significant differences between participant groups are indicated by $* * * \mathrm{p}<0.001$ (Bonferroni). Error bars indicate standard error.

Meatballs were highly familiar in taste for both willing and unwilling tasters. Based on the reported consumption frequency, most participants are regular consumers of meatballs [97.8\% of willing tasters; $96.2 \%$ of unwilling tasters]. The taste of the dairy drink was less familiar for both willing $[t(134)=13.01, p<.001]$ and unwilling $[t(78)=11.84, p<.001]$ tasters (Figure 6.4a). Based on the reported consumption frequency, less than half of the participants were regular consumers of the dairy drink [ $45.2 \%$ of willing tasters; $19.0 \%$ of unwilling tasters]. However, $89.8 \%$ of willing tasters and $59.5 \%$ of unwilling tasters have at least tasted the drink before. Willing tasters were 
more positive in their evaluations of sensory-liking (Figure 6.4b) and product appropriateness (Figure 6.4c) than unwilling tasters $(p<.001)$. For both participant groups, meatballs were higher in sensory-liking [willing: $\mathrm{t}(134)=8.69, \mathrm{p}<.001$; unwilling: $\mathrm{t}(78)=7.73, \mathrm{p}<.001$ ], and product appropriateness [willing: $\mathrm{t}(134)=11.76, \mathrm{p}<.001$; and unwilling: $\mathrm{t}(78)=6.62, \mathrm{p}<.001$ ]. Product appropriateness was weakly correlated with the sensory-liking $[r=+.28, p<.001]$ and familiarity with its taste [Pearson's $r=+.33, p<.001$ ], whereas sensory-liking and familiarity were strongly correlated [Pearson's $r=+.60, p<.001]$.

\subsubsection{Sensory-liking of mealworm products}

\subsubsection{Product influences on sensory-liking}

Before tasting, expected sensory-liking of mealworm products was strongly correlated with product appropriateness for both willing [Pearson's $r=+.59, p<.001$ ] and unwilling tasters [Pearson's r =+.49, p<.001]. From Table 6.1 and Figure 6.5, it can be observed that the mealworm's presence had a much more negative impact on the expected sensory-liking of products for the unwilling tasters [meatball:-3.0; drink:-2.8] than the willing tasters [meatball:-0.6; drink:-1.0]. Meatball products were generally rated by both participant groups to be higher in expected sensory-liking than the drink products (Figure 6.5). For the willing tasters, the negative effect of the mealworm presence was significantly lessened when mealworms were prepared in the more appropriate meatball product, as shown by the significant interaction effect (Table 6.1).

Table 6.1. Two-way repeated measures ANOVA's of main and interaction effects of product type and mealworm presence on the sensory-liking of products.

\begin{tabular}{|c|c|c|c|c|c|c|c|c|c|}
\hline & \multirow{2}{*}{\multicolumn{3}{|c|}{$\begin{array}{c}\text { Unwilling Tasters }(\mathbf{n}=\mathbf{7 9}) \\
\text { Before Tasting }\end{array}$}} & \multicolumn{6}{|c|}{ Willing Tasters ( $\mathrm{n}=135)$} \\
\hline & & & & \multicolumn{3}{|c|}{ Before Tasting } & \multicolumn{3}{|c|}{ After Tasting } \\
\hline & $F_{(1,78)}$ & $p$ & $\eta_{p}{ }^{2}$ & $F_{(1,134)}$ & $\mathrm{p}$ & $\eta_{p}^{2}$ & $F_{(1,134)}$ & $\mathrm{p}$ & $\eta_{p}^{2}$ \\
\hline Product type (P) & 38.20 & $<.001$ & .33 & 105.32 & $<.001$ & .44 & 2.50 & .117 & .02 \\
\hline Mealworm (Mw) & 235.27 & $<.001$ & .75 & 87.83 & $<.001$ & .40 & 15.37 & $<.001$ & .10 \\
\hline $\mathrm{P}^{*} \mathrm{Mw}$ interaction & 0.49 & .488 & .01 & 14.60 & $<.001$ & .10 & 3.47 & .065 & .03 \\
\hline
\end{tabular}

Bold highlights indicate significant values.

After tasting (willing tasters), the experienced sensory-liking was no longer significantly correlated with product appropriateness [Pearson's $r=+.08, p>.05$ ]. Table 6.1 and Figure 6.5 show that experienced sensory-liking of the mealworm products did not differ significantly, but mealworm products were rated significantly lower than the original products. The taste experience reduced the negative impact of the mealworms on the drink [-0.3] but not the meatball [-0.6], where the mealworm drink was perceived to be significantly better than expected $[t(134)=5.14$, $p<.001]$ while the original drink was as expected $[t(134)=0.71, p=.482]$, and the original meatball $[t(134)=-5.15, p<.001]$ and mealworm meatball $[t(134)=-3.58, p<.001]$ were perceived to be worse than expected. The following section (6.3.2.2) examines the underlying sensory perceptions. 


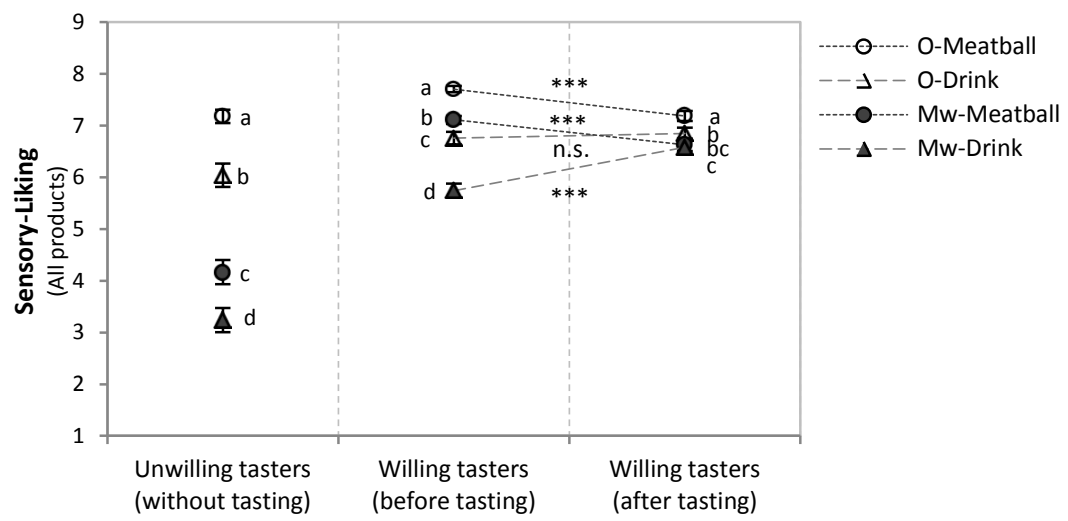

Figure 6.5. Mean sensory-liking of original and mealworm meatballs and drinks by unwilling tasters (without tasting) and willing tasters (before and after tasting). Significant differences ( $p<0.05$, Bonferroni) between products are indicated with different letters. Significant differences between before and after tasting ratings are indicated by $* * * p<0.001$ and $n$.s. for not significant $(p>0.05)$. Error bars indicate standard errors.

\subsubsection{Sensory perceptions of mealworm products}

Correspondence analysis (CA) plots were based on the CATA attribute frequencies by willing tasters (W) and unwilling tasters $(\mathrm{U})$ and shown for meatball (Figure 6.6a) and dairy drink (Figure 6.6b) products. Frequency data and outcomes of Cochran's $Q$ test were used for the interpretations of the CA plots but are not shown here. Mealworm products were evaluated to be different from original products on multiple attributes both before and after tasting. The addition of mealworms to the products resulted in sensory qualities that seemed less desirable for both meatballs and dairy drinks. Based on the evaluations of the willing tasters, the mealworm meatball was expected to be significantly $(p<.05)$ more musty, mealy, nutty, dry, fibrous and grainy, and significantly $(p<.05)$ less meaty, fatty and juicy than the original meatball. The mealworm drink was expected to be significantly $(p<.05)$ more musty, mealy, roasted, savoury, dry/astringent, rough, crunchy and grainy, and significantly $(\mathrm{p}<.05)$ less fruity, sweet and smooth than the original drink. Willing and unwilling tasters were quite similar in their expectations of the original products, whereas stronger mealworm-related properties (i.e. mealy and musty) were anticipated by the unwilling tasters than the willing tasters (see dotted arrows in Figure 6.6).

The ideal mealworm product was very similar to the ideal original product, but differing $(p<.05)$ on 2 attributes (nutty, onion) for the meatballs, and 1 attribute (mealy) for the drink. Ideal sensory profiles were evaluated both before and after tasting to explore if participants change their minds regarding the ideal products after experiencing its properties because of their unfamiliarity with its taste, where we speculated that any positive attributes contributed by the addition of mealworms may possibly be incorporated into the ideal profiles after tasting. However, results from Cochran's $Q$ test showed that ideal profiles did not significantly change after the taste experience. 
a) Meatball products

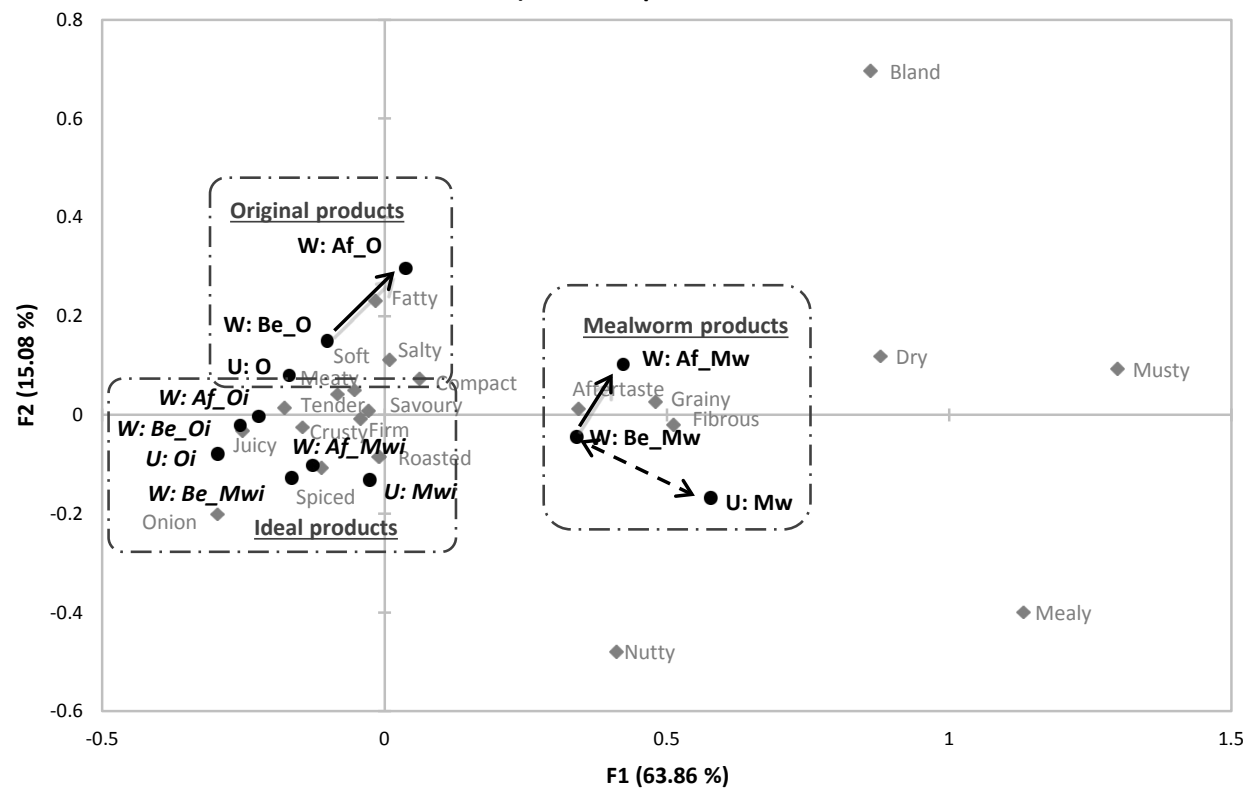

b) Dairy drink products

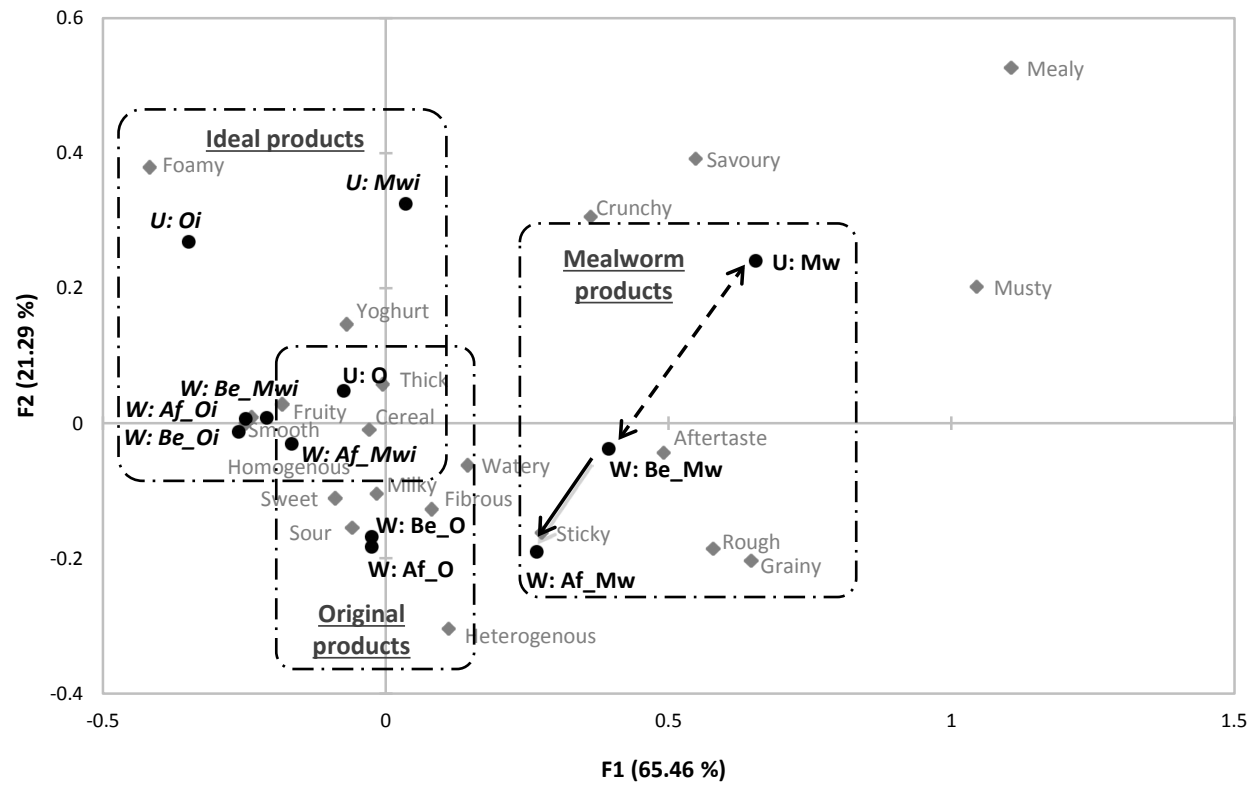

Figure 6.6. Correspondence analysis bi-plots of CATA frequencies for sensory perceptions for (a) meatball and (b) dairy drink products. Original (O) and mealworm (Mw) products for unwilling (UW) and willing (W) tasters, before (Be_) and after (Af_) tasting indicated for willing tasters, and ideal products (i) are shown. Dotted arrows indicate differences between willing and unwilling tasters for the mealworm products, and solid arrows indicate the sensory change upon tasting. 
From Figures $6.6 \mathrm{a}$ and $\mathbf{6 . 6 \mathrm { b }}$, it can be observed that mealworm products deviated more from their ideal profiles than the original products, both before and after tasting. After tasting the products (willing tasters), many of the expected differences between the original and mealworm meatballs were perceived, with the addition of an experienced aftertaste and that the mealworm meatballs were not more grainy and fibrous. The mealworm drink was more similar to the original drink than expected, where it was not perceived to be less fruity and smooth, or more savoury, roasted, crunchy and dry/astringent, but was unexpectedly less thick. Mealworm drink was perceived to be more similar to the original drink after tasting, but this was not the case for the mealworm meatball.

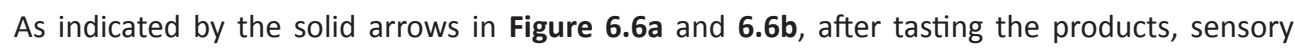
profiles of the original and mealworm meatballs shifted away from their ideals (i.e. less ideal), but did not shift for the original drink (i.e. no change) and shifted closer for the mealworm drink (i.e. more ideal). These observations corresponded with the changes in sensory-liking scores in the previous section (6.3.2.1), where the larger and smaller deviations from ideal corresponded with increases and decreases in sensory-liking upon tasting the products. These outcomes suggest that mealworm products that tasted more similar to the ideal product were more liked.

\subsubsection{Willingness to buy mealworm products once and regularly}

\subsubsection{Product influence on willingness to buy}

Before tasting, product appropriateness was positively correlated with willingness to buy mealworm products for both willing [once: Pearson's $r=+.36, p<.001$, regularly: Pearson's $r=+.44$, $\mathrm{p}<.001$ ] and unwilling tasters [once: Pearson's $r=+.18, p=.028$, regularly: Pearson's $r=+.22$, $\mathrm{p}=.006]$. After tasting (willing tasters), product appropriateness was less strongly correlated with the willingness to buy mealworm products [once: Pearson's $r=+.22, p<.001$, regularly: Pearson's $r=+.26, p<.001$ ]. From Figure 6.7 it can be seen that, unlike the willing tasters, unwilling tasters showed very low willingness to buy mealworm products. While the more appropriate mealworm meatball was rated significantly higher in willingness to buy than the less appropriate mealworm drink, the product type had a stronger influence on willingness to buy for the willing tasters than for the unwilling tasters (Table 6.2).

After tasting (willing tasters), product appropriateness was less strongly correlated with the willingness to buy mealworm products [once: Pearson's $r=+.22, p<.001$, regularly: Pearson's $r=+.26, p<.001]$. The sensory experience had a greater negative impact on the willingness to buy the mealworm meatball although it was initially perceived to be more appropriate (Figure 6.7). There was a significant decrease in the willingness to buy the mealworm meatball by 0.9 points $[t(134)=-4.59, p<.001]$ and mealworm drink by 0.5 points $[t(134)=-2.19, p=.031]$ for trial consumption. However, willingness to buy the mealworm meatball was always higher than the mealworm drink, despite both products being similar in experienced sensory-liking (see 
section 3.2.1). Willingness to buy regularly also decreased significantly for the mealworm meatball by 0.6 points $[t(134)=-2.88, p=.005]$, but did not change for the mealworm drink $[t(134)=+0.17$, $\mathrm{p}=.867]$, even though sensory-liking of the mealworm drink increased after tasting (see section 6.3.2.1). Overall, willingness to buy once always decreased after tasting, and willingness to buy regularly was always lower than the willingness to buy once for reasons beyond that of sensoryliking.

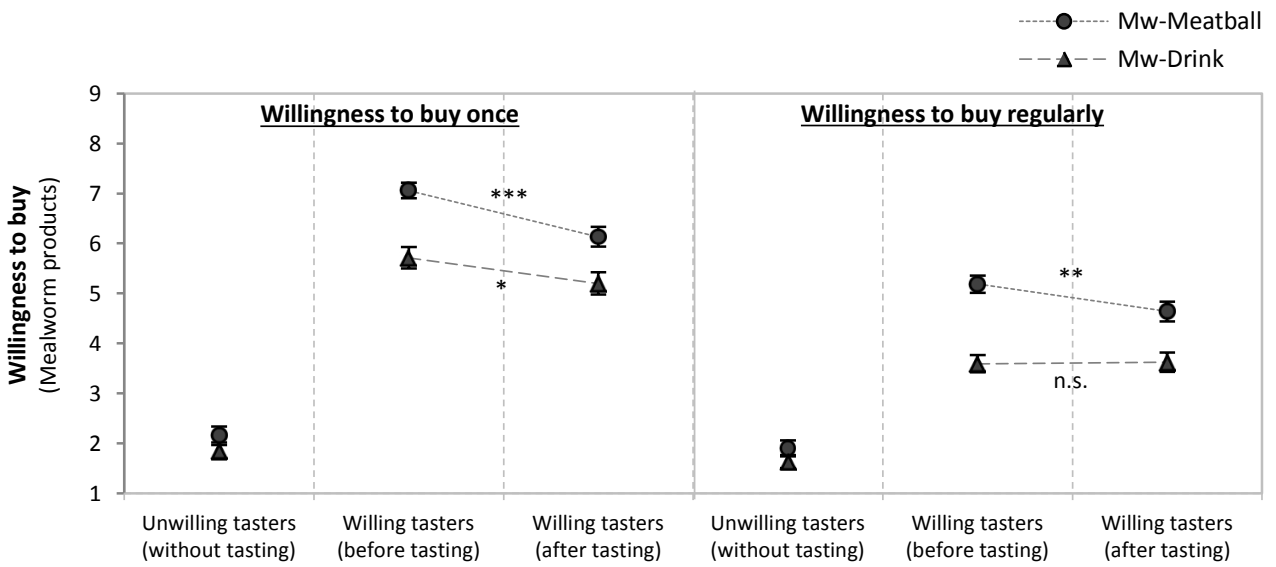

Figure 6.7. Mean willingness to buy mealworm products once and regularly by unwilling tasters (without tasting) and willing tasters (before and after tasting). Significant differences are indicated by different letters ( $p$ b 0.05 , Bonferroni). Significant differences between before and after tasting ratings are indicated by $* * * p<0.001, * * p<$ $0.01, p<0.05$ and n.s. for not significant $(p>0.05)$. Error bars indicate standard errors.

Table 6.2. Two-way repeated measures ANOVA's of main and interaction effects of product type and consumption frequency on the willingness to buy mealworm products.

\begin{tabular}{|c|c|c|c|c|c|c|c|c|c|}
\hline & \multirow{2}{*}{\multicolumn{3}{|c|}{$\begin{array}{c}\text { Unwilling Tasters }(\mathbf{n}=\mathbf{7 9}) \\
\text { Before Tasting }\end{array}$}} & \multicolumn{6}{|c|}{ Willing Tasters ( $n=135)$} \\
\hline & & & & \multicolumn{3}{|c|}{ Before Tasting } & \multicolumn{3}{|c|}{ After Tasting } \\
\hline & $F_{(1,78)}$ & $p$ & $\eta_{p}^{2}$ & $F_{(1,134)}$ & $\mathrm{p}$ & $\eta_{p}^{2}$ & $F_{(1,134)}$ & $\mathrm{p}$ & $\eta_{p}^{2}$ \\
\hline Product type (P) & 5.38 & .023 & .06 & 79.84 & $<.001$ & .37 & 17.36 & $<.001$ & .12 \\
\hline Frequency (F) & 14.75 & $<.001$ & .16 & 273.34 & $<.001$ & .67 & 218.25 & $<.001$ & .62 \\
\hline $\mathrm{P} * \mathrm{~F}$ interaction & 0.17 & .683 & .00 & 2.82 & .095 & .02 & 0.26 & .609 & .00 \\
\hline
\end{tabular}

Bold highlights indicate significant values.

\subsubsection{Predicting the willingness to buy mealworm products}

Hierarchical linear regression analyses were conducted to examine how the product type, food appropriateness of mealworms and sensory-liking of the mealworm products predict the willingness to buy mealworm products once and regularly before and after the products were tasted (Table 6.3). Unwilling tasters were excluded from this analysis as they had little intention to consume mealworm products. Individual characteristics (e.g. FNS, age, gender) did not have significant predictive value and were also excluded from the analysis. 
Table 6.3. Predictions of willingness to buy mealworm products once and regularly.

\begin{tabular}{|c|c|c|c|c|}
\hline \multirow{2}{*}{$\begin{array}{l}\text { Willingness to buy } \\
\text { mealworm products: }\end{array}$} & \multicolumn{2}{|c|}{ Before Tasting } & \multicolumn{2}{|c|}{ After Tasting } \\
\hline & Once & Regularly & Once & Regularly \\
\hline \multicolumn{5}{|l|}{ Product type (Std. $\beta$ ) } \\
\hline Product appropriateness & +0.08 & $+0.21 * * *$ & +0.09 & $+0.15^{* *}$ \\
\hline Product familiarity & $+0.13^{*}$ & $+0.18^{* *}$ & $+0.23 * * *$ & $+0.14^{*}$ \\
\hline Product sensory-liking & $+0.16^{*}$ & $+0.18^{* *}$ & -0.00 & +0.06 \\
\hline \multicolumn{5}{|l|}{ Mealworms (Std. $\beta$ ) } \\
\hline Food appropriateness & $+0.25^{* * *}$ & $+0.12^{*}$ & $+0.18 * * *$ & $+0.18 * * *$ \\
\hline \multicolumn{5}{|l|}{ Mealworm product (Std. $\beta$ ) } \\
\hline Sensory-liking & $+0.29 * * *$ & $+0.17^{*}$ & $+0.50 * * *$ & $+0.44 * * *$ \\
\hline \multicolumn{5}{|l|}{ Model parameters } \\
\hline$\overline{\text { Product type }\left(\Delta \mathrm{R}^{2}\right)}$ & $+0.27^{* * *}$ & $+0.33^{* * *}$ & $+0.13^{* * *}$ & $+0.12^{* * *}$ \\
\hline Mealworms $\left(\Delta R^{2}\right)$ & $+0.07 * * *$ & $+0.02 * * *$ & $+0.11^{* * *}$ & $+0.10^{* * *}$ \\
\hline Mealworm product $\left(\Delta R^{2}\right)$ & $+0.03 * *$ & $+0.01 * * *$ & $+0.22 * * *$ & $+0.17^{* * *}$ \\
\hline Total Model $\mathbf{R}^{2}$ & $0.37 * * *$ & $0.36 * * *$ & $0.46 * * *$ & $0.39 * * *$ \\
\hline
\end{tabular}

Hierarchical linear regressions performed adding first product type factors, then food appropriateness of mealworms, and then expected or experienced sensory-liking of the mealworm products. Standardized coefficients reported for full model, and changes in model $\mathrm{R}^{2}$ for each model are reported. Total model $\mathrm{R}^{2}$ is highlighted in bold. Significance levels indicated with $* p<0.05, * * p<0.01$, and $* * * p<0.001$.

Product type factors (i.e. product appropriateness, familiarity and sensory-liking) although significantly correlated with one another, had different effects on the willingness to buy mealworm products. Product type had a stronger influence on willingness to buy before tasting than after tasting. Familiarity with the product type was always a significant predictor of willingness to buy, where higher familiarity predicted a higher willingness to buy once and regularly. Sensory-liking of the product type was only significant as a predictor before products were tasted, where a more liked product type predicted a higher initial willingness to buy. Product appropriateness played a significant role only when regular consumption was considered, where higher product appropriateness (as evaluated before tasting) predicted a higher willingness to buy mealworm products for regular consumption. These results indicate that a familiar and liked product preparation may improve trial intentions, but product appropriateness is important when regular consumption is considered.

Expected sensory-liking was strongly correlated with the product type factors [appropriateness: Pearson's $r=+.59, p<.001$; familiarity: Pearson's $r=+.63, p<.001$; sensory-liking: Pearson's $r=+.44$, $p<.001]$ and when added to the model, only contributed to a slight increase in explained variance. After tasting, the experienced sensory-liking became an important predictor of willingness to buy mealworm products once and regularly, where a more positive taste experience predicted a higher willingness to buy. Food appropriateness of mealworms was always a significant predictor of willingness to buy. Higher food appropriateness predicted higher willingness to buy, but its predictive effect was stronger after products were tasted. These results indicate that after products are tasted, the quality of the sensory experience and food appropriateness of 
mealworms become more important in considering future willingness to buy. Overall, less than half of the total variance was explained by these factors, which suggests the importance of other factors in predicting purchase intentions. These factors are explored in the next section (6.3.3.3).

\subsubsection{Qualitative analysis of interest and willingness to buy regularly}

Open-ended responses regarding interest in trying and willingness to buy mealworm products regularly were content-analysed and summarized in Table 6.4 to further explore reasons underlying the evaluations.

Table 6.4. Frequency of codes for interest in trial and regular consumption of insect-based foods.

\begin{tabular}{|c|c|c|c|c|c|c|}
\hline \multirow[t]{2}{*}{ Categories } & & \multirow[t]{2}{*}{ Example Codes } & \multicolumn{2}{|c|}{ Unwilling tasters ( $n=79$ ) } & \multicolumn{2}{|c|}{$\begin{array}{c}\text { Willing tasters } \\
(n=135)\end{array}$} \\
\hline & & & Trial & Regular & Trial & Regular \\
\hline & + & tasty & 0 & 0 & 7 & 1 \\
\hline \multirow[t]{4}{*}{ Sensory } & - & not tasty, unknown taste & 5 & 13 & 6 & 32 \\
\hline & $+/-$ & tastiness & 0 & 1 & 19 & 12 \\
\hline & & Sub-total (\% total): & $5(5 \%)$ & $14(13 \%)$ & $32(13 \%)$ & $45(19 \%)$ \\
\hline & + & sustainable, protein, healthy & 4 & 2 & 104 & 2 \\
\hline \multirow[t]{4}{*}{ Product } & - & idea, gross, scary, not food, ugly & 79 & 71 & 17 & 47 \\
\hline & $+/-$ & healthiness, visibility, preparation & 3 & 6 & 13 & 21 \\
\hline & & Sub-total (\% total): & $86(84 \%)$ & 79(75\%) & $134(53 \%)$ & $70(30 \%)$ \\
\hline & + & openness, fun, curiosity & 5 & 2 & 52 & 5 \\
\hline \multirow[t]{4}{*}{ Individual } & - & not interested & 2 & 1 & 0 & 0 \\
\hline & $+/-$ & n.a. & n.a. & n.a. & n.a. & n.a. \\
\hline & & Sub-total (\% total): & $7(7 \%)$ & $3(3 \%)$ & $52(20 \%)$ & $5(2 \%)$ \\
\hline & + & cheap, food security & 0 & 0 & 11 & 0 \\
\hline \multirow[t]{7}{*}{ Contextual } & - & costly, social, how to prepare? & 4 & 8 & 8 & 95 \\
\hline & $+/-$ & cost, necessity, availability & 0 & 2 & 18 & 18 \\
\hline & & Sub-total (\% total): & $4(4 \%)$ & 10(9\%) & $37(14 \%)$ & $113(49 \%)$ \\
\hline & & \# positive codes (\% total): & $9(9 \%)$ & $4(4 \%)$ & $174(68 \%)$ & $8(3 \%)$ \\
\hline & & \# negative codes (\% total): & $90(88 \%)$ & $93(88 \%)$ & $31(12 \%)$ & $174(75 \%)$ \\
\hline & & \# neutral codes ( $\%$ total): & $3(3 \%)$ & $9(8 \%)$ & $50(20 \%)$ & $52(22 \%)$ \\
\hline & & Total \# of codes: & 102 & 106 & 255 & 233 \\
\hline
\end{tabular}

Codes were classified in 4 categories (sensory, product, individual, and contextual), and with 3 valences (+positive, -negative, +/-neutral) and frequencies were tabulated. Most frequently mentioned reasons for each category are highlighted in bold.

Comments from the unwilling tasters $(U)$ were mostly negative, with the most frequently cited reason being the 'idea' of eating insects (interest: 45x, eat regularly: 34x). Reasons mainly pertained to its appropriateness as food:

"It does not appeal to me to eat pests. I would not let any bite [of it] down my throat." 
In contrast, willing tasters (W) were very positive regarding their interest in insects as food, with many citing reasons of 'curiosity' (32x) and 'sustainable' (28x). The environmental and nutritional benefits of insects were often mentioned in relation to personal food goals and their curiosity regarding the sensory experience:

"I find it important that the protein I eat is sustainable and animal-friendly."

$[\mathrm{W}, 38 \mathrm{~F}]$

"Since mealworms are a more sustainable food source, I am very curious about the taste of it."

$[\mathrm{W}, 22 \mathrm{M}]$

Unlike unwilling tasters who mainly rejected the idea of insects as food, willing tasters mentioned many criteria that need to be satisfied in order for them to eat insects (e.g. tastiness, healthiness, product appeal, price, availability). For the willing tasters to eat insects regularly, factors relating to its usage were important, where they made the most mentions of 'how to prepare' (31x) and 'pricey' (27x). Many of these were negative and related to practical concerns (e.g. high price, low availability and lack of knowledge regarding its preparation) and social concerns such as difficulties when dining with others who do not accept insects as food. A few also mentioned about the taste experience being not bad but not sufficiently good to want to eat it again. Furthermore, willing tasters often mentioned the unfamiliar taste of insects, and considered insects to be scary and unattractive as food.

\subsection{DISCUSSION}

This study revealed how better products can improve the initial sensory-liking and willingness to buy a novel food, but only if consumers are motivated to eat it. Nevertheless, an unsatisfactory taste experience and low food appropriateness, together with other practical and socio-cultural concerns, could still prevent regular consumption. While research on the consumer acceptance of insects and other novel foods often focus on the psychological factors that determine the initial willingness to eat, here we explored how better products may improve the sensory appeal of insects as food and discuss the implications on encouraging trial and regular consumption of insect-based foods.

Meatballs that were higher in familiarity and sensory-liking than the dairy drink were perceived to be the more appropriate product type for incorporating mealworms. Correlations showed that familiarity and sensory-liking only partially related to product appropriateness, which may explain why adding familiar and liked ingredients do not always increase the willingness to eat a novel food (Stallberg-White \& Pliner, 1999). While meatball product type had an overall positive effect on the expected sensory-liking of the mealworm product, higher product appropriateness seemed to play an added role by lessening the negative impact of the mealworm's presence. 
This was also observed in a previous study (Chapter 5) where higher perceived appropriateness of the combination between mealworms and the product preparation resulted in a smaller 'contaminative effect' caused by adding mealworms to a familiar product. Predictions of willingness to buy further revealed that familiarity and sensory-liking of a product preparation could increase initial or trial intentions to buy, but product appropriateness is important when regular consumption is considered. In qualitative research involving Dutch consumers (Chapter 2), even though participants expressed a high willingness to try insect-based cookies due to the sensory-liking of the preparation, they did not perceive them to fulfil environmental goals and were not motivated to eat them regularly.

Higher expected sensory-liking of the mealworm products was strongly related to higher product appropriateness, but when products were tasted, the experienced sensory properties had a strong influence on sensory-liking. Descriptive and ideal sensory profiling revealed that mealworm products were expected and experienced to taste very different from the original mealworm-free products, but were generally preferred to taste similar to the original meatball or drink product. Hence, after having experienced the less appropriate mealworm drink to be more similar to the original drink, participants rated the experienced sensory-liking higher than expected, whereas the more appropriate mealworm meatball that was still experienced to be very different from the original meatball showed a drop in ratings of sensory-liking after tasting. Based on this outcome, it is likely that the mealworm products were judged according to the quality standards of the product category (i.e. meatballs, dairy drinks) (van Trijp \& van Kleef, 2008; Woodruff, Cadotte, \& Jenkins, 1983), which could pose a problem if the sensory properties of the insects do not fit well with the product type. However, even though consumers may expect a food substitute to taste similar to what it replaces (Hoek et al., 2011), a significant proportion of participants indicated unique mealworm attributes (e.g. nutty) when profiling their ideal mealworm products, which suggests that moderate levels of newness could also result in positive affect (Mandler, 1982). If so, it may not be necessary to entirely mask the properties of the insects when developing insect-based foods. Perhaps the right approach should be to find appropriate preparations that best fit the properties of the insects (Deroy et al., 2015; Shelomi, 2015), just like it is done in cultures where insects are eaten.

Willingness to buy mealworm products mostly decreased or remained the same after the taste experience, even though sensory-liking of the mealworm drink was significantly better than expected. Given the strong positive correlations between experienced sensory-liking and willingness to buy, our findings suggest that a more disappointing taste experience would lower intentions to buy, but the converse is not necessarily true. While taste strongly motivates food consumption, food choice decisions are also driven by motives beyond taste (Steptoe et al., 1995). According to Grunert and van Trijp (2014), a negative experience could quash further intentions of use, but a positive experience may not increase consumption intentions because 
extrinsic qualities (e.g. health, price) could also determine if there is sufficient incentive to adopt a new product. Furthermore, the perceived appropriateness of mealworms for consumption (i.e. food appropriateness) significantly predicted the willingness to buy, particularly after products were tasted. Chapter 3 showed that participants may be willing to try mealworm-based products despite their negativity, but food appropriateness strongly determined their willingness to eat again.

Willingness to buy products for trial consumption was always significantly higher than for regular consumption. Our qualitative findings demonstrated that the willing tasters expressed a high willingness to try mealworm products or insect-based foods largely due to their curiosity and interest in insects as a sustainable source of protein. While this was sufficient as an initial motivation, there were still many reservations regarding the regular consumption of insect-based foods, such as reasons of practical and socio-cultural concerns (e.g. high price, low availability, lack of preparation knowledge, poor acceptance amongst peers). Apart from that, some wrote that they experienced the mealworm products to be not tasty enough or too unfamiliar, and were slightly perturbed by the idea of eating insects. These findings were reflected in the qualitative research by House (2016), where initial motivations to eat insect-based foods differed greatly from repeat motivations, which were strongly based on other conventional considerations of food choice (e.g. taste, price, availability). Several others have also stressed the importance of considering these socio-cultural factors when attempting to introduce a culturally unfamiliar food like insects (Chapter 2; Lensvelt \& Steenbekkers, 2014; Looy et al., 2014; Shelomi, 2015).

This study revealed large individual differences in evaluations and motivations, where unwilling tasters perceived mealworms to be much less appropriate as food, and were neither willing to buy it nor to revise their intentions when a more appropriate product is presented. Unlike the willing tasters who represent a minority of early adopters (House, 2016; Verbeke, 2015), for the unwilling tasters, the product and sensory qualities seemed irrelevant as they did not want to eat insect-based foods, rejecting it on the basis that it is not food and is therefore disgusting to eat. Evidently, food products that contain disgusting ingredients could be rejected even without consideration of the ingredient's physical properties (Rozin \& Fallon, 1987). In addition, previous research identified willing consumers of insects to be neophilic young males (Hartmann et al., 2015; Verbeke, 2015). In this study, willing tasters were more neophilic young females. The young female cohort might be due to many Wageningen University students showing up for the sensory study, but individual differences in food neophobia suggest that certain people are more likely to taste insect-based foods, thereby changing their attitudes towards it.

\subsubsection{Implications of the research}

Outcomes of this study have several implications on product development and consumer acceptance. Due to the positioning of insects as a meat substitute (Shelomi, 2015; Tucker, 2014), 
there was a strong initial preference for savoury insect products. However, a good sensory experience still depended very much on how the combined product performs according to product category standards, and whether consumers will accept the unique properties of the insects within the food. Using familiar product preparations that are high in sensory-liking may lower the initial barriers of trial, but repeat consumption would require that the product is perceived to be appropriate in terms of the ingredient combination and context of consumption. It is also important to realize that making better products is only effective when people are at least, to some extent, motivated to eat the novel food. Even if initial motivations were strong, and product and sensory criteria satisfied, other contextual factors such as supply-side issues and socio-cultural aspects remain obstacles to regular consumption (House, 2016; Shelomi, 2015). Dietary practices are already difficult to change even when foods are familiar, due to the habitual and cultural nature of dietary patterns (Honkanen, Olsen, \& Verplanken, 2005; Rozin, 2006; Southgate, 1996). Substituting a highly valued food like meat for a novel and unappealing food like insects is, without a doubt, going to require even more extensive measures to encourage consumption. Efforts to introduce insects as food would certainly need to address the various individual, product and environmental barriers that are important in food behaviours (Meiselman, 2003; Shepherd, 2001).

\subsubsection{Limitations and future research}

In this study, we could not establish how specific sensory properties contributed to the experienced sensory-liking of the products. We expect that in the context of insect-based foods, the experienced sensory-liking of the products could also depend on the individual's expectations of how mealworm products should taste and its perceived appropriateness as food. Further work needs to be done to understand the sensory perceptions of highly novel foods and to examine how the relationships between product properties and sensory-liking could be established. This would contribute towards more actionable guidelines for developing insect-based foods. In addition to that, further investigations on the sensory performance of novel foods when processed and prepared differently could provide insights into the appropriate product types that would deliver the most satisfactory taste experience.

Another limitation of the study is that the willingness to buy the original products was not measured. While taste familiarity ratings provided a rough indication of whether the participant usually consumes the type of products (i.e. meatball or dairy drink), it is possible that a product could be familiar but is not usually bought at the store (e.g. homemade, consumed at parties). Investigating the effects of a single taste exposure also limits the conclusions that can be made regarding long term acceptance. Usually multiple exposures are required for food preferences to be developed (Birch, 1999). In this study, we examined the differences between willingness to buy for trial and regular consumption to engage participants into thinking about adoption of 
insect-based foods in the long run. Studies involving repeated and long term exposure would certainly be interesting to pursue, and could provide a better understanding of how consumer preferences towards culturally unusual foods like insects could develop over time. Finally, studies observing actual purchase and consumption behaviour would provide richer insights than selfreported behavioural measures when assessing the effectiveness of product strategies and identifying other barriers that may be currently unforeseeable.

\subsection{CONCLUSIONS}

This study used appropriate and inappropriate mealworm products to investigate product influences on the sensory-liking and willingness to buy insect-based foods for trial and regular consumption. Outcomes demonstrated that making better products contributes to the acceptance of a novel food by significantly improving the initial appeal and quality of the sensory experience, and help to lower the product-related barriers to consumption. However, consumers should already be motivated to eat insects, otherwise the product type has little influence on consumption intentions. While familiarity and sensory-liking of the product preparation helps to encourage trial, the combined product should also be appropriate and taste good if it is to be regularly consumed. Even with high interest and good products, motivated consumers still hesitate to consume insect-based foods regularly due to other practical and socio-cultural barriers. Based on the study findings, we recommend that future research should not only focus on increasing initial motivations towards eating insects, but should also explore the barriers to buying and preparing insects for regular consumption, where issues relating to availability, pricing, knowledge and the social environment could inhibit the uptake of this culturally new food.

\section{ACKNOWLEDGMENTS}

We are grateful to Eva van den Berg for her assistance in the product trials and preparing the samples for this study. We thank Arnout Fischer for his advice on the experimental design. 
CHAPTER 7 general DISCUSSION 


\subsection{INTRODUCTION}

Efforts in the West to introduce insects as sustainable alternative to meat face numerous challenges, particularly in terms of consumer acceptance of this culturally inappropriate food. While an increasing amount of attention is being devoted to understanding the consumer acceptance of insects, the focus has been very much on the psychological factors, leaving other important determinants of acceptance relatively unexplored. This thesis considers the cultural, taste and product aspects for a better understanding of the determinants of consumer acceptance of culturally inappropriate foods. Using cross-cultural focus groups (Chapter 2), hedonic and descriptive sensory studies (Chapters $3 \& 4$ ) and systematically-varied products (Chapters $5 \& 6$ ), this thesis provided insights into the various challenges faced when introducing a culturally inappropriate food to consumers. The key findings of the 5 empirical chapters are summarized in Table 7.1, and the implications on the consumer acceptance of insects as food in Western cultures and recommendations for future research and product development will be discussed in this chapter.

\subsection{OVERVIEW OF MAIN FINDINGS}

\subsubsection{Culture as a determinant of acceptance}

To provide insights into how culture influences the acceptance and rejection of insects as food, the cross-cultural focus group study described in chapter 2 used stage-wise discussions to examine the consumer perceptions and rationales in two cultural settings that differed greatly in exposure to insects as food. This chapter revealed that the main difference between those who accept or reject insects as food is largely explained in terms of their individual experiences. Those who had extensive experience with preparing and eating insects regarded insects to be very tasty, whereas Thai who lacked experience with eating insects ('non-eaters') rejected them as food for similar reasons as the Dutch participants ('eaters' and 'non-eaters'), i.e. that insects are strange and unattractive as food. In places where insects are eaten, culture provided the necessary setting within which locals can gain extensive knowledge and experience with obtaining, preparing and consuming insects, thereby learning to deeply appreciate insects as a food source. In the absence of cultural exposure to a food (i.e. Dutch context), other important considerations in food choice (e.g. taste, price, availability, knowledge of use) remain unsatisfied. Additionally, the availability of certain species and their typical local preparations within the cultural context (e.g. giant water bug chilli sauce, ant larvae omelette) contributed to culture-specific preferences for the more familiar species and preparations. 


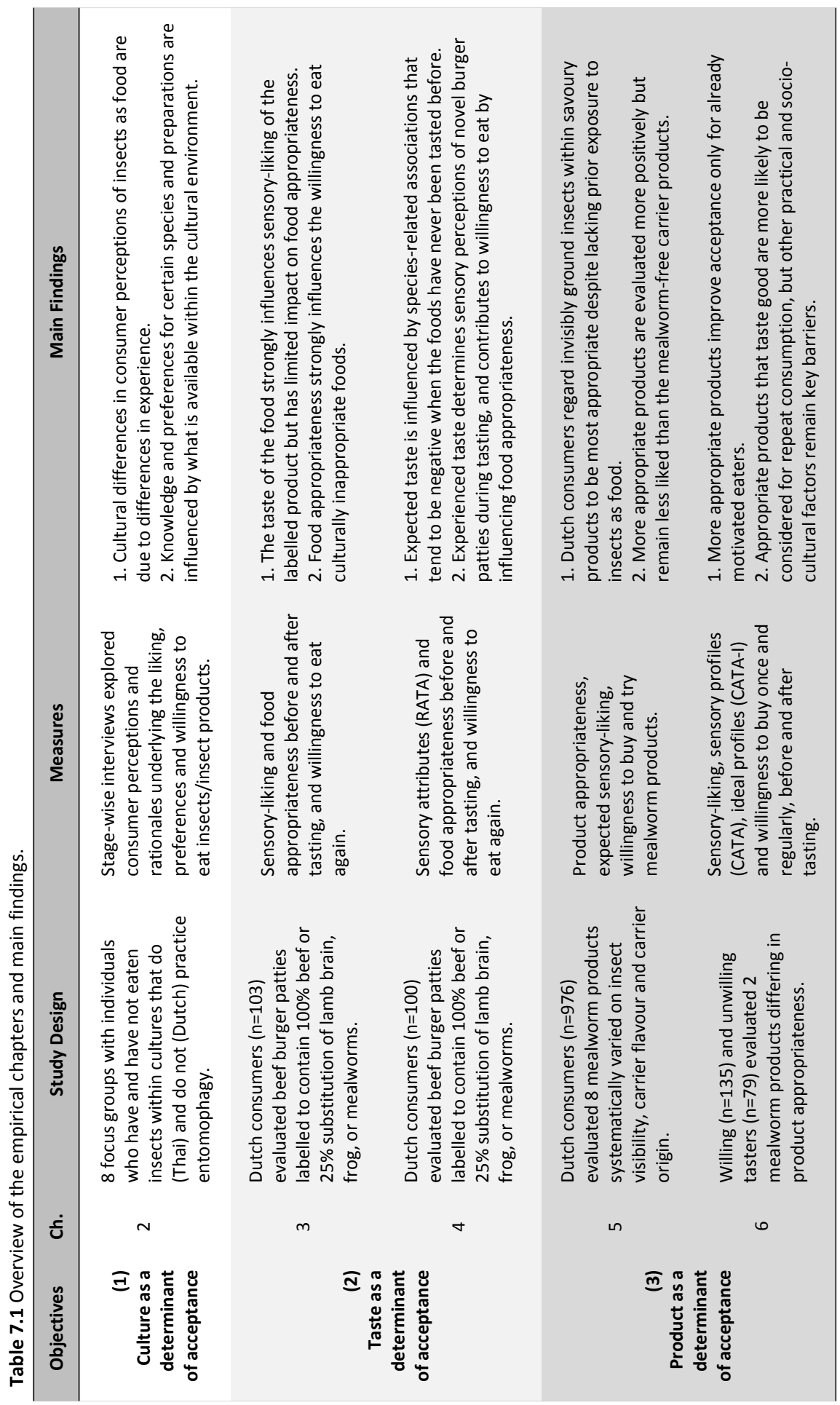




\subsubsection{Taste as a determinant of acceptance}

The role of taste as a determinant of acceptance was explored with the taste experiments described in chapter 3 and chapter 4, to gain insights into how the taste and identity of the food influences consumer perceptions and willingness to eat various culturally unusual foods (i.e. lamb brain, frog, mealworms). In these studies, beef burger patty recipe formulations were randomly presented with labels claiming $100 \%$ beef or $25 \%$ substitution with these novel ingredients. Chapter 3 revealed that the low willingness to eat such foods is heavily influenced by the perceived food appropriateness, while the actual taste of the food appeared to have a relatively minor influence on willingness to eat again. Chapter 4 explored the sensory perceptions and showed that consumers inferred the prospective taste of the novel foods based on speciesrelated associations (e.g. 'slimy' frog, 'mealy' mealworm) that tended to be negative when prior experience is absent. Outcomes of these studies suggest that while the taste of the food did not predict the willingness to eat again, the food's taste indirectly contributes to acceptance by influencing the perceived food appropriateness.

\subsubsection{Product as a determinant of acceptance}

In chapter 5 and chapter 6 , mealworm products were systematically varied to gain insights into how the product contributes to hedonic evaluations and consumption intentions of a culturally inappropriate food species. Similar to observations of Stallberg-White and Pliner (1999), findings showed that the effectiveness of hiding insects within familiar products is dependent on the perceived product appropriateness. Chapter 5 showed that Dutch consumers generally preferred savoury products because of the positioning of insects as a meat alternative, but even the most appropriate mealworm products are inferior to the original mealworm-free version. Chapter 6 showed that an appropriate product and its taste quality are particularly important when considering repeat consumption. However, appropriate and tasty products are likely to have little impact on the consumption intentions of consumers who are not already willing to eat insects. These chapters conclude that the acceptance of a culturally inappropriate food is not simply achieved by hiding it within familiar products. Disguising the food may lower initial barriers of trying a novel food, but the presence of the novel food can alternatively be seen to make the product less tasty and less appropriate for consumption. Other practical and socio-cultural barriers (e.g. price, knowledge, availability) are also major barriers to regular consumption. 


\subsection{THEORETICAL CONTRIBUTIONS:}

\section{CONSUMER ACCEPTANCE OF CULTURALLY INAPPROPRIATE FOODS}

This thesis examined the factors that influence the acceptance and rejection of insects as food in order to gain a better understanding of the determinants of consumer acceptance of culturally inappropriate foods. It extends the current body of literature that mostly focuses on the psychological determinants (e.g. food neophobia, motivations, risk perceptions) by giving more attention to the roles of culture, taste and the product. Through cross-cultural and sensory studies with systematically-varied products, more attention was given to the contextual factors that are critical to food choice and acceptance (Meiselman, 2003; Shepherd, 2001; Sobal \& Bisogni, 2009). Key contributions of the work of this thesis will be discussed below.

\subsubsection{Rejection of culturally inappropriate foods}

The rejection of culturally inappropriate foods is often investigated in relation to the cultural meanings given to the food (Fessler \& Navarrete, 2003; Meyer-Rochow, 2009). In the case of insects, the low consumer acceptance is linked to the Western image of insects and how insects do not fit Western ideas of food (Looy et al., 2014; Shelomi, 2015; Yen, 2009). The negative attitudes towards eating insects is thus usually blamed on Western culture and values (van Huis et al., 2013; Yen, 2009). By comparing across cultures that do and do not practice entomophagy, this thesis revealed that the abhorrence of insects as food is not an inherent part of 'Western culture' per se, but results from the fact that most Westerners lack direct experiences with insects as food. People from cultures (e.g. Thai) where insects are eaten also reject eating insects if they have not been earlier exposed to it (Chapter 2). The role of exposure is best reflected by Harris (1985) who suggests that "[t]he reason we don't eat [insects] is not that they are dirty and loathsome; rather, they are dirty and loathsome because we don't eat them."

Findings of this thesis indicated that certain potential food substances are rejected because unfamiliarity with their taste leads to other associations being triggered, and certain unfamiliar species may trigger more inappropriate associations than others (Chapters 2 and 4). Even though food rejections might be related to the animal status and aversive textural properties (Martins \& Pliner, 2006), this thesis suggests that these are merely learned associations gained over the course of life that are used to justify the rejections when the items have never been used as food (Chapters 2 and 4). Most Western consumers, lacking the experience with insects as food, have learned of insects in the non-food context, which are mostly negative and pertain to disease and decay. Such negative insect associations are not unique to Western culture, where Thai insect eaters also named such associations as reasons not to eat certain species that they were not familiar with (Chapter 2). A striking example was that of mealworms, which evoked strong associations with maggots that are often seen in decomposing carcasses in the Thai context. As 
such, mealworms were strongly rejected by the Thai eaters who would delightfully eat a giant water bug, despite its cockroach-like appearance that many Dutch find repulsive.

These insights bring light to why some of the more unusual local delicacies tend to be strongly rejected in other cultures. Various literature (Hopkins, 2014; Setchfield, 2010) have compiled lists of 'strange' foods from the Western perspective, which mostly include uncommonly eaten animal species such as dogs, cats, insects, bats, frogs and lizards, and non-muscle parts of conventional livestock animals such as tongue, genitals, head, blood, organs and feet. Animals eaten alive (e.g. octopus in Korea), raw (e.g. fish in Japan) or half-developed (e.g. duck embryo in Philippines) are also considered to be 'strange'. The fact that a large percentage of such foods tends to be of animal origin, has led to a strong belief that the animal's nature is responsible for food rejections (Fessler \& Navarrete, 2003; Pliner \& Pelchat, 1991). Yet this theory does not explain why certain plant-based foods such as flowers, durian, and various fermented foods are strongly rejected by those who are unaccustomed to eating them. Instead, findings of this thesis suggest that the main factor that separates eaters and non-eaters of various foods lies in whether the prospect of consumption evokes memories of past taste experiences or associations that are inappropriate in the food context. Hence, common meanings that people attach to certain animals (e.g. pets, pests, spiritual) (Fieldhouse, 1996) may be more likely to contribute to greater feelings of inappropriateness and lead to stronger rejections.

\subsubsection{Food neophobia and other individual traits}

Food neophobia, or the tendency to avoid novel foods, is regarded to be a major predictor of the acceptance of novel foods. Studies consistently show that more neophobic persons are less willing to eat novel foods than neophilic persons and also express a greater tendency to dislike the foods (Fenko et al., 2015; Raudenbush \& Frank, 1999; Tuorila et al., 2001). This relation was observed across the studies of this thesis (Chapters 3-6), but outcomes indicated that food neophobia only has minor influence on consumers' willingness to eat culturally inappropriate foods. Studies in this thesis (Chapters 3 and 5) also showed that evaluations varied significantly with socio-demographic variables where younger males were generally more willing to try (Ruby et al., 2015; Verbeke, 2015), but that did not necessarily mean that they liked the products better, or were more willing to buy them. These findings contrast with those of previous studies on Western acceptance of insects as food, where food neophobia usually emerges as a strong predictor of willingness to eat, along with other individual factors like age and gender (Hartmann et al., 2015; Verbeke, 2015).

Despite significant individual differences in willingness to eat insects due to food neophobia and socio-demographic variables, they do not account for the low acceptance of culturally inappropriate foods like insects. A Flemish study about sustainable meat alternatives showed that the most adventurous and ethically-motivated consumers could be much more willing 
to eat various meat substitutes, but had no intention to eat foods containing insect proteins (Vanhonacker et al., 2013). Verbeke (2015) also reports less than 3\% of Belgian consumers being definitely willing to adopt insects as a meat substitute. These observations suggest that even though certain individuals may be more interested and positive about novel foods, radically new and culturally inappropriate foods could trigger strong negative reactions (e.g. disgust) that may override a person's interest in eating (van Trijp \& van Kleef, 2008).

In addition, chapter 2 showed that being neophilic is not a necessary condition for accepting insects as food. Thai eaters of insects were not particularly neophilic and expressed a strong preference for familiar local foods, but had learned to accept insects as food through exposure since childhood. In the Dutch context where insects are novel food, certain individuals (i.e. neophilic, younger, male) were indeed more likely to have tried insects before and more willing to try products in spite of negative taste expectations (Chapters 3 and 5). This implies that there is higher chance that these individuals, who are more inclined to try novel foods, will be more likely to directly experience the food and alter their perceptions of it. Nevertheless, amongst the Dutch participants, some neophobic individuals reported to have tasted insects before (Chapter 5) and chose on their own will to taste the unusual novel foods during the sensory sessions (Chapters 3 and 4). This indicates that under certain circumstances, people who normally would prefer not to consume novel foods could possibly be influenced to taste it. For instance, chapter 3 showed that willingness to eat is higher in special occasions than in a regular meal setting, and chapter 2 showed that peer influence played a role during focus group interactions where several reluctant persons were convinced by other participants to try products. These findings suggest that future research should focus less on individual differences and more on identifying effective strategies to encourage consumption.

\subsubsection{Tasty but nasty}

The lack of sensory appeal is said to be a major barrier to acceptance (Tucker, 2014) and improvements to taste are likely to be more effective than rational arguments when introducing culturally new foods like insects (Deroy et al., 2015). By separating the effects of the food's identity (i.e. label effects) and the experienced sensory properties (i.e. recipe effects), this thesis showed that the low sensory appeal had little to do with the actual food properties (Chapters 3 and 4). Prior to tasting, consumers had little knowledge regarding the food's taste, relying instead on species-related associations (e.g. 'mealy' mealworms) to construct taste expectations (Chapter 4). After tasting, even though participants acknowledged the good taste of the products, willingness to eat the foods again remained low due to the low food appropriateness (Chapters 3 and 4). These findings confirm earlier research that the experienced sensory properties are the basis for evaluating sensory-liking during consumption of a food (Cardello \& Maller, 1982; Raudenbush \& Frank, 1999). It additionally highlights the need to distinguish between liking a food's taste (i.e. 
sensory-liking), and liking to eat the food (Berridge, 1996), which is usually represented by more general terms of 'liking', 'palatability', and 'acceptance' in food literature. This is particularly important when considering culturally inappropriate foods, where the dislike of the food could be due more to the dislike of eating such foods than to the actual taste qualities of the food. Nevertheless, food appropriateness and sensory-liking are strongly correlated (Chapters 3 and 4), where good taste is still necessary to improve food appropriateness. It is, however, insufficient to encourage repeat consumption, which calls for a need to implement other strategies and incentives (Wansink, 2002).

New questions were raised regarding what is considered to be tasty when taste experiece is lacking, as food preferences are developed through experience (Birch, 1999). Consumers usually evaluate the product experience against their preferences (Cardello, 1995) or expectation standards that are developed through exposure to other products within the same or related category (Cadotte, Woodruff, \& Jenkins, 1987; Woodruff et al., 1983). Studies of novel food acceptance generally consider how the familiar flavours and product preparations improve the willingness to eat a novel food (Prescott et al., 2004; Stallberg-White \& Pliner, 1999; Tuorila, Andersson, et al., 1998). Hence, hiding the novel foods within familiar products is deemed to improve acceptance by maintaining a similar appearance to familiar foods (Gmuer et al., 2016; Shelomi, 2015; Wansink, 2002). Outcomes of this thesis suggest that this may not be the correct frame of reference, as the presence of the novel food may instead be perceived as an inferior version of the original product. Hedonic, descriptive and ideal profiling of original and novel products revealed two challenging aspects (Chapters 3-6). First, consumers expected that the novel product ideally possesses taste qualities similar to the original product, while the novel ingredient inevitably changes the properties of the product (Chapters 4 and 6). Second, the mere knowledge that a culturally inappropriate food is present is sufficient to make the product less appealing to eat (Chapters 3 and 5). While more psychological experiments are needed in order to establish consumers' frame of reference, these preliminary findings suggest that the consumer acceptance of products made from culturally inappropriate foods might benefit from considering its value in relation to existing products on the market.

\subsubsection{Making appropriate products}

The starting point for consumer-oriented product development lies in meeting consumer expectations (van Trijp \& Steenkamp, 2005). Yet consumer expectations regarding how certain foods should be appropriately prepared are formed through cultural exposure and past experiences of what tastes good together (Giacalone \& Jaeger, 2016; Rozin, 2006). For culturally unfamiliar foods, expectations are less defined (Pliner \& Stallberg-White, 2000; Stallberg-White \& Pliner, 1999). In the case of insects, its primary positioning as a sustainable alternative to meat has created expectations that insects be most appropriately used as a meat substitute 
(Chapters 2, 5, 6). This gives rise to two sets of dilemmas regarding how appropriate products from culturally inappropriate foods can be developed. The first pertains to its visibility within the product, where seeing is daunting but hiding does not guarantee that consumers consider the product to be tasty and appropriate for consumption. Deroy et al. (2015) and House (2016) also expressed reservations regarding whether consumers see the value that insects bring to the food product if it is not perceivable within the food. The second dilemma pertains to the carrier product, where a better liked product type generally helps to improve the expected sensoryliking and consumption intentions of a culturally inappropriate food, but could result in greater feelings of contamination. For instance, a brownie may make a mealworm taste better, but the mealworms could make the brownie much less appealing to consume. Making appropriate products from culturally inappropriate foods is certainly not as straightforward as hiding it within familiar and liked products, although it may help to facilitate the process of acceptance by increasing the likelihood of trial. Ultimately more work needs to be done in creating products that are not only visually appealing, but also tastes good in relation to comparable products.

\subsubsection{Trying but not eating}

Initiating food trial is a necessary first step to learning to accept its qualities. Research into the consumer acceptance of novel foods thus often focuses on understanding the factors that contribute to the initial willingness to try (Martins \& Pliner, 2005; Ruby et al., 2015; Tuorila et al., 2001; Verneau et al., 2016). Results of this thesis showed that this provides a very limited view of consumer acceptance. The motivations underlying initial trial are different from that of repeat consumption. Making this distinction is therefore especially important when investigating the consumer acceptance of novel foods. This is because people tend to show both interest and reluctance in trying new foods (Martins \& Pliner, 2005; Rozin \& Vollmecke, 1986) but lack the knowledge regarding the product experience (Alba \& Hutchinson, 1987; Dick et al., 1990). Decisions to try a food could therefore be independent of the actual properties of the food and how much the taste is liked by the consumer (Chapters 2 and 3). Within this thesis, consumers were generally more willing to try the novel products than to buy it in the supermarket (Chapter 5). They were also more willing to buy it once for trial than to buy it for repeated consumption (Chapter 6), and were willing to try products under certain circumstances (e.g. experiments, events, special occasions) even if they do not expect them to taste good or consider it inappropriate for consumption (Chapters 2-4). These outcomes indicate that studies that investigate the acceptance of novel foods in terms of initial willingness to try are likely to give a short-term and overly optimistic view of the likelihood that consumer acceptance can be achieved. Once products are tasted, the quality of the experience would play an important role in determining repeat consumption (Steenkamp \& van Trijp, 1996). 
Culturally inappropriate foods face additional challenges that other novel foods do not. This thesis revealed that when considering repeat consumption, consumers are not only concerned about the taste of the food (Chapter 6), they are also influenced by the food's appropriateness for consumption (Chapter 3), and numerous other practical (e.g. cost, convenience) and socio-cultural (e.g. availability, knowledge, social influence) factors (Chapters 2 and 6). Food behaviors are not shaped in a vacuum, and people are very much influenced by the environment (Meiselman, 2003; Shepherd, 2001; Sobal \& Bisogni, 2009). By comparing the Thai and Dutch contexts, it became clear that culture provides the environment within which choices are made and habits are established (Fieldhouse, 1996; Rozin, 1996; Southgate, 1996). It follows that the cultural environment constrains attempts to make significant dietary changes towards foods that are unfamiliar and less accessible. Hence, even the most motivated and willing Dutch consumers (Chapters 2 and 6) expressed that their lack of knowledge regarding the distinctive tastes and methods of preparing insects, as well as the low availability, high price and lack of acceptance amongst dining companions (i.e. family, friends) are major reasons for not wanting to regularly consume insect-based foods. The importance of these socio-cultural factors has been further examined in the work of House (2016) and Shelomi (2015), both of whom address the need to provide a more conducive environment if real consumption of insects in the West is to be achieved.

\subsection{PRACTICAL IMPLICATIONS: INTRODUCING INSECTS AS FOOD IN THE WEST}

The introduction of insects as food in the West primarily aims at reducing the environmental impact of meat by promoting the consumption of more sustainable alternatives (van Huis et al., 2013; Yen, 2009). Yet the low consumer acceptance is a major obstacle that has received attention from researchers only very recently. This thesis delved into the role of culture, taste and the product as determinants of the initial and repeat consumption of insect-based foods, revealing insights into the numerous challenges and dilemmas encountered when introducing a culturally inappropriate food like insects to Western consumers.

The question of whether insects will ever gain acceptance as food in the West rests on the minds of many. This is because the environmental benefits of insect consumption will not be realized unless consumers are willing to consume it to some extent and as a substitute for meat products. Current rational approaches may serve to create greater interest and openness to considering insects as food but are unfortunately insufficient to establish regular consumption of this new food (Looy \& Wood, 2006). Promoting foods on the basis of functionality (Frewer, Scholderer, \& Lambert, 2003; Siro et al., 2008; Urala \& Lähteenmäki, 2004; Verbeke, 2005) or sustainability (Tobler et al., 2011; Vanhonacker et al., 2013; Vermeir \& Verbeke, 2006) is already challenging for 
regular foods, not to mention foods that are culturally inappropriate. Furthermore, the current positioning of insects as a meat alternative has added challenges for consumer acceptance. Meat is highly valued by Western consumers and is strongly embedded within the Western diet (Grunert, 2006; Hoek et al., 2011; Schösler, de Boer, \& Boersema, 2012), making it particularly difficult to replace.

Admittedly, this thesis provides a rather bleak outlook regarding the potential for insects to become food in the West. While it does not claim it to be impossible, it gives a much less optimistic view than shown by Caparros Megido et al. (2014) after taking into account the various dimensions of food acceptance. This thesis emphasizes that consumer acceptance entails much more than the initial willingness to eat a food, because the high interest in this novel and sustainable food could mean that many are willing to try out of curiosity without having any intentions of eating it again. To sum up the outcomes of this thesis, the low perceived food appropriateness, negative taste expectations, unsatisfactory taste experiences, lack of knowledge regarding the use of insects as food, and a cultural environment that is not conducive for insect consumption (e.g. high price, limited availability of good products, rejection by peers) all make it difficult for even the most open-minded and motivated consumer to accept insects as a regular food. Shelomi (2015) argues that supply-side issues still need to be addressed and insects should also provide relative advantages over existing foods on the market, to provide incentives for Western consumers to choose insects over foods that are currently available.

Unlike in cultures where people have accumulated rich experiences and knowledge regarding the procurement, preparation and consumption of locally-available species and products (Hanboonsong et al., 2013; Obopile \& Seeletso, 2013; Yhoung-Aree et al., 1997), Western consumers not only have to learn to use and appreciate insects as food, they also have to unlearn their prejudices against insects (Looy et al., 2014). This thesis established that riding on people's curiosity may help to initiate trial and improve people's opinions towards its taste, but the perceived inappropriateness of insects as food is much more deeply ingrained and resistant to change (Chapters 3 and 4). A key challenge is therefore to ensure the continued consumption after the first curious experience, where future research should focus less on the initial willingness to try and more on the strategies that will help to sustain consumption and make real changes in attitudes towards eating insects.

This brings us to the next question of how can insects be introduced as food in the West? Unlike familiar foods where consumers have established preferences towards certain product qualities, this thesis showed that Western consumer wishes regarding eating insects was related more to their desire not to eat it and the conditions that need to be fulfilled in order to enable them to eat it (e.g. not seen, not tasted, cheap, convenient) (Chapters 2 and 6). Therefore current efforts to introduce insects as a source of protein focus mainly on ways that insect proteins can be unperceivably incorporated in familiar products, either by grinding small amounts of 
insects to be added to vegetarian-type products or by extracting the insect proteins such that they can be used in various food applications (van Huis, 2013; Yi et al., 2013). This approach is in contrast to cultures where insects are eaten, where the distinctive tastes and textures of the various species are appreciated by consumers and used to enhance the sensory value of the dish or meal. Following the arguments of Deroy et al. (2015) and House (2016), there is a need to consider whether hiding insects within existing food products is the way to go as consumers may not perceive an added value of incorporating insects within the food. Furthermore, technological advancements to mask the properties of the insects would be needed.

Even though it is not clear yet in what form insects should enter the Western market, insights from this thesis stressed the importance of considering the product dimensions when researching and developing insect-based foods for Western consumers (Chapters 2, 5 and 6). Unlike the Thai insect eaters who recall tasty memories of eating insects when asked to eat insects, consumers who have little or no experience with insects as food tend to harp on the typical insect image as pest and disease-carriers (Harris, 1985; Looy et al., 2014). Presenting insect-based products to Dutch consumers helped to shift attention to the specific food qualities of the insects. The stagewise discussions in chapter $\mathbf{2}$ showed that for consumers who know little about the use of insects as food, presenting an appropriately prepared insect product certainly has more appeal than the general idea of eating creatures that are generally regarded as pests and filth. On the other hand, an inappropriate product (e.g. mealworm muffin) could potentially elicit more negative associations (e.g. rotting food) and result in stronger rejections of the food.

Creating appropriate products is not straightforward for this culturally unfamiliar and inappropriate food. The challenges that are likely to be faced in product development are summarized in Table 7.2. In the Western context, the primary positioning of insects as a sustainable meat alternative has created expectations that insects are most appropriately used as meat substitutes (Chapters 2, 5 and 6). Hiding insects within highly liked sweet products (e.g. brownies) may achieve trial but is unlikely to achieve acceptance as consumers do not perceive it to match the environmental goal and also do not consider meat to combine well with sweet foods.

Using insects as meat replacers poses further difficulties. Consumers then expect the insect product to imitate the taste and appearance of meat (Hoek et al., 2011), whereas the properties of most available insect species in Europe (i.e. mealworms, grasshoppers) differ greatly from conventional meat (i.e. beef, chicken). Mealworm meatballs, although considered an appropriate product, did not taste as good as an original meatball (Chapter 6). Shelomi (2015) suggests that the nutty flavour of certain insects may combine better with nut-based rather than meat-based products, but this may again stray from original motivations to use insects as a meat alternative. Not to mention, the cultural inappropriateness of insects as food means that the mere idea of its presence within the food is sufficient to result in dislike and rejection (Rozin \& Fallon, 1987). This implies that tasty products may be developed, but some consumers simply do not wish to 
Table 7.2. Challenges for product development

\begin{tabular}{ll}
\multicolumn{1}{c}{ Consumer wishes } & \multicolumn{1}{c}{ Challenges for product development } \\
\hline $\begin{array}{l}\text { - Insects are most appropriately used as a meat } \\
\text { alternative. }\end{array}$ & $\begin{array}{l}\text { - Insects have different properties from meat and might not } \\
\text { fit well with the preferred product preparation }\end{array}$ \\
$\begin{array}{l}\text { - Insects should be invisibly incorporated } \\
\text { within familiar and liked products }\end{array}$ & $\begin{array}{l}\text { - Insects may be perceived to contaminate or create a more } \\
\text { inferior version of the original product. }\end{array}$ \\
$\begin{array}{l}\text { - Insects should not make the taste of the } \\
\text { product worse. }\end{array}$ & $\begin{array}{l}\text { Technological challenges of masking the properties of the } \\
\text { insects or finding products with which its properties are } \\
\text { compatible. }\end{array}$ \\
$\begin{array}{l}\text { - Insects are not food and are therefore not } \\
\text { meant to be ingested. }\end{array}$ & $\begin{array}{l}\text { The knowledge of insects being present within the food is } \\
\text { sufficient to result in its rejection. }\end{array}$ \\
\hline
\end{tabular}

eat insects, in which case even the most appropriate and tasty products would not be effective in encouraging consumption (Chapter 6).

The lack of knowledge in the West regarding the use of insects as food presents further technological challenges. Unlike Thai eaters who know that roasting a cricket makes it crispy and easier to eat or that the strong fruity flavour of the giant water bug enhances the flavour of chilli sauces, such specific knowledge is lacking in cultures where insects are not eaten (Chapter 2). The development of attractive dishes requires a good understanding of how the flavours and textures of the ingredients match one another (Ottenbacher \& Harrington, 2008). At the outset of this thesis, it was observed that freeze-dried insect ingredients (e.g. mealworms) available in the Netherlands reeked of a rancid off-flavour that was detrimental to the taste of products they are incorporated in. This off-flavour is due to the high content of poly-unsaturated fatty acids (Ghaly \& Alkoaik, 2009; Siemianowska et al., 2013; Yi et al., 2013) that are highly prone to oxidation that results in these off-flavours (Gray, Gomaa, \& Buckley, 1996; Ladikos \& Lougovois, 1990; Malcolmson, Vaisey-Genser, Przybylski, \& Eskin, 1994). An exploratory study conducted during the course of this thesis (results not reported) investigated the effects of various processing methods (e.g. freeze-drying, blanching) and storage conditions (e.g. temperature) on the extent of lipid oxidation over time, and showed that storage of freeze-dried mealworm products at room temperatures (i.e. a form currently available in Dutch stores) contributes to particularly high levels of fat oxidation, that delivers a noticeable off-flavour to products they are added to. Even when mealworms are used fresh, they had a characteristic flavour and texture (e.g. nutty, crunchy) that influenced the taste of products when incorporated as an ingredient (Chapter 6). More work certainly needs to be done to explore the physicochemical and sensory properties of insect-based ingredients if products of good sensory quality are to be developed.

Based on the various findings, the way to go when introducing insects as food to Western consumers is likely to entail a mix of strategies. First, consumer perceptions need to be changed, 
where people have to learn to eat and appreciate the qualities of insects as food. In order to do so, more work has to be done on how to influence people's willingness to try insect-based foods, and to create products that taste good enough that people are not permanently aversive to eating it again. Second, incentives should be in place to encourage repeated consumption, especially since a single taste exposure is insufficient to develop taste preferences and to eradicate any feelings of inappropriateness. Third, communicating the taste of insect-based foods might help reduce uncertainty regarding its taste and reduce the negative expectations that are built from insect-related associations. This might reduce the disappointment upon realization that it does not taste anything like meat, and help consumers to appreciate its unique tastes. Fourth, the lack of knowledge regarding how to prepare insects for consumption should be aided with the availability of good recipes that complements the sensory qualities of the insects, rather than just using it as a protein supplement. Last but not least, more attractive prices and greater convenience in obtaining and using insect-based ingredients and products is needed to support the regular use of insects as food.

Finally, one needs to ask whether the environmental goal will be achieved through eating insects? The environmental advantages provided by insects are in relation to conventional meats (van Huis et al., 2013), and a vegetarian diet would still be more sustainable than one that includes insects (Shelomi, 2015). This thesis and several other studies (House, 2016; Verbeke, 2015) have highlighted that people who are most open to eating insects are those who are already motivated to eat more sustainably, and were willing to consider eating insect-based products as a means to expand their 'vegetarian' options. Meat lovers, on the other hand, may be curious to try insectbased foods but showed little interest in regularly consuming insect-based foods, especially since it does not taste as good (House, 2016). Ironically, introducing insects as food might result in a less sustainable outcome than if insects were not introduced at all. Furthermore, apart from the few insect-based meat substitutes (e.g. burgers, schnitzels) that are currently available in the Netherlands, the wide range of retail products that include cricket flour to cricket chocolates, cookies, energy bars and tortillas, as well as Western recipe books (e.g. Gordon (2013); van Huis et al. (2012)) seem to be guiding Western consumers towards eating more insects, but not necessarily in ways that serve the environmental goal. In light of these observations, perhaps we should not be asking whether insects will ever gain acceptance as food in the West, but whether achieving that will help to achieve the goal of improving food security? Efforts from policy makers, food providers and scientists should be aligned according to this goal if the advantages of eating insects are ever to be realized. 


\subsection{METHODOLOGICAL LIMITATIONS AND FUTURE RESEARCH}

This thesis covered a broad range of factors and combined approaches of consumer and sensory research to gain new insights into a topic that was relatively unexplored. In doing so, there were several methodological limitations to the conclusions of the research studies.

First, the use of self-reported measures has its limitations, as filling in questionnaires tends to result in certain rationalizations, especially when consumers are asked to report on products that are not usually thought about (Köster, 2009). Moreover, when evaluating radically new products, consumer responses are unstable and can easily be influenced by the way questions are framed (Hoeffler, 2003). Within this thesis, session observations and informal post-session discussions with the participants provided some insights into consumer behaviour that were not captured by the questionnaire. Observations during the focus group sessions (Chapter 2) revealed that social interactions and individual personalities played a very important role in people's decision to taste products. Some participants declared that they would never eat certain insects, but upon seeing that others around the table enthusiastically tasted them, giving positive reviews, and offering more information regarding the experience, several changed their minds and tasted the products. In the studies described in chapter 3 and chapter 4 , the experimental setting had a strong influence and almost all participants tasted the products despite low ratings of food appropriateness and willingness to eat ratings. Session observations revealed that many of those who tasted the products did so with great hesitation - first settling their nerves, then inspecting the food in small pieces, then only tasting a small quantity. While the self-reported ratings gave useful insights into the factors that influence acceptance, future research on novel food acceptance could greatly benefit from observational studies that could provide guidance when interpreting quantitative findings.

Second, this thesis mainly examined the effects of a single taste exposure on hedonic evaluations and consumption intentions. At the same time it was clear that mere taste exposure to an insect-based food is incomparable to the rich and varied experiences that Thai consumers have accumulated through different occasions over the course of a lifetime. Even in the case of innovations on regular foods (e.g. new drink flavour), multiple exposures are required to achieve significant improvements in liking and willingness to consume the products (Birch \& Marlin, 1982; Pliner et al., 1993; Tuorila \& Cardello, 2002). One could then expect that the deeply ingrained ideas relating to a culturally inappropriate food would take even more exposures over a course of time to have a real impact on attitudes and preferences. Future studies involving long term exposure could provide better insights into the potential for consumer acceptance (Chung \& Vickers, 2007; Hoek et al., 2013; Vickers \& Holton, 1998).

In this thesis, the product formulations may have limited the conclusions regarding how the liking of the taste experience potentially influences the uptake of culturally inappropriate foods. In the 
studies of chapter 3 and chapter 4, products claimed to contain lamb brain, frog and mealworms did not actually contain these ingredients. This was done in order to enable the randomization of recipe and labels to explore the influence of the food's taste and identity separately without causing participants to unknowingly ingest foods they would not wish to eat (tasting was optional). To make the burger patties taste different but still credible with each label, only slight modifications to a standard beef burger patty were made using plant-based ingredients, resulting in all the recipes tasting good. This limits the conclusions on (a) whether the actual properties of these culturally inappropriate foods will be accepted when experienced by Western consumers, and (b) whether recipes that ranged from extremely good to extremely bad in taste would result in the taste of the product playing a much more important role in determining the willingness to eat it again. Future studies that take into consideration the food's properties and the impact of good and bad taste experiences would provide useful insight into whether sensory-based approaches (e.g. Deroy et al. (2015)) might help to establish preferences for new foods.

\subsection{CONCLUSIONS}

The research described in this thesis brought new insights into the complexities involved in introducing a culturally inappropriate food by exploring the role of culture, taste and the product as determinants of acceptance. It demonstrates the importance of recognising the distinctiveness of various species and preparations when investigating the acceptance of a new class of food, where the general idea of eating a food species could evoke very different feelings and perceptions than when actually presented in a specific food context. Achieving consumer acceptance of a culturally inappropriate food is by no means a simple mission. There are numerous challenges and dilemmas in changing consumer perceptions and establishing a system surrounding the use of a new source of food. This thesis concludes that current efforts to address the consumer acceptance of insects as food in the West has only considered the tip of the iceberg, i.e. how to get people to try. Yet trying is not eating. Whether Western consumers will someday learn to appreciate the qualities of insects as food and eat it regularly will depend very much on appropriate strategies and incentives to help consumers overcome the negative perceptions and establish new eating habits within their cultural environment. 
REFERENCES 
Ahn, Y.-Y., Ahnert, S. E., Bagrow, J. P., \& Barabási, A.-L. (2011). Flavor network and the principles of food pairing. Scientific reports, 1.

Alba, J. W., \& Hutchinson, J. W. (1987). Dimensions of consumer expertise. Journal of Consumer Research, 411-454.

Aqueveque, C. (2015). Intention to try unfamiliar food: The role of level and type of unfamiliarity, food neophobia, and ethnocentrism. Journal of Food Products Marketing, 21(3), 244-254.

Ares, G., Bruzzone, F., Vidal, L., Cadena, R. S., Giménez, A., Pineau, B., Hunter, D. C., Paisley, A. G., \& Jaeger, S. R. (2014). Evaluation of a rating-based variant of check-all-that-apply questions: Rateall-that-apply (RATA). Food Quality and Preference, 36, 87-95.

Arvola, A., Lähteenmäki, L., \& Tuorila, H. (1999). Predicting the intent to purchase unfamiliar and familiar cheeses: the effects of attitudes, expected liking and food neophobia. Appetite, 32(1), 113-126.

\section{B}

Bäckström, A., Pirttilä-Backman, A. M., \& Tuorila, H. (2004). Willingness to try new foods as predicted by social representations and attitude and trait scales. Appetite, 43(1), 75-83.

Baker, M. A., Shin, J. T., \& Kim, Y. W. (2016). An exploration and investigation of edible insect consumption: The impacts of image and description on risk perceptions and purchase intent. Psychology \& Marketing, 33(2), 94-112.

Banović, M., Fontes, M. A., Barreira, M. M., \& Grunert, K. G. (2012). Impact of product familiarity on beef quality perception. Agribusiness, 28(2), 157-172.

Bar-Anan, Y., Liberman, N., \& Trope, Y. (2006). The association between psychological distance and construal level: Evidence from an implicit association test. Journal of Experimental PsychologyGeneral, 135(4), 609-622.

Barrios, E., \& Costell, E. (2004). Review: use of methods of research into consumers' opinions and attitudes in food research. Food Science and Technology International, 10(6), 359-371.

Beneke, J., Flynn, R., Greig, T., \& Mukaiwa, M. (2013). The influence of perceived product quality, relative price and risk on customer value and willingness to buy: a study of private label merchandise. Journal of Product \& Brand Management, 22(3), 218-228.

Berridge, K. C. (1996). Food reward: brain substrates of wanting and liking. Neuroscience \& Biobehavioral Reviews, 20(1), 1-25.

Birch, L. L. (1999). Development of food preferences. Annual Review of Nutrition, 19(1), 41-62.

Birch, L. L., \& Marlin, D. W. (1982). I don't like it; I never tried it: effects of exposure on two-year-old children's food preferences. Appetite, 3(4), 353-360.

Bodenheimer, F. S. (1951). Insects as human food: a chapter of the ecology of man: W. Junk The Hague.

Boland, M. J., Rae, A. N., Vereijken, J. M., Meuwissen, M. P., Fischer, A. R., van Boekel, M. A., Rutherfurd, S. M., Gruppen, H., Moughan, P. J., \& Hendriks, W. H. (2013). The future supply of animal-derived protein for human consumption. Trends in Food Science \& Technology, 29, 62-73.

Bredahl, L. (2004). Cue utilisation and quality perception with regard to branded beef. Food Quality and Preference, 15(1), 65-75.

Bruzzone, F., Ares, G., \& Gimenez, A. (2012). Consumers' texture perception of milk desserts. II Comparison with trained assessors' data. Journal of Texture Studies, 43(3), 214-226. 
Bukkens, S. G. F. (1997). The nutritional value of edible insects. Ecology of Food and Nutrition, 36(2-4), 287-319.

\section{C}

Cadotte, E. R., Woodruff, R. B., \& Jenkins, R. L. (1987). Expectations and norms in models of consumer satisfaction. Journal of Marketing Research, 305-314.

Calantone, R. J., Chan, K., \& Cui, A. S. (2006). Decomposing product innovativeness and its effects on new product success. Journal of Product Innovation Management, 23(5), 408-421.

Caparros Megido, R., Gierts, C., Blecker, C., Brostaux, Y., Haubruge, É., Alabi, T., \& Francis, F. (2016). Consumer acceptance of insect-based alternative meat products in Western countries. Food Quality and Preference, 52, 237-243.

Caparros Megido, R., Sablon, L., Geuens, M., Brostaux, Y., Alabi, T., Blecker, C., Drugmand, D., Haubruge, É., \& Francis, F. (2014). Edible Insects Acceptance by Belgian Consumers: Promising Attitude for Entomophagy Development. Journal of Sensory Studies, 29(1), 14-20.

Cardello, A. V. (1995). Food quality - relativity, context and consumer expectations. Food Quality and Preference, 6(3), 163-170.

Cardello, A. V., \& Maller, O. (1982). Relationships between food preferences and food acceptance ratings. Journal of Food Science, 47(5), 1553-1557.

Cardello, A. V., Schutz, H., Snow, C., \& Lesher, L. (2000). Predictors of food acceptance, consumption and satisfaction in specific eating situations. Food Quality and Preference, 11(3), 201-216.

Cardello, A. V., \& Schutz, H. G. (1996). Food appropriateness measures as an adjunct to consumer preference/acceptability evaluation. Food Quality and Preference, 7(3), 239-249.

Chakravorty, J., Ghosh, S., \& Meyer-Rochow, V. B. (2011). Practices of entomophagy and entomotherapy by members of the Nyishi and Galo tribes, two ethnic groups of the state of Arunachal Pradesh (North-East India). Journal of Ethnobiology and Ethnomedicine, 7, 5.

Chung, S.-J., \& Vickers, Z. (2007). Influence of sweetness on the sensory-specific satiety and long-term acceptability of tea. Food Quality and Preference, 18(2), 256-264.

Conner, M. T. (1994). An individualised psychological approach to measuring influences on consumer preferences. In H. J. H. MacFie \& D. M. H. Thomson (Eds.), Measurement of Food Preferences (pp. 167-201). Boston, MA: Springer US.

\section{D}

de Boer, J., Schösler, H., \& Boersema, J. J. (2013). Motivational differences in food orientation and the choice of snacks made from lentils, locusts, seaweed or "hybrid" meat. Food Quality and Preference, 28(1), 32-35.

DeFoliart, G. R. (1992). Insects as human food: Gene DeFoliart discusses some nutritional and economic aspects. Crop Protection, 11(5), 395-399.

Defoliart, G. R. (1997). An overview of the role of edible insects in preserving biodiversity. Ecology of Food and Nutrition, 36(2-4), 109-132.

Deroy, O., Reade, B., \& Spence, C. (2015). The insectivore's dilemma, and how to take the West out of it. Food Quality and Preference, 44, 44-55.

Dick, A., Chakravarti, D., \& Biehal, G. (1990). Memory-based inferences during consumer choice. Journal of Consumer Research, 82-93. 


\section{E}

Einstein, M. A., \& Hornstein, I. (1970). Food preferences of college students and nutritional implications. Journal of Food Science, 35(4), 429-436.

Elzerman, J. E., Hoek, A. C., van Boekel, M. A. J. S., \& Luning, P. A. (2011). Consumer acceptance and 240. appropriateness of meat substitutes in a meal context. Food Quality and Preference, 22(3), 233-

F

Fenko, A., Backhaus, B. W., \& van Hoof, J. J. (2015). The influence of product- and person-related factors on consumer hedonic responses to soy products. Food Quality and Preference, 41, 30-40.

Fessler, D. M., \& Navarrete, C. D. (2003). Meat is good to taboo: Dietary proscriptions as a product of the interaction of psychological mechanisms and social processes. Journal of Cognition and Culture, 3(1), 1-40.

Fieldhouse, P. (1996). Food and nutrition : customs and culture. Cheltenham: Nelson Thornes.

Frewer, L., Lassen, J., Kettlitz, B., Scholderer, J., Beekman, V., \& Berdal, K. G. (2004). Societal aspects of genetically modified foods. Food and Chemical Toxicology, 42(7), 1181-1193.

Frewer, L., Scholderer, J., \& Lambert, N. (2003). Consumer acceptance of functional foods: issues for the future. British Food Journal, 105(10), 714-731.

\section{G}

Garnett, T. (2011). Where are the best opportunities for reducing greenhouse gas emissions in the food system (including the food chain)? Food Policy, 36, S23-S32.

Garro, L. C. (2000). Remembering what one knows and the construction of the past: A comparison of cultural consensus theory and cultural schema theory. Ethos, 28(3), 275-319.

Ghaly, A. E., \& Alkoaik, F. (2009). The yellow mealworm as a novel source of protein. American Journal of Agricultural and Biological Sciences, 4(4), 319-331.

Giacalone, D., \& Jaeger, S. R. (2016). Better the devil you know? How product familiarity affects usage versatility of foods and beverages. Journal of Economic Psychology, 55, 120-138.

Gmuer, A., Guth, J. N., Hartmann, C., \& Siegrist, M. (2016). Effects of the degree of processing of insect ingredients in snacks on expected emotional experiences and willingness to eat. Food Quality and Preference, 54, 117-127.

Godfray, H. C. J., Beddington, J. R., Crute, I. R., Haddad, L., Lawrence, D., Muir, J. F., Pretty, J., Robinson, S., Thomas, S. M., \& Toulmin, C. (2010). Food security: the challenge of feeding 9 billion people. Science, 327(5967), 812-818.

Gordon, D. G. (2013). The eat-a-bug cookbook, revised: 40 ways to cook crickets, grasshoppers, ants, water bugs, spiders, centipedes, and their kin: Ten Speed Press.

Gorham, J. (1979). The significance for human health of insects in food. Annual Reviews in Entomology, 24(1), 209-224.

Gray, J., Gomaa, E., \& Buckley, D. (1996). Oxidative quality and shelf life of meats. Meat Science, 43, 111-123. 
Griskevicius, V., Cantú, S. M., \& Vugt, M. v. (2012). The evolutionary bases for sustainable behavior: Implications for marketing, policy, and social entrepreneurship. Journal of Public Policy \& Marketing, 31(1), 115-128.

Grunert, K. G. (2006). Future trends and consumer lifestyles with regard to meat consumption. Meat Science, 74(1), 149-160.

Grunert, K. G., Bredahl, L., \& Bruns $\varnothing$, K. (2004). Consumer perception of meat quality and implications for product development in the meat sector-a review. Meat Science, 66(2), 259-272.

Grunert, K. G., \& van Trijp, H. (2014). Consumer-Oriented New Product Development. Encyclopedia of Agriculture and Food Systems, 2, 375-386.

H

Halloran, A., Vantomme, P., Hanboonsong, Y., \& Ekesi, S. (2015). Regulating edible insects: the challenge of addressing food security, nature conservation, and the erosion of traditional food culture. Food Security, 7(3), 739-746.

Hanboonsong, Y. (2010). Edible insects and associated food habits in Thailand. Forest insects as food: humans bite back, 173-182.

Hanboonsong, Y., Jamjanya, T., \& Durst, P. B. (2013). Six-legged livestock: edible insect farming, collection and marketing in Thailand.

Harris, M. (1985). Good to eat: Riddles of food and culture: Simon and Schuster (pp 13-18, 154-174).

Hartmann, C., Shi, J., Giusto, A., \& Siegrist, M. (2015). The psychology of eating insects: A cross-cultural comparison between Germany and China. Food Quality and Preference, 44, 148-156.

Hoeffler, S. (2003). Measuring preferences for really new products. Journal of Marketing Research, 40(4), 406-420.

Hoek, A. C., Elzerman, J. E., Hageman, R., Kok, F. J., Luning, P. A., \& de Graaf, C. (2013). Are meat substitutes liked better over time? A repeated in-home use test with meat substitutes or meat in meals. Food Quality and Preference, 28(1), 253-263.

Hoek, A. C., Luning, P. A., Weijzen, P., Engels, W., Kok, F. J., \& de Graaf, C. (2011). Replacement of meat by meat substitutes. A survey on person- and product-related factors in consumer acceptance. Appetite, 56(3), 662-673.

Hofstede, G. (2001). Culture's consequences: Comparing values, behaviors, institutions and organizations across nations. California: Sage Publications.

Hofstede, G., \& McCrae, R. R. (2004). Personality and culture revisited: Linking traits and dimensions of culture. Cross-Cultural Research, 38(1), 52-88.

Honkanen, P., Olsen, S. O., \& Verplanken, B. (2005). Intention to consume seafood-the importance of habit. Appetite, 45(2), 161-168.

Hopkins, J. (2014). Strange Foods: Tuttle Publishing.

Horgen, K. B., \& Brownell, K. D. (2002). Comparison of price change and health message interventions in promoting healthy food choices. Health Psychology, 21(5), 505.

House, J. (2016). Consumer acceptance of insect-based foods in the Netherlands: Academic and commercial implications. Appetite, 107, 47-58. 
Jaeger, S. R., Andani, Z., Wakeling, I. N., \& MacFie, H. J. (1998). Consumer preferences for fresh and aged apples: a cross-cultural comparison. Food Quality and Preference, 9(5), 355-366.

Jaeger, S. R., \& Ares, G. (2014). Lack of evidence that concurrent sensory product characterisation using CATA questions bias hedonic scores. Food Quality and Preference, 35, 1-5.

Jaeger, S. R., Chheang, S. L., Yin, J., Bava, C. M., Gimenez, A., Vidal, L., \& Ares, G. (2013). Check-all-thatapply (CATA) responses elicited by consumers: Within-assessor reproducibility and stability of sensory product characterizations. Food Quality and Preference, 30(1), 56-67.

\section{K}

Kardes, F. R., Posavac, S. S., \& Cronley, M. L. (2004). Consumer inference: A review of processes, bases, and judgment contexts. Journal of Consumer Psychology, 14(3), 230-256.

Kellert, S. R. (1993). Values and perceptions of invertebrates. Conservation Biology, 7(4), 845-855.

Knodel, J. (1995). Focus groups as a qualitative method for crosscultural research in social gerontology. Journal of Cross-Cultural Gerontology, 10(1-2), 7-20.

Köster, E. P. (2009). Diversity in the determinants of food choice: A psychological perspective. Food Quality and Preference, 20(2), 70-82.

Krueger, R. A., \& Casey, M. A. (2000). Focus groups. A practical guide for applied research, 3.

L

Ladikos, D., \& Lougovois, V. (1990). Lipid oxidation in muscle foods: A review. Food Chemistry, 35(4), 295-314.

Lange, C., Martin, C., Chabanet, C., Combris, P., \& Issanchou, S. (2002). Impact of the information provided to consumers on their willingness to pay for Champagne: comparison with hedonic scores. Food Quality and Preference, 13(7), 597-608.

Lensvelt, E. J., \& Steenbekkers, L. (2014). Exploring consumer acceptance of entomophagy: a survey and experiment in Australia and The Netherlands. Ecology of Food and Nutrition, 53(5), 543-561.

Loewen, R., \& Pliner, P. (1999). Effects of prior exposure to palatable and unpalatable novel foods on children's willingness to taste other novel foods. Appetite, 32(3), 351-366.

Looy, H., Dunkel, F. V., \& Wood, J. R. (2014). How then shall we eat? Insect-eating attitudes and sustainable foodways. Agriculture and Human Values, 31(1), 131-141.

Looy, H., \& Wood, J. R. (2006). Attitudes toward invertebrates: Are educational "bug banquets" effective? The Journal of Environmental Education, 37(2), 37-48.

Luo, Z. Y. (1997). Insects as food in China. Ecology of Food and Nutrition, 36(2-4), 201-207.

\section{M}

MacClancy, J., Henry, C. J., \& Macbeth, H. (2009). Consuming the inedible: neglected dimensions of food choice (Vol. 6, pp.1-16): Berghahn Books. 
Malcolmson, L., Vaisey-Genser, M., Przybylski, R., \& Eskin, N. (1994). Sensory stability of canola oil: present status of shelf life studies. Journal of the American Oil Chemists' Society, 71(4), 435-440.

Mandler, G. (1982). The structure of value: Accounting for taste. In Clark, M.S. \& Fiske, S.T. (Eds.), Affect and Cognition (pp. 3-36): Hillsdale, NJ: Lawrence Erlbaum.

Martins, Y., Pelchat, M. L., \& Pliner, P. (1997). "Try it, it's good and it's good for you": Effects of taste and nutrition information on willingness to try novel foods. Appetite, 28(2), 89-102.

Martins, Y., \& Pliner, P. (2005). Human food choices: An examination of the factors underlying acceptance/rejection of novel and familiar animal and nonanimal foods. Appetite, 45(3), 214224.

Martins, Y., \& Pliner, P. (2006). "Ugh! That's disgusting!": Identification of the characteristics of foods underlying rejections based on disgust. Appetite, 46(1), 75-85.

Meiselman, H. L. (2003). A three-factor approach to understanding food quality: the product, the person and the environment*. Food Service Technology, 3(3-4), 99-105.

Mela, D. J. (2001a). Determinants of food choice: relationships with obesity and weight control. Obesity Research, 9(S11), 249S-255S.

Mela, D. J. (2001b). Why do we like what we like? Journal of the Science of Food and Agriculture, 81(1), 10-16.

Menzel, P., \& d'Aluisio, F. (1998). Man eating bugs: The art and science of eating insects. New York: Ten Speed Press.

Meyer-Rochow, V. (2009). Food taboos: their origins and purposes. Journal of Ethnobiology and Ethnomedicine, 5.

Mitsuhashi, J. (1997). Insects as traditional foods in Japan. Ecology of Food and Nutrition, 36(2-4), 187-199.

Morgan, D. L., \& Spanish, M. T. (1984). Focus groups: A new tool for qualitative research. Qualitative sociology, 7(3), 253-270.

\section{N}

Nam, K.-C., Jo, C., \& Lee, M. (2010). Meat products and consumption culture in the East. Meat Science, 86(1), 95-102.

Napolitano, F., Braghieri, A., Piasentier, E., Favotto, S., Naspetti, S., \& Zanoli, R. (2010). Effect of information about organic production on beef liking and consumer willingness to pay. Food Quality and Preference, 21(2), 207-212.

Napolitano, F., Castellini, C., Naspetti, S., Piasentier, E., Girolami, A., \& Braghieri, A. (2013). Consumer preference for chicken breast may be more affected by information on organic production than by product sensory properties. Poultry Science, 92(3), 820-826.

Nicklaus, S., \& Issanchou, S. (2006). Children and food choice. In Frewer, L.J. \& van Trijp, H.C.M. (Eds.), Understanding consumers of food products (pp. 329-358), Cambridge: Woodhead Publishing.

\section{0}

Obopile, M., \& Seeletso, T. G. (2013). Eat or not eat: an analysis of the status of entomophagy in Botswana. Food Security, 5(6), 817-824. 
Olson, J. C. (1978). Inferential Belief Formation in the Cue Utilization Process. In H. K. Hunt (Ed.), Advances in Consumer Research (Vol. 5, pp. 706-713). Ann Arbor, MI: Association for Consumer Research.

Oppermann, A., de Graaf, C., Scholten, E., Stieger, M., \& Piqueras-Fiszman, B. (2017). Comparison of Rate-All-That-Apply (RATA) and Descriptive sensory Analysis (DA) of model double emulsions with subtle perceptual differences. Food Quality and Preference, 56, 55-68.

Ottenbacher, M., \& Harrington, R. J. (2008). The culinary innovation process: A study of Michelinstarred chefs. Journal of Culinary Science \& Technology, 5(4), 9-35.

\section{P}

Pelchat, M. L., \& Pliner, P. (1995). "Try it. You'll like it". Effects of information on willingness to try novel foods. Appetite, 24(2), 153-165.

Peracchio, L. A., \& Tybout, A. M. (1996). The moderating role of prior knowledge in schema-based product evaluation. Journal of Consumer Research, 177-192.

Perrea, T., Grunert, K. G., \& Krystallis, A. (2015). Consumer Value perceptions of food products from emerging processing technologies: A cross-cultural exploration. Food Quality and Preference, 39, 95-108.

Piqueras-Fiszman, B., \& Spence, C. (2015). Sensory expectations based on product-extrinsic food cues: an interdisciplinary review of the empirical evidence and theoretical accounts. Food Quality and Preference, 40, 165-179.

Pliner, P. (1982). The effects of mere exposure on liking for edible substances. Appetite, 3(3), 283-290.

Pliner, P., \& Hobden, K. (1992). Development of a scale to measure the trait of food neophobia in humans. Appetite, 19(2), 105-120.

Pliner, P., Pelchat, M., \& Grabski, M. (1993). Reduction of neophobia in humans by exposure to novel foods. Appetite, 20(2), 111-123.

Pliner, P., \& Pelchat, M. L. (1991). Neophobia in humans and the special status of foods of animal origin. Appetite, 16(3), 205-218.

Pliner, P., \& Stallberg-White, C. (2000). "Pass the ketchup, please": familiar flavors increase children's willingness to taste novel foods. Appetite, 34(1), 95-103.

Prescott, J. (1998). Comparisons of taste perceptions and preferences of Japanese and Australian consumers: overview and implications for cross-cultural sensory research. Food Quality and Preference, 9(6), 393-402.

Prescott, J., \& Bell, G. (1995). Cross-cultural determinants of food acceptability: recent research on sensory perceptions and preferences. Trends in Food Science \& Technology, 6(6), 201-205.

Prescott, J., Young, O., O'Neill, L., Yau, N., \& Stevens, R. (2002). Motives for food choice: a comparison of consumers from Japan, Taiwan, Malaysia and New Zealand. Food Quality and Preference, 13(7), 489-495.

Prescott, J., Young, O., Zhang, S., \& Cummings, T. (2004). Effects of added "flavour principles" on liking and familiarity of a sheepmeat product: a comparison of Singaporean and New Zealand consumers. Food Quality and Preference, 15(2), 187-194. 
Ramos-Elorduy, J. (1997). The importance of edible insects in the nutrition and economy of people of the rural areas of Mexico. Ecology of Food and Nutrition, 36(5), 347-366.

Ramos-Elorduy, J., Moreno, J. M. P., Prado, E. E., Perez, M. A., Otero, J. L., \& De Guevara, O. L. (1997). Nutritional value of edible insects from the state of Oaxaca, Mexico. Journal of Food Composition and Analysis, 10(2), 142-157.

Rappoport, L. H., Peters, G. R., Huff-Corzine, L., \& Downey, R. G. (1992). Reasons for eating: an exploratory cognitive analysis. Ecology of Food and Nutrition, 28(3), 171-189.

Ratneshwar, S., \& Shocker, A. D. (1991). Substitution in use and the role of usage context in product category structures. Journal of Marketing Research, 281-295.

Raudenbush, B., \& Frank, R. A. (1999). Assessing food neophobia: The role of stimulus familiarity. Appetite, 32(2), 261-271.

Resurreccion, A. V. (2007). Consumer sensory testing for food product development. In Brody, A. L. \& Lord, J.B. (Eds.), Developing New Food Products for a Changing Marketplace (pp. 365-406), Boca Raton, Fl: Taylor \& Francis Group.

Roininen, K., Lahteenmaki, L., \& Tuorila, H. (1999). Quantification of consumer attitudes to health and hedonic characteristics of foods. Appetite, 33(1), 71-88.

Rozin, P. (1996). The socio-cultural context of eating and food choice. In Meiselman, H.L. \& MacFie, H.F.H. (Eds.), Food choice, acceptance and consumption (pp. 83-104), London: Chapman \& Hall.

Rozin, P. (2003). Five potential principles for understanding cultural differences in relation to individual differences. Journal of Research in Personality, 37(4), 273-283.

Rozin, P. (2006). The integration of biological, social, cultural and psychological influences on food choice. Frontiers in nutritional science, 3, 19.

Rozin, P., \& Fallon, A. (1980). The psychological categorization of foods and non-foods: A preliminary taxonomy of food rejections. Appetite, 1(3), 193-201.

Rozin, P., \& Fallon, A. (1987). A perspective on disgust. Psychological Review, 94(1), 23.

Rozin, P., \& Vollmecke, T. A. (1986). Food Likes and Dislikes. Annual Review of Nutrition, 6, 433-456.

Ruark, A., Vingerhoeds, M. H., Kremer, S., Nijenhuis-de Vries, M. A., \& Piqueras-Fiszman, B. (2016). Insights on older adults' perception of at-home sensory-hedonic methods: A case of Ideal Profile Method and CATA with ideal. Food Quality and Preference, 53, 29-38.

Ruby, M., Rozin, P., \& Chan, C. (2015). Determinants of willingness to eat insects in the USA and India. Journal of Insects as Food and Feed, 1(3), 215-225.

Rumpold, B. A., \& Schlüter, O. K. (2013). Potential and challenges of insects as an innovative source for food and feed production. Innovative Food Science \& Emerging Technologies, 17, 1-11.

\section{S}

Sabbe, S., Verbeke, W., Deliza, R., Matta, V., \& Van Damme, P. (2009). Effect of a health claim and personal characteristics on consumer acceptance of fruit juices with different concentrations of açaí (Euterpe oleracea Mart.). Appetite, 53(1), 84-92.

Schickenberg, B., Van Assema, P., Brug, J., \& De Vries, N. (2008). Are the Dutch acquainted with and willing to try healthful food products? The role of food neophobia. Public Health Nutrition, 11(05), 493-500. 
Schifferstein, H. (2001). Effects of product beliefs on product perception and liking. In Frewer, L.J., Risvik, E., \& Schifferstein, H. (Eds.) Food, People and Society: A European Perspective of Consumers' Food Choice (pp. 73-96): Springer.

Schösler, H., de Boer, J., \& Boersema, J. J. (2012). Can we cut out the meat of the dish? Constructing consumer-oriented pathways towards meat substitution. Appetite, 58(1), 39-47.

Schouteten, J., J. , De Steur, H., De Pelsmaeker, S., Lagast, S., Juvinal, J. G., De Bourdeaudhuij, I., Verbeke, W., \& Gellynck, X. (2016). Emotional and sensory profiling of insect-, plant- and meatbased burgers under blind, expected and informed conditions. Food Quality and Preference, 52, 27-31.

Schutz, H. G., \& Martens, M. (2001). Appropriateness as a cognitive-contextual measure of food attitudes. In Frewer, L.J., Risvik, E., \& Schifferstein, H. (Eds.) Food, People and Society: A European Perspective of Consumers' Food Choice (pp. 247-266): Springer.

Seaton, R. W., \& Gardner, B. W. (1959). Acceptance measurement of unusual foods. Journal of Food Science, 24(3), 271-278.

Setchfield, N. (2010). Yuck! The things people eat. London: Merrell Publishers.

Shelomi, M. (2015). Why we still don't eat insects: Assessing entomophagy promotion through a diffusion of innovations framework. Trends in Food Science \& Technology, 45(2), 311-318.

Shepherd, R. (2001). Does taste determine consumption? Understanding the psychology of food choice. In Frewer, L.J., Risvik, E., \& Schifferstein, H. (Eds.) Food, People and Society: A European Perspective of Consumers' Food Choic (pp. 117-130), Berlin: Springer.

Sidel, J. L., \& Stone, H. (1993). The role of sensory evaluation in the food industry. Food Quality and Preference, 4(1), 65-73.

Siemianowska, E., Kosewska, A., Aljewicz, M., Skibniewska, K. A., Polak-Juszczak, L., Jarocki, A., \& Jedras, M. (2013). Larvae of mealworm (Tenebrio molitor L.) as European novel food. Agricultural Sciences, 4(6), 287.

Siro, I., Kapolna, E., Kapolna, B., \& Lugasi, A. (2008). Functional food. Product development, marketing and consumer acceptance-A review. Appetite, 51(3), 456-467.

Sobal, J., \& Bisogni, C. A. (2009). Constructing food choice decisions. Annals of Behavioral Medicine, 38, S37-S46.

Solheim, R., \& Lawless, H. T. (1996). Consumer purchase probability affected by attitude towards low-fat foods, liking, private body consciousness and information on fat and price. Food Quality and Preference, 7(2), 137-143.

Southgate, D. A. (1996). Dietary change: changing patterns of eating. In Meiselman, H.L. \& MacFie, H.F.H. (Eds.), Food choice, acceptance and consumption (pp. 365-391), London: Chapman \& Hall.

Spreng, R. A., \& Page, T. J. (2001). The impact of confidence in expectations on consumer satisfaction. Psychology \& Marketing, 18(11), 1187-1204.

Stallberg-White, C., \& Pliner, P. (1999). The effect of flavor principles on willingness to taste novel foods. Appetite, 33(2), 209-221.

Steenkamp, J.-B. E. (1990). Conceptual model of the quality perception process. Journal of Business Research, 21(4), 309-333.

Steenkamp, J.-B. E., \& van Trijp, H. C. (1996). Quality guidance: a consumer-based approach to food quality improvement using partial least squares. European Review of Agricultural Economics, 23(2), 195-215.

Stein, L. J., Nagai, H., Nakagawa, M., \& Beauchamp, G. K. (2003). Effects of repeated exposure and health-related information on hedonic evaluation and acceptance of a bitter beverage. Appetite, 40(2), 119-129. 
Steinfeld, H., Gerber, P., Wassenaar, T., Castel, V., \& de Haan, C. (2006). Livestock's long shadow: environmental issues and options: Food \& Agriculture Organisation.

Steptoe, A., Pollard, T. M., \& Wardle, J. (1995). Development of a measure of the motives underlying the selection of food: the food choice questionnaire. Appetite, 25(3), 267-284.

Strickland, C. (1999). Conducting focus groups cross-culturally: Experiences with pacific northwest Indian people. Public Health Nursing, 16(3), 190-197.

\section{T}

Tepper, B. J., \& Trail, A. C. (1998). Taste or health: A study on consumer acceptance of corn chips. Food Quality and Preference, 9(4), 267-272.

Threlfall, K. D. (1999). Using focus groups as a consumer research tool. Journal of Marketing Practice: Applied Marketing Science, 5(4), 102-105.

Tilman, D., Balzer, C., Hill, J., \& Befort, B. L. (2011). Global food demand and the sustainable intensification of agriculture. Proceedings of the National Academy of Sciences, 108(50), 2026020264.

Tobler, C., Visschers, V. H., \& Siegrist, M. (2011). Eating green. Consumers' willingness to adopt ecological food consumption behaviors. Appetite, 57(3), 674-682.

Trope, Y., Liberman, N., \& Wakslak, C. (2007). Construal levels and psychological distance: Effects on representation, prediction, evaluation, and behavior. Journal of consumer psychology: the official journal of the Society for Consumer Psychology, 17(2), 83.

Tucker, C. A. (2014). The significance of sensory appeal for reduced meat consumption. Appetite, 81, 168-179.

Tuorila, H. (2007). Sensory perception as a basis of food acceptance and consumption. In Macfie, H.F.H. (Ed.), Consumer-led food product development (pp. 34-65), Cambridge: Woodhead Publishing.

Tuorila, H., Andersson, Å., Martikainen, A., \& Salovaara, H. (1998). Effect of product formula, information and consumer characteristics on the acceptance of a new snack food. Food Quality and Preference, 9(5), 313-320.

Tuorila, H., \& Cardello, A. V. (2002). Consumer responses to an off-flavor in juice in the presence of specific health claims. Food Quality and Preference, 13(7), 561-569.

Tuorila, H., Lahteenmaki, L., Pohjalainen, L., \& Lotti, L. (2001). Food neophobia among the Finns and related responses to familiar and unfamiliar foods. Food Quality and Preference, 12(1), 29-37.

Tuorila, H., \& MacFie, H. (2007). Sensory perception as a basis of food acceptance and consumption. Consumer-led food product development, 34-65.

Tuorila, H., Meiselman, H. L., Bell, R., Cardello, A. V., \& Johnson, W. (1994). Role of sensory and cognitive information in the enhancement of certainty and liking for novel and familiar Foods. Appetite, 23(3), 231-246.

Tuorila, H., Meiselman, H. L., Cardello, A. V., \& Lesher, L. L. (1998). Effect of expectations and the definition of product category on the acceptance of unfamiliar foods. Food Quality and Preference, 9(6), 421-430.

Turmo, I. G. (2009). The Concepts of Food and Non-Food: Perspectives from Spain. In MacClancy J., Henry, C.J., MacBeth, H. (Ed.), Consuming the Inedible: Neglected Dimensions of Food Choice (Vol. 6, pp. 43-52): Berghahn Books. 
Urala, N., \& Lähteenmäki, L. (2004). Attitudes behind consumers' willingness to use functional foods. Food Quality and Preference, 15(7), 793-803.

\section{V}

van Huis, A. (2013). Potential of Insects as Food and Feed in Assuring Food Security. Annual Reviews in Entomology, 58, 563-583.

van Huis, A., van Gurp, H., \& Dicke, M. (2012). Het insectenkookboek. Amsterdam [etc.]: Atlas.

van Huis, A., van Itterbeeck, J., Klunder, H., Mertens, E., Halloran, A., Muir, G., \& Vantomme, P. (2013). Edible insects: future prospects for food and feed security: Food and agriculture organization of the United nations (FAO).

van Trijp, H. C. M., \& Fischer, A. R. H. (2011). Mobilizing consumer demand for sustainable development The TransForum Model: Transforming Agro Innovation Toward Sustainable Development (pp. 7396): Springer.

van Trijp, H. C. M., \& Steenkamp, J. E. B. M. (2005). Consumer-oriented new product development: principles and practice. Innovation in agri-food systems, 87-124.

van Trijp, H. C. M., \& van Kleef, E. (2008). Newness, value and new product performance. Trends in Food Science \& Technology, 19(11), 562-573.

Vanhonacker, F., Van Loo, E. J., Gellynck, X., \& Verbeke, W. (2013). Flemish consumer attitudes towards more sustainable food choices. Appetite, 62, 7-16.

Varela, P., \& Ares, G. (2012). Sensory profiling, the blurred line between sensory and consumer science. A review of novel methods for product characterization. Food Research International, 48(2), 893908.

Verbeke, W. (2005). Consumer acceptance of functional foods: socio-demographic, cognitive and attitudinal determinants. Food Quality and Preference, 16(1), 45-57.

Verbeke, W. (2006). Functional foods: Consumer willingness to compromise on taste for health? Food Quality and Preference, 17(1), 126-131.

Verbeke, W. (2015). Profiling consumers who are ready to adopt insects as a meat substitute in a Western society. Food Quality and Preference, 39, 147-155.

Vermeir, I., \& Verbeke, W. (2006). Sustainable food consumption: Exploring the consumer "attitudebehavioral intention" gap. Journal of Agricultural and Environmental ethics, 19(2), 169-194.

Verneau, F., La Barbera, F., Kolle, S., Amato, M., Del Giudice, T., \& Grunert, K. (2016). The effect of communication and implicit associations on consuming insects: An experiment in Denmark and Italy. Appetite 106, 30-36.

Vickers, Z., \& Holton, E. (1998). A comparison of taste test ratings, repeated consumption, and postconsumption ratings of different strengths of iced tea. Journal of Sensory Studies, 13(2), 199212.

Wansink, B. (2002). Changing eating habits on the home front: Lost lessons from World War II research. Journal of Public Policy \& Marketing, 90-99. 
W

Westenhoefer, J., \& Pudel, V. (1993). Pleasure from food: Importance for food choice and consequences of deliberate restriction. Appetite 20(3), 246-249.

Woodruff, R. B., Cadotte, E. R., \& Jenkins, R. L. (1983). Modeling consumer satisfaction processes using experience-based norms. Journal of Marketing Research, 296-304.

Worch, T., \& Punter, P. (2015). Ideal profiling as a sensory profiling technique. In Delarue, J., Lawlor, B., \& Rogeaux, M., Rapid Sensory Profiling Techniques: Applications in New Product Development and Consumer Research (pp. 307-332), Cambridge: Woodhead Publishing.

Y

Yates-Doerr, E. (2012). Meeting the demand for meat? Anthropology Today, 28(1), 11-15. Yen, A. L. (2009). Edible insects: Traditional knowledge or western phobia? Entomological Research, 39(5), 289-298.

Yeomans, M. R., Chambers, L., Blumenthal, H., \& Blake, A. (2008). The role of expectancy in sensory and hedonic evaluation: The case of smoked salmon ice-cream. Food Quality and Preference, 19(6), 565-573.

Yhoung-Aree, J., Puwastien, P., \& Attig, G. A. (1997). Edible insects in Thailand: An unconventional protein source? Ecology of Food and Nutrition, 36(2-4), 133-149.

Yi, L., Lakemond, C. M., Sagis, L. M., Eisner-Schadler, V., van Huis, A., \& van Boekel, M. A. (2013). Extraction and characterisation of protein fractions from five insect species. Food Chemistry, 141(4), 3341-3348.

\section{Z}

Zajonc, R. B. (1968). Attitudinal effects of mere exposure. Personality and Social Psychology Bulletin, 9(2 pt. 2), 1-27. 

SUMMARY 
Growing global food demands have raised concerns about food security, where the severe environmental impact of large scale livestock production has increased the need for more sustainable sources of protein. In recent years, edible insects have gained attention due to their nutritional and environmental advantages over conventional meat. While numerous species of edible insects are enjoyed in various cultures around the world, most Western consumers react with disgust and aversion towards eating creatures that are more commonly regarded as filth than as food. The low consumer acceptance of this culturally inappropriate food is currently considered to be one of the key barriers to attaining the benefits of this potentially good source of food. This thesis explores the various factors that contribute to the acceptance (and rejection) of culturally inappropriate foods to provide insights into how insects may gain entry into Western diets.

The objective of this thesis was to investigate what determines the consumer acceptance of culturally inappropriate foods. This thesis extends the current body of literature that mostly focuses on the psychological determinants by giving more attention to the roles of culture, taste and product dimensions. Using cross-cultural focus groups, hedonic and descriptive sensory studies, and systematically-varied product properties, an interdisciplinary approach was employed to provide new insights into the consumer acceptance and product development of culturally inappropriate foods like insects.

The role of culture in determining acceptance was first explored using stage-wise focus group discussions (Chapter 2) to examine how consumer perceptions and rationales differ across two cultural settings-one where insects are part of the local diet (Thailand), and one where insects are generally not regarded as food (The Netherlands). It was found that the key difference between those who accept versus reject insects as food lies in individual experiences. People from both cultures who lacked taste experience with a certain edible insect species were generally reluctant to eat it, giving reasons relating to the physical appearances and speciesrelated associations. These negative associations were only triggered when the food had not been eaten before. Culture influences acceptance by providing the environment within which locals gain experiences with obtaining, preparing and consuming the food. This resulted not only in Thai eaters gaining extensive knowledge regarding the taste and usage of available species, it also contributed to culture-specific preferences for certain species and products. The lack of cultural exposure therefore creates additional challenges for Dutch consumers to accept insects as food, where the unfamiliar taste, low availability, high price and lack of knowledge regarding its use remain barriers to acceptance.

The role of taste in determining acceptance was investigated in the next chapters of the thesis (Chapters 3 and 4). Through hedonic and descriptive sensory evaluations of beef burger patties claimed to contain unusual novel foods (i.e. lamb brain, frog meat, mealworms), the influence of the food's identity (i.e. label) and properties (i.e. recipe) on acceptance were examined. In 
chapter 3, it was found that for culturally unusual foods like insects, the low willingness to eat was heavily influenced by its perceived inappropriateness as food. Consumers may like the taste of a novel food upon trying it, but remain unwilling to eat it again if they do not consider it to be appropriate as food.

In chapter 4, descriptive sensory profiling revealed that Dutch consumers had little prior knowledge of the taste of the unusual novel foods and expected many negative attributes that were related to associations with the item (e.g. mealy mealworms, mushy lamb brains). After tasting, it was the properties of the food that mainly determined the taste experience. Although food appropriateness determined the willingness to eat, the food's taste still plays a role in influencing food appropriateness. Together, chapters 3 and 4 conclude that introducing products containing unusual novel foods like insects could benefit from developing products that disconfirm the expected negative attributes that are associated with the novel food items, but the low food appropriateness means that other efforts are required to encourage repeated consumption.

The role of product properties in determining acceptance was examined in the last two studies using systematically varied mealworm products (Chapters 5 and 6). Results of chapter 5 showed that Dutch consumers had a strong preference for savoury products with ground mealworms despite their unfamiliarity with eating mealworms. Consequently they evaluated those products more positively in product appropriateness, expected sensory-liking, and willingness to buy and try, but even the most appropriate mealworm products were inferior to the original mealwormfree products. This poses difficulties when insect-based foods have to compete against existing products on the market. A more appropriate product nevertheless increases the chance of trial and acceptance by reducing the negative impact of the mealworms on the product. Food neophobia and demographic variables (e.g. age, gender, education level) were found to mainly affect the willingness to try, and had little influence on the other measures.

Chapter 6 showed that a more appropriate product improves the expected sensory-liking and willingness to buy mealworm products once and regularly. However, for a more appropriate product to improve consumption intentions, consumers should already be motivated to eat insects. Descriptive and ideal sensory profiling revealed that mealworm products were preferred to taste similar to the original products but were expected and experienced to taste different. The experienced taste and product appropriateness were found to contribute significantly to the willingness to buy mealworm products regularly. Nevertheless, even for willing consumers, intentions to regularly consume insect-based foods were still hindered by other practical and socio-cultural barriers (e.g. price, knowledge, availability, social acceptance).

Chapter 7 concludes this thesis with an overview of the main findings and a discussion of its contributions to understanding the consumer acceptance of culturally inappropriate foods. New insights revealed the complex challenges faced in the consumer acceptance and product 
development of culturally new foods that are not only unfamiliar but also perceived to be inappropriate for consumption. This chapter discusses the importance of culture in determining food choices and the limited role of food neophobia and demographic variables. In addition, it stresses the importance of distinguishing between liking the taste of a food and liking to eat a food, and between trying a food out of curiosity and eating it on a regular basis. The chapter addresses the dilemmas when developing products from culturally inappropriate foods, where little is known about its taste and how it should be prepared, and where the presence of an inappropriate food is sometimes sufficient to cause rejection. These findings are further discussed in relation to the case of introducing insects as food in Western cultures. The difficulties of improving consumer acceptance, of undertaking product development, and of achieving a reduction of meat consumption through eating insects are critically evaluated.

In conclusion, achieving consumer acceptance of a culturally inappropriate food faces numerous challenges that extend beyond the initial psychological barriers of consuming a novel food. This thesis highlights the cultural, taste, and product dimensions that need to be addressed when a new source of food is to be introduced successfully into an existing diet. By focusing on the case of insects, we learned that to attain Western consumer acceptance, communicating the benefits of eating a food might help to raise interest but is insufficient to establish real consumption. Much more needs to be done not only in encouraging trial and changing consumer perceptions regarding the food's qualities. There is also a need to create a conducive environment that makes eating insects convenient, affordable, socially desirable, and enjoyable, all the while keeping sight of the original environmental goals of introducing insects as food in the West. 


\section{ACKNOWLEDGMENTS}


So here I am, standing by the finish line and thinking of all the great people who have inspired, challenged, taught, and encouraged me along the way. I am truly grateful to all of you for contributing to the completion of this thesis in so many ways.

First of all, I would like to thank my PhD supervisors Markus Stieger, Arnout Fischer and Hans van Trijp, for guiding me through this journey of discovery. Hans, every meeting with you has been extremely enlightening, I have never left your office without fresh insights. Arnout, without your help and philosophical discussions, I would not have dared to take the leap into the social sciences. Markus, you were more than a supervisor to me, you were a great mentor and friend who was always present to steer me in a good direction, lifting my spirits up when I'm down, and urging me to seize every opportunity to grow. I do hope we can team up again someday!

I would also like to take this opportunity to thank Catriona Lakemond for setting up and being the project leader of this interdisciplinary project, and to Marian Peters from VENIK and Arnold van Huis from the entomology department, for being incredibly supportive and generous with sharing their knowledge. I am grateful to the many other researchers of entomophagy for being great sources of inspiration, and to the reviewers whose valuable insights helped improve the papers tremendously.

Members of my reading committee: Prof Tiny van Boekel, Prof Cor van der Weele, Prof Wim Verbeke, and Dr Michael Bom Frost, thank you very much for taking the time to review this thesis and for coming to the defence.

To my previous supervisors and mentors, you were the ones who ignited my passion for research, which eventually led me to do a PhD. I am especially grateful to the wonderful NUSFST family in Singapore for first getting me addicted to studying the food sciences.

I thank Patcharaporn Tinchan too for a marvellous collaboration in the first study, and for playing a key role in ensuring the success of the cross-cultural work. Ning, my dear friend, I am honoured to have had the chance to know you and to team up with you, I fondly recall the times working together first over skype, then in Sakon Nakhon and in Bangkok!

Much thanks to the staff and students of Department of Food Technology and Nutrition, Kasetsart University Chalermphrakiat Sakon Nakhon Province Campus for their warm hospitality and assistance, as well as the Thai friends in Wageningen for embracing me into their community. $P$ Noo, P Noy, Kunalai, Noon, Good, Boat, Off, Plub, and many others, thank you all for helping to set up the collaboration, study materials, translations, cultural immersion, and for spoiling me with the most delicious Thai cooking!

Big thanks to Bea Steenbekkers, Bea, even though you were not part of my supervision team, you were always ready to help. I was very lucky to have you by my side to help me with each step of the focus group research. Even when that was done, you continued to be a great pillar of support throughout my PhD. 
To my four dedicated masters students whom I supervised - Qing Chen, Claudia Tibboel, Eva van den Berg and Yoeri Verbaan - I am very proud of you and you deserve to be proud too for the work you have achieved. You were more my accomplices than my students, and I enjoyed working hard, laughing hard, and learning together with you! I hope you've forgiven me by now for all the late nights that you had to endure.

Behind the scenes of the research there were many friends who helped with flipping the thousand burger patties, picking participants off the streets, and taste testing the products of the studies. Thanks Anika, Marie-Luise, Cho, Margo, Radhika, Melanie, Elisa, Sophie, Sarah, Renske, Marine, and Sara, for being my sidekicks and guinea pigs. I thank too the hundreds of enthusiastic participants who not only enabled the research, but whose eagerness to share their perspectives triggered many ideas.

Of course, a PhD journey is not only about doing the research. It is thanks to my colleagues and friends from Food Quality and Design (FQD), Marketing and Consumer Behaviour (MCB), and Human Nutrition (HNE) that life was especially colourful and enriching.

I've been very fortunate to be part of the fun-loving and caring FQD family - I can't thank you all enough for being such wonderful colleagues! I am grinning as I look back on our amusing lab trips, celebratory dinners, the big Singapore-Thailand adventure, and all the silliness and laughter we've shared day-in-day-out. Especially those whom I have spent a good deal of time in an office with, Van-Anh, Dieuwerke, Cho, George, Renske, Marine, Sara, Ita, Fahui, Chunyue, you guys made it all especially meaningful and memorable. A special thank you to Lysanne and Kimberley who took care of all the administrative matters, always with bright sunny smiles that never fail to light up my day. To Vincenzo, Andrijana, Bea, Ruud, Teresa, Eduardo, Matthijs, Geraldine, Daphne, Klementina, Daylan, and everyone else in FQD, thank you for your constant encouragements, especially when I most needed it.

And here a dedicated paragraph to thank my extraordinary bug family -- Bug 1 (Liya), Bug 3 (Renske) and Bug 4 (Faith), let us continue to invade and conquer the world of humans with the resilient spirit of cockroaches! Bug 1 and Bug 3, I couldn't be happier that you will be standing by my side as paranymphs.

To the energetic MCB group, thank you for all the great memories of WeDays, annual day outs, and 'Christmas' dinners that were always 'better late than never'. I have learned so much from all of you, particularly through the regular lunch clubs and PhD days. Kunalai, Robert, Lotte, Alex, Colin, Roxy, Luz, Betina, Andres, and many others, it's always so nice talking to all of you that a 'short' visit to the MCB corridor often ends up being a day's affair with many new inspirations and answers. Betina and Andres, I'm especially grateful for all your advices, when I'm lost in doubt, speaking to you always helps! And Ellen, you've been a great secretary to the group, thank you for helping me with my every request! 
To my HNE colleagues from the sensory science group, I am thankful for the experiences we have shared at sensory science courses and international conferences. Special thanks to Dione and Astrid for arranging the kitchen facilities and access to participant databases, which was an enormous help for the sensory studies.

I am immensely grateful to the ENLP community, a very brilliant group of future leaders in nutrition who have greatly inspired me and helped me to realize the importance of embracing my strengths and weaknesses.

Wendy, sweet neighbour, we've shared all our ups and downs, anxieties and ambitions together since we were teens. No words can describe how special that is to me! Dear Anika, there are a million things I have to thank you for, for inspiring me with your convictions, for pretzel deliveries, and for being a great friend! Thank you Georgina, and Ronald, for all the wonderful musical times that gave me a lot of comfort, and my squash team for the fighting spirit and exciting highs of the past four seasons. And Liesbeth, you've been a great friend and advisor from squash court to Leeuwenborch! To all my other friends and housemates whom I have not mentioned here, I want you to know that I am very grateful too for your friendship and for helping to preserve my sanity through this rollercoaster ride.

Last but not least, my family, Alvan, Wenjie, and especially my mommy and daddy, thank you for your unconditional love and support, and for giving me the strength and freedom to pursue my ambitions in a faraway land. Family Tempelman, thank you for giving me the warmth of a family here in the Netherlands!

Dearest Barend, my best friend and soulmate, thank you for making me laugh every day, for being my pillar of strength, and for pulling me through the difficult times of illness and stress. You may deny it again, but I still say it - I couldn't have done this without you!

Thank you all, I'm really happy to have made this journey with all of you!

\section{Grace}


ABOUT THE AUTHOR 



\section{CURRICULUM VITAE}

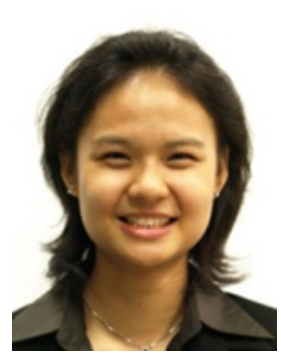

Hui Shan Grace Tan was born on 14th January 1986 in Singapore and spent most of her childhood in Japan and Hong Kong. In 2005, her first job as a baker inspired her to pursue a bachelors education in Food Science \& Technology at the National University of Singapore, which included a semester at University of California (Davis), and a semester as flavour applications intern at Givaudan (Singapore). She graduated with 1st class honours in 2009, and enrolled in a master's programme in sensory science at Wageningen University and University of Copenhagen. She did her masters internship in flavour chemistry at the Nestlé Research Centre (Lausanne, Switzerland) and graduated cum laude in 2011. She was awarded the Unilever Research Prize and the NVVL Prize for her thesis on the 'Pine Nut Syndrome', which investigated the source and mechanisms of the delayed taste disturbance caused by pine nuts.

In 2012, she started her PhD research on the consumer acceptance of insects as food at the departments of Food Quality \& Design, Division of Human Nutrition, and the Marketing \& Consumer Behaviour Group. Besides her research activities, she supervised practicals and masters theses, gave lectures, organized a 2-week PhD study tour to Singapore and Thailand, and presented her work at various international conferences. For her interdisciplinary research, she won the European Sensory Science Society student award at the 6th European Conference for Sensory and Consumer Research and the Rick Bell Memorial Scholarship at the 11th Pangborn Sensory Science Symposium.

Since February 2017, she works as scientific knowledge manager at Danone Nutricia Research, where she keeps up with the latest science, trends and products in medical nutrition for rare metabolic diseases and epilepsy. 


\section{PUBLICATIONS IN PEER-REVIEWED JOURNALS}

Tan, H.S.G., Tibboel, C.J., \& Stieger, M. (2017). Why do unusual foods like insects lack sensory appeal? Exploring the underlying sensory perceptions. Food Quality and Preference 60, 48-58.

Tan, H.S.G., Verbaan, Y.T., \& Stieger, M. (2017). How do better products improve the sensoryliking and willingness to buy insect-based foods? Food Research International 92, 95-105.

Tan, H.S.G., van den Berg, E., \& Stieger, M. (2016). The influence of product preparation, familiarity and individual traits on the consumer acceptance of insects as food. Food Quality and Preference 52, 222-231.

Tan, H.S.G., Fischer, A.R.H., van Trijp, H. C.M., \& Stieger, M. (2016). Tasty but nasty? Exploring the role of sensory-liking and food appropriateness in the willingness to eat unusual novel foods like insects. Food Quality and Preference 48, 293-302.

Evans, J., Alemu, M. H., Flore, R., Frøst, M. B., Halloran, A., Jensen, A. B., Maciel-Vergara, G., Meyer-Rochow, V.B., Münke-Svendsen,C., Olsen, S.B., Payne, C., Roos, N., Rozin, P., Tan, H.S.G., van Huis, A., Vantomme, P, \& Eilenberg, J. (2015). 'Entomophagy': an evolving terminology in need of review. Journal of Insects as Food and Feed, 1(4), 293-305.

Tan, H.S.G., Fischer, A.R.H., Tinchan, P., Steenbekkers, L.P.A., Stieger, M., \& van Trijp, H.C.M. (2015). Insects as food: Exploring cultural exposure and individual experience as determinants of acceptance. Food Quality \& Preference 42, 78-89.

Bredie, W.L., Tan, H.S.G., \& Wendin, K. (2014). A comparative study on facially expressed emotions in response to basic tastes. Chemosensory Perception 7(1), 1-9.

Tan, H.S.G., Yu, B., Curran, P. \& Liu, S.-Q. (2011). Lipase-catalysed synthesis of natural aromaactive 2-phenylethyl esters in coconut cream. Food Chemistry 124, 80-84. 


\section{ABSTRACTS AND PRESENTATIONS}

Tan, H.S.G., Tinchan, P., Steenbekkers, L.P.A., \& Fischer, A.R.H. Psychological and product factors on the perception and evaluation of insects as food. $1^{\text {st }}$ Asian Sensory and Consumer Research Symposium, 11-13 May 2014, Singapore. Oral presentation.

Tan, H.S.G., Tinchan, P., Steenbekkers, L.P.A., Lakemond, C.M.M., \& Fischer, A.R.H. Sustainable critters or delicious fritters? Consumer perceptions of edible insects in the Netherlands and Thailand. $1^{\text {st }}$ Insects to Feed the World Conference, 14-17 May 2014, Ede, The Netherlands. Oral presentation.

Tan, H.S.G., Tinchan, P., Steenbekkers, L.P.A., Stieger, M., \& Fischer, A.R.H. Delicious Fritters or Disgusting Critters? Psychological and product factors that influence consumer perceptions of insects. $6^{\text {th }}$ European Conference for Sensory and Consumer Research, 7-10 September 2014, Copenhagen, Denmark. Poster presentation. Winner of the European Sensory Science Society Student Award.

Tan, H.S.G., Fischer, A.R.H., van Trijp, H.C.M., \& Stieger, M. Can nasty foods be tasty? Food appropriateness and sensory-liking of unusual foods. $11^{\text {th }}$ Pangborn Sensory Science Symposium, 23-27 August 2015, Gothenburg, Sweden. Poster presentation. Winner of the Rick Bell Memorial Scholarship.

Tan, H.S.G., van den Berg, E., \& Stieger, M. Introducing novel and unusual foods: The interplay of food neophobia and sensory appeal. 11 ${ }^{\text {th }}$ Pangborn Sensory Science Symposium, 23-27 August 2015, Gothenburg, Sweden. Poster and oral teaser presentations.

Tan, H.S.G., van den Berg, E., \& Stieger, M. The influence of product and individual factors on the acceptability insects as food. $2^{\text {nd }}$ Asian Sensory and Consumer Research Symposium, 15-17 May 2016, Shanghai, China. Poster presentation.

Tan, H.S.G., Verbaan, Y.T.., \& Stieger, M. Effects of product and sensory expectations on the liking and willingness to buy novel insect-based products. $2^{\text {nd }}$ Asian Sensory and Consumer Research Symposium, 15-17 May 2016, Shanghai, China. Poster presentation.

Tan, H.S.G., Fischer, A.R.H., van Trijp, H.C.M., \& Stieger, M. Eating insects: The challenges of introducing a novel protein source. Protein for Life Conference, 23-26 October, Ede, The Netherlands. Oral presentation. 


\section{OVERVIEW OF COMPLETED TRAINING ACTIVITIES}

\begin{tabular}{|c|c|c|}
\hline Discipline specific courses and activities & Organizer and location & Year \\
\hline PhD course in 'Industrial Food Proteins', & $\begin{array}{l}\text { Graduate school VLAG, } \\
\text { Wageningen, The Netherlands }\end{array}$ & 2013 \\
\hline $\begin{array}{l}\text { PhD course in 'Understanding and Measuring } \\
\text { Food Preferences' }\end{array}$ & $\begin{array}{l}\text { European Sensory Science Society, } \\
\text { Milan, Italy }\end{array}$ & 2013 \\
\hline $\begin{array}{l}\text { PhD course in 'Sensory Evaluation and Food } \\
\text { Preferences' }\end{array}$ & $\begin{array}{l}\text { University of Copenhagen, } \\
\text { Copenhagen, Denmark }\end{array}$ & 2014 \\
\hline $\begin{array}{l}1^{\text {st }} \text { Asian Sensory and Consumer Research } \\
\text { Symposium }\end{array}$ & $\begin{array}{l}\text { Elsevier, } \\
\text { Singapore, Singapore }\end{array}$ & 2014 \\
\hline $1^{\text {st }}$ Insects to Feed the World Conference & $\begin{array}{l}\text { Wageningen University \& FAO } \\
\text { Ede, The Netherlands }\end{array}$ & 2014 \\
\hline $\begin{array}{l}6^{\text {th }} \text { European Conference in Sensory and } \\
\text { Consumer Research }\end{array}$ & $\begin{array}{l}\text { Elsevier, } \\
\text { Copenhagen, Denmark }\end{array}$ & 2014 \\
\hline $11^{\text {th }}$ Pangborn Sensory Science Symposium & $\begin{array}{l}\text { Elsevier, } \\
\text { Gothenburg, Sweden }\end{array}$ & 2015 \\
\hline $\begin{array}{l}2^{\text {nd }} \text { Asian Sensory and Consumer Research } \\
\text { Symposium }\end{array}$ & $\begin{array}{l}\text { Elsevier, } \\
\text { Shanghai, China }\end{array}$ & 2016 \\
\hline $1^{\text {st }}$ Protein for Life Conference & $\begin{array}{l}\text { Wageningen University } \\
\text { Ede, The Netherlands }\end{array}$ & 2016 \\
\hline General Courses and Activities & Organizer and location & Year \\
\hline $\begin{array}{l}\text { Workshop on 'How to give and receive } \\
\text { feedback' }\end{array}$ & $\begin{array}{l}\text { Young AFSG, } \\
\text { Wageningen, The Netherlands }\end{array}$ & 2012 \\
\hline PhD Introduction Week & $\begin{array}{l}\text { Graduate school VLAG, } \\
\text { Baarlo, The Netherlands }\end{array}$ & 2013 \\
\hline Competence Assessment & & 2012 \\
\hline Course on 'Data Management' & & 2014 \\
\hline Course on 'Information Literacy and Endnote' & & 2014 \\
\hline $\begin{array}{l}\text { Course on 'Interpersonal Communication for } \\
\text { PhD students' }\end{array}$ & $\begin{array}{l}\text { Wageningen Graduate School, } \\
\text { Wageningen, The Netherlands }\end{array}$ & 2014 \\
\hline Course on 'Scientific Publishing' & & 2014 \\
\hline $\begin{array}{l}\text { Course on 'Techniques for writing and } \\
\text { presenting a scientific paper' }\end{array}$ & & 2014 \\
\hline $\begin{array}{l}\text { Course on 'Systematic Approaches to } \\
\text { Reviewing Literature' }\end{array}$ & & 2015 \\
\hline
\end{tabular}




\begin{tabular}{llc}
\hline Optional Courses and Activities & Organizer and location & Year \\
\hline $\begin{array}{l}\text { Masters course in 'Consumer Behaviour: } \\
\text { Concepts and Research Methods' }\end{array}$ & $\begin{array}{l}\text { Marketing and Consumer Behaviour, } \\
\text { Wageningen, The Netherlands }\end{array}$ & 2013 \\
Participating in the PhD study tour & $\begin{array}{l}\text { Food Quality and Design, } \\
\text { Singapore and Thailand }\end{array}$ & 2014 \\
Organisation of the PhD study tour & Food Quality and Design, \\
& Wageningen, The Netherlands & $2013-$ \\
Participation and presentation in the & Wageningen PhD Council & 2014 \\
$1^{\text {st } \text { Wageningen PhD Symposium }}$ & Wageningen, The Netherlands & 2015 \\
$22^{\text {nd }}$ European Nutrition Leadership Platform & European Nutrition Leadership Platform, & \\
Essentials Programme & Luxembourg & 2016 \\
& Marketing and Consumer Behaviour, & $2012-$ \\
Participation in PhD and staff meetings & Wageningen, The Netherlands & 2016 \\
& Food Quality and Design, & $2012-$ \\
& Wageningen, The Netherlands & 2016 \\
\hline
\end{tabular}


The research described in this thesis was part of the IP/OP Customized Nutrition programme, and in collaboration with Ynsect and VENIK (Branch Organisation of Dutch Insect Growers).

Financial support from Wageningen University and M. Ruig en Zonen B.V. for conducting the research and printing this thesis are gratefully acknowledged.

Cover design: Barend Tempelman and Hui Shan Grace Tan

Layout: Anika Oppermann and Hui Shan Grace Tan

Printed by: Gildeprint, Enschede, The Netherlands 